\title{
Statistical Modeling and Characterization of Induced Seismicity within the Western Canada Sedimentary Basin
}

Sid Kothari, The University of Western Ontario

Supervisor: Shcherbakov, Robert, The University of Western Ontario

A thesis submitted in partial fulfillment of the requirements for the Master of Science degree in Geophysics

(c) Sid Kothari 2019

Follow this and additional works at: https://ir.lib.uwo.ca/etd

Part of the Applied Statistics Commons, Geophysics and Seismology Commons, Statistical Models Commons, and the Tectonics and Structure Commons

\section{Recommended Citation}

Kothari, Sid, "Statistical Modeling and Characterization of Induced Seismicity within the Western Canada Sedimentary Basin" (2019). Electronic Thesis and Dissertation Repository. 6638.

https://ir.lib.uwo.ca/etd/6638

This Dissertation/Thesis is brought to you for free and open access by Scholarship@Western. It has been accepted for inclusion in Electronic Thesis and Dissertation Repository by an authorized administrator of Scholarship@Western. For more information, please contact wlswadmin@uwo.ca. 


\begin{abstract}
In western Canada, there has been an increase in seismic activity linked to anthropogenic energy-related operations including conventional hydrocarbon production, wastewater fluid injection and more recently hydraulic fracturing (HF). Statistical modeling and characterization of the space, time and magnitude distributions of the seismicity clusters is vital for a better understanding of induced earthquake processes and development of predictive models. In this work, a statistical analysis of the seismicity in the Western Canada Sedimentary Basin was performed across past and present time periods by utilizing a compiled earthquake catalogue for Alberta and eastern British Columbia. Specifically, the frequency-magnitude statistics were analyzed using the Gutenberg-Richter (GR) relation. The clustering of seismicity was studied using the Nearest-Neighbour Distance (NND) method and the Epidemic Type Aftershock Sequence (ETAS) model. The obtained results suggest that recent regional changes in the NND distributions, namely a disproportionate increase in loosely and tightly clustered seismic activity over time, are unnatural and likely due to the rise in HF operations for the development of unconventional resources. It is concluded that both these loosely and tightly clustered earthquake subpopulations differ measurably from what may be the region's tectonic seismic activity. Additionally, HF treatments have a greater probability of triggering swarm-like sequences that sharply spike the seismicity rate and are characterized by steeper frequencymagnitude distributions. Conventional production and wastewater disposal operations largely trigger loosely clustered activity with more typical magnitude-occurrence rates.
\end{abstract}

\title{
Keywords
}

Induced seismicity, earthquake clustering, statistical analysis, computer modeling, Alberta. 


\section{Lay Summary}

In western Canada, there has been an increase in earthquake activity linked to industrial activities including fossil fuel extraction, wastewater disposal and more recently hydraulic fracturing $(\mathrm{HF})$. Statistical modeling of earthquake phenomena is important for the understanding of the specific mechanisms involved in triggering earthquakes. In this work, a statistical analysis of the recorded earthquakes in the Western Canada Sedimentary Basin was performed. The results of this study suggest that there are discrete statistical differences between natural, tectonic earthquakes and those triggered artificially by human activity. Additionally, HF operations appear capable of triggering swarm-like sequences that temporarily increase the earthquake rate and tend to occur at small-to-moderate magnitudes. Conventional fuel production and wastewater disposal operations largely trigger earthquakes that are loosely clustered together in space and time across a broader magnitude range. 


\section{Co-Authorship Statement}

Sid Kothari performed the analyses and wrote the contents of this thesis under the supervision of Dr. Robert Shcherbakov. Sid wrote the code for all statistical methods and models used with the exception of the ETAS model, which was adapted from code written by Dr. Shcherbakov. 


\section{Acknowledgments}

First, I would like to thank my supervisor, Dr. Robert Shcherbakov, for his endless patience, guidance and support. He allowed me the freedom to explore new ideas and approaches to this thesis. The end result would not have been possible without him.

Second, I would like to thank Dr. Gail Atkinson for her encouragement and inspiration. Her positive input during the early stages of my research gave me the confidence to return and complete my studies.

Third, I would like to extend my gratitude to Dr. Richard Secco, who revealed the joys of teaching to me. He continually gave students the best learning experience he possibly could and I admired that greatly.

Finally, I would like to thank my friends and family. Dealing with health issues is not easy and is often frustrating; I am eternally grateful for their support. My mother never let me forget that I had unfinished business at Western University, and that the work I did might actually be of some benefit. 


\section{Table of Contents}

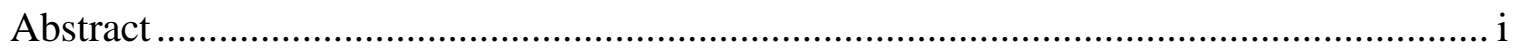

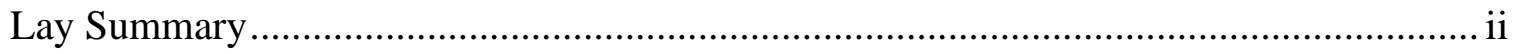

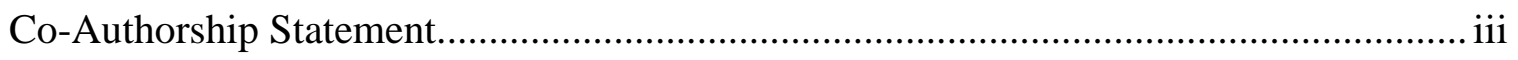

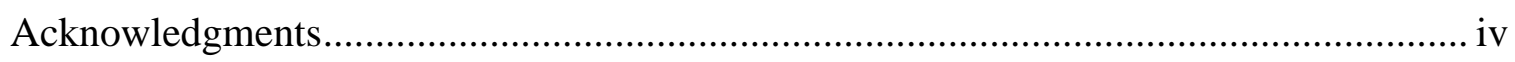

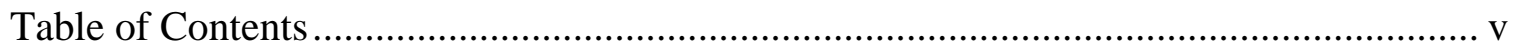

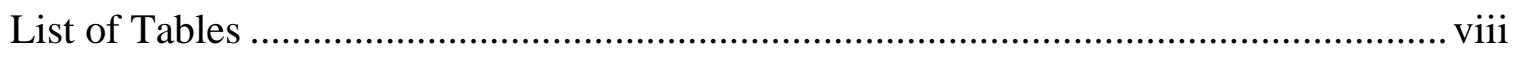

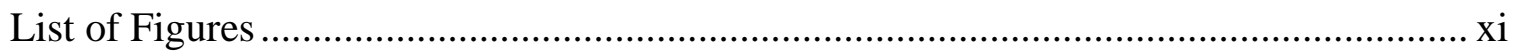

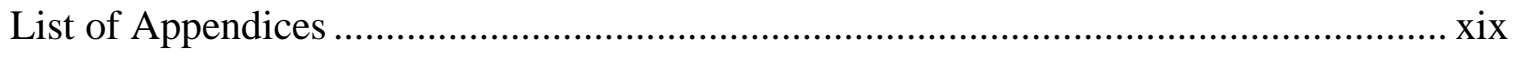

List of Abbreviations and Symbols....................................................................

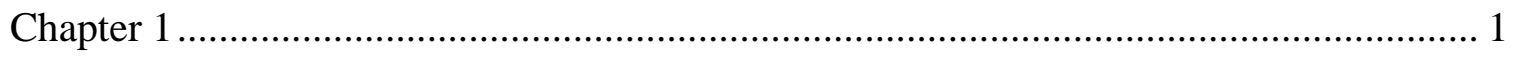

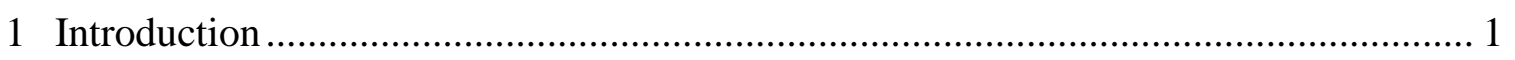

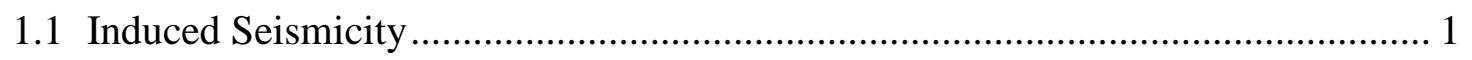

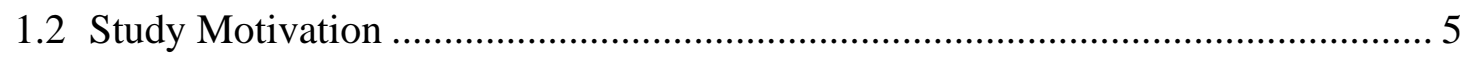

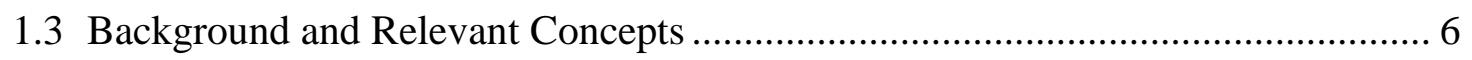

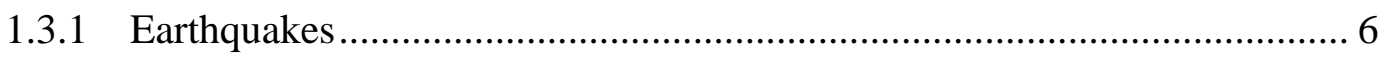

1.3.2 Earthquake Catalogues and the Gutenberg-Richter Relation ................... 7

1.3.3 Magnitude of Completeness ........................................................ 9

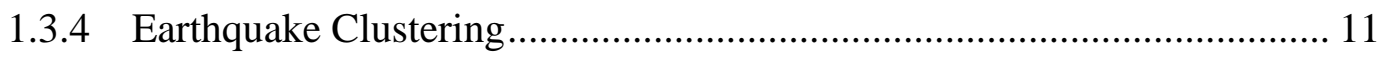

1.4 Thesis Structure and Summary of Results ................................................... 15

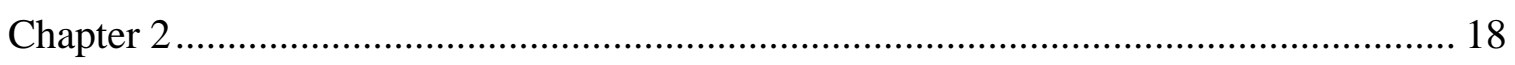

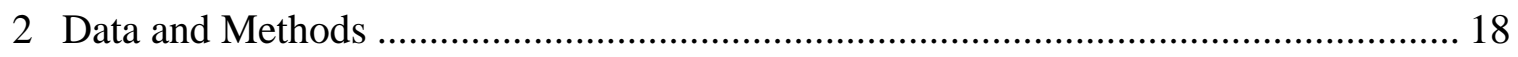

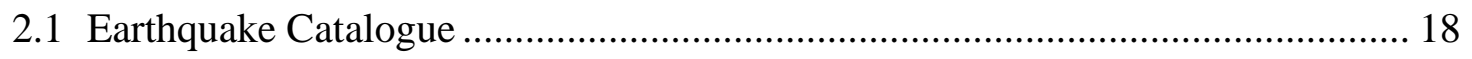

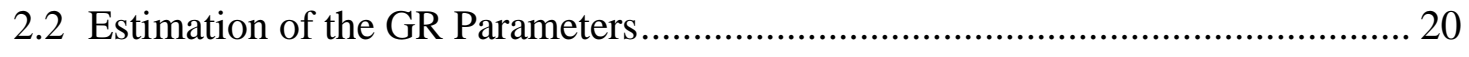

2.3 Catalogue-based Methods for the Estimation of Mc...................................... 22 
2.3.1 Method of Maximum Curvature (MAXC) …...................................... 22

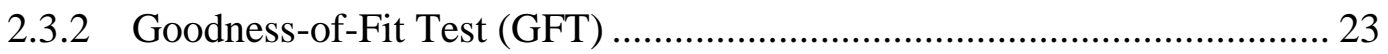

2.3.3 Method of $b$-value Stability (MBS) …................................................... 25

2.4 The Epidemic Type Aftershock Sequence (ETAS) Model................................ 27

2.5 The Nearest-Neighbour Distance (NND) Model ........................................... 30

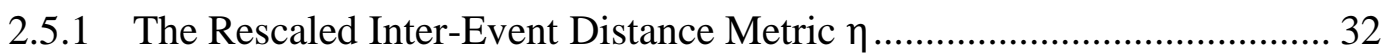

2.5.2 Formal Analysis of Modality in the $\eta$ Distribution ............................... 34

2.5.3 Classification of Event Clusters ..................................................... 37

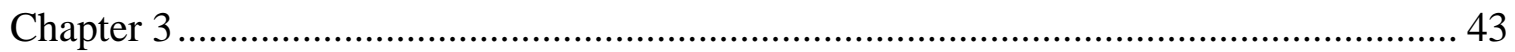

3 Regional Analysis of Nearest-Neighbour Distance Distributions within the Western Canada Sedimentary Basin ................................................................... 43

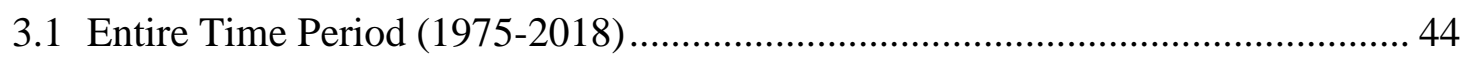

3.1.1 Comparative Analysis of Mixture Components .................................. 49

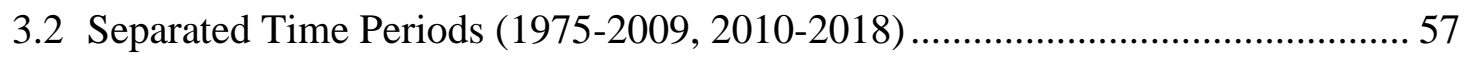

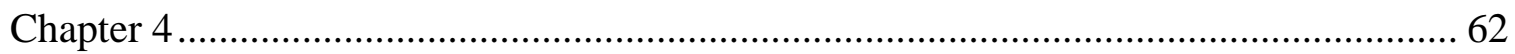

4 Case-by-case Investigation of Seismic Clustering within the Western Canada

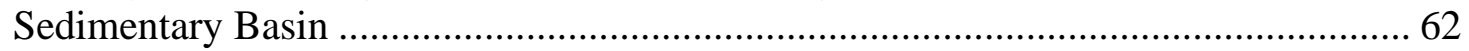

4.1 Rocky Mountain House Cluster (RMHC) - Conventional Gas Extraction

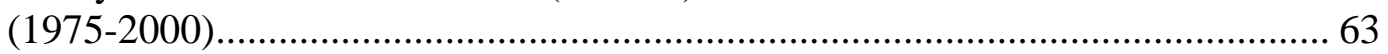

4.2 Montney Clusters (MC1 \& MC2) - Wastewater Disposal \& Hydraulic

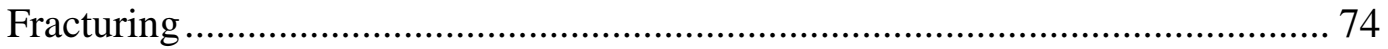

4.2.1 First Active Period (MC1) - Wastewater Disposal (1984-2009) ............. 75

4.2.2 Second Active Period (MC2) - Wastewater Disposal \& Hydraulic

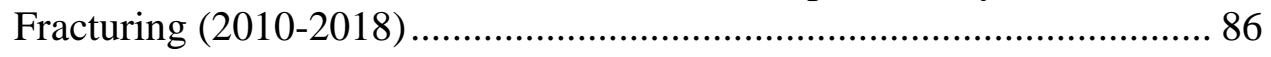

4.3 Fox Creek Cluster (FCC) - Hydraulic Fracturing (2013-2018) ......................... 98

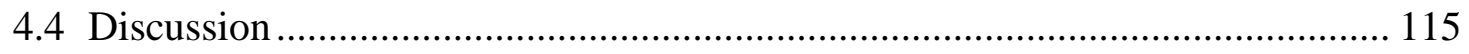

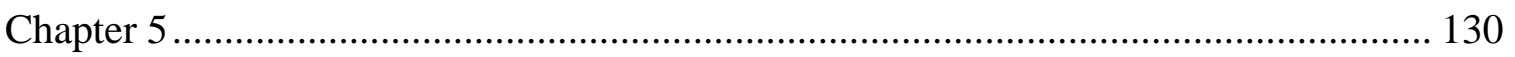

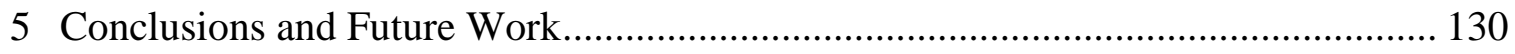




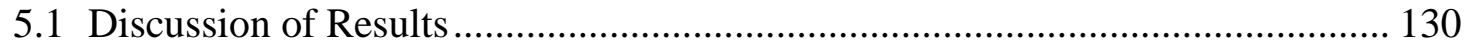

5.1.1 Regional Analysis of Seismicity within the WCSB (Chapter 3) ............ 130

5.1.2 Investigation of Seismic Clustering within the WCSB (Chapter 4) ....... 136

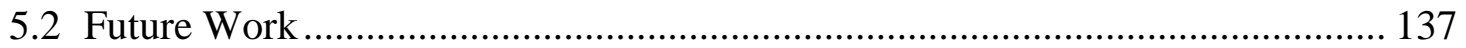

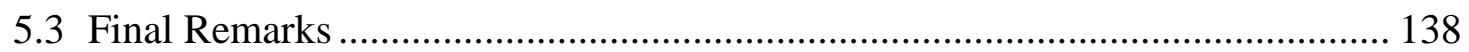

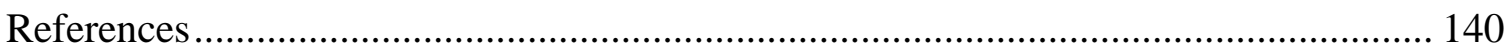

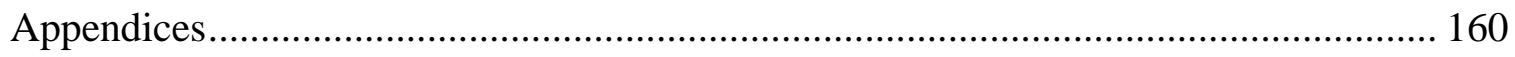

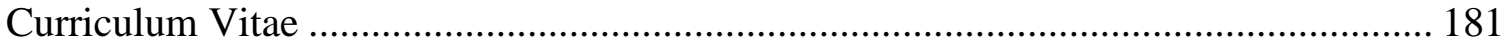




\section{List of Tables}

Table 3-1: Expectation Maximization results for the GMM applied to the regional WCSB catalogue (1975-2018) using M0 = 2.0. $k$ is the number of modes, AIC and BIC are the resulting Akaike and Bayes Information Criteria. Highlights show the minimized values.

Table 3-2: Gaussian mixture proportions for the regional WCSB catalogue (1975-2018) using M0 $=2.0$.

Table 3-3: EM results for the GMM applied to the separated time periods using M0 = 2.0. $k$ is the number of modes, AIC and BIC are the resulting Akaike and Bayes Information Criteria. Highlights show the minimized values

Table 3-4: NND Breakdown for the separated time periods using M0 $=2.0$. 61

Table 4-1: Summary of Mc and GR Analyses for the Rocky Mountain House cluster....66

Table 4-2: Event-Family Breakdown for the Rocky Mountain House cluster. .68

Table 4-3: Parameter sets for event families within the Rocky Mountain House cluster. N is the size of the sequence, $\mathrm{d}$ is the average leaf depth, $\delta$ is the normalized leaf depth, Bi is the inverted branching number, $\mathrm{A}$ is the spatial area, $\mathrm{td}$ is the decay period, and $\Delta \mathrm{M}$ is the magnitude differential.

Table 4-4: ETAS model parameters for the Rocky Mountain House cluster. $\mathrm{N}$ is the number of events modeled, $\mu$ is the constant background rate, $K$ is the aftershock productivity, $c$ is the temporal offset, $p$ is the exponential decay, $\alpha$ is the magnitudeaftershock dependence, and QOF is the quality of the model fit.

Table 4-5: Summary of Mc and GR Analyses for the Montney cluster 1. 78

Table 4-6: Event Family Breakdown for the Montney cluster 1. 80

Table 4-7: Parameter sets for event families within the Montney cluster $1 . \mathrm{N}$ is the size of the sequence, $\mathrm{d}$ is the average leaf depth, $\delta$ is the normalized leaf depth, Bi is the inverted 
branching number, $\mathrm{A}$ is the spatial area, $\mathrm{td}$ is the decay period, and $\Delta \mathrm{M}$ is the magnitude differential.

Table 4-8: ETAS model parameters for the Montney cluster $1 . \mathrm{N}$ is the number of events modeled, $\mu$ is the constant background rate, $K$ is the aftershock productivity, $c$ is the temporal offset, $p$ is the exponential decay, $\alpha$ is the magnitude-aftershock dependence, and QOF is the quality of the model fit.

Table 4-9: Summary of Mc and GR Analyses for the Montney cluster 2.

Table 4-10: Event Family Breakdown for the Montney cluster 2.

Table 4-11: Parameter sets for event families within the Montney cluster 2. $\mathrm{N}$ is the size of the sequence, $d$ is the average leaf depth, $\delta$ is the normalized leaf depth, Bi is the inverted branching number, $\mathrm{A}$ is the spatial area, td is the decay period, and $\Delta \mathrm{M}$ is the magnitude differential.

Table 4-12: ETAS model parameters for the Montney cluster 2. $\mathrm{N}$ is the number of events modeled, $\mu$ is the constant background rate, $K$ is the aftershock productivity, $c$ is the temporal offset, $p$ is the exponential decay, $\alpha$ is the magnitude-aftershock dependence, and QOF is the quality of the model fit. .98

Table 4-13: Summary of Mc and GR Analyses for the Fox Creek cluster. 101

Table 4-14: Event Family Breakdown for the Fox Creek cluster. 103

Table 4-15: Parameter sets for event families within the Fox Creek cluster. $\mathrm{N}$ is the size of the sequence, $\mathrm{d}$ is the average leaf depth, $\delta$ is the normalized leaf depth, Bi is the inverted branching number, $\mathrm{A}$ is the spatial area, td is the decay period, and $\Delta \mathrm{M}$ is the magnitude differential.

Table 4-16: ETAS model parameters for the Fox Creek cluster. $\mathrm{N}$ is the number of events modeled, $\mu$ is the constant background rate, $K$ is the aftershock productivity, $c$ is the temporal offset, $p$ is the exponential decay, $\alpha$ is the magnitude-aftershock dependence, and QOF is the quality of the model fit. 
Table 4-17: ETAS model parameters for the Fox Creek cluster using $\mu \approx 0$.

Table 4-18: Parameter sets for the largest 3 event families within the Rocky Mountain House cluster. $\mathrm{N}$ is the size of the sequence, $\mathrm{d}$ is the average leaf depth, $\delta$ is the normalized leaf depth, $\mathrm{Bi}$ is the inverted branching number, $\mathrm{A}$ is the spatial area, $t \mathrm{~d}$ is the decay period, and $\Delta \mathrm{M}$ is the magnitude differential. 121

Table 4-19: Parameter sets for the largest 3 event families within the Montney cluster 1. See Table 4-18 for parameter descriptions. 122

Table 4-20: Parameter sets for the largest 3 event families within the Montney cluster 2. See Table 4-18 for parameter descriptions.

Table 4-21: Parameter sets for the largest 3 event families within the Fox Creek cluster. See Table 4-18 for parameter descriptions.

Table 4-22: Mean and median parameter values for all significant event families within each investigated cluster. See Table 4-18 for parameter descriptions.

Table 4-23: ETAS model parameters for each investigated cluster. $\mathrm{N}$ is the number of events modeled, $\mu$ is the constant background rate, $K$ is the aftershock productivity, $c$ is the temporal offset, $p$ is the exponential decay, $\alpha$ is the magnitude-aftershock dependence, and QOF is the quality of the model fit. 


\section{List of Figures}

Figure 2-1: Map of $M \geq 2$ seismic events documented by the Composite Alberta Seismicity Catalogue (CASC) from 1975-2018. Marker size and colour indicate earthquake magnitude.

Figure 2-2: An example of the MAXC method applied to a sample frequency-magnitude distribution (FMD). Blue histogram represents the individual earthquake count per magnitude bin (non-cumulative FMD) and black dots represent the cumulative earthquake count per magnitude bin (cumulative FMD). Vertical dashed red line indicates the point of maximum curvature, which corresponds to the largest magnitude bin.

Figure 2-3: An example of the GFT method applied to a sample FMD. Pink squares represent the synthetic distribution that best describes the data. Grey squares represent the rejected synthetic distributions. Vertical dashed pink line indicates the corresponding GFT Mc.

Figure 2-4: An example of the MBS method applied to a sample catalogue. Blue solid line represents the MLE $b$-values for a range of cut-off magnitudes and dashed black lines are the uncertainty. Orange line with triangle markers represents the rolling average (bave). Vertical dashed yellow line indicates completeness as determined by Woessner \& Wiemer (2005) (where bave first dips below uncertainty), which is considered as the lower bound for Mc in Chapter 4. Vertical dashed purple line indicates where $\Delta b$ is minimized, which is considered as the upper bound.

Figure 2-5: Simplified description of the NND method workflow

Figure 2-6: NND distributions of southern California earthquakes for different cut-off magnitudes. $(\mathrm{a}, \mathrm{b})$ The joint distributions of the temporal $(T)$ and spatial $(R)$ components; diagonal line represents the separation between the two modes. (c, d) The normalized densities of $\eta$ values; modal separation occurs at log10ๆthresh $\approx-5$. (a, c) $M 0=2$; (b, d) $\mathrm{M} 0=3$. (From Zaliapin \& Ben-Zion, 2013a). 
Figure 2-7: Event classification methodology. Left-hand side: a single event (white circle), characterized by only weak links to both potential parent and offspring events. Right-hand side: an event family connected by strong links. The dark circle is the largest event and is labeled the mainshock; it is preceded in time by smaller foreshocks (white squares) and succeeded by smaller aftershocks (grey squares). (From Zaliapin \& BenZion, 2013a).

Figure 2-8: Cluster 1: characterization of an example "burst" sequence. Circles represent earthquakes and are coloured chronologically from darkest to lightest; grey lines represent the strong links connecting them. a) Event family magnitudes vs. times of occurrence in days. b) Spatial map of events; yellow border outlines the hull area occupied by the sequence. c) The topological family tree, which branches downward in time.

Figure 2-9: Cluster 2: characterization of an example "swarm" sequence. Circles represent earthquakes and are coloured chronologically from darkest to lightest; grey lines represent the strong links connecting them. a) Event family magnitudes vs. times of occurrence in days. b) Spatial map of events; yellow border outlines the hull area occupied by the sequence. c) The topological family tree, which branches downward in time.

Figure 3-1: Map of study region. Markers are $M \geq 2$ seismic events between 1975-2018; size and colour indicate earthquake magnitude.

Figure 3-2: NND distribution of the regional WCSB catalogue (1975-2018) using M0 = 2.0. a) Joint distribution of the temporal and spatial components $(T, R)$. Bold white line indicates the threshold $\log 10 \eta$ thresh between tightly clustered and loosely clustered components. Thin white line indicates the threshold log10ntect between loosely clustered and deep-background components. Colour bar indicates frequency of interevent distance occurrence. b) Normalized density of $\eta$ values. Solid black line is

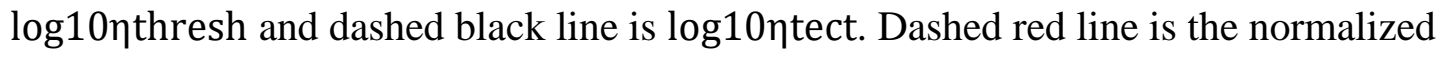
probability density function of the Gaussian mixture and black crosses are the component means. 
Figure 3-3: Individual histograms of inter-event time $(T)$ and space $(\mathrm{R})$ for the regional WCSB catalogue (1975-2018) using M0 = 2.0. a) Histogram of rescaled times $T$. b) Histogram of rescaled distances $R$.

Figure 3-4: Joint $(T, R)$ distributions of individual modes for the regional WCSB catalogue (1975-2018) using M0 = 2.0. Colour bar reflects relative NND intensity. Bold white line indicates the threshold log10nthresh between tightly clustered and loosely clustered components. Thin white line indicates the threshold log10 †tect between loosely clustered and deep-background components. a) Entire distribution. b) Tightly clustered mode. c) Loosely clustered mode. d) Deep-background

Figure 3-5: FMDs and estimated GR $b$-values of the regional WCSB catalogue (19752018) using $\mathrm{Mc}=3.0$. Coloured histograms represent the non-cumulative distribution and black dots represent the cumulative distributions. Dashed black line indicates the cutoff magnitude. Coloured lines represent the modeled GR relations. a) Entire catalogue. b) Tightly clustered mode. c) Loosely clustered mode. d) Deep-background. .52

Figure 3-6: Spatial map of earthquakes for the regional WCSB catalogue (1975-2018) using M0 $=2.0$, represented in terms of their nearest-neighbour distance categorization. Blue markers are the deep-background, orange markers are the loosely clustered events and yellow markers are the tightly clustered events. Marker size indicates magnitude. Dashed boxes surround areas of suspected induced clustering.

Figure 3-7: Cumulative number of detected event families vs. the $\eta$ values of their ancestor event (blue line). Dashed black line represents $\log 10 \eta$ tect $\approx-1.7$, the separation point between the two background modes. a) Non-single event families ( $\mathrm{N} \geq$ 2). b) Significant event families $(\mathrm{N} \geq 5)$.

Figure 3-8: Comparison of NND distributions of the regional WCSB catalogue across time using M0 = 2.0. (a, b) Joint distributions of the temporal and spatial components $(T$, $R$ ). Bold white line indicates the threshold log10ๆthresh between tightly clustered and loosely clustered components. Thin white line indicates the threshold log10ntect between loosely clustered and deep-background components. Colour bar indicates frequency of occurrence. (c, d) Normalized densities of $\boldsymbol{\eta}$ values. Solid black line is 
$\log 10 \eta$ thresh and dashed black line is $\log 10 \eta$ tect. Dashed red line is the normalized probability density function of the Gaussian mixture and black crosses are the component means. (a, c) 1975-2009, both background modes are dominant. (b, d) 2010-2018, deepbackground shrinks while tightly clustered mode appears. The loosely clustered subpopulation is common to both time frames.

Figure 4-1: Map of the Rocky Mountain House cluster study area between 1975-2000. Dashed circle represents a $20 \mathrm{~km}$ radius from the coordinates [-115.24, 52.17]. Markers are seismic events. Coloured markers are the data points used for analysis.

Figure 4-2: Mc and GR Analyses for the Rocky Mountain House cluster. a) Maximum Curvature Method (MAXC). b) Goodness-of-Fit Test (GFT). c) Method of $b$-value Stability (MBS). d) GR parameter estimation using determined $\mathrm{M}_{\mathrm{c}}$

Figure 4-3: Nearest-Neighbour Distance distribution for the Rocky Mountain House cluster. a) Joint distribution of the temporal and spatial components $(T, R)$. b) Normalized density of $\eta$ values.

Figure 4-4: Magnitude vs. time evolution of event families within the Rocky Mountain House cluster. Lower plot: Event magnitudes vs. time. Upper plot: Event-type classification over time. White squares are singles, red circles are foreshocks, red triangles are mainshocks, blue circles are aftershocks, and green circles are aftershocksof-aftershocks. Grey lines represent the strong links connecting individual event families.

Figure 4-5: Event Family Structures in the Rocky Mountain House cluster. a) Moment magnitude vs. Time in days. b) Spatial map. Yellow border outlines the hull area occupied by the sequence. c) Directed tree graph in dimensionless space. Data points are coloured chronologically from darkest to lightest.

Figure 4-6: ETAS Model of the Rocky Mountain House cluster using Mc $=2.3$. a) Original time plot. Dashed orange line is the observed cumulative rate of seismicity. Solid line is the optimized model. b) Transformed time plot. Solid line represents the "perfect" model fit. 
Figure 4-7: Map of the Montney cluster 1 study area between 1984-2009. Dashed circle represents a $75 \mathrm{~km}$ radius from the coordinates [-121.6, 56.5]. Markers are seismic events. Coloured markers are the data points used for analysis.

Figure 4-8: Mc and GR Analyses for the Montney cluster 1. a) Maximum Curvature Method (MAXC). b) Goodness-of-Fit Test (GFT). c) Method of $b$-value Stability (MBS). d) GR parameter estimation using determined Mc.

Figure 4-9: Nearest-Neighbour Distance distribution for the Montney cluster 1. a) Joint distribution of the temporal and spatial components $(T, R)$. b) Normalized density of $\eta$ values.

Figure 4-10: Magnitude-Time evolution of event families of the Montney cluster 1. Lower plot: Event magnitudes vs. time. Upper plot: Event-type classification over time. White squares are singles, red circles are foreshocks, red triangles are mainshocks, blue circles are aftershocks, and green circles are aftershocks-of-aftershocks. Grey lines represent the strong links connecting individual event families

Figure 4-11: Event Family Structures within the Montney cluster 1. a) Moment magnitude vs. Time in days. b) Spatial map. Yellow border outlines the hull area occupied by the sequence. c) Directed tree graph in dimensionless space. Data points are coloured chronologically from darkest to lightest.

Figure 4-12: ETAS Model of the Montney cluster 1 using Mc = 2.7. a) Original time plot. Dashed orange line is the observed cumulative rate of seismicity. Solid line is the optimized model. b) Transformed time plot. Solid line represents the "perfect" model fit.

Figure 4-13: Map of the Montney cluster 2 study area between 2010-2018. Dashed circle represents a $150 \mathrm{~km}$ radius from the coordinates [-121.6, 56.5]. Markers are seismic events. Coloured markers are the data points used for analysis.

Figure 4-14: Mc and GR Analyses for the Montney cluster 2. a) Maximum Curvature Method (MAXC). b) Goodness-of-Fit Test (GFT). c) Method of $b$-value Stability (MBS). d) GR parameter estimation using determined Mc. .89 
Figure 4-15: Nearest-Neighbour Distance distribution for the Montney cluster 2. a) Joint distribution of the temporal and spatial components $(T, R)$. b) Normalized density of $\eta$ values.

Figure 4-16: Magnitude-Time evolution of event families of the Montney cluster 2. Lower plot: Event magnitudes vs. time. Upper plot: Event-type classification over time. White squares are singles, red circles are foreshocks, red triangles are mainshocks, blue circles are aftershocks, and green circles are aftershocks-of-aftershocks. Grey lines represent the strong links connecting individual event families 92

Figure 4-17: Event Family Structures within the Montney cluster 2. a) Moment magnitude vs. Time in days. b) Spatial map. Yellow border outlines the hull area occupied by the sequence. c) Directed tree graph in dimensionless space. Data points are coloured chronologically from darkest to lightest. 95

Figure 4-18: ETAS Model of the Montney cluster 2. a) Original time plot. Dashed orange line is the observed cumulative rate of seismicity. Solid line is the optimized model. b) Transformed time plot. Solid line represents the "perfect" model fit

Figure 4-19: Map of the Fox Creek cluster study area between 2013-2018. Dashed circle represents a $45 \mathrm{~km}$ radius from the coordinates [-117.4, 54.4]. Markers are seismic events. Coloured markers are the data points used for analysis.

Figure 4-20: Mc and GR Analyses for the Fox Creek cluster. a) Maximum Curvature Method (MAXC). b) Goodness-of-Fit Test (GFT). c) Method of $b$-value Stability (MBS). d) GR parameter estimation using determined Mc.

Figure 4-21: Nearest-Neighbour Distance distribution for the Fox Creek cluster. a) Joint distribution of the temporal and spatial components $(T, R)$. b) Normalized density of $\eta$ values. 103

Figure 4-22: Magnitude-Time evolution of event families within the Fox Creek cluster. Lower plot: Event magnitudes vs. time. Upper plot: Event-type classification over time. White squares are singles, red circles are foreshocks, red triangles are mainshocks, blue 
circles are aftershocks, and green circles are aftershocks-of-aftershocks. Grey lines represent the strong links connecting individual event families.

Figure 4-23: Event Family Structures for the Fox Creek cluster. a) Moment magnitude vs. Time in days. b) Spatial map. Yellow border outlines the hull area occupied by the sequence. c) Directed tree graph in dimensionless space. Data points are coloured chronologically from darkest to lightest. 109

Figure 4-24: Event Sequence Map for the Fox Creek cluster. Coloured markers represent event-family members. Coloured shapes mark the spatial boundaries of each family...111

Figure 4-25: ETAS Model of the Fox Creek cluster using Mc $=2.5$. a) Original time plot. Dashed orange line is the observed cumulative rate of seismicity. Solid line is the optimized model. b) Transformed time plot. Solid line represents the "perfect" model fit.

Figure 4-26: ETAS Model of the Fox Creek cluster using Mc $=2.5$ and $\mu \approx 0$. a) Original time plot. Dashed orange line is the observed cumulative rate of seismicity. Solid line is the optimized model. b) Transformed time plot. Solid line represents the "perfect" model fit.

Figure 4-27: Frequency-Magnitude Distributions and estimated GR parameters for each investigated cluster. Coloured histograms represent the non-cumulative magnitude distributions and black dots represent the cumulative magnitude distributions. Dashed black lines indicate the determined local magnitude of completeness. Coloured lines represent the modeled GR relations. a) Rocky Mountain House cluster. b) Montney cluster 1. c) Montney cluster 2. d) Fox Creek cluster.

Figure 4-28: Joint NND distributions of the temporal and spatial inter-event distances ( $T$, $R$ ) for each investigated cluster. Bold white line indicates the threshold log10ๆthresh between tightly and loosely clustered components. Thin white line indicates the threshold $\log 10 \eta$ tect between the loosely clustered and deep-background. Colour bar reflects inter-event distance occurrence probability. a) Rocky Mountain House cluster. b) Montney cluster 1. c) Montney cluster 2. d) Fox Creek cluster. 
Figure 4-29: Largest 3 NND Event Families identified within the Rocky Mountain House cluster. a) Moment magnitude vs. Time in days. b) Spatial map. Yellow border outlines the hull area occupied by the sequence. c) Directed tree graph in dimensionless space. Data points are coloured chronologically from darkest to lightest.

Figure 4-30: Largest 3 NND Event Families identified within the Montney cluster 1. See Figure 4-29 for a graphical description.

Figure 4-31: Largest 3 NND Event Families identified within the Montney cluster 2. See Figure 4-29 for a graphical description.

Figure 4-32: Largest 3 NND Event Families identified within the Fox Creek cluster. See Figure 4-29 for a graphical description.

Figure 4-33: ETAS Models in original time for each investigated cluster. Dashed orange lines are the observed cumulative rates of seismicity. Solid coloured lines are the optimized models. a) Rocky Mountain House cluster. b) Montney cluster 1. c) Montney cluster 2. d) Fox Creek cluster.

Figure 5-1: Seismicity surrounding the Coso Geothermal Field from 1981-2013. Colour corresponds to time with younger seismicity projected on top of older seismicity. Wellbore trajectories are shown as black lines. From Schoenball et al. (2015).

Figure 5-2: Joint (T, R) histograms for the Coso Geothermal Field and surrounding area. Study areas (top row) A-B and (bottom row) B, for different time periods of the same length. The first time interval (left-hand side) is mostly pre-production, whereas the later intervals are co-production. The colour scale is normalized for each plot. From Schoenball et al. (2015). 133

Figure 5-3: Joint (T, R) distributions for Oklahoma across different time periods. a) Entire catalogue (1970-2016). b) Pre injection-related seismicity (1970-2009). (c, d) Injection-related seismicity (2010-2013, 2014-2016). Ellipses highlight the pre injectionrelated background. Diagonal lines represent constant $\log 10 \eta$ values and horizontal lines represent constant log10R values. From Vasylkivska \& Huerta (2017). 


\section{List of Appendices}

Appendix A: Nearest-Neighbour Distance (NND) distributions and corresponding Gaussian mixture model (GMM) results of the regional WCSB catalogue (1975-2018)

for different cut-off magnitudes..................................................................... 160

Appendix B: Nearest-Neighbour Distance (NND) distributions and corresponding Gaussian mixture model (GMM) results of the regional WCSB catalogue for separated time periods (1975-2009 and 2010-2018) using different cut-off magnitudes.............. 170 


\section{List of Abbreviations and Symbols}

AIC

BIC

B. C.

CASC

ETAS

EM

FCC

FMD

GMM

GFT

GR

HF

$M_{0}$

$M_{c}$

MBS

MAXC

$\mathrm{M}_{\mathrm{w}}, \mathbf{M}$, or $\mathrm{M}$

MC

NND
Akaike information criterion

Bayesian information criterion

British Columbia

Composite Alberta Seismicity Catalogue

Epidemic Type Aftershock Sequence (model)

Expectation Maximization

Fox Creek cluster

Frequency-magnitude distribution

Gaussian mixture model

Goodness-of-fit test

Gutenberg-Richter (relation/law/model)

Hydraulic fracturing

Magnitude cut-off

Magnitude of completeness

Method of $b$-value stability

Method of maximum curvature

Moment magnitude

Montney cluster $(1 \& 2)$

Nearest-Neighbor Distance (model) 
QOF

RMHC

WCSB
Quality of fit

Rocky Mountain House cluster

Western Canada Sedimentary Basin 


\section{Chapter 1}

\section{Introduction}

\subsection{Induced Seismicity}

Human activities, such as hydrocarbon production, reservoir impoundment, mining and geothermal energy extraction, can alter subsurface stress regimes through the withdrawal or injection of fluid, reservoir compaction, excess surface loading, ground subsidence and so on (Grigoli et al., 2017; Doglioni, 2018; Keranen \& Weingarten, 2018). In some cases these stress perturbations result in earthquakes, particularly in areas characterized by higher states of stress and/or preexisting, well-oriented faults. The involvement of propagating fluid further complicates matters; it is capable of destabilizing fault structures by altering pore pressure and reducing effective stress (Hainzl, 2004; Holland, 2013; Ogwari \& Horton, 2016) as well as deforming rock through the transfer of poroelastic stress (Segall et al., 1995; Wang \& Kümpel, 2003; Hamiel et al., 2004; Ben-Zion \& Lyakhovsky, 2006; Segall \& Lu, 2015; Chang \& Segall, 2016).

In the United States, specifically the mid-west including Oklahoma, Arkansas, and Texas, numerous earthquakes and earthquake sequences have been attributed to wastewater disposal wells operating in close proximity (Ellsworth, 2013; Llenos \& Michael, 2013; van der Elst et al., 2013; Keranen et al., 2014; Hornbach et al., 2016; Schoenball \& Ellsworth, 2017). These wells inject large quantities of excess flow-back fluid from associated oil and gas production operations deep into underground reservoirs in large volumes and at high pressures. This fluid can travel downward into the crystalline basement and interact with deeper fault structures, where most of the associated seismic events occur (Horton, 2012; Ellsworth, 2013; Keranen et al., 2014; McClure et al., 2017; Shah \& Keller, 2017). In 2016 alone, Oklahoma observed 3 $\boldsymbol{M} \geq 5$ earthquakes, including an M5.8 event in Pawnee that was the largest event recorded in the state's history. All 3 events occurred within $10 \mathrm{~km}$ of wastewater disposal wells and appeared to have seismic moment (energy) releases that scaled with the net volume of nearfield injection (McGarr \& Barbour, 2017). It is possible that geologic conditions allowed for the channeling of fluid and pressure front from more remote operations as well. 
In western Canada, there has been a notable rise in the rate of earthquakes coinciding with the implementation of unconventional extraction technology developed for the production of oil and gas, known as hydraulic fracturing (HF) or "fracking" (B.C. Oil and Gas Commission, 2012, 2014; Schultz et al., 2015c; Bao \& Eaton, 2016; Deng et al., 2016; Ghofrani \& Atkinson, 2016; Schultz et al., 2016; Wang et al., 2016; Schultz et al., 2017; Eaton et al., 2018; Schultz et al., 2018; Zhang et al., 2019). Fractures are created or enhanced within a target formation holding desired hydrocarbons, typically tight (low-permeability) sedimentary layers, such as shale, by the pumping of chemical slurry into segments of the rock over several stages. Polyacrylamide or a similar viscosity-reducing chemical is added to the fluid so that it can be pumped at a very high rate, up to 100 barrels per minute. A fine-grained "proppant" in the mixture, often sand, remains in these fractures to allow for the flow-back of targeted fossil fuels into the wellbore (Hyder \& Lerner, 2014; Smith \& Montgomery, 2015). Wells are increasingly drilled at a deviated or horizontal angle as they approach a reservoir, in order to engage a much greater portion of the source rock than would have been reached vertically. This approach has prompted a dramatic growth in the number of possible fracture stages per wellbore and consequently increased the average total volume of high-pressure fluid injected; it is common for 40 or more treatments to be applied presently, compared to the 5-10 stages used less than a decade ago or the 1-4 stages standard to vertical wells (King, 2010). These new technologies have greatly improved the potential for fossil fuel recovery and allowed for previously unrecoverable resources to be exploited. However, the associated rise in induced seismicity appears highly concentrated near some HF operations and cannot be fully accounted for by the deployment of denser seismic monitoring networks and more sensitive instruments (Schultz et al., 2015b; Atkinson et al., 2016; Cui \& Atkinson, 2016).

While the fundamental mechanisms for wastewater disposal-related seismicity are generally agreed upon, namely the diffusion of pore pressure promoting shear-failure along preexisting faults, the triggering processes of HF-induced seismicity remain less well understood. Fault reactivation may be attributable to several different sources, including hydraulic transmission between existing faults, new fractures and fluid injection wells (Galloway et al., 2018; Skoumal et al., 2018), fluid diffusion through semi-permeable rock causing pore pressure changes (Bao \& Eaton, 2016), post-injection stress relaxation (Hajati et al., 2015), fluid-induced aseismic slip (Scuderi \& Collettini, 2016; Scuderi et al., 2017; Bhattacharya \& Viesca, 2019), fault maturity 
(Kozłowska et al., 2018; Brudzinski \& Kozłowska, 2019), tectonic strain rates (Kao et al., 2018), and intra-cluster elastic stress redistribution and triggering of micro-seismicity (Stein, 1999; Sagiya et al., 2002; Maghsoudi et al., 2016, 2018). On the other hand, a recent study by Kettlety et al. (2019) showed that elastic stress transfer may weakly promote failure initially during HF and later actually inhibit further slip. The unequal distribution of the recent clustered seismicity in western Canada coupled with its high temporal and spatial correlation with a low number of individual wells and well pads (Schultz et al., 2015c; Atkinson et al., 2016; Bao \& Eaton, 2016; Schultz et al., 2017; Galloway et al., 2018) imply that local geology also plays a significant role in the nucleation of induced earthquakes. Similar compositions and volumes of injected fluids can result in widely ranging levels of seismic productivity depending in part on the geologic conditions where they are used, as has been observed in the United States (e.g. Hornbach et al., 2016; Keranen \& Weingarten, 2018) and in Canada (Schultz et al., 2016; Pawley et al., 2018). It is evident that the hazard related to anthropogenic seismicity will remain a pressing concern in the future, as technology continues to develop in the unconventional energy sectors and new, previously inaccessible resources are targeted. This has spurred discussion in both the public and scientific communities, and has increased the need for a deeper understanding and more thorough characterization of induced seismicity. As the underlying processes and geological settings of individual cases are complex and can differ significantly, statistical modeling of observed earthquakes can be a useful method to approximate system dynamics and help predict future behavior (Ogata, 1988, 1989; Baiesi \& Paczuski, 2004; Ghofrani \& Atkinson, 2016; Zaliapin \& Ben-Zion, 2016; McClure et al., 2017; Igonin et al., 2018).

This study focuses on Alberta and eastern British Columbia where seismicity has been low historically, with the majority of tectonic events occurring along the foreland belt of the Rocky Mountain range (Rogers \& Horner, 1991). However, since the onset of hydrocarbon extraction and associated wastewater disposal, first in the Duvernay and Cardium formations in lower central Alberta near the town of Rocky Mountain House and subsequently other, less permeable reservoirs to the north and south including within the Horn River Basin and near the towns of Fort Nelson, Fox Creek, Fort St. John and Cardston, there has been a rise in spatiotemporally clustered seismicity within the region. Atkinson et al. (2016) estimated that approximately 0.2$0.4 \%$ of HF wells and $\sim 1 \%$ of disposal wells operating within the Western Canada Sedimentary 
Basin (WCSB) have directly contributed to the occurrence of earthquakes $\boldsymbol{M} \geq 3$ between 1985-2015. However, the clusters that have arisen occur in previously quiescent areas and appear to be spatiotemporally linked to nearby human activity.

Baranova et al. (1999) linked the clustering near Rocky Mountain House to a depletion of pore pressure and an accumulation of compaction-related stress beneath the nearby Strachan gas extraction field between the 1970s and 1990s. A strong correlation between extraction rates and earthquake productivity was confirmed by Eaton \& Mahani (2015), who agreed that the seismic activity lagged commencement of production by approximately five years, and later regressed to quiescence as operations declined by the new millennium. Several studies have provided evidence of extended HF-related fluid injection driving the recent seismicity via pore pressure increase and poroelastic stress perturbation west of Fox Creek, beginning in December 2013 as a series of clusters near Crooked Lake and continuing intermittently to the present. Bao \& Eaton (2016) demonstrated that the rate of seismic clustering spiked markedly during certain proximate HF treatments and subsequently decreased post-injection. Further, this immediate stress response appeared to be followed by earthquake re-nucleation several weeks after completion, indicating that fluid pressurization may also perturb faults over longer periods of time. Similar conclusions were reached by Schultz et al. (2015c, 2017) who used crosscorrelation detection methods to augment their seismic dataset and scope of analysis. In both studies the authors found that induced earthquakes tended to group closely together in space and time, which was interpreted as repeated stimulation of the same fault structure through pore pressure perturbation within the low-permeability source rock. The events chiefly occurred during or immediately after specific nearby HF treatments and could be separated into distinct sequences. Some of these sequences emerged as two stages, with the second stage spatially distributed further from the suspicious wells (up to $\sim 5 \mathrm{~km}$ ), lasting months after shut-in and consisting of fewer but occasionally larger events. It is not well understood why only certain wells appear seismogenic among the thousands operating within the Duvernay formation, but recent studies by Schultz et al. (2016) and Pawley et al. (2018) suggest that geological susceptibility to factors, such as higher levels of lithium concentration, proximity to fossil reef margins and basement faults, and the natural seismicity rate, may contribute to triggering induced earthquakes. Induced seismicity has also been identified within the Montney formation and the Horn River Basin in northeastern British Columbia. In the 1980s and 1990s, fluid 
injection for secondary oil recovery and wastewater disposal had most likely induced earthquake clustering northwest of Fort St. John (Horner et al., 1994). Since the mid-to-late 2000s, significant HF and wastewater injection has taken place both within the Montney and the Horn River Basin's Muskwa and Evie shale formations. The B.C. Oil and Gas Commission $(2012,2014)$ conducted individual reports on these occurrences and attributed the majority of detected seismicity to human activity, based on spatiotemporal links to well activity and pumping rates as well as identification of nearby subsurface faults using 3-D seismic mapping provided by the operators.

\subsection{Study Motivation}

While many other studied regions tend to have a single dominant mechanism driving the induced earthquake activity, such as large-scale wastewater disposal in the central United States (Horton, 2012; Ellsworth, 2013; Llenos \& Michael, 2013; van der Elst et al., 2013) or the geothermal energy operations in southern California (Brodsky \& Lajoie, 2013; Zaliapin \& BenZion, 2013a), the WCSB is unique with its multiplicity of local triggering mechanisms. Additionally, the low natural occurrence rate, which has allowed for a relatively straightforward identification of the recent surge in anthropogenic seismicity (Atkinson et al., 2016) compared to other regions (e.g. Schoenball et al., 2015), potentially increases the seismic hazard of the induced activity. These factors offer significant motivation to analyze the regional changes in earthquake space, time, and magnitude distributions. Moreover, it is worthwhile to compare the different clusters and their triggers statistically, particularly cases of conventional production and disposal versus the current hydraulic fracturing-related seismicity. In this thesis, I aim to characterize the regional and clustered earthquakes using three statistical models: the Gutenberg-Richter (GR) relation, the Nearest-Neighbour Distance (NND) method and the Epidemic Type Aftershock Sequence (ETAS) model (described in detail below). The main objectives are twofold; the first is to demonstrate the fundamental statistical differences in the regional seismicity distributions over time and the second is to illuminate specific features of the induced clustering that could be expected or recognized when performing certain types of operations, particularly hydraulic fracturing. The results of this study may help in the identification of induced seismic activity in areas where discrimination is more difficult, as well 
as aid in hazard assessment and mitigation efforts by increasing understanding of case-specific earthquake triggering processes.

\subsection{Background and Relevant Concepts}

\subsubsection{Earthquakes}

During an earthquake, stored seismic energy is released due to the sudden movement of tectonic plates along oriented surfaces of separation known as faults. The majority of global earthquakes are concentrated near plate boundaries, where rocks are subject to severe deformation from the accumulated stress of opposing plate movements. Part of this progressive loading is redistributed inelastically through the interiors, resulting in areas of intra-plate stress accretion and conditions for internal fault rupture. If the restrictive forces that clamp existing asperities together (namely the frictional force and effective stress) are overcome, faults will fail and displacement will occur until sufficient energy is released and the stress balance is restored (Kanamori \& Brodsky, 2001; Scholz, 2002; Abercrombie, 2006; Segall, 2010; Udias et al., 2013; Moczo et al., 2014). As mentioned above, external factors, such as natural or artificial fluid intrusion, can expedite failure processes considerably by decreasing slip resistance through poroelastic stress perturbations and pore pressure changes (Husen et al., 2004; van Stiphout et al., 2009; Bachmann et al., 2012; Chang \& Segall, 2016; Scuderi \& Collettini, 2016; Galloway et al., 2018).

The accumulated strain energy of an earthquake is partially discharged as a shock or pulse, which is propagated as seismic waves in an amount proportional to the surface area of the rupture and the length of slip (Scholz, 2002). The size of an earthquake is normally estimated from the amplitude, frequency and/or duration of ground motions at an approximated distance from the source, detected using instruments called seismometers and recorded at seismic stations. Various scales to quantify relative earthquake sizes have been introduced in the literature, including the local (Richter) magnitude $\left(\mathrm{M}_{\mathrm{L}}\right)$, Nuttli magnitude $\left(\mathrm{Mb}_{\mathrm{LG}}\right)$, surface wave magnitude $\left(\mathrm{M}_{\mathrm{s}}\right)$, and body wave magnitude $\left(\mathrm{M}_{\mathrm{b}}\right)$ scales, each estimated using specific features of ground motion data (see Bormann \& Dewey, 2012 and Stein \& Wysession, 2003). This has resulted in some magnitude-determination artifacts, such as differing results from different seismograms or scales, as well as large-event saturation effects (Kanamori, 1977). A numerical 
measure to represent earthquake size in terms of its seismic moment (energy) release was proposed by Hanks \& Kanamori (1979). Termed the moment magnitude ( $\mathbf{M}_{\mathrm{w}}, \mathbf{M}$, or simply $\left.\mathbf{M}\right)$, this measure was adapted to reflect values similar to the publicly familiar local (Richter) scale at small magnitudes and does not saturate at large magnitudes. Further, the seismic moment is theoretically independent of variables such as instrument type or source distance and is therefore better suited for comparison between regions and studies. For these reasons, moment magnitude has surpassed other scales to become the preferred measure of earthquake size in scientific studies. In the following chapters of this thesis, the moment magnitude will be referred to simply as the magnitude. Additionally, the bolded type $\mathbf{M}$ will be used to describe moment magnitudes recorded or converted by a catalogue, and the normal type $\mathrm{M}$ is used in equation form to describe moment magnitudes estimated from analyses (thresholds, boundaries etc.).

Moment magnitudes for sufficiently large events may be computed using established moment tensor solutions and catalogues available online (e.g. The Global Centroid-Moment-Tensor Project (CMT), www.globalcmt.org), while ground motion algorithms using low-frequency ground acceleration data or supplementary scales are more suitable for smaller events (Chen \& Atkinson, 2002; Chen, 2010; Atkinson et al., 2014). In some cases, smaller earthquakes are initially recorded using a convenient magnitude scale based on the waveform information available and then converted to moment magnitudes using empirical relations (e.g. Fereidoni et al., 2012). It is worth noting that most magnitude scales, including moment magnitude, are logarithmic. A unit increase in magnitude corresponds to nearly a 32-fold increase in total energy release.

\subsubsection{Earthquake Catalogues and the Gutenberg-Richter Relation}

The information logged by a network of seismic stations, specifically the date and time of event occurrences, geographic locations, magnitudes, magnitude scales, and estimated depths, is recorded within earthquake catalogues. The seismic history of a region documented by catalogues is critical for the probabilistic assessment of seismic hazard and other forms of scientific investigation, such as earthquake forecasting or mapping of fault-zones, and is therefore structured chronologically in table form to allow for easy data handling and manipulation (McGuire, 2004; Schorlemmer et al., 2004b). One of the most fundamental 
representations of earthquake catalogue data is the empirical frequency-magnitude distribution (FMD), which provides insight into the seismicity in terms of detectability, magnitude recurrence and scaling. The FMD plots the cumulative and non-cumulative frequencies of earthquake magnitude occurrence in log-linear space. In most cases around the world, where tectonic environments and seismic timescales vary widely, the complete portion of the cumulative FMD tends to follow the theoretical Gutenberg-Richter (GR) relation

$$
N(\geq M)=10^{a-b\left(M-M_{0}\right)} ; M \geq M_{0},
$$

where $N(\geq M)$ is the cumulative number of detected events greater than or equal to magnitude $M, a$ reflects the level of seismicity over the time period considered (i.e. $N\left(\geq M_{0}\right)=10^{a}$, where $M_{0}$ is a baseline value), and $b$ describes the exponential scaling of the distribution, represented as a linear slope in semi-logarithmic space (Gutenberg \& Richter, 1944; Richter \& Gutenberg, 1956). Most seismically active areas as well as global seismicity appear to obey the GR law with $b$-value near unity (Imoto, 1991; El-Isa \& Eaton, 2014), implying that earthquake occurrence-frequency generally decreases by about a factor of 10 for each unit increase in magnitude. While the GR relation is perhaps the most widely-used method to describe frequency-magnitude statistics, alternate approaches and modifications have been suggested such as the cumulative normal (error) function (Woessner \& Wiemer, 2005; Iwata, 2008) or Bayesian generalizations of the GR (e.g. Sánchez \& Vega-Jorquera, 2018).

The broad applicability of the GR model allows for its parameters and their spatiotemporal variation to help characterize seismicity recorded within a catalogue, assess potential future occurrence, and be utilized in statistical models of greater complexity. Frequency-magnitude statistics are not only essential in the recurrence estimations of seismic hazard, but also in studies of earthquake rate analysis, cluster analysis, earthquake mechanics and seismic forecasting (Cornell, 1968; Baiesi \& Paczuski, 2004; Schorlemmer et al., 2004b; Wiemer \& Schorlemmer, 2007; Zaliapin et al., 2008; Mignan, 2011, 2012; Ordaz \& Faccioli, 2018). A pressing question in the ongoing investigation of induced seismicity is to what degree inducedearthquake characteristics and scaling relationships differ from those of natural events. Analysis of FMDs using the GR model may provide some insight into this problem. High $b$-values have been linked to seismic swarms and certain cases of induced seismicity (Rutledge et al., 2004; 
Vermylen \& Zoback, 2011; Guest et al., 2014). On the other hand, low-to-moderate $b$-values have also been observed, particularly in studies analyzing earthquakes related to wastewater injection (Holland, 2013; Huang \& Beroza, 2015; Skoumal et al., 2015; Ogwari \& Horton, 2016). Further, the scaling parameter $b$ has been shown to relate to other properties of the seismicity and surrounding environment, such as the differential and effective stress of the local crust (Schorlemmer et al., 2004a; Wyss et al., 2005; Goertz-Allmann \& Wiemer, 2013; El-Isa \& Eaton, 2014) and pore pressure conditions (Bridges \& Gao, 2006; Farrell et al., 2009; van Stiphout et al., 2009; Bachmann et al., 2012). Fluctuations in $b$ have been observed before large earthquakes (Imoto, 1991; Zuniga \& Wyss, 2001; Chan et al., 2012; Nuannin et al., 2012; Prasad \& Singh, 2015) and regional variations have been used to identify higher-risk zones more likely to result in fault rupture (Wiemer \& Wyss, 1997; Montuori et al., 2010). The comparison of GR parameters between studies should be done with caution, however, as values can range substantially based on the methods chosen for the determination of catalogue completeness (discussed below), the techniques used to fit $b$-values (such as a least-squares fitting of the FMD and the Aki-Utsu maximum likelihood estimation, discussed in Chapter 2.2), and the magnitude scales reported in the catalogue. Significant parts of this crucial information are often not considered or conveyed in the literature (Sandri \& Marzocchi, 2003; Nava et al., 2017a, 2017b, 2018).

\subsubsection{Magnitude of Completeness}

An important aspect of earthquake catalogues to consider is the magnitude of completeness $\left(M_{c}\right)$, which is, for a given space-time volume, the threshold magnitude above which all events are detected and logged by the seismic network (Rydelek \& Sacks, 1989). Network coverage is rarely uniform over a given region, as stations tend to be concentrated around critical infrastructure or in areas more prone to earthquakes, and may be added to or removed from the network based on necessity and changes in available resources. In addition, earthquake detection is dependent on site conditions (the geologic settings where the stations are located), ambient noise (auxiliary ground motion due to external factors like automobile traffic, industrial activities, subsurface fluid migration etc.) and the signal processing methods used by each station. For these reasons, $M_{c}$ itself tends to vary across space and time. The majority of earthquake statistics, including the parameters of the GR relation and subsequent risk analysis 
(McGuire, 2004; Ordaz \& Faccioli, 2018), but also aftershock statistics (Ogata \& Shimazaki, 1984; Ogata, 1988) and measurement of seismic transients, quiescence, triggering phenomena (Stein, 1999; Kilb et al., 2000; Wiemer \& Wyss, 2000) are directly impacted by the determination of this quantity, as they must be estimated from the complete portion of catalogue data. Overestimating $M_{c}$ would result in a loss of usable data and shrinkage of sample size, thereby increasing error, while underestimating it could bias the data and lead to erroneous results.

Many methods exist to estimate completeness, and may be broadly separated into two categories: network-based and catalogue-based methods. Network techniques include analysis of seismic waveforms (Schultz et al., 2015b), amplitude thresholds (Gomberg, 1991), day/night time network sensitivity (Rydelek \& Sacks, 1989), signal-to-noise ratios (Sereno \& Bratt, 1989; Harvey \& Hansen, 1994) and event-to-station distance (Wiemer \& Wyss, 2000; Mignan, 2012; Cui \& Atkinson, 2016). Some of these techniques can be advantageous for regional studies in areas characterized by infrequent coverage or low seismic activity, such as the WCSB, because they do not depend on bulk seismic data. For example, Schultz et al. (2015b) analysed noise characteristics at individual stations and performed synthetic tests on simulated earthquake data to measure the performance of both the Canadian National Seismic Network (CNSN) and the composite catalogue compiled by the Alberta Geological Survey (AGS) in 2010. Minimum magnitude thresholds, in the form of spatial $M_{c}$ contours, were determined across Alberta by the successful picking of $P$-phases from at least four stations (typically, an event is recorded by the network if it is detected by at least four stations). Another study by Cui \& Atkinson (2016) used a derived linear equation for $M_{c}$ based on event magnitudes and their maximum detectable distance in order to conduct a spatiotemporal evaluation of the detection capability across Alberta. Contour maps of $M_{c}$ were produced, similar to Schultz et al. (2015b), for a series of time periods as the collective networks went through substantial changes and steady improvements over time. Both studies mapped completeness after 2010 at higher values towards the northern extremities of the region $\left(M_{c} \geq 3\right.$ above $58^{\circ}$ latitude), whereas in central and southern Alberta, particularly near the previous earthquake clusters close to Rocky Mountain House and Cardston, completeness estimates were reduced substantially $\left(M_{c} \leq 2\right)$. 
Catalogue-based techniques to estimate $M_{c}$ include those that directly analyze the cumulative and non-cumulative FMDs of the seismicity, such as the method of maximum curvature, goodness-of-fit test (Wiemer \& Wyss, 2000), and the GR $b$-value stability approach (Cao \& Gao, 2002), as well as techniques evaluating other statistical parameters of the catalogue, such as Bayesian statistics (Mignan et al., 2011; Mignan \& Chouliaras, 2014) and the harmonic mean of magnitudes (Godano, 2017). Many of these methods rely on the grounded assumption that the GR relation holds for the entire magnitude range of interest; specifically that the occurrence of earthquakes continues to scale according to Equation [1.1] for both larger events that may be under sampled over the timescale considered as well as for smaller magnitudes below the level of completeness. There is evidence that suggests the GR law is maintained down to pico-seismicity (Abercrombie \& Brune, 1994; Kwiatek et al., 2011). $M_{c}$ is taken as the magnitude beneath which the empirical cumulative FMD departs from the theoretical scaling. An adequate dataset is necessary to apply these techniques due to their direct dependence on the sampling statistics of the catalogue. Studies agree that some minimum number of events should be required when computing the $b$-value, and consequently $M_{c}$, from the GR distribution; estimates using fewer than 50 events are unfortunately not uncommon among seismicity studies, and have been shown to be practically meaningless given the size of their standard errors (Nava et al., 2017a, 2017b, 2018). For these reasons, catalogue techniques are generally less reliable in regional mapping studies of $M_{c}$, where earthquake density varies and FMDs must be constructed with some population bias, but on the other hand can be advantageous in analyses of specific subsets of a catalogue, such as seismic clusters or pockets of significant activity, where the FMD is plotted with only the relevant data and $M_{c}$ may be estimated efficiently.

\subsubsection{Earthquake Clustering}

Due to the lack of precise knowledge of the innumerable factors involved in the tectonic earthquake process, the occurrence of natural "background" earthquakes may be approximated as random and modeled as a time-stationary, space-inhomogeneous marked Poisson process (SIP). Within this framework, rates of seismicity are assumed to vary in space but not in time and data points (seismic events) are marked by their magnitudes. In reality, however, the occurrence of earthquakes is not a stationary or independent process and therefore cannot be 
fully represented by a Poisson model. In other words, the rate of all earthquakes in a given region is not constant and depends in part on earthquakes that have occurred beforehand (Mogi, 1963; Ogata, 1988; Kilb et al., 2000; Lombardi et al., 2010; Boyd, 2012; Mignan et al., 2018). This may be observed most clearly in the form of clustering, where seismic events are concentrated close together spatiotemporally. Earthquakes deviating from Poisson behavior are observed in most seismically active regions, and occur naturally around plate boundaries, within fault structures and near fluid-rich settings, such as volcanic or geothermal sites (Ogata et al., 1996; Hainzl, 2004; Vidale \& Shearer, 2006; Zaliapin \& Ben-Zion, 2013a). Perhaps the most common and well-understood type of cluster is the aftershock sequence, where the local seismicity rate temporarily increases after a large event (a mainshock) due to the transfer of stress and brittle failure of the crust (Hill et al., 1990; Stein, 1999). The rates of many large aftershock sequences appear to decay exponentially, and have been observed to obey the Omori-Utsu law (discussed in Chapter 2.4) (Omori, 1894; Utsu, 1961). Clustering can also occur in the more ambiguous form of a seismic swarm, where a sequence of events is not attributable to any dominant mainshock, and whose spatial and temporal extent instead seems to depend on the redistribution of stress caused by each subsequent event and/or external factors, such as fluid intrusion (Hainzl, 2004; Zaliapin \& Ben-Zion, 2013b). Other forms of clustering include smaller bursts, often caused by local elastic fault failure, and foreshocks, which are seismic events that precede a mainshock and potentially travel along asperities within the main fault surface (Jordan et al., 2005). Cases of induced seismicity commonly occur as clusters and appear to manifest as both foreshock-mainshock-aftershock burst sequences and as seismic swarms. For example, the wastewater disposal-induced M5+ events in Fairview, Prague, and Pawnee, Oklahoma all triggered typical aftershock behavior (Keranen et al., 2014; McGarr \& Barbour, 2017), while the HF-induced clustering near Youngstown, Ohio and injection-related events in central Arkansas near Guy and Greenbrier displayed swarm-like characteristics (Horton, 2012; Llenos \& Michael, 2013; Skoumal et al., 2015).

The intensity and nature of an earthquake cluster is typically related to a combination of factors including the local geologic setting, stress field, fault orientation, and previous earthquakes (Scholz, 2002). In addition, many studies have shown that both natural and anthropogenic changes to the subsurface fluid content can enhance or induce earthquake sequences via 
subsidence and/or changes in Coulomb fault stress and pore pressure conditions, especially near critically oriented structures (Segall, 1985; Langenbruch \& Shapiro, 2010; Brodsky \& Lajoie, 2013; Kumazawa \& Ogata, 2013; Keranen et al., 2014; Goebel et al., 2015; Schoenball et al., 2015; Bao \& Eaton, 2016). The nature of clustering observed within a region may be attributed to its rheological structure, in the framework of viscoelastic deformation (Ben-Zion \& Lyakhovsky, 2006). In this context, a medium with low levels of heat flow and/or less fluid content correlate with higher viscosity and the conditions of brittle rheology, resulting in "burstlike" cracking of the crust and subsequent aftershock clustering. The converse is attributed to lower viscosity lithospheres of more brittle-ductile rheology (higher levels of heat and/or more fluid content), where failure is more likely to result in swarms of inter-linked events related to factors such as local fluid balance and destabilizing aseismic slip (Zaliapin \& Ben-Zion, 2016; Scuderi et al., 2017; Martínez-Garzón et al., 2018).

Earthquake cluster identification within a catalogue involves a separation of the independent background rate from dependent event sequences (Gardner \& Knopoff, 1974; Reasenberg, 1985; Baiesi \& Paczuski, 2004; Console et al., 2010; Ader \& Avouac, 2013; Zaliapin \& BenZion, 2013a; Schaefer et al., 2017). This is an important and non-trivial task required not only in cluster analysis but also in seismic hazard assessment, where catalogues must be de-clustered in order to delineate different source zones of the spatially inhomogeneous background and assess recurrence parameters. Recently, studies have suggested that the hazard of dependent seismicity in the form of foreshocks and aftershocks be included rather than removed, as spatiotemporally correlated events have been shown to increase hazard non-linearly (Boyd, 2012; Mignan et al., 2018). In any case, the practical separation of clustered and background seismicity should not restrict the consideration for potential interplay between them, particularly when external factors, such as anthropogenic activity, are involved. Induced seismicity has been observed to increase both the background rate and clustering productivity within affected regions (Lombardi et al., 2010; Llenos \& Michael, 2013; Schoenball et al., 2015; Maghsoudi et al., 2016, 2018; Zaliapin \& Ben-Zion, 2016; Vasylkivska \& Huerta, 2017; Martínez-Garzón et al., 2018); it is plausible that a rise in the former subsequently affects a rise in the latter. 
Zaliapin \& Ben-Zion (2013a, 2013b) analyzed multiple southern California earthquake catalogues, which contain a large amount of both natural (tectonic and magmatic) and manmade (geothermal energy production-related) seismicity, using the Nearest-Neighbour Distance (NND) method (discussed in detail in Chapter 2.5). Briefly, the NND approach links events to their closest ancestor, i.e. their "nearest-neighbour", based on a space, time, and magnitudedependent metric $\eta$, which is parameterized by the GR $b$-value and the dimension of eventepicenter distribution. Events are separated into clusters (those that are "strongly" linked to their nearest-neighbour) and background seismicity (those that are only "weakly" linked), whereby variation in relative mixing proportions between the two populations may be identified. Clusters may then be classified further into mainshock-aftershock "burst-like" sequences or inter-event triggered "swarm-like" sequences using their distinguishable structural characteristics. Zaliapin \& Ben-Zion (2013a, 2013b), along with Hicks (2011), found that a natural separation in the rescaled distance metric $\eta$ occurs between clustered and background events for many regional catalogues, as well as for worldwide seismicity. This separation cannot be explained by marginal spatial or temporal distributions, but instead appears to be a dependent feature of the unique spatiotemporal structuring of event clusters manifesting directly from the background and/or external triggers. Furthermore, the authors found that events within the clustered mode largely exhibit characteristics of either burst or swarm-like sequences. Their studies agreed well with the viscoelastic damage model, where a higher degree of inter-event triggering or swarm-like clustering was found within more ductile regions, such as geothermal settings or areas prone to magmatic or dike intrusion (Sagiya et al., 2002; Morita et al., 2006; Farrell et al., 2009), whereas more brittle rheology tended toward a higher proportion of individual events or rapidly decaying burst-like sequences (Zaliapin and BenZion 2013b).

Llenos \& Michael (2013) characterized both natural and fluid-injection induced swarms in Arkansas, namely the natural 1980s Enola sequence and the wastewater disposal-induced 20102011 Greenbrier sequences, by applying the Epidemic Type Aftershock Sequence (ETAS) model (Ogata, 1988, 1989; Zhuang et al., 2004) (discussed in detail in Chapter 2.4). The ETAS model estimates the time dependent seismic rate using the summation of a constant background term with a parameterized Omori-type aftershock kernel. This model is typically applied to 
catalogued seismicity and individual sequences to model event frequency across time, where both the quality of fit and the optimized parameters can be assessed and compared (Hainzl \& Ogata, 2005; Lei et al., 2008). Llenos \& Michael (2013) found that the Enola and Greenbrier swarms could not be modeled using the same set of parameter values, with the induced cluster resulting in both a higher background rate and elevated aftershock productivity relative to the natural swarm. Significantly smaller spatial distances between subsequent events during the induced swarm were also observed, suggesting something fundamentally different occurring in the subsurface triggering mechanics between the two sequences. The authors proposed that variation in the absolute values of ETAS parameters may be a way to distinguish between natural and human-induced seismicity within the same region, particularly changes in the background rate parameter $\mu$ and magnitude-weighting factor $\alpha$ (see Chapter 2.4).

Maghsoudi et al. (2018) performed statistical tests on micro-seismic catalogues to differentiate between induced seismic clustering driven directly by hydraulic fracturing and that which has been triggered by other earthquakes. HF operations were found to elevate background seismicity directly, which could stimulate inter-event triggered clustering deviating from the Poisson process. This implies that there were different mechanisms driving the microearthquakes initiated by the rock-fracturing operation and associated fault structure changes versus those caused by previous events. The micro-seismic event triggering essentially followed the Omori-Utsu aftershock relation (see Chapter 2.4) and could also lead to spatiotemporal clustering independent of the fluid injection rate. The authors emphasized that seismic activity induced by HF operations appears to scale not only with injection volume and pressure, but may also be dependent on subsequent inter-event triggering following a rise in background event occurrence.

\subsection{Thesis Structure}

The global rise in induced seismicity together with advancement in detection capability over the past decade has resulted in an abundance of earthquake data from which much new information is being gathered (Doglioni, 2018). Better understanding of tectonic strain rates, triggering mechanisms and geologic controls, updated hydrogeological models, and analyses of spatiotemporal clustering patterns and statistics have allowed for some improvements in the identification of induced activity and several adjustments to seismic hazard analysis (Llenos \& 
Michael, 2013; Atkinson et al., 2016; Petersen et al., 2016; Zaliapin \& Ben-Zion, 2016; Atkinson, 2017; Langevin et al., 2017; Schoenball \& Ellsworth, 2017; Kao et al., 2018; Zhang et al., 2019). However, the wide-ranging and sometimes conflicting results attained across individual case studies (see Keranen \& Weingarten, 2018) highlight the complex nature of induced seismic processes and illustrate the need for further investigation.

The absence of some fundamental and reputable discriminatory characterization between natural and induced activity, and between earthquakes triggered by different types of inducing mechanisms offers significant motivation for this study. In western Canada, the concerning profusion of isolated earthquake clusters has nonetheless provided an opportunity for detailed cluster analyses and possible statistical comparison. This work aimed to analyze the regional and clustered seismicity statistically and identify distinguishing characteristics arising from the application of three models, namely the Gutenberg-Richter (GR), Nearest-Neighbour Distance (NND) and Epidemic Type Aftershock Sequence (ETAS) models.

In Chapter 2, the seismic dataset and statistical methods utilized in this study are described in detail. The techniques used to apply the GR model to frequency-magnitude distributions and estimate the productivity ( $a$-value) and scaling ( $b$-value) parameters are reviewed. Three catalogue methods for the estimation of local completeness magnitudes are discussed, which were used to accurately compute the GR and ETAS parameters. Finally, the stochastic point process ETAS model and the correlated-event linking NND method are described, as is the methodology behind interpreting and comparing the results.

The statistical analysis portion of the thesis is split into two parts: a regional study and an individual cluster study. Chapter 3 provides the results of the regional study, where the NND model is applied to all seismicity recorded within the Composite Alberta Seismicity Catalogue (CASC, discussed in Chapter 2) over specified time intervals both predating and during the widespread implementation of hydraulic fracturing (HF). The main objectives of this chapter are to highlight observed statistical differences between natural and induced activity as well as identify changes in earthquake distributions across time. Chapter 4 provides the results of the individual cluster study where analysis is performed on prominent earthquake clusters within the WCSB, namely the production-related clustering near Rocky Mountain House, the 
wastewater disposal and HF-related earthquakes along the Montney trend and the recent HFstimulated clustering near Fox Creek. The objectives in Chapter 4 are twofold; the first is to observe the frequency-magnitude scaling within each isolated cluster and compute estimates for completeness magnitudes $\left(M_{c}\right)$ and the GR parameters. Many other studies have typically relied upon a single catalogue method to estimate $M_{C}$ when conducting GR analyses and hence may have been prone to the biases demonstrated by Huang et al. (2016) (detailed in Chapter 2.3); by using a suite of tests I aim to achieve more robust estimations. The second objective is to observe the clustering behavior statistically and categorize the sequencing through the application of the NND and ETAS models.

In summary, background information is provided on the event catalogue and statistical methods and models used (Chapter 2), results of analysis and discussion for nearest-neighbour distance distributions of the regional catalogue over time are given (Chapter 3), results and discussion of the evaluation of local $M_{c}$, GR parameters and subsequent application of the NND and ETAS models to individual clusters are presented (Chapter 4). Finally, conclusions and potential future directions are summarized (Chapter 5). 


\section{Chapter 2}

\section{Data and Methods}

\subsection{Earthquake Catalogue}

The Composite Alberta Seismicity Catalogue (CASC), available online at www.inducedseismicity.ca, contains seismic event records from the early 1900s through to the present (Figure 2-1). The CASC is compiled from several contributing agencies operating across Alberta and eastern British Columbia, including the Geological Survey of Canada and Earthquakes Canada (www.seismo.nrcan.gc.ca), the Alberta Geological Survey and the Regional Alberta Observatory for Earthquake Studies (ags.aer.ca), the Canadian Rockies and Alberta Network (‥iris.edu), and the TransAlta/Nanometrics Network (www.nanometrics.ca). The catalogue is constructed as a data table, with entries containing the date and time, estimated geographic location, magnitude, magnitude scale, and moment magnitude conversion for each detected event occurrence (for a detailed discussion on the compilation of the CASC, see Cui et al., 2015). The database is estimated to be complete to the $M_{c}=3$ level from 1985-2018 (Adams \& Halchuk, 2003; Cui \& Atkinson, 2016). As mentioned in Chapter 1.3.3, seismic network coverage is generally spatiotemporally inhomogeneous and so local completeness levels over a given time period may be substantially lower than the regional completeness; this matter is explored in the cluster analyses in Chapter 4. Accurate depth estimations remain a difficult task for most networks and, as a consequence, depths listed in this catalogue have large errors or are only specified as default values. While hypocentral locations would be beneficial for statistical analyses in three dimensions, they are not critical. The methods used in this thesis require relatively few input requirements; only the magnitudes, epicentral locations and times of occurrence were used from the database.

Some potential artifacts related to catalogue inconsistencies are worthwhile to note. First, many of the seismic recordings within the CASC are nontectonic and instead a product of quarry and mining blasts. These events are generally flagged by network personnel based on several criteria, including event time (blasts occur during daylight hours), proximity to active mines and quarries, and specific waveform characteristics (typically compressional first motions and high frequency spectra) (Cui et al., 2015; Schultz et al., 2015b; Cui \& Atkinson, 2016). In this 
thesis, all flagged events were removed from the catalogue prior to the statistical analyses detailed in the following chapters. However, recent blast events (after 2014), southwest of Calgary, had not yet been flagged by the network at the time of access (last accessed May 2019, $\underline{\text { www.inducedseismicity.ca) }}$ and were hence included in the analyses. A second potential artifact is that the CASC is spatially limited to the Alberta region, particularly south of $53^{\circ} \mathrm{N}$. This explains the lack of recorded events surrounding the Vancouver area (Figure 2-1). Readers interested in documented seismicity west of Alberta are referred to the National Earthquakes Database (NEDB; database link).

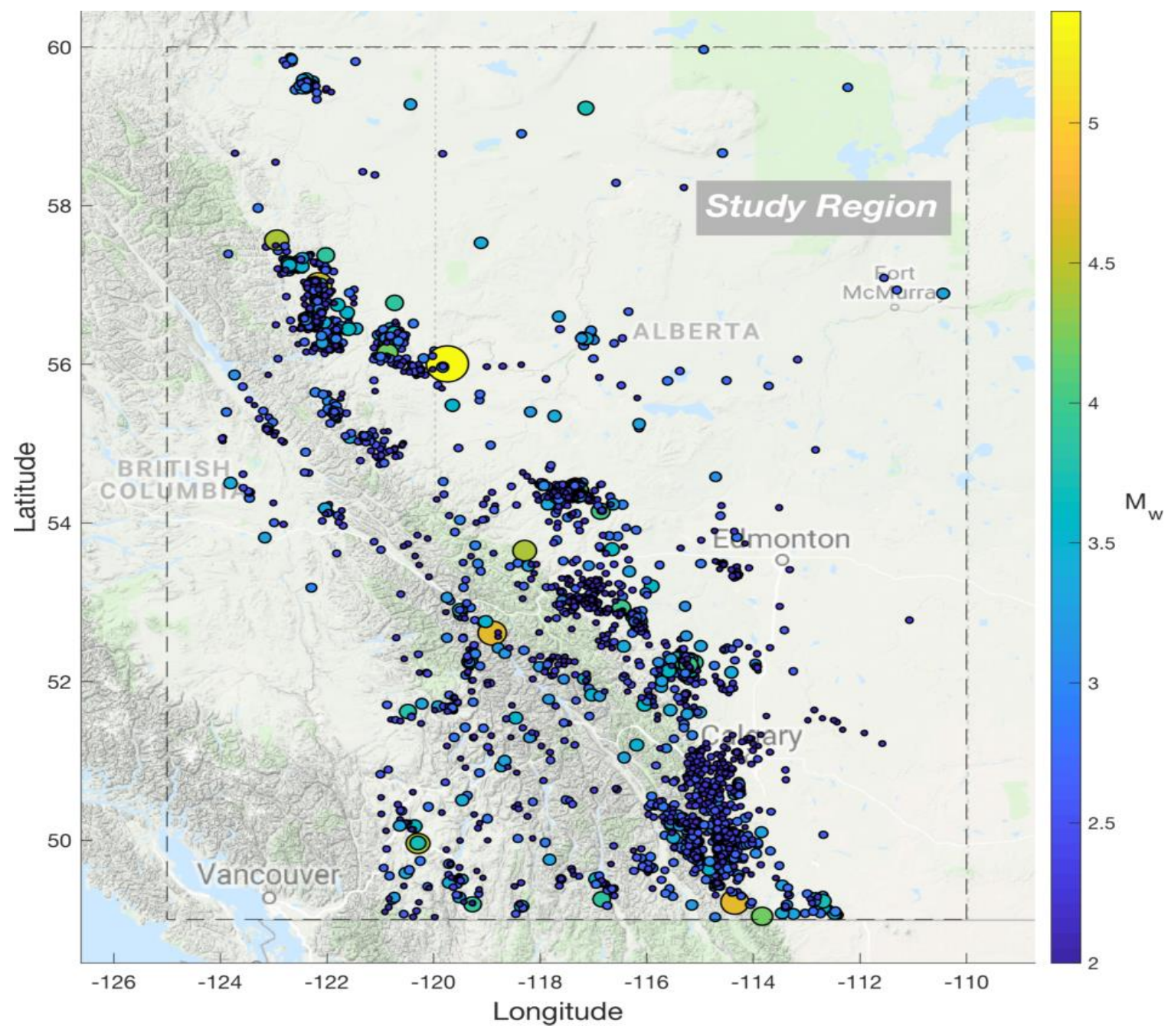

Figure 2-1: Map of $M \geq 2$ seismic events documented by the Composite Alberta Seismicity Catalogue (CASC) from 1975-2018. Marker size and colour indicate earthquake magnitude. 


\subsection{Estimation of the GR Parameters}

The Gutenberg-Richter (GR) relation models the frequency-magnitude statistics of an earthquake dataset as an exponential distribution. Its applicability to both natural and induced earthquakes across a broad range of tectonic settings makes it an effective tool for characterizing seismicity (Sandri \& Marzocchi, 2003; El-Isa \& Eaton, 2014). Further, the validated assumption of GR scaling for magnitudes greater than or equal to a cut-off value $M_{c}$ provides an opportunity to estimate completeness. The GR model was applied as a part of the cluster analyses in Chapter 4, first to estimate the local completeness magnitude using three catalogue-based techniques (detailed in the following subsection) and second to estimate the productivity and recurrence-scaling parameters $a \& b$. The $b$-values were then used to parameterize and apply the NND model (see Chapter 2.5).

The maximum likelihood estimation (MLE), known as the Aki-Utsu method, was utilized to compute the GR parameters in this study. MLE techniques make use of the likelihood function to determine parameter values that maximize the probability of drawing the given data sample. The combination of this set of parameters exists at the peak of the likelihood function surface, which is defined over the model parameter space. The Aki-Utsu method utilizes the magnitude sampling average $\mu$ of the dataset in order to estimate the $b$-value for both continuous and grouped data (Aki, 1965). Fitting the GR model via a linear (least squares) fitting of the semilogarithmic FMD was also possible, however it has been shown that this method disproportionately weights the largest magnitude events in the distribution (Tinti \& Mulargia, 1987; Felzer, 2006). Equation [1.1] implies that, according to the GR relation, event magnitudes are distributed exponentially with the probability density function

$$
f(M)=\beta e^{-\beta\left(M-M_{0}\right)} ; M \geq M_{0}, \beta>0 .
$$

$M$ is the earthquake magnitude, $M_{0}$ is the minimum/cut-off magnitude considered (equivalent to the level of completeness $M_{c}$ ) and $\beta=b \ln 10$. Aki (1965) showed that the MLE for $\beta$ based on a continuous magnitude distribution is

$$
\beta=\frac{1}{\mu-M_{0}}
$$


where $\mu$ is the mean magnitude (sampling average for all magnitudes above or equal to $M_{0}$ ) of the dataset. In reality, however, catalogued magnitudes are not sampled from a continuous distribution and instead are estimated by a network. Events are generally rounded to the 0.1 magnitude unit, though historical earthquake datasets binned into 0.5-0.6 unit intervals are not uncommon. This rounding process indicates that the sampling average $\mu$ will be biased, as the actual minimum magnitude threshold is equivalent to $M_{0}$ less half the binning interval. Utsu (1966) introduced a correction for the MLE of $\beta$ based on a discrete magnitude distribution, which accounts for this uncertainty in instrumental magnitude measurement as

$$
\beta=\frac{1}{\mu-\left(M_{0}-\frac{\Delta M}{2}\right)},
$$

where $\Delta M$ is the width of the magnitude bin. Thus,

$$
b=\frac{1}{\ln 10\left[\mu-\left(M_{0}-\frac{\Delta M}{2}\right)\right]} .
$$

Bender (1983) showed that when $\Delta M$ is small, the maximum likelihood formulas for continuous and grouped data are indistinguishable.

By taking the logarithm of Equation [1.1], the GR relation may be plotted in log-linear space as

$$
\log _{10} N(\geq M)=a-b\left(M-M_{0}\right) ; M \geq M_{0} .
$$

Since $M_{0}$ is established beforehand and $b$ is determined by Equation [2.4], $a$ may be solved for directly.

It is clear that the estimates attained by Equation [2.4] and Equation [2.5] will approach their true values only if $\mu$ is representative of the distribution. In other words, the sampling average must represent the sample within some acceptable standard error, which is dependent upon the appropriate choice of $M_{0}$ (discussed in the next subsection) and the size of the dataset. Shi \& Bolt (1982) and Tinti \& Mulargia (1987) derived these confidence limits for grouped magnitude data (Equation [2.7]), which are contextually important when estimating and 
comparing GR parameters (e.g. Nava et al., 2018). The $95 \%$ confidence limits for all parameter estimates are provided in the cluster analyses in Chapter 4.

\subsection{Catalogue-based Methods for the Estimation of $M_{c}$}

The magnitude of completeness $\left(M_{c}\right)$ is defined as the minimum magnitude above which all events are detected by a seismic network (Wiemer \& Wyss, 2000). Accurate calculation of seismic statistics, including the GR parameters (discussed above) and the ETAS parameters (discussed in Chapter 2.4), is directly dependent upon the correct choice of $M_{c}$. Overestimating it would result in a loss of usable data and shrinkage of sample size (though deliberately varying the cut-off above $M_{c}$ can be a useful exercise during seismic analysis e.g. Shcherbakov et al., 2004, 2005b, 2005a). Underestimating $M_{c}$ could bias results, for example by improperly weighting the magnitude sampling average $\mu$ in Equation [2.4]. Several catalogue-based methods have been introduced in the literature to identify $M_{c}$ as the point of departure of the cumulative FMD from the theoretical GR distribution (the straight line in semi-logarithmic space given by Equation [2.5]). Below this cut-off point, the cumulative count tails off where smaller magnitude events are underreported. Three catalogue-based methods to estimate local $M_{C}$ values were applied in the cluster analyses in Chapter 4 and are described below.

\subsubsection{Method of Maximum Curvature (MAXC)}

The MAXC method was proposed by Wiemer \& Wyss (2000) as a rapid and stable method of estimating completeness. They defined $M_{c}$ as the point of maximum curvature of the cumulative FMD, i.e. where its first derivative is maximized. For a given dataset, this value corresponds to the magnitude bin containing the largest portion of events in the non-cumulative FMD (Figure 2-2). Despite this method's attractiveness from an efficiency standpoint it has been found to underestimate the completeness level, particularly in areas characterized by erratic network coverage resulting in curved or stepped FMDs (Wiemer \& Wyss, 2000). On the other hand, for smaller datasets it appears to be more stable than some other methods (Huang et al., 2016). 


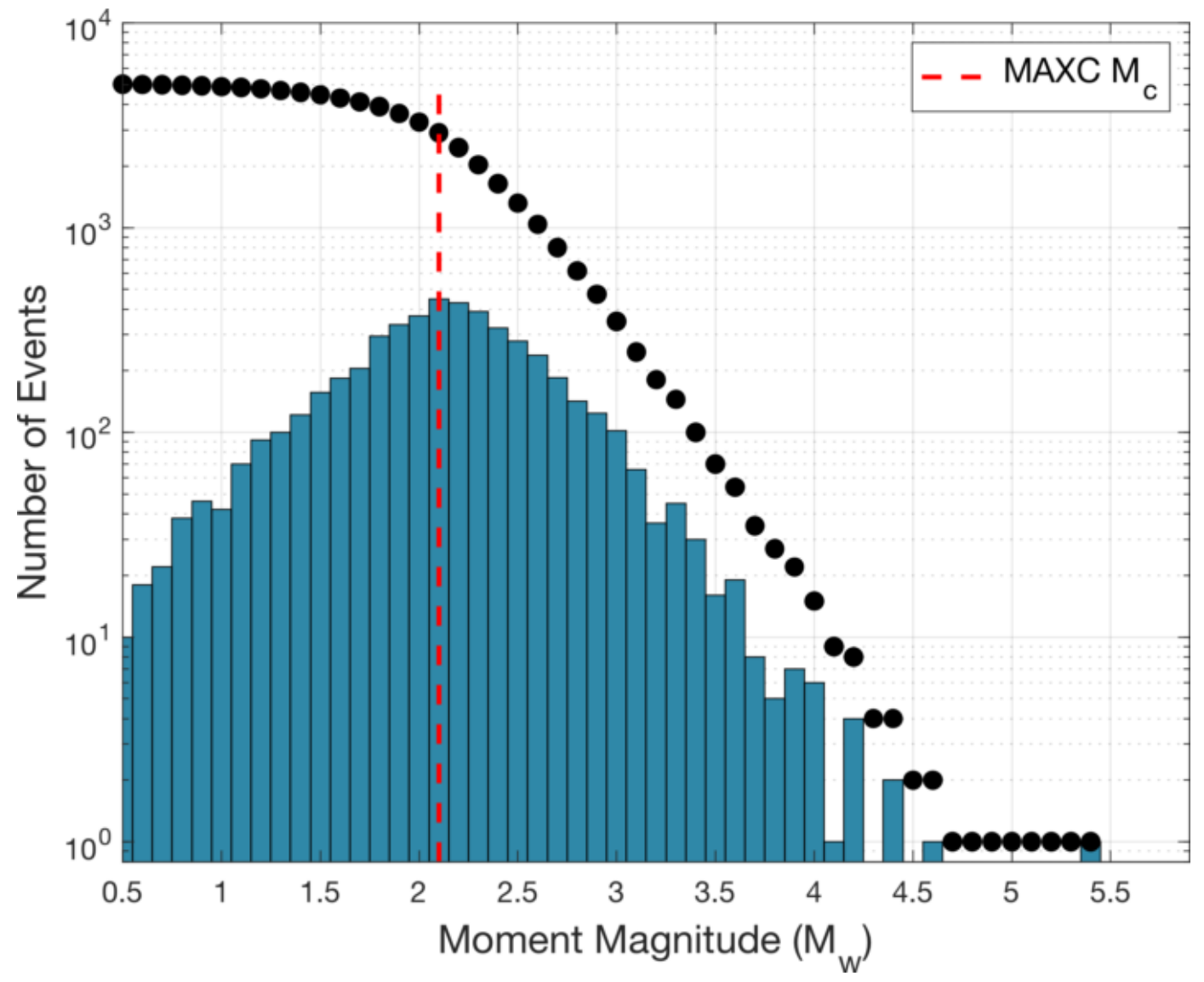

Figure 2-2: An example of the MAXC method applied to a sample frequency-magnitude distribution (FMD). Blue histogram represents the individual earthquake count per magnitude bin (non-cumulative FMD) and black dots represent the cumulative earthquake count per magnitude bin (cumulative FMD). Vertical dashed red line indicates the point of maximum curvature, which corresponds to the largest magnitude bin.

\subsubsection{Goodness-of-Fit Test (GFT)}

The GFT method was the second technique proposed by Wiemer \& Wyss (2000), based on the minimization of the residual between the observed cumulative FMD and synthetically generated distributions. The incomplete portion of a catalogue $\left(M_{i}<M_{c}\right)$ cannot be modeled by an exponential distribution, and hence the GFT residual value will be high for cut-off magnitudes below completeness. The normalized quantity $R$ represents the goodness-of-fit of a synthetic distribution to the empirical FMD as a confidence level, computed as 


$$
R\left(a, b, M_{0}\right)=100-\left(\frac{\sum_{i=M_{0}}^{M_{\max }}\left|B_{i}-S_{i}\right|}{\sum_{i} B_{i}} \cdot 100\right)
$$

Here $a$ and $b$ are the parameters of the GR distribution (calculated using the method detailed in Chapter 2.2 at the cut-off magnitude $M_{0}$ ). $B_{i}$ represents the number of events empirically observed within the magnitude bin $M_{i}$, while $S_{i}$ is the number of events expected to have occurred given a perfect power-law FMD governed by parameters $a$ and $b$. The second term in Equation [2.6] will be large if the differences between $B_{i}$ and $S_{i}$ are significant over the summation. Synthetic distributions and corresponding $R$-values are computed for a range of magnitude cut-offs; $M_{c}$ is taken as the magnitude at which $R$ exceeds some threshold value, typically $90-95 \%$ for a sufficient dataset.

It is possible that the choice of a threshold value as a cut-off point causes the GFT method to underestimate $M_{c}$, as cut-offs resulting in greater confidence above the threshold value are omitted (Huang et al., 2016). Furthermore, in this study, synthetic fits above $85 \%$ were rarely achieved due to the paucity of data and so establishing a fixed confidence value as a threshold was not possible. Instead, the procedure was altered slightly where the GFT $M_{c}$ was chosen as the magnitude for which the synthetic distribution best describes the empirical data i.e. when $R$ is maximized (Figure 2-3). 


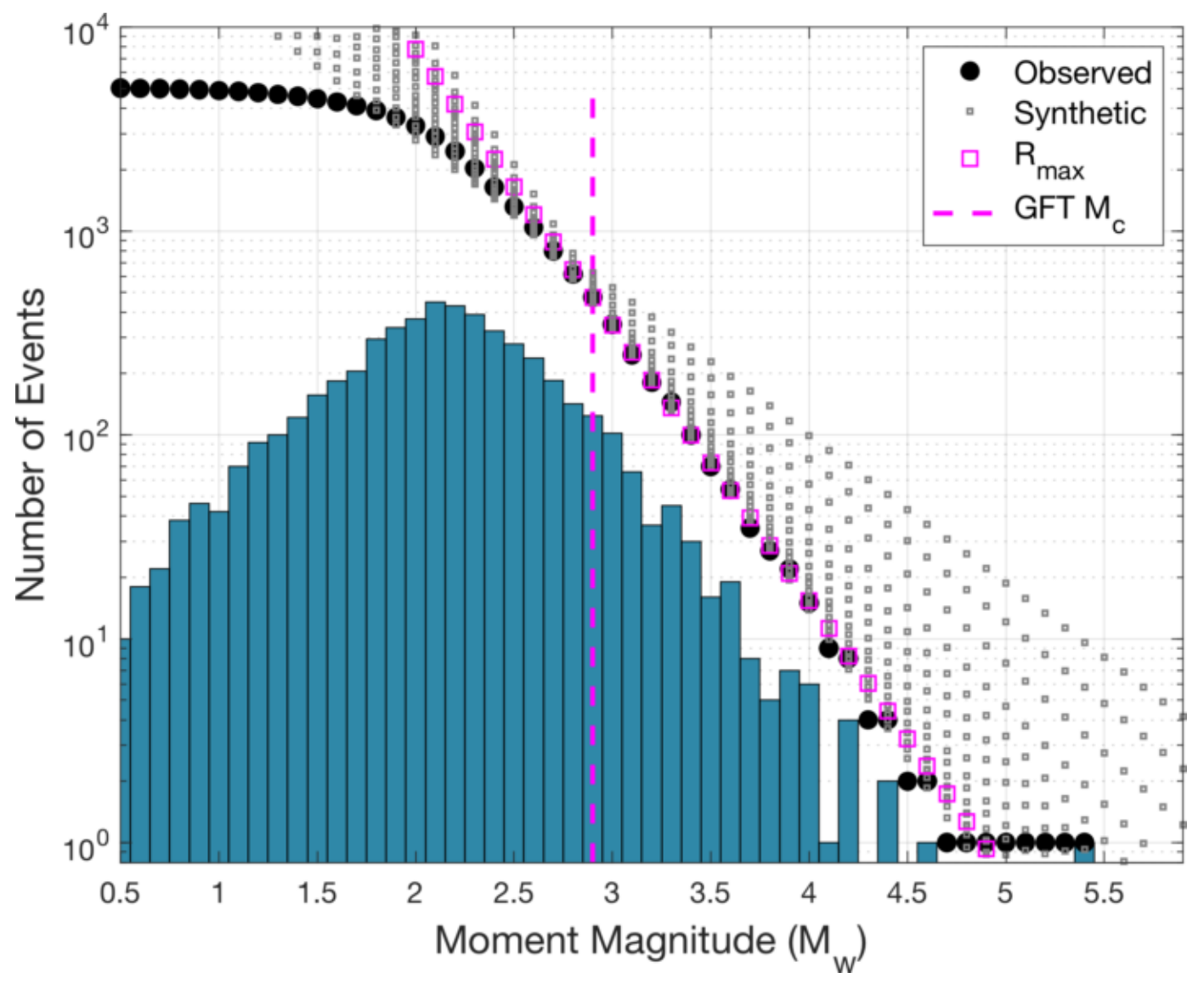

Figure 2-3: An example of the GFT method applied to a sample FMD. Pink squares represent the synthetic distribution that best describes the data. Grey squares represent the rejected synthetic distributions. Vertical dashed pink line indicates the corresponding GFT $M_{c}$.

\subsubsection{Method of $b$-value Stability (MBS)}

The MBS method is the third catalogue method used in this study for estimating completeness. It was first proposed by Cao \& Gao (2002), who estimated the varying completeness of the Tohoku University seismic network in north-eastern Japan. This technique is once again based on the observed self-similarity of the earthquake process illustrated by the GR relation. For cutoff magnitudes below completeness $\left(M_{i}<M_{c}\right)$, where the FMD tails off, the associated $b$ values will be low and unstable. As $M_{i}$ increases, the $b$-values grow and eventually stabilize once $M_{i} \geq M_{c}$. Further, once $M_{i}$ exceeds the magnitudes of adequate sampling, where $M_{i} \gg$ $M_{c}$, the corresponding $b$-values will theoretically increase again as the FMD drops off. 
Cao \& Gao (2002) computed $M_{C}$ as the first magnitude above a certain threshold for which the $b$-value changed by less than 0.03 units. However, analysis of only two subsequent magnitude bins can be unstable, particularly with sparser datasets. Woessner \& Wiemer (2005) introduced an alternative threshold, utilizing the average $b$-value across five successive magnitude bins $\left(b_{a v e}\right)$ and the uncertainty in $b$ of the first bin $(\delta b)$, calculated according to Shi \& Bolt (1982) as

$$
\delta b=2.3 b^{2} \sqrt{\frac{\sum_{i=1}^{N}\left(M_{i}-\mu\right)}{N(N-1)}} .
$$

In Equation [2.7], $N$ is the number of events greater than or equal to the chosen cut-off and $\mu$ is the magnitude-sampling average. $M_{c}$ is defined as the magnitude at which the absolute difference $(\Delta b)$ between the $b$-value of the first bin and $b_{a v e}$ is less than or equal to the uncertainty $\delta b$. This comparison with the rolling average over five consecutive values ensures that any successive stability is not coincidental. Huang et al. (2016) demonstrated that the MBS method is among the most conservative of catalogue-based techniques, however it requires a large enough dataset from which to compute a range of $b$-values with reasonable uncertainties. In this study, it was often the case that the rolling average $b_{\text {ave }}$ approached the $b$-value even when uncertainty was fairly large, and so where $\Delta b$ was minimized provided a suitable upper bound to $M_{c}$. Visual inspection of $b_{a v e}, \delta b$, and $\Delta b$ plotted alongside $b$ was used and $M_{c}$ was considered as bounded between where $\Delta b$ first dipped below $\delta b$ and where $\Delta b$ was minimized (Figure 2-4). 


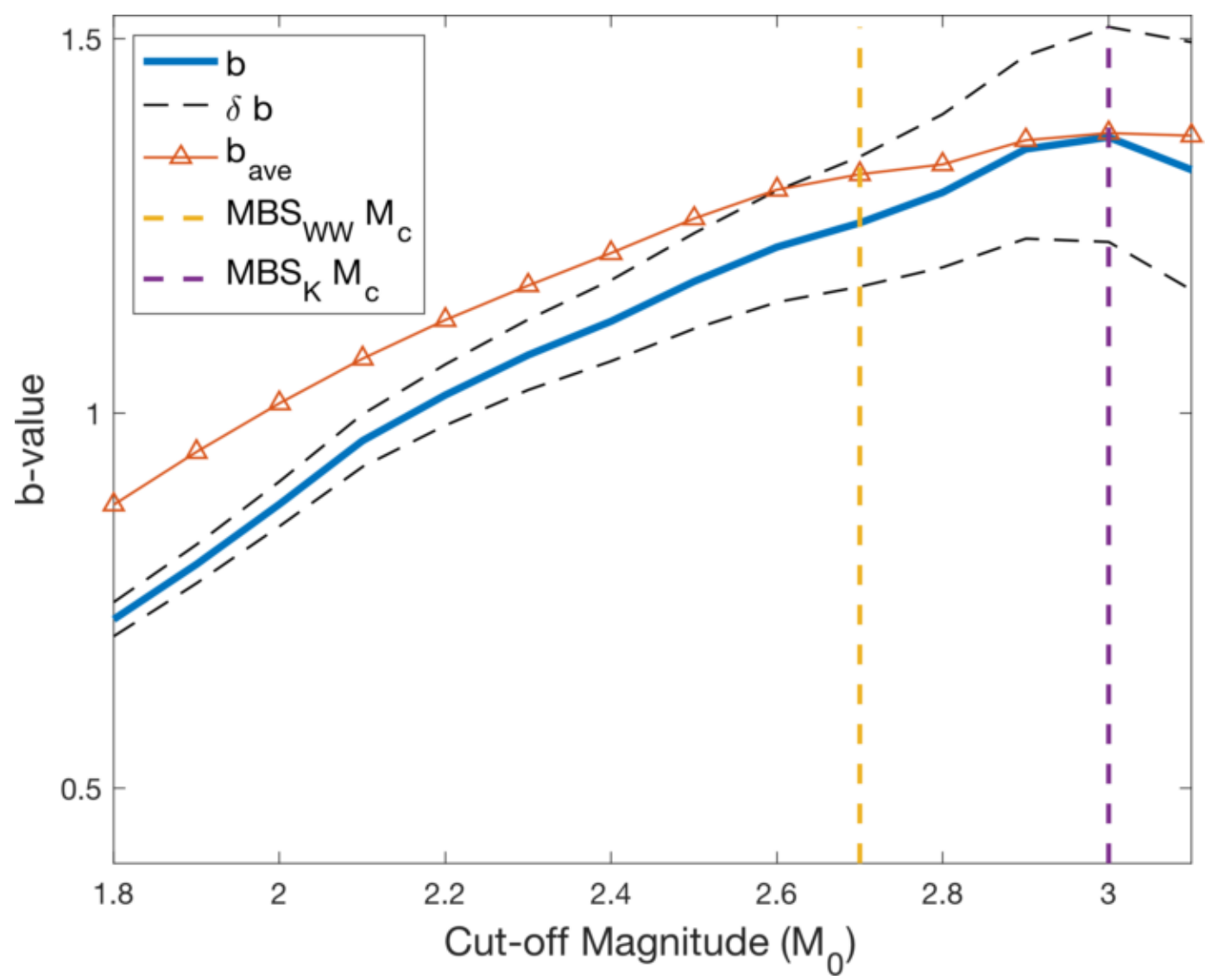

Figure 2-4: An example of the MBS method applied to a sample catalogue. Blue solid line represents the MLE $b$-values for a range of cut-off magnitudes and dashed black lines are the uncertainty. Orange line with triangle markers represents the rolling average $\left(b_{\text {ave }}\right)$. Vertical dashed yellow line indicates completeness as determined by Woessner \& Wiemer (2005) (where $b_{\text {ave }}$ first dips below uncertainty), which is considered as the lower bound for $M_{c}$ in Chapter 4. Vertical dashed purple line indicates where $\Delta b$ is minimized, which is considered as the upper bound.

\subsection{The Epidemic Type Aftershock Sequence (ETAS) Model}

The ETAS model, developed by Ogata $(1988,1989)$, is a branching-process model based on an expansion of the Omori-Utsu law of aftershocks (Omori, 1894). This model was utilized in Chapter 4 in order to assess and compare the time-varying rates of seismicity, aftershock parameters and model-fitting quality between individual cases of induced clustering. 
The original Omori law states that, after a mainshock at time $t_{0}$, the rate of aftershocks decays as

$$
\lambda(t) \approx \frac{1}{t}
$$

Here $t$ is the time elapsed since $t_{0}$. Utsu (1961) introduced an adjustment to the scaling law that applied to a much broader range of aftershock sequence data, termed the Omori-Utsu formula or the modified Omori law

$$
\lambda(t)=\frac{K}{(t+c)^{p}}
$$

In Equation [2.9], $K$ is the so-called "aftershock productivity", $c$ is the temporal offset between the mainshock and start of decay and $p$ controls the observed power law-based rate of aftershock decay. Values of $p$ are known to vary regionally, typically between 0.8-1.3 (Ogata, 1989; Shcherbakov et al., 2004, 2005a, 2005b, 2006, 2012, 2013; Nanjo et al., 2007; Bhattacharya et al., 2011). It is evident that as $p$ increases, the sequence decays more rapidly.

This hyperbolic aftershock rate has been observed for a range of large magnitude sequences and holds approximately true even decades after a mainshock (Ogata \& Shimazaki, 1984). However, Ogata (1989) inferred that each subsequent aftershock also has the potential to trigger its own sequence according to its magnitude, and each of the events within could do so as well, forming hierarchical cascades within the larger system. It is possible that this temporal clustering within the occurrence rate becomes even more apparent when modeling smaller events (Ogata \& Zhuang, 2006). Therefore, a more accurate representation of the overall event frequency, in a given region and over a certain time period (or "target window"), would be a superposition of the Omori-Utsu functions of each earthquake plus a constant rate $\mu$ representing background seismicity. According to the ETAS model, each considered seismic event (above the determined completeness magnitude $M_{c}$ ) has the potential to trigger offspring according to the generalized conditional intensity function

$$
\lambda\left(t \mid H_{t}\right)=\mu+\sum_{t_{i}<t} \frac{K e^{\alpha\left(M_{i}-M_{c}\right)}}{\left(t-t_{i}+c\right)^{p}}
$$


where $H_{t}$ is the conditional information of earthquake occurrence history before time $t$ within the target window. Therefore, the rate is modeled as a function of previous activity where, at time $t$, it is conditioned by all events $M_{i} \geq M_{c}$ that satisfy $t_{s} \leq t_{i}<t$, where $t_{s}$ is the start of the window. The parameter $\alpha$ governs the degree of aftershock cascading for a given magnitude. Larger values of $\alpha$ imply a greater sensitivity to magnitude in the generation of aftershocks, which has been observed for great earthquakes (Ogata, 1992; Omi et al., 2014). Conversely, smaller $\alpha$ values reduce the weighting of event magnitude on aftershock triggering. This characteristic has been linked to swarm sequences where mainshocks are less distinct (Mogi, 1963; Utsu, 1970; Ogata, 1988). Estimation of the set of parameters $\varphi=$ $\{\mu, K, \alpha, c, p\}$ fitted to an earthquake dataset may be obtained by maximizing the log-likelihood function for $\lambda(t)$ (see Ogata, 1989).

The quality of the ETAS model fit is generally evaluated based on a transformation of occurrence times. The transformation defines a new set of event-times $\tau_{i}$ as the cumulative conditional intensity function at times $t_{i}$

$$
\tau_{i}=\int_{0}^{t_{i}} \lambda(t) d t
$$

Therefore, a plot of the cumulative ETAS model rate versus the transformed time $\tau$ results in an increasing function with constant unit slope. If the model has been optimized well for the dataset, i.e. events are occurring close to when the model predicts they should, then an overlaid plot of the observed cumulative event count versus the transformed time will match the previous plot with rate close to unity. If the model fits poorly over any stretch of the catalogue, the observed cumulative plot will deviate (positively if the model underestimates events and negatively if the model overestimates) from the constant slope.

Llenos \& Michael (2013) and Lombardi et al. (2010) used a variety of statistical measurements to determine the quality of the model's fit to chosen target windows within a larger catalogue. These measures included the Runs, Auto-Correlation, and Kolmogrov-Smirnov tests on the transformed inter-event times $\Delta \tau_{i}$, as well as the Akaike Information Criterion on the likelihood $L$. They used this approach in order to evaluate the most significant change-point in the seismic rate over time. In Chapter 4, the aim was instead to achieve a fitting of the model to different 
clusters and compare their quality-of-fit (QOF) and parameters, rather than attempt to determine the best fit to a particular data set by varying the target window or other constraints. An alternative, more rudimentary method was therefore applied here to evaluate the QOF, by quantifying the deviations of the observed cumulative plot in transformed time from the ideal constant increasing function. This was achieved by first normalizing the plot so both axes vary from 0 to 1, then computing the area $A$ present between the diagonal (cumulative ETAS) and the observed data. The maximum value of $A$ is 0.5 (half the area of the unit square), corresponding to the worst possible fit to the sequence, whereas a value closer to 0 implies a better fit.

A fitting was attained for each event cluster (the ETAS model is sensitive to the completeness level, and so only events greater than or equal to the estimated $M_{c}$ were used), and the absolute parameter values and goodness-of-fit were assessed. While it is acknowledged that ETAS parameters for natural seismicity are known to vary regionally (the $\mu$ and $p$ values in particular), all the sequences in question occurred within regional vicinity of one another and appear to have been triggered artificially. It is therefore proposed here that the differences or similarities observed in cluster parameters may be at least partially influenced by their causal mechanisms.

\subsection{The Nearest-Neighbour Distance (NND) Model}

The NND model is a statistical approach to earthquake cluster identification and classification, first formulated by Baiesi \& Paczuski (2004) and expanded significantly by Zaliapin et al. (2008) and Zaliapin \& Ben-Zion (2013a, 2013b, 2016). Its purpose is to link together and characterize event families or clusters using a rescaled inter-event distance metric termed the nearest-neighbour distance $\eta$, which is defined below as space, time and magnitude dependent. This method was applied in Chapters 3 and 4 in order to describe both the regional and local inter-event distance distributions within the WCSB, as well as to statistically categorize the types of seismic clustering observed. 
Construction and evaluation of the NND model are achieved through the following steps:

a) Calculate inter-event distance values $\eta_{i j}$ between all possible pairs of events $(i, j)$ within a catalogue above a certain magnitude threshold.

b) Assign the smallest observed distance value $\eta_{j}=\min _{i} \eta_{i j}$ to each event $j$ and assign the event $i$ corresponding to this minimum distance as the potential parent of offspring event $j$. The term nearest-neighbour is used interchangeably between parent and offspring events, as in "events $i$ and $j$ are nearest-neighbours".

c) Plot the histogram of $\log _{10} \eta$ values to observe and quantify modality in the distribution within the framework of a Gaussian mixture model (discussed below). Hicks (2011) showed that bimodality in $\eta$, i.e. a distinction between background and clustered events, is an intrinsic property of both worldwide and regional seismicity.

d) Define this distinction as a constant threshold value $\log _{10} \eta_{\text {thresh }}$ between the subpopulations such that earthquakes whose inter-event distances $\log _{10} \eta<$ $\log _{10} \eta_{\text {thresh }}$ are classified as strongly linked to their parent event (within the clustered mode) and otherwise are classified as weakly linked (within the background mode).

e) Construct a spanning network, or spanning forest, by linking together temporal hierarchies of strongly linked parents and offspring (nearest-neighbours) as individual sequences or "event families". Each event family is a tree in the spanning forest and may be represented in topological space as a directed graph without loops or cycles. Events that are only weakly linked to both parents and potential offspring are categorized as their own sequence and termed singles. In natural tectonic settings, singles tend to make up the majority of the spanning forest.

f) Classify the individual cluster trees as aftershock sequences, seismic swarms, a combination of the two or neither based on their structure and topology using statistical parameters suggested by Zaliapin \& Ben-Zion (2013b) (examples are provided in the following subsections). 


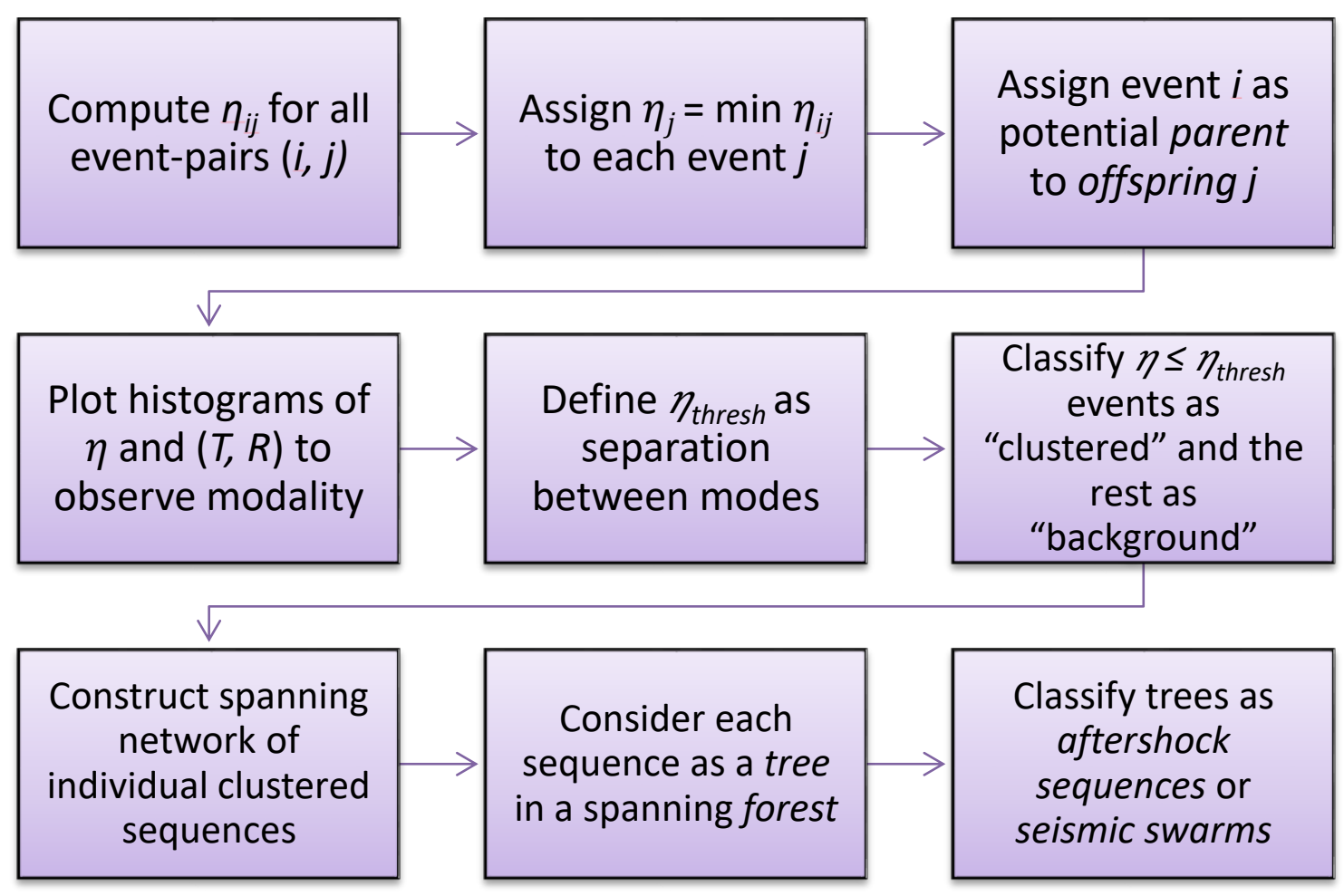

Figure 2-5: Simplified description of the NND method workflow.

\subsubsection{The Rescaled Inter-Event Distance Metric $\eta$}

The distance values $\eta_{i j}$ are defined based on the spatiotemporal distance between each event pair within the catalogue as well as on the magnitude of the event that occurred first (the potential parent event $i$ ). Specifically, each event $j$ is assigned values $\eta_{i j}$ based on its relationship with all other events $i$ as follows

$$
\eta_{i j}=\left\{\begin{array}{c}
t_{i j}\left(r_{i j}\right)^{d_{f}} 10^{-b m_{i}}, t_{i j}>0 \\
\infty, \quad t_{i j} \leq 0
\end{array} .\right.
$$

Here $t_{i j}=t_{j}-t_{i}$, which is the time in days between event $j$ and event $i$. Note that event $j$ must succeed event $i$ in order for the quantity $t_{i j}$ to be positive, otherwise $\eta_{i j}=\infty$. Clearly, the quantity $\eta_{i j}$ is asymmetric in time. This implies that event-family trees are structured chronologically top-down without loops or cycles (see Chapter 2.5.3 for a brief discussion on 
topology). Therefore, each parent event may have multiple offspring, but each offspring event may have only one parent.

The inter-event spatial distance $r_{i j}$ is computed between epicenters using the Haversine formula for great-circle distance (or arc length) in kilometers

$$
r_{i j}=2 r_{e} \arcsin \sqrt{\sin ^{2} \frac{\left(\varphi_{i}-\varphi_{j}\right)}{2}+\cos \varphi_{i} \cos \varphi_{j} \sin ^{2} \frac{\left(\lambda_{i}-\lambda_{j}\right)}{2}}
$$

In Equation [2.13], $r_{e}$ is the Earth's radius estimated as $6378.14 \mathrm{~km}$, and $\varphi_{i, j}$ and $\lambda_{i, j}$ are the latitudinal and longitudinal coordinates of events $i$ and $j$, respectively.

$d_{f}$ is the fractal spatial dimension of earthquake location distribution. In 2 dimensions, for both local and worldwide epicentral distributions, $d_{f}$ has been found to vary approximately between 1.2 and 1.6 (Sadovsky et al., 1984; Kagan, 1991; Kosobokov \& Mazhkenov, 2013). $m_{i}$ is the magnitude of the $i^{\text {th }}$ event and $b$ is the estimated GR $b$-value of the dataset.

The quantities $b$ and $d_{f}$ are often assumed constant when applying the NND method regionally over time, partly because regional parameter estimations tend to introduce some bias and partly to constrain the variability of results to the three core variables of inter-event distance $r$, time $t$ and magnitude $m$ (Zaliapin \& Ben-Zion, 2013a; Schoenball et al., 2015; Vasylkivska \& Huerta, 2017). Zaliapin \& Ben-Zion (2013a) also demonstrate that the algorithm is stable with respect to the chosen catalogue cut-off magnitude $M_{0}$, with the biggest trade-off being the proportion of background events to clustered events. As $M_{0}$ increases, more events $m_{i}<M_{0}$ are removed from clusters until some are eventually reduced to singles. Here, the methodology of other studies was followed by maintaining a lower magnitude cut-off to maximize sampling and fixing $d_{f}=1.5$. Furthermore, the GR $b$-value was fixed as $b=1.0$ in the regional analysis (Chapter 3), but actual $b$-value estimates were utilized when analysing specific clusters (Chapter 4). 
The scalar distance $\eta_{i j}$ may be expressed in terms of its rescaled temporal and spatial components by defining

$$
\begin{gathered}
T_{i j}=t_{i j} 10^{-\frac{b m_{i}}{2}} \\
R_{i j}=r_{i j} d_{f} 10^{-\frac{b m_{i}}{2}} .
\end{gathered}
$$

By this formulation, $\eta_{i j}=T_{i j} R_{i j}$. Once nearest-neighbour values $\eta$ have been determined for each event $j$, where $\eta_{j}=\min _{i<j} \eta_{i j}$, the joint distribution of $(T, R)$ as well as their individual histograms are plotted to observe possible modality in temporal and/or spatial distance between clustered and background events.

\subsubsection{Formal Analysis of Modality in the $\eta$ Distribution}

Figure 2-6 shows an example of the clustered and background earthquake subpopulations present within the NND distributions of southern California. 
(a)

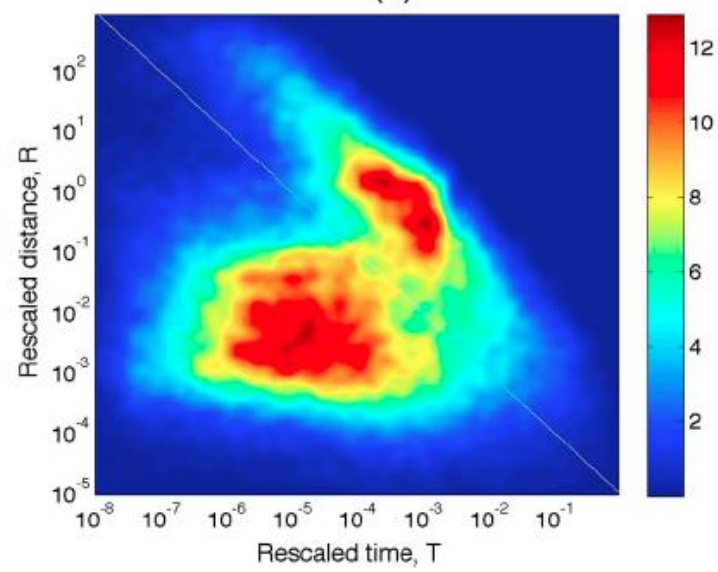

(c)

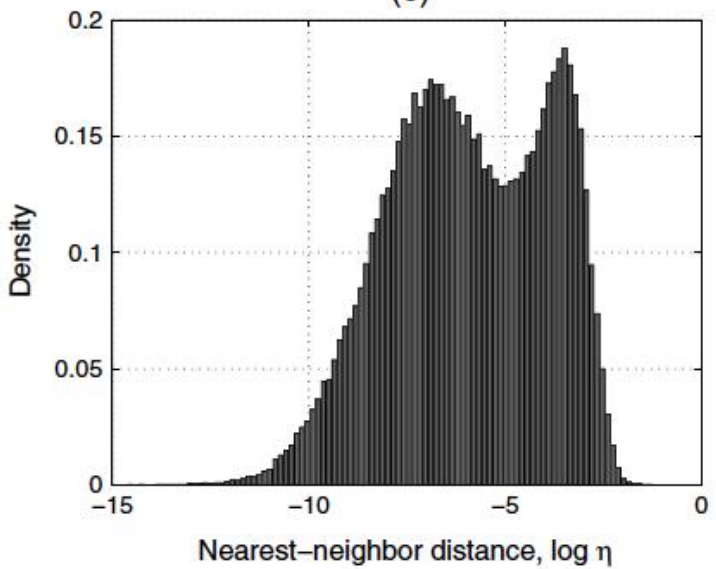

(b)

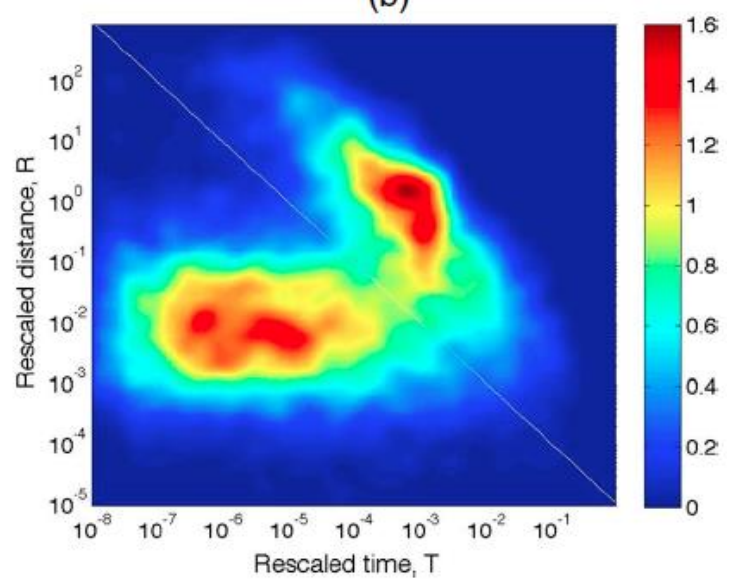

(d)

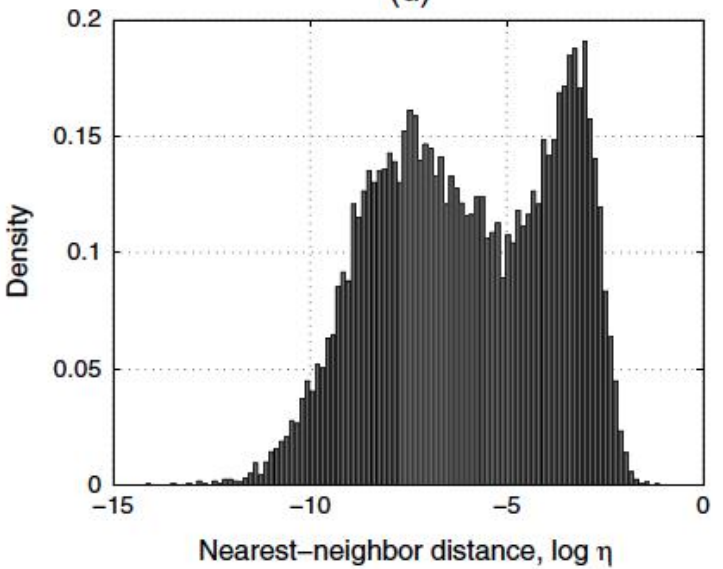

Figure 2-6: NND distributions of southern California earthquakes for different cut-off magnitudes. (a, b) The joint distributions of the temporal $(T)$ and spatial $(R)$ components; diagonal line represents the separation between the two modes. (c, d) The normalized densities of $\eta$ values; modal separation occurs at $\log _{10} \eta_{\text {thresh }} \approx-5$. (a, c) $M_{0}=2$; (b,d) $M_{0}=3$. (From Zaliapin \& Ben-Zion, 2013a).

Two modes are observed in each plot. One appears at small values of $\eta$, corresponding to small values of both $T$ and $R$, implying that events are occurring closely together - this is the clustered mode. The other mode occurs at larger values of $\eta$, corresponding to larger values of $T$ and $R$, implying events are occurring further apart in space and time - this is the background mode. Note that the distributions are stable with respect to $M_{0}$; the modal separation point remains independent of the cut-off magnitude (Zaliapin \& Ben-Zion, 2013a). 
Although the subpopulations may be obvious upon visual inspection, it is useful to define them rigorously considering a Gaussian mixture model (GMM), as detailed in Hicks (2011). A GMM is defined as a composition of normal density functions, each with a mean, covariance and mixing proportion (or weight). The parameters for these component functions are estimated using the 2-step Expectation-Maximization (EM) algorithm (Hastie et al., 2009). The approach uses an initial guess for the set of parameters to then:

a) Calculate Bayesian probabilities for each data point as a possible member of each mode. This is the Expectation Step.

b) Estimate the model parameters for each mode through their maximum likelihood function, using the probabilities determined in the Expectation step as weights. This is the Maximization Step.

This process is iterated until it converges to the optimal estimation of the means, standard deviations and weights. The number of modes in the distribution is estimated using information criteria. Two such criteria were used in this study, the Akaike Information Criterion (AIC) and Bayesian Information Criterion (BIC) (Akaike, 1974; Schwarz, 1978). The AIC and BIC evaluate a model's fit to a dataset by rewarding its likelihood $L$ and penalizing complexity via its number of parameters $k$ as well as its variance within the sample size $n$ as

$$
\begin{gathered}
A I C=-2 \log L+2 k ; \\
B I C=-2 \log L+k \log n .
\end{gathered}
$$

The model that minimizes the information criteria is considered the best fit. The threshold value $\log _{10} \eta_{\text {thresh }}$, which separates the clustered mode from the background, is chosen as the intersection point between the resulting component densities. Note that the constant value of $\log _{10} \eta_{\text {thresh }}$ is represented as a vertical separation in the one-dimensional $\log _{10} \eta$ distributions and as a downward diagonal, $\log _{10} \eta_{\text {thresh }}=\log _{10} T-\log _{10} R$, in the joint distributions (Figure 2-6). 


\subsubsection{Classification of Event Clusters}

Once the $\log _{10} \eta_{\text {thresh }}$ value has been determined and the event catalogue has been divided into the clustered (events whose $\log _{10} \eta_{j}<\log _{10} \eta_{\text {thresh }}$ ) and background (all remaining events) domains, the data can be further discretized into hierarchical event families based on the strong links between parents and offspring. The largest event in each family is classified as the mainshock; if there is more than one largest-magnitude event then the first is considered the mainshock. Events in the sequence that occur before the mainshock are called foreshocks and occur after are called aftershocks. Earthquakes that are not strongly linked to any other event are considered their own cluster and classified as singles (Figure 2-7). This methodology implies two things: firstly, that all single events are background events, and secondly, that the first event or "oldest ancestor" in each sequence is also a background event. The second implication resembles the underlying seeding mechanism of the stochastic ETAS model, which requires a nonzero background rate $\mu$ from which to generate aftershocks.

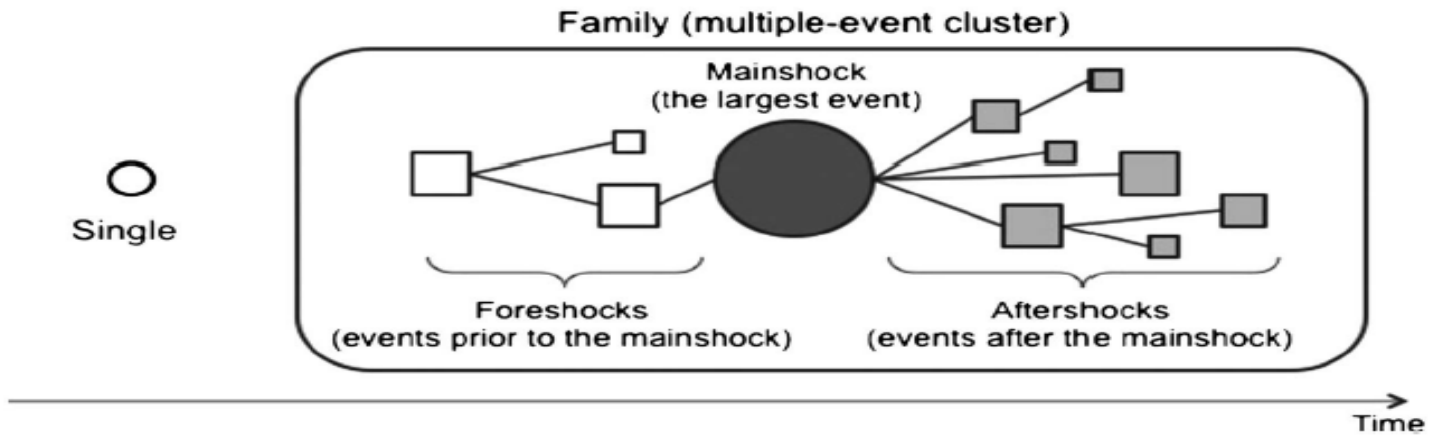

Figure 2-7: Event classification methodology. Left-hand side: a single event (white circle), characterized by only weak links to both potential parent and offspring events. Righthand side: an event family connected by strong links. The dark circle is the largest event and is labeled the mainshock; it is preceded in time by smaller foreshocks (white squares) and succeeded by smaller aftershocks (grey squares). (From Zaliapin \& Ben-Zion, 2013a).

Once event families have been identified, they can then be characterized as aftershock sequences, seismic swarms, a combination of the two or neither. To do so, each cluster is represented as a time-magnitude sequence, a spatial map and a time-oriented tree graph. Figure 2-8 and Figure 2-9 show two sample clusters displayed in this manner. Cluster 1 is a typical aftershock or burst sequence and Cluster 2 is a swarm. 
a)

$$
t_{D}=287.1
$$

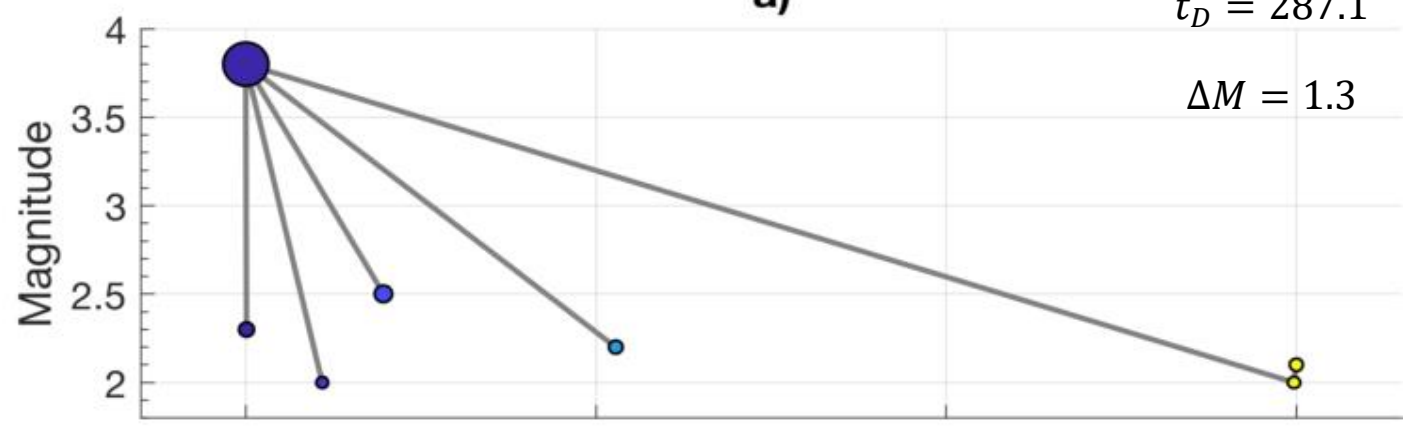

$$
19 / 11 / 84 \quad 16 / 12 / 84
$$

$12 / 01 / 85$

$09 / 02 / 85$

Time

b)

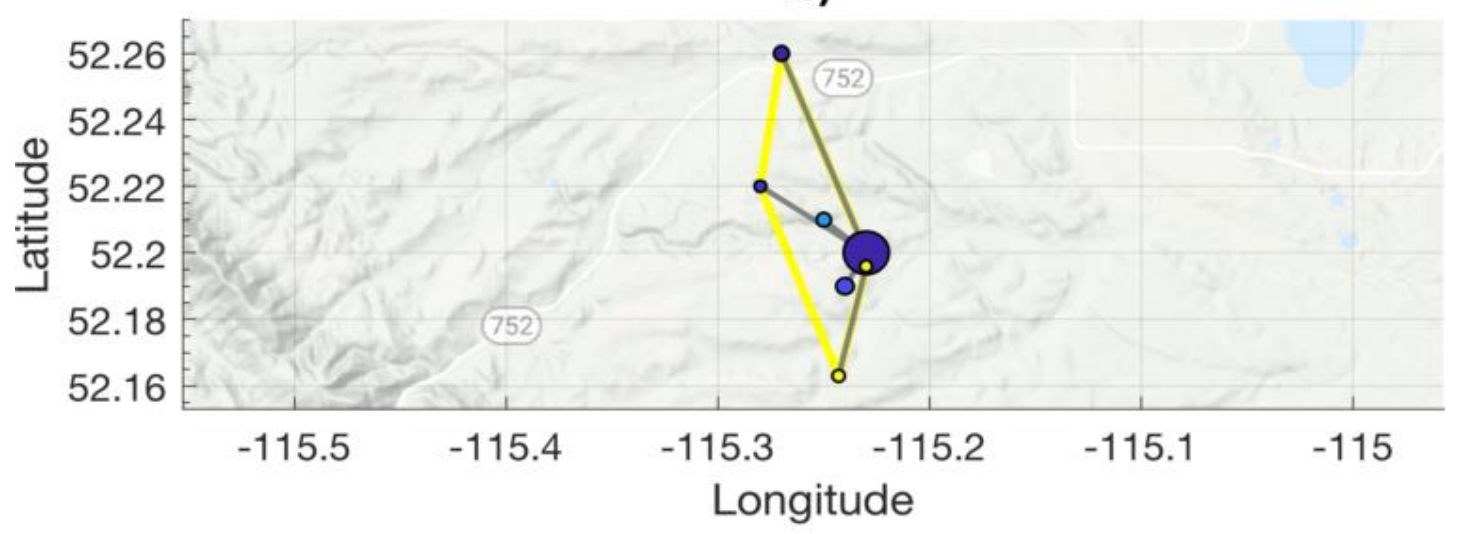

c)

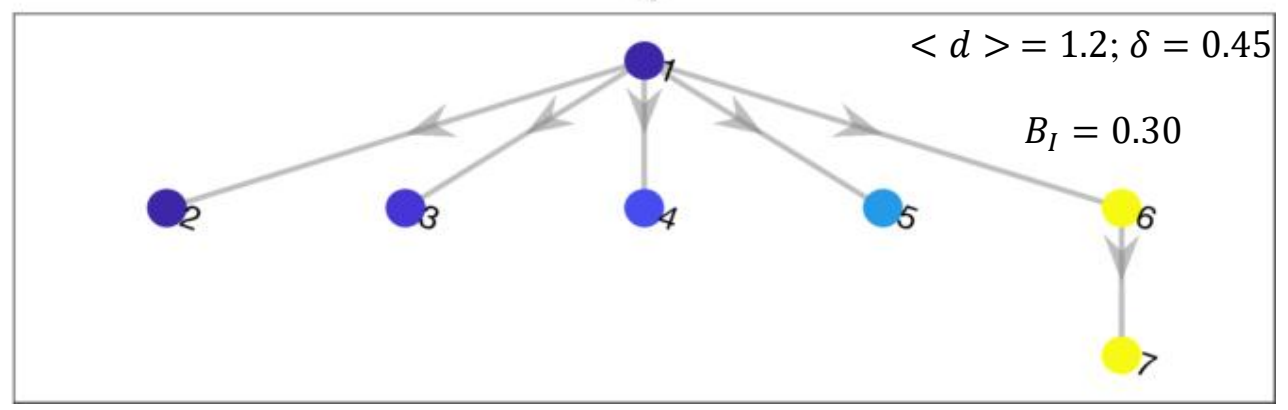

Figure 2-8: Cluster 1: characterization of an example "burst" sequence. Circles represent earthquakes and are coloured chronologically from darkest to lightest; grey lines represent the strong links connecting them. a) Event family magnitudes vs. times of occurrence in days. b) Spatial map of events; yellow border outlines the hull area occupied by the sequence. c) The topological family tree, which branches downward in time. 
a)

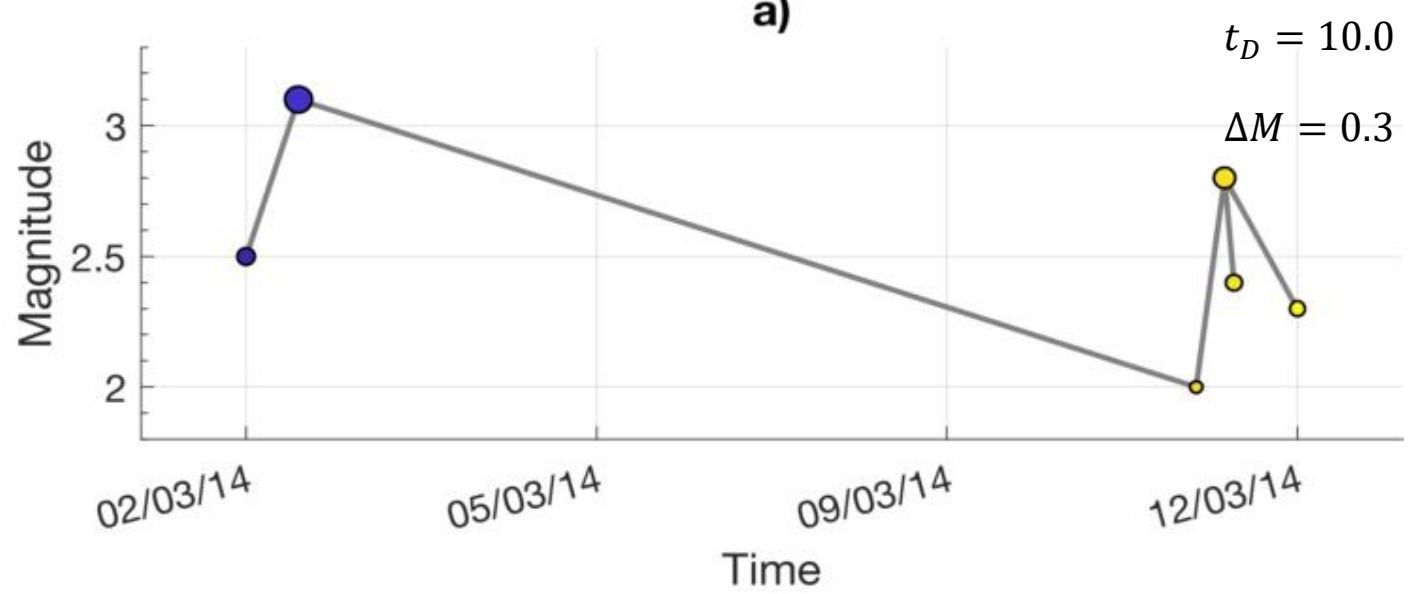

b)

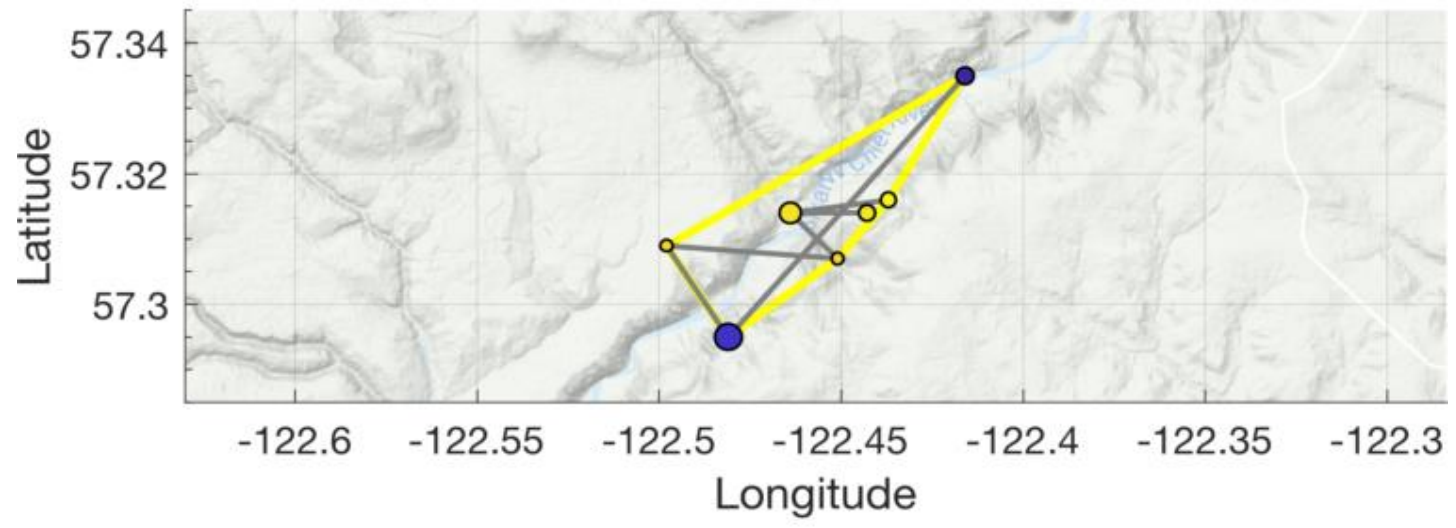

c)

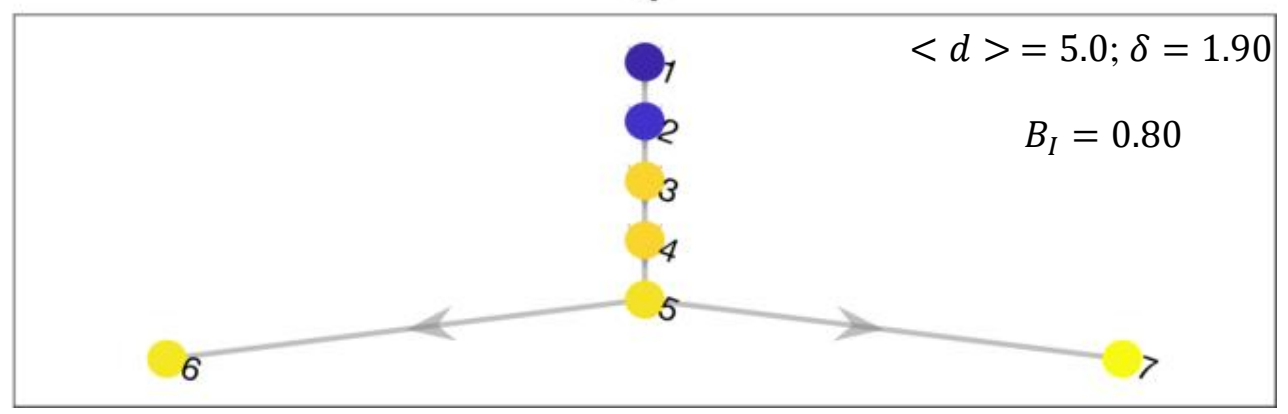

Figure 2-9: Cluster 2: characterization of an example "swarm" sequence. Circles represent earthquakes and are coloured chronologically from darkest to lightest; grey lines represent the strong links connecting them. a) Event family magnitudes vs. times of occurrence in days. b) Spatial map of events; yellow border outlines the hull area occupied by the sequence. c) The topological family tree, which branches downward in time. 
Clearly, the shaping of the cluster structures differs significantly. In all three panels, it is intuitively seen that Cluster 1 displays a spray or burst-like shape, characteristic of aftershock sequences occurring due to sudden, brittle failure. These types of clusters typically involve a dominant mainshock causing many low-magnitude, $1^{\text {st }}$ generation offspring in multiple directions and fewer foreshocks or higher order offspring. On the other hand, Cluster 2 has a linear or path-like shape where events are chained together gradually, characteristic of seismic swarms occurring along fault channels in more ductile rheology. In these cases, the mainshock is less distinct (the magnitude differential between the largest and second largest event in the sequence is smaller), and is often preceded by foreshocks and succeeded by multiple generations of offspring. Zaliapin \& Ben-Zion (2013b) demonstrate that these distinguishable characteristics of seismic clustering appear throughout their analyses of southern California earthquakes, scaling upwards to M6+ sequences containing hundreds of events. They find swarm and burst-like event families can differ in up to a dozen parameters relating to their temporal, spatial and topological structures, and occur predominantly in different geologic settings. As mentioned, swarms tend to appear in more ductile areas of higher heat and/or fluid flow, whereas burst sequences occur in cooler, more brittle regions with lower levels of fluid flow.

While visual inspection is useful to illustrate the general methodology applied when distinguishing aftershock sequences and swarms in the above manner, it is neither efficient nor rigorous enough for a formal analysis of a full catalogue of events. Zaliapin \& Ben-Zion (2013b) therefore introduced statistical parameters to quantify the branching characteristics of event families and determine their structure type. Several of these measures were adopted in the cluster analysis in Chapter 4, which utilize basic concepts from graph theory.

A graph is composed of nodes and edges. Nodes represent data as points on the graph and edges represent the relationships between data points as links that connect them. A graph is "directed" if there is an orientation that defines the relationship between nodes. This is represented by directed edges, which are edges that point explicitly from one node to another. In the case of event cluster construction, each structure is time-oriented where every earthquake is a node connected directly to its nearest-neighbour by an edge, beginning from the first event in the sequence and branching downward chronologically. The definition of the nearest-neighbour 
distance $\eta$ given in Equation [2.12] implicitly states that, not only is $\eta$ asymmetric in time (where $\eta_{i j} \neq \eta_{j i}$ ), but necessarily that if $\eta_{i j}<\infty$, then $\eta_{j i}=\infty$. In other words, the only possible direct relationship between nodes is a parent-to-child relation. Therefore, no loops (directed edges pointing in both directions) or cycles (directed paths that eventually end where they begin) can exist within the structure.

With the above criteria established, the terminology of a rooted tree-graph may be employed, which considers the first event in a sequence as the "root" of the tree, the downward-directed edges as "branches" and the end-nodes (earthquakes that have no offspring) as "leaves". The size $N$ of a sequence is equal to its topological order, which is computed simply as the total number of nodes. The leaf depth $d$ is calculated by counting the number of branches connecting each leaf back to its root. The average leaf depth $\langle d\rangle$ of a tree provides an indication of its shape, with larger $\langle d\rangle$ potentially indicating higher levels of event chaining and a deeper structure, and smaller $\langle d\rangle$ implying low orders of event offspring and a shallower structure. Therefore, swarm sequences are expected to have larger $\langle d\rangle$ values than bursts, even given a similar number of leaves. This can be seen clearly in the two graphs shown in the third panels of Figure 2-8 and Figure 2-9 (panels c); Cluster 1 has a $\langle d\rangle$ value of 1.2 while Cluster 2 has a $\langle d\rangle$ value of 5.0. However, since $\langle d\rangle$ also scales with the sequence size $N$, a normalized leaf depth $\delta$ can be useful on a broader scale, which is defined as

$$
\delta=\frac{\langle d\rangle}{\sqrt{N}}
$$

The inverted branching number $B_{I}$ of a tree is computed as the number of parent events (events that have at least one offspring) divided by the total number of branches. An acyclic rooted-tree graph with no loops contains $N-1$ branches, where $N$ is the number of nodes. Therefore a maximum $B_{I}$ value of unity indicates a perfect path shape and only a single leaf within the structure. Smaller values of $B_{I}$ imply more offspring from fewer parents, i.e. a more burst-like formation. In the sample clusters shown by Figure 2-8 and Figure 2-9, Cluster 1 has a $B_{I}$ value of 0.3 while Cluster 2 has a $B_{I}$ value of 0.8 .

Non-topological parameters can also be indicative of sequence type, namely the magnitude differential $\Delta m$ between the designated mainshock and second-largest event (foreshock or 
aftershock), as well as the spatial area $A$ and decay period $t_{D}$ covered by the sequence. $A$ is computed in $\mathrm{km}^{2}$ as the convex hull area containing all events (yellow border, panels $\mathrm{b}$ in Figure 2-8 and Figure 2-9), and $t_{D}$ is measured in days as the time elapsed between the occurrence of the root event and the deepest leaf. Zaliapin \& Ben-Zion (2013b) showed that topologically shallower structures tend to have larger $\Delta m$ and smaller $A$ and $t_{D}$ values than do deeper structures, given a similar order $N$. However, this is not always the case, and since these non-topological parameters also scale with $N$ they cannot be relied upon solely. Overall, smaller $\langle d\rangle, \delta, B_{I}, A, t_{D}$, and larger $\Delta m$ values typically correlate with the aftershock sequence, where the mainshock is sudden and dominant, creating a spray of rapidly decaying, first-order offspring in many directions whose magnitudes are usually not large enough to generate further significant clustering. Conversely, larger $\langle d\rangle, \delta, B_{I}, A, t_{D}$ and smaller $\Delta m$ values correlate with the archetypal swarm, where events are often of comparable size and tend to "travel" gradually along fault channels away from the initial event.

The parameter sets $\theta=\left\{\langle d\rangle, \delta, B, A, t_{D}, \Delta m\right\}$ for all significant event families within each examined cluster along the WCSB were determined in Chapter 4. The first three (topological) parameters in $\theta$ were primarily used for classification of the sequence type. If they proved inconclusive, the last three parameters were taken into consideration as well. 


\section{Chapter 3}

\section{Regional Analysis of Nearest-Neighbour Distance Distributions within the Western Canada Sedimentary Basin}

In this chapter, the results of the Nearest-Neighbour Distance (NND) model application to the regional Composite Alberta Seismicity Catalogue (CASC; detailed in Chapter 2.1) are presented, where both the entire time period (1975-2018) and separated time windows (19752009, 2010-2018) were analyzed. The separation point between time windows was chosen to correspond with the rapid rise in HF implementation within the region (e.g. Atkinson et al., 2016).

The spatial boundaries of the study region encompass a rectangular area spanning $\left[49^{\circ}-60^{\circ} \mathrm{N}\right.$ ] latitude and $\left[110^{\circ}-125^{\circ} \mathrm{W}\right]$ longitude, including all of Alberta and a portion of eastern British Columbia (Figure 3-1). Zaliapin \& Ben-Zion (2013a, 2013b) showed that NND distributions are stable for cut-off magnitudes less than the regional completeness magnitude $\left(M_{c} \approx 3.0\right.$ in this case, see Adams \& Halchuk (2003) and Cui et al. (2015)), and therefore a magnitude cutoff of $M_{0}=2.0$ is applied to enrich sampling. Furthermore, a sensitivity analysis for the present dataset is provided in the appendices, which shows that the results derived in this chapter hold for cut-off magnitudes up to the regional completeness level (Appendices A \& B). The same constant values $d_{f}=1.5$ and $b=1.0$ are used for each subset of the regional data to illustrate the fundamental differences observed over time even without prior parameterization. 


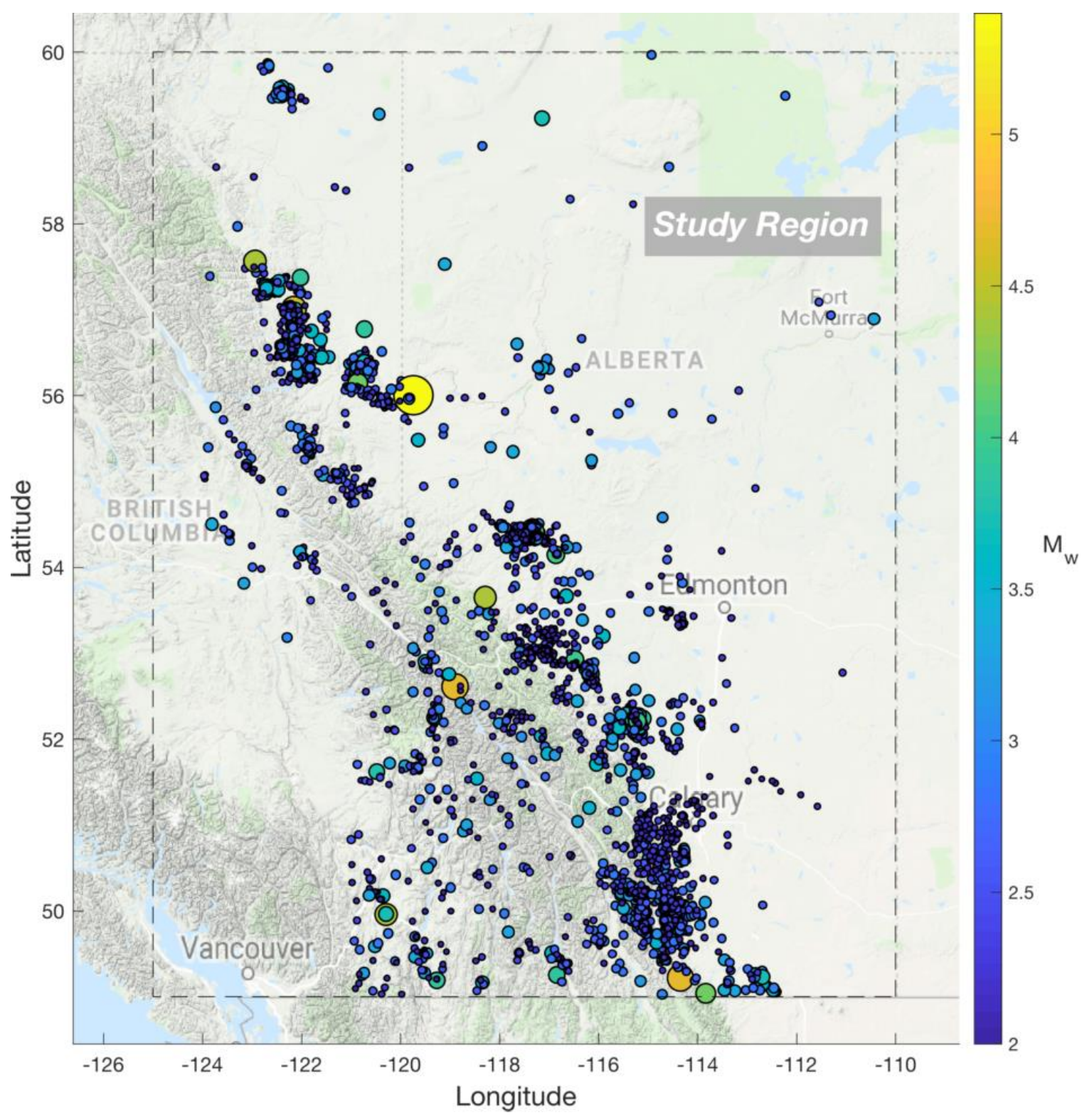

Figure 3-1: Map of study region. Markers are $M \geq 2$ seismic events between 1975-2018; size and colour indicate earthquake magnitude.

\subsection{Entire Time Period (1975-2018)}

Figure 3-2 shows the 2-dimensional $(T, R)$ distribution and the normalized density of nearestneighbour distances $\eta$ for the entire study period. There appear to be two prevailing modes within the joint distribution (panel a), which differ in size and shape. The clustered mode, to the bottom-left of the plot beneath the bold white line, is more concentrated and oriented somewhat horizontally, while the background mode, in between the two white lines, covers a broader 
range along the $T$ and $R$ axes and is faintly oriented along the downward diagonal. This observation is consistent with findings in other studies, which showed the existence and distinct shaping of clustered and background earthquake subpopulations in several tectonic and/or induced areas (Hicks, 2011; Zaliapin \& Ben-Zion, 2013a, 2016; Schoenball et al., 2015). Notably, in all cases the background mode was reminiscent of a time-stationary, spaceinhomogeneous marked Poisson (SIP) process, which forms a unimodal distribution concentrated along a downward diagonal at large $T$ and $R$, while the clustered mode typically occupies a horizontally oriented ellipse with $T$ and $R$ values much smaller than would be expected from a Poisson process (Zaliapin \& Ben-Zion, 2013a). The chief disparity between different regions, including within the WCSB, lies in the relative intensities or "mixing proportions" of each mode; these proportions tend to reflect the nature of seismicity occurring (discussed further below). More difficult to see in Figure 3-2 is a third subpopulation, to the upper-right above the thin white line, which is much less concentrated and sprawls along a downward diagonal over large values of $T$ and $R$. The one-dimensional $\eta$ distribution (panel b) reflects this tri-modality clearly, with the third mode at large $\eta$ perhaps more apparent. 
a)

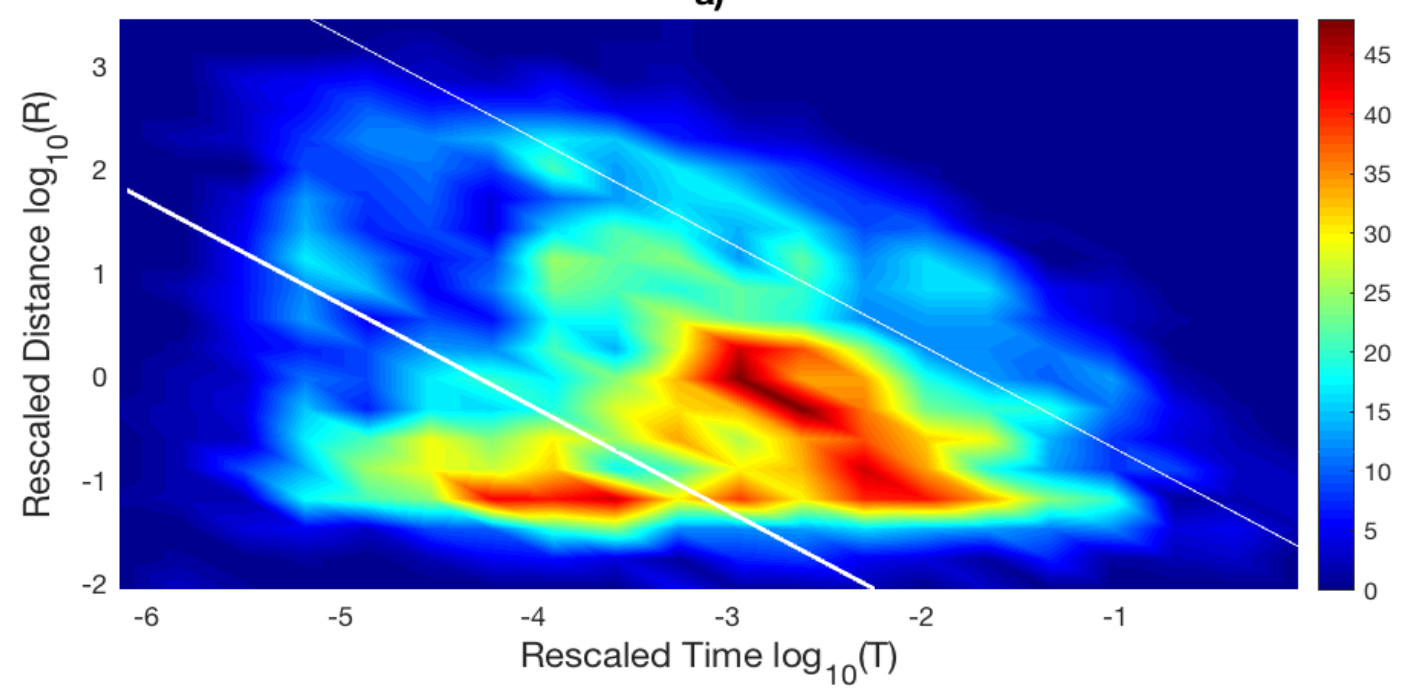

b)

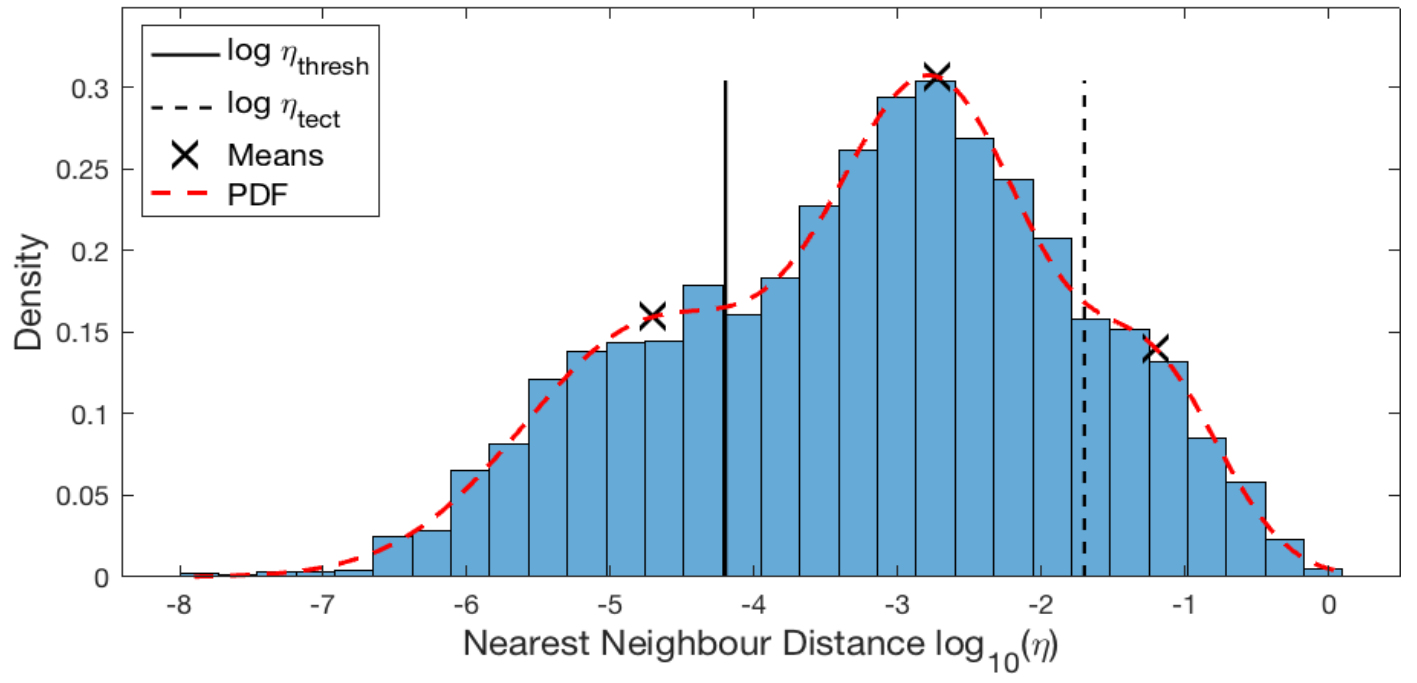

Figure 3-2: NND distribution of the regional WCSB catalogue (1975-2018) using $M_{0}=$ 2. 0. a) Joint distribution of the temporal and spatial components $(T, R)$. Bold white line indicates the threshold $\log _{10} \eta_{\text {thresh }}$ between tightly clustered and loosely clustered components. Thin white line indicates the threshold $\log _{10} \eta_{t e c t}$ between loosely clustered and deep-background components. Colour bar indicates frequency of inter-event distance occurrence. b) Normalized density of $\eta$ values. Solid black line is $\log _{10} \eta_{\text {thresh }}$ and dashed black line is $\log _{10} \eta_{\text {tect }}$. Dashed red line is the normalized probability density function of the Gaussian mixture and black crosses are the component means. 
The results of the Gaussian mixture model (GMM; detailed in Chapter 2.5.2) analyses, performed on the CASC dataset from 1975 to 2018 assuming 1, 2, 3 and 4 modes, are presented in Table 3-1. They indicate that the optimal mixture estimation, i.e. a minimization of the AIC and BIC, is a 3-component GMM instead of the typically observed 2-component (clustered and background) distribution. This can be observed in Figure 3-2 panel b, where the dashed red line and black crosses represent the resulting probability density function and 3 component means, respectively.

Table 3-1: Expectation Maximization results for the GMM applied to the regional WCSB catalogue (1975-2018) using $M_{0}=2.0 . k$ is the number of modes, AIC and BIC are the resulting Akaike and Bayes Information Criteria. Highlights show the minimized values.

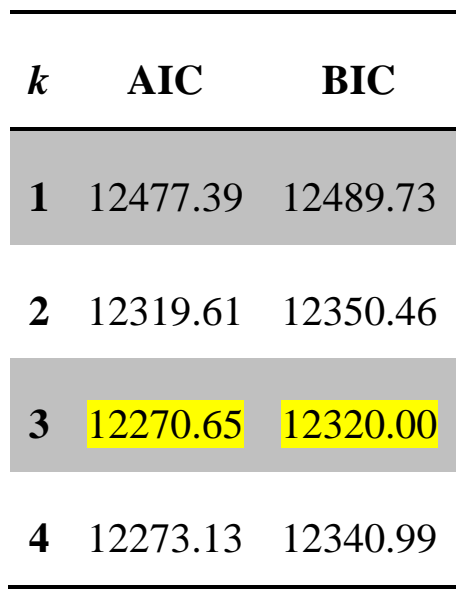

The intersection point between the two dominant modes occurs at $\log _{10} \eta_{\text {thresh }} \approx-4.2$, shown by the bolded white line in Figure 3-2 panel a, and solid black line in panel b. Table 3-2 provides the classification and mixing proportions of the inter-event distribution. Out of the 3531 total events analyzed, 29\% were found in the clustered domain, which is henceforth referred to as the tightly clustered mode. $56 \%$ were located in the middle mode, which is henceforth referred to as the loosely clustered mode, and $15 \%$ were located in the third mode, henceforth referred to as the deep-background. The intersection between the two background modes occurs at $\log _{10} \eta_{\text {tect }} \approx-1.7$, shown by the thin white line in Figure 3-2 panel a, and dashed black line in panel $b$. 
Table 3-2: Gaussian mixture proportions for the regional WCSB catalogue (1975-2018) using $M_{0}=2.0$.

\begin{tabular}{cccc}
\hline $\mathbf{N}$ & $\begin{array}{c}\text { Tightly Clustered }(< \\
\left.\log _{\mathbf{1 0}} \boldsymbol{\eta}_{\text {thresh }}\right)\end{array}$ & $\begin{array}{c}\text { Loosely Clustered }(\geq \\
\left.\log _{\mathbf{1 0}} \boldsymbol{\eta}_{\text {thresh }}\right)\end{array}$ & $\begin{array}{c}\text { Deep-Background (〉 } \\
\left.\log _{\mathbf{1 0}} \boldsymbol{\eta}_{\text {thresh }}\right)\end{array}$ \\
\hline $\mathbf{3 5 3 1}$ & $1019(28.9 \%)$ & $1973(55.9 \%)$ & $539(15.3 \%)$ \\
\hline
\end{tabular}

Figure 3-3 plots the histograms of the individual rescaled temporal distances $T$ and spatial distances $R$. No definitive trends are apparent in the individual $T$ or $R$ distributions, with the exception of an increasing proportion of small $R$ distances that tend to stack at the limits of network location resolution, giving the tightly clustered mode its azimuthal shape. This agrees with another observation of Zaliapin \& Ben-Zion (2013a), who stated that the modality in $\eta$ cannot be fully explained by marginal trends present in either $T$ or $R$ but is in fact dependent on the association between the two, as seen in the 2-dimensional joint distributions (Figure 3-2, panel a). 
a)

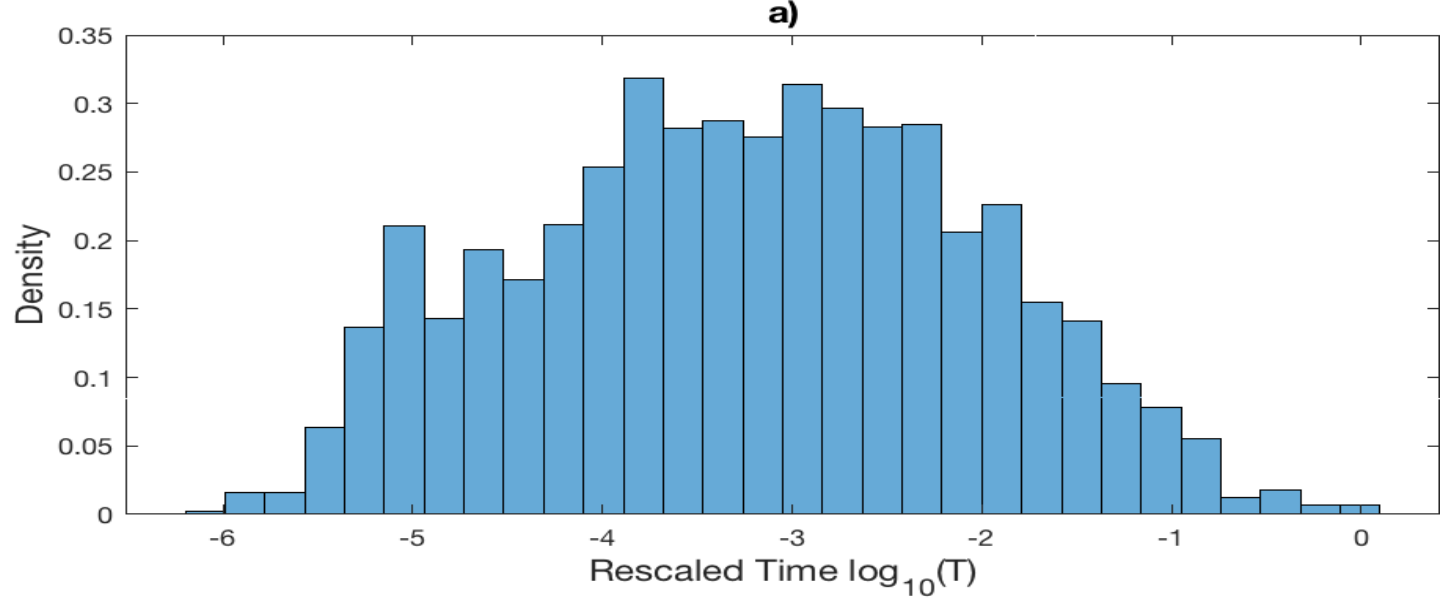

b)

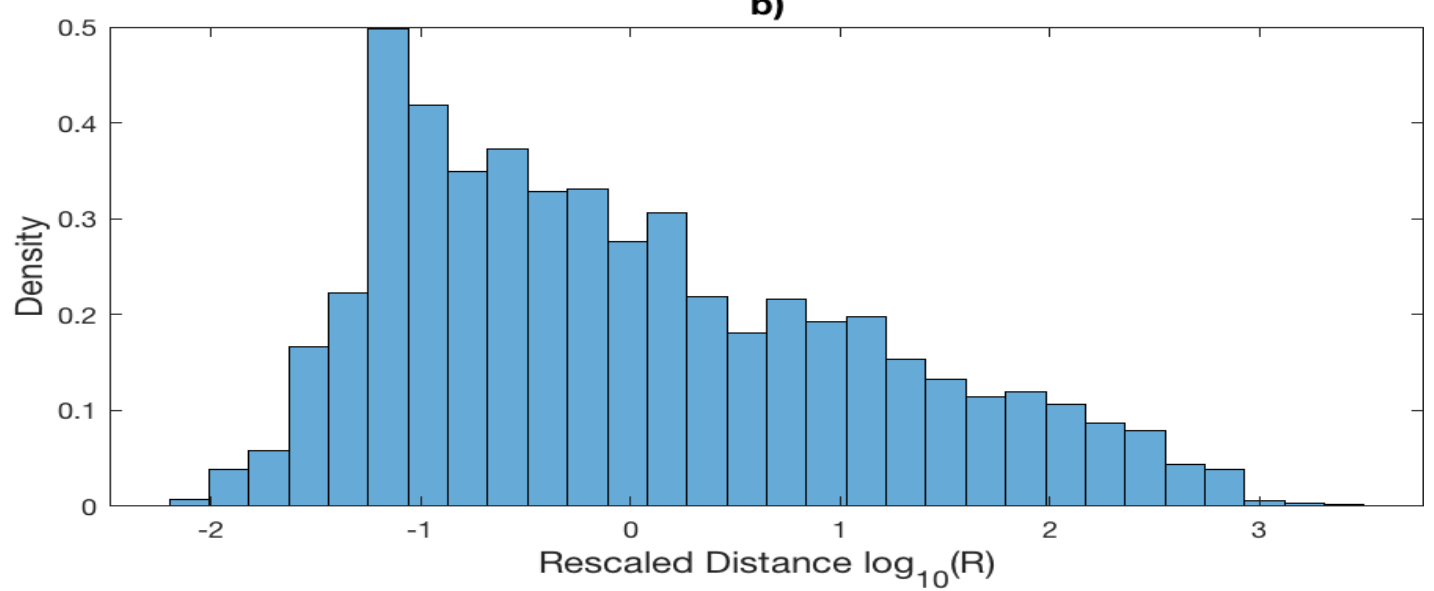

Figure 3-3: Individual histograms of inter-event time $(T)$ and space $(R)$ for the regional WCSB catalogue (1975-2018) using $M_{0}=2.0$. a) Histogram of rescaled times $T$. b) Histogram of rescaled distances $R$.

\subsubsection{Comparative Analysis of Mixture Components}

Figure 3-4 plots the modal decomposition of the joint distribution, where each subpopulation is plotted separately, removing the dependence on mixing proportion (the colour schemes are normalized here to reflect relative inter-event intensity as opposed to raw occurrence frequencies). As mentioned above, if a distribution does not intensify along the bisecting diagonal (constant $\eta$ ) and instead forms an elliptical cloud, then a trend exists in the data as a deviation from Poisson behavior. As expected, the tightly clustered subpopulation deviates substantially from the diagonal and forms an ellipse within the sub-region $\left\{-5<\log _{10} T<-3.5 \mid-1.5<\log _{10} R<-0.5\right\}$. The loosely clustered mode is faintly 
concentrated along the diagonal yet also forms a cloud within the sub-region $\left\{-3.5<\log _{10} T<-1 \mid-1.5<\log _{10} R<1\right\}$. By contrast, the deep-background is distributed very closely along the diagonal and stretches almost its complete length, most clearly resembling a time stationary, space inhomogeneous Poisson (SIP) process.
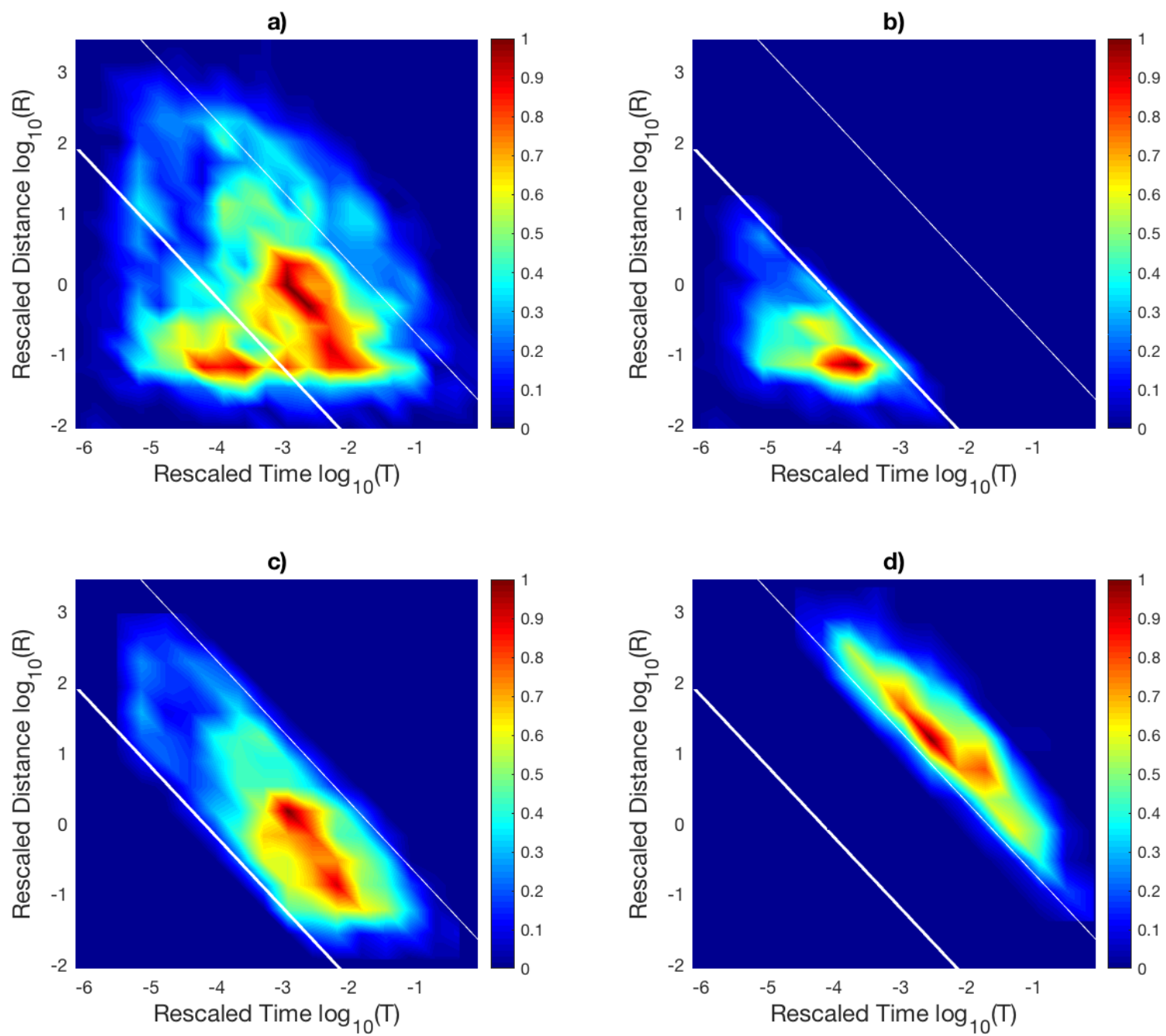

Figure 3-4: Joint $(T, R)$ distributions of individual modes for the regional WCSB catalogue (1975-2018) using $M_{0}=2.0$. Colour bar reflects relative NND intensity. Bold white line indicates the threshold $\log _{10} \eta_{\text {thresh }}$ between tightly clustered and loosely clustered components. Thin white line indicates the threshold $\log _{10} \eta_{\text {tect }}$ between $\operatorname{loosely}$ clustered and deep-background components. a) Entire distribution. b) Tightly clustered mode. c) Loosely clustered mode. d) Deep-background. 
The magnitude scaling of events within each component may provide some insight into their individual characteristics. Figure 3-5 shows the frequency-magnitude distributions (FMDs) for the regional catalogue, as well as for the determined NND subpopulations. The GutenbergRichter (GR) $b$-values are provided for each distribution, calculated using the regional estimated completeness value $M_{c}=3.0$ (Adams \& Halchuk, 2003; Cui \& Atkinson, 2016). Both the tightly clustered and loosely clustered modes are described by steep FMDs and potentially higher $b$-values, indicating that they contain a larger proportion of smallermagnitude events. By contrast, the deep-background has an estimated $b$-value near unity, indicating more typical magnitude scaling. The differences between $b$-values remain within uncertainties; however, from the non-cumulative histograms it is quite clear that the deepbackground is characterized by a far more uniform distribution than the other components. The FMD for the entire catalogue (panel a) is largely governed by the loosely clustered subpopulation, given its dominant mixing proportion within the catalogue. 
a)

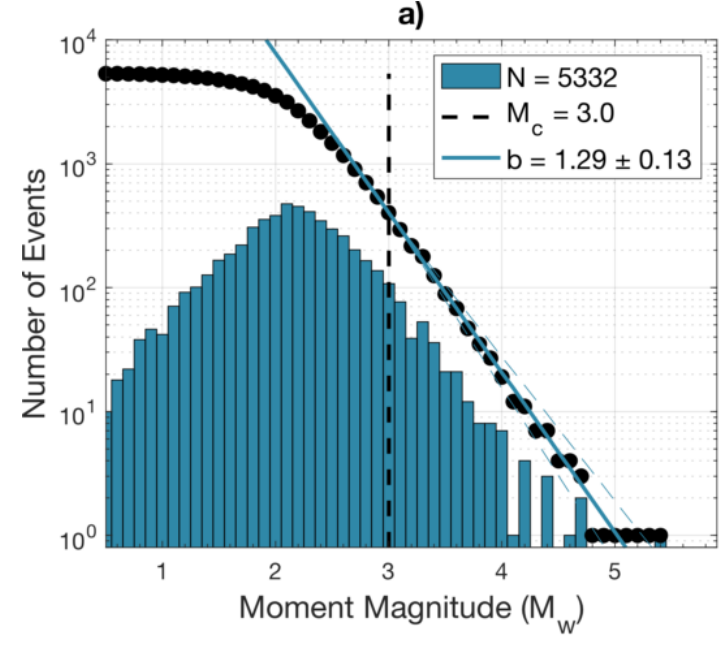

c)

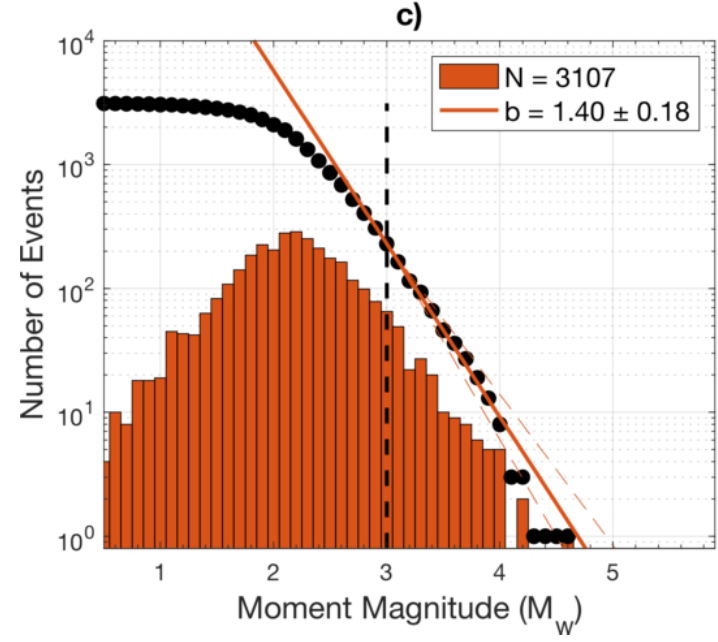

b)

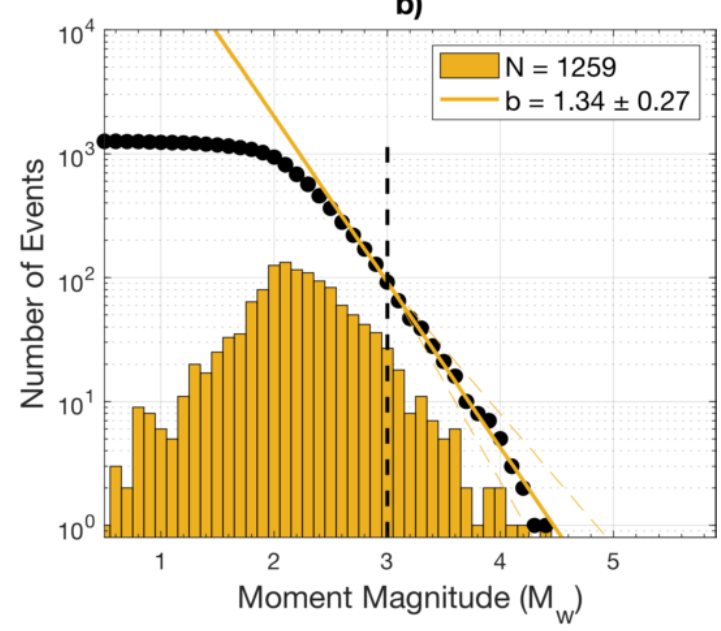

d)

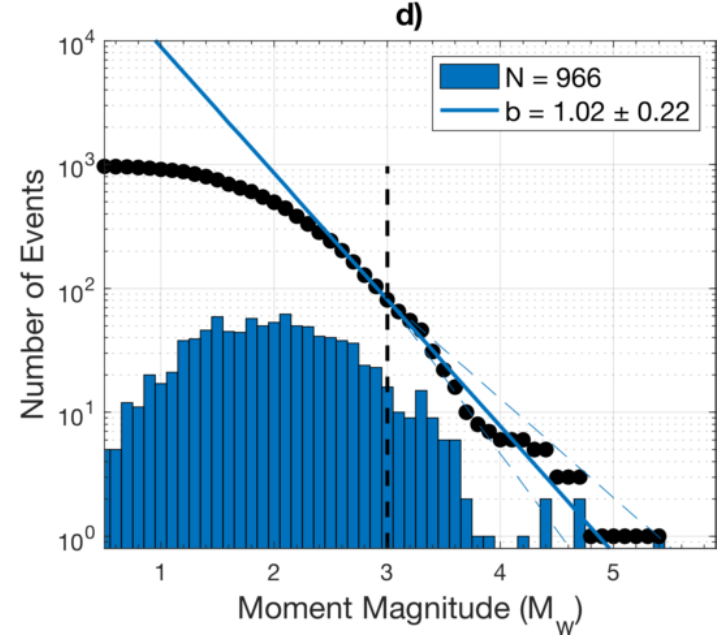

Figure 3-5: FMDs and estimated GR $b$-values of the regional WCSB catalogue (19752018) using $M_{c}=3.0$. Coloured histograms represent the non-cumulative distribution and black dots represent the cumulative distributions. Dashed black line indicates the cutoff magnitude. Coloured lines represent the modeled GR relations. a) Entire catalogue. b)

Tightly clustered mode. c) Loosely clustered mode. d) Deep-background.

A spatial map of the catalogued events is displayed in Figure 3-6. Blue markers represent deepbackground events, while orange and yellow markers represent the loosely clustered and tightly clustered events, respectively. Dashed boxes surround individual areas of suspected induced activity (several of which are investigated in Chapter 4). According to the NND algorithm, both the blue and orange markers are classified as the broad "background" portion of events. However, it is found here that the two subpopulations are not representative of the same type of 
seismicity. As a physical representation of the results in Figure 3-4, the blue markers are substantially more evenly distributed and seemingly reflective of the natural tectonic background (the majority occur along the foreland belt of the Rocky Mountain range), whereas an obvious spatial dependence on the distribution of orange and yellow markers is indicative of seismic clustering. Note that orange and yellow markers overwhelmingly dominate the dashed boxes. 


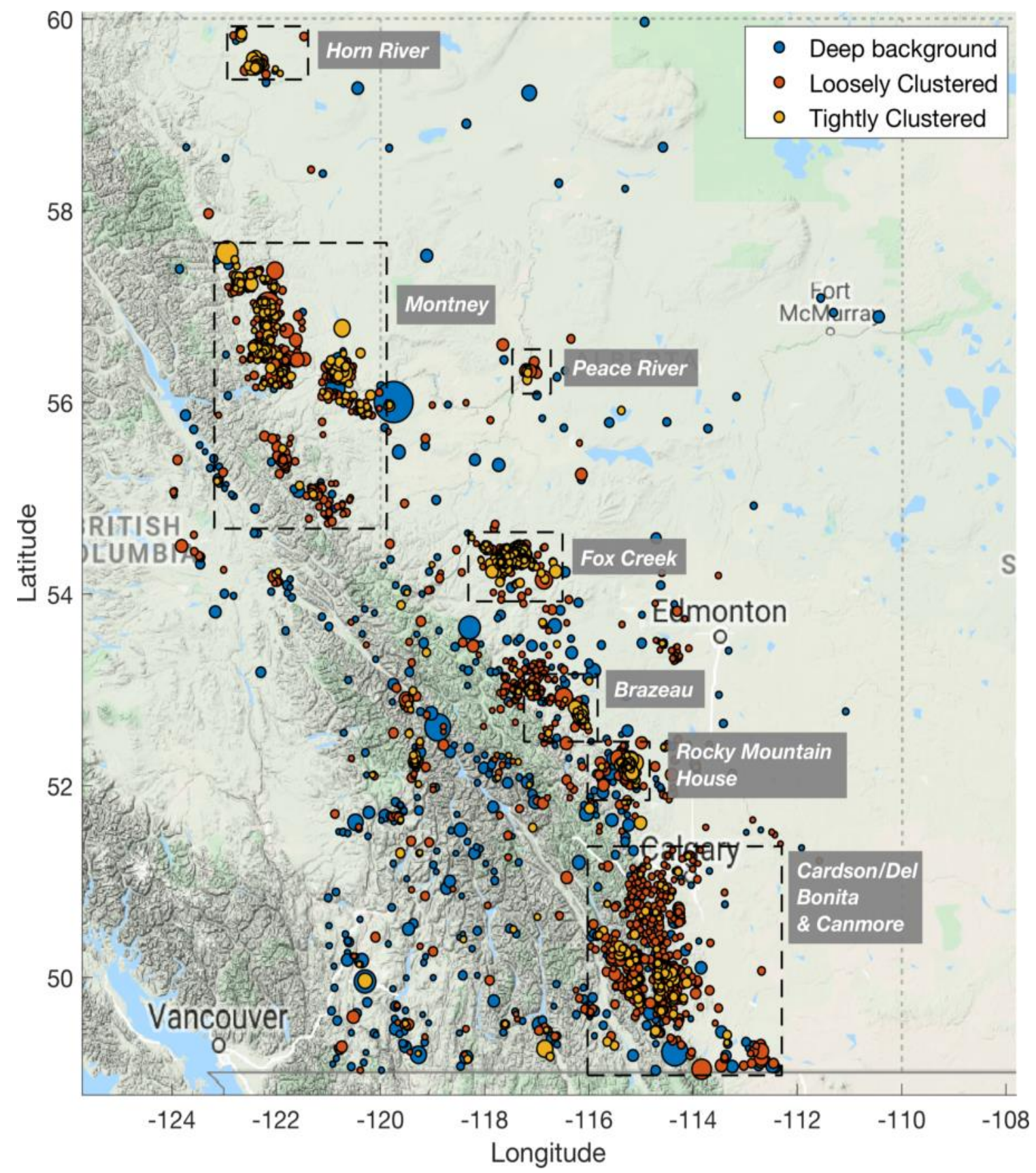

Figure 3-6: Spatial map of earthquakes for the regional WCSB catalogue (1975-2018) using $M_{0}=2.0$, represented in terms of their nearest-neighbour distance categorization. Blue markers are the deep-background, orange markers are the loosely clustered events and yellow markers are the tightly clustered events. Marker size indicates magnitude.

Dashed boxes surround areas of suspected induced clustering. 
Within the NND model's definition of event families, any earthquake has the potential to generate offspring, but only background earthquakes (here both loosely clustered and deepbackground events) can initiate a sequence as "root" or "ancestor" events. It is observed here that the background events occurring farther away from their own potential parent are less likely to initiate a clustering sequence, with deep-background events substantially less likely to trigger seismicity than loosely clustered events. Figure 3-7 plots the cumulative number of unique ancestors corresponding to non-single event families ( $N \geq 2$ events, panel a) and significant event families ( $N \geq 5$ events, panel b), across a range of $\eta$ values. 
a)

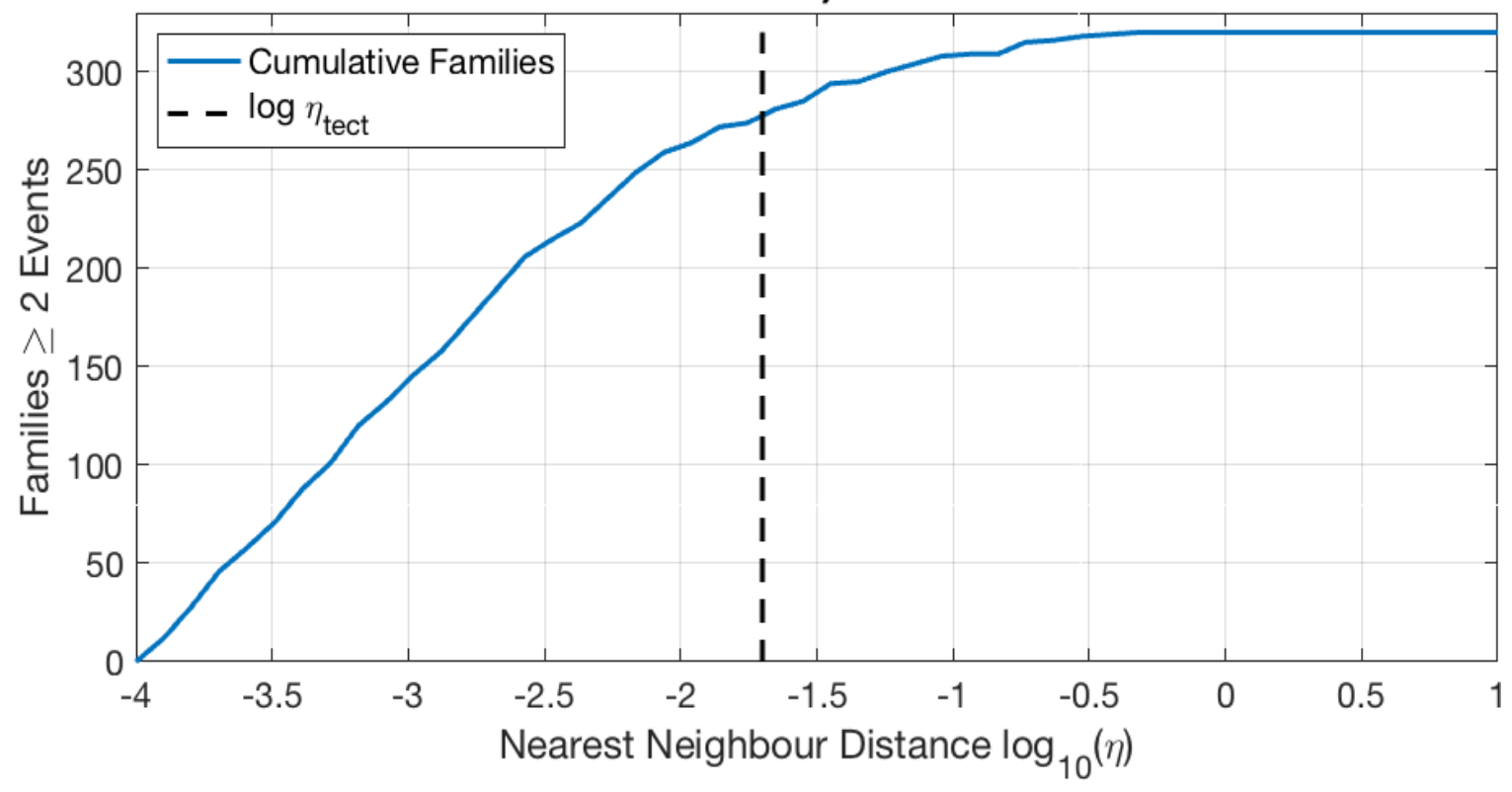

b)

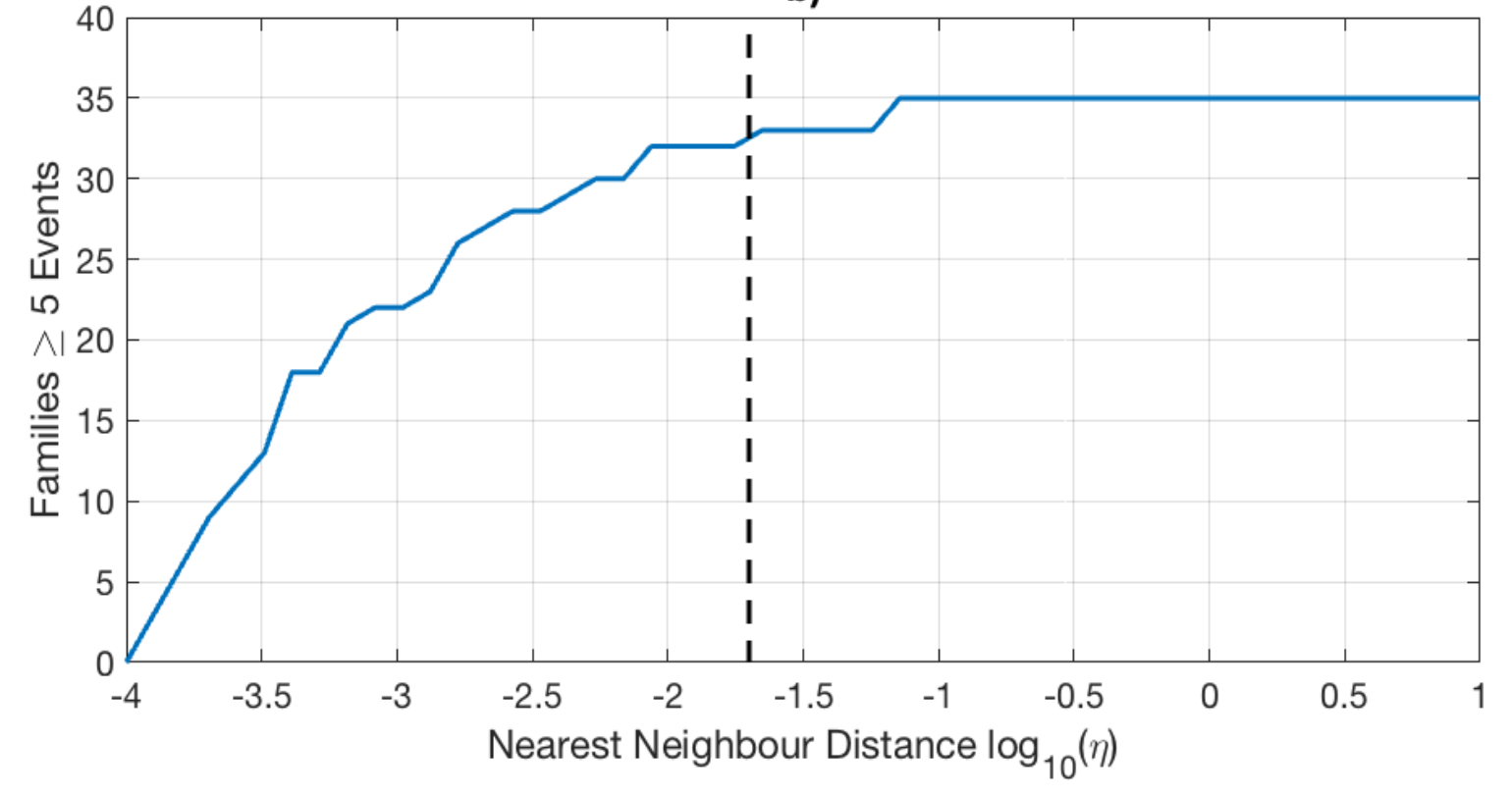

Figure 3-7: Cumulative number of detected event families vs. the $\boldsymbol{\eta}$ values of their ancestor event (blue line). Dashed black line represents $\log _{10} \eta_{\text {tect }} \approx-1.7$, the separation point between the two background modes. a) Non-single event families $(N \geq 2)$. b) Significant event families $(N \geq 5)$. 
Out of the 320 non-single event families, 281 (88\%) begin from the loosely clustered mode and $39(12 \%)$ begin from the deep background. Out of the 35 identified significant sequences, 33 $(94 \%)$ initiate from loosely clustered ancestors while only $2(6 \%)$ originate from the deepbackground. This can be seen in the distinctive tapering-off of the number of cumulative event families that occurs near the cut-off $\log _{10} \eta_{\text {tect }} \approx-1.7$ in both panels. On the other hand, it is true that there is a larger population of loosely clustered events, which could partially explain the disparity. However, even relative to the mixing proportion of each mode, a non-single event family is twice as likely, and a significant event family is nearly four times as likely to have originated from the loosely clustered mode rather than in the deep-background. This may be observed in Figure 3-6; overlap occurs substantially between loosely clustered (orange) and tightly clustered (yellow) markers, but rarely occurs between deep-background (blue) and tightly clustered (yellow) markers. Overall, the differences between the loosely clustered and deep-background subpopulations appear noteworthy and demonstrable.

\subsection{Separated Time Periods (1975-2009, 2010-2018)}

Figure 3-8 presents the joint $(T, R)$ distributions of the WCSB for separated time intervals. From 1975-2009, the regional catalogue is characterized by natural activity and isolated cases of induced clustering due to conventional oil and gas production and associated wastewater disposal, primarily within the Duvernay and Montney formations (Wetmiller, 1986; Horner et al., 1994; Baranova et al., 1999; Schultz et al., 2014). The resulting space-time inter-event distance distribution shows that both background modes are dominant, containing $83 \%$ of all events analyzed, indicating mainly single events and loose clustering, but little tight clustering (panel a). In contrast, the regional catalogue between 2010-2018 is characterized by sparse natural activity, likely due to the much shorter time-frame, and large amounts of induced activity attributed to the sharp rise in the implementation of horizontally drilled HF operations within the Duvernay, Montney, Cardium and Bakken formations, among others (B.C. Oil and Gas Commission, 2012, 2014; Schultz et al., 2015a, 2015c; Atkinson et al., 2016; Bao \& Eaton, 2016). Similar to the study of Oklahoma by Vasylkivska \& Huerta (2017), the joint distribution changes strikingly, as the deep-background subpopulation nearly disappears and a tightly clustered mode arises, indicating both substantial loosely clustered and tightly clustered seismicity (panel b). An important limitation worth mentioning is the variation in seismic 
monitoring capability over time; many more stations were operational during the recent time period, which undoubtedly had an effect on the distributions. The FMDs in Figure 3-5 illustrate that both the tightly clustered and loosely clustered subpopulations also appear to manifest more often at smaller magnitudes. However, it is shown below that the changes in inter-event distance distributions across time are vastly disproportionate between modes, and cannot be entirely due to improvement in network detection. Moreover, in Appendix B, a sensitivity analysis demonstrates that the differences hold for cut-off magnitudes up to the regional completeness level. 

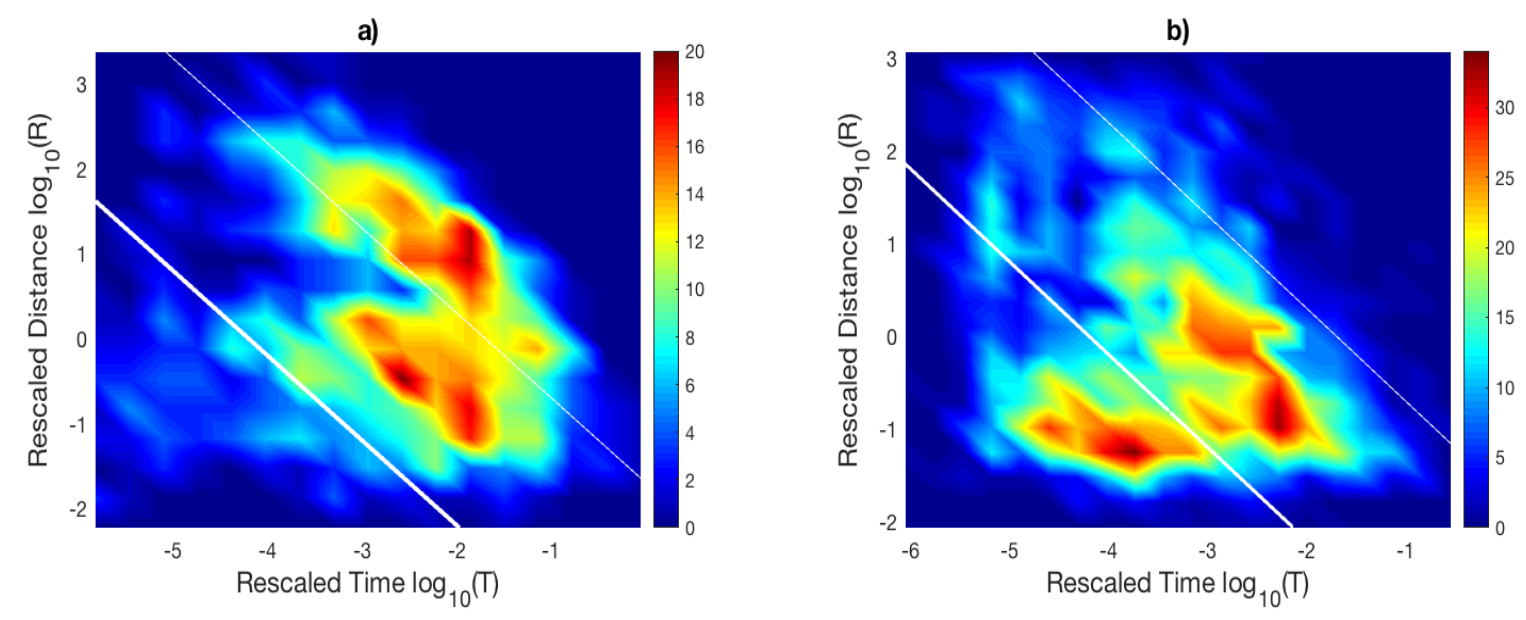

c)
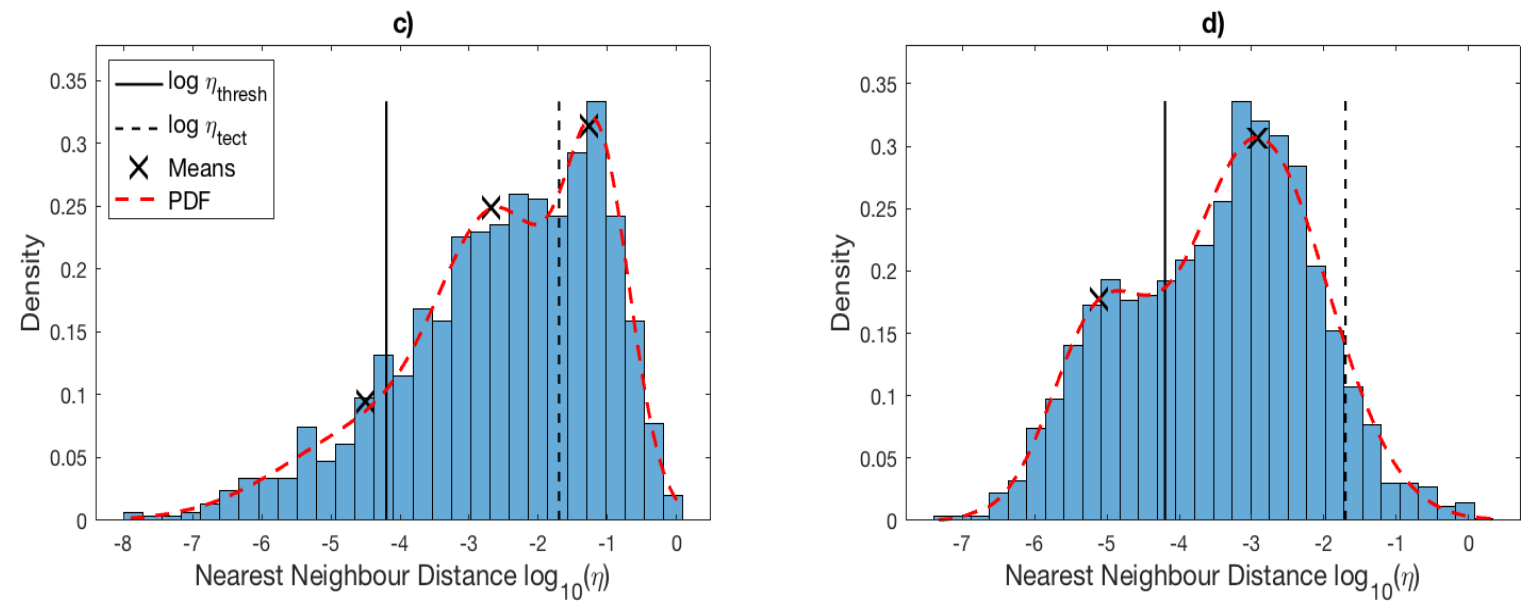

Figure 3-8: Comparison of NND distributions of the regional WCSB catalogue across time using $M_{0}=2.0$. (a, b) Joint distributions of the temporal and spatial components $(T, R)$. Bold white line indicates the threshold $\log _{10} \eta_{\text {thresh }}$ between tightly clustered and loosely clustered components. Thin white line indicates the threshold $\log _{10} \eta_{\text {tect }}$ between loosely clustered and deep-background components. Colour bar indicates frequency of occurrence. (c, d) Normalized densities of $\eta$ values. Solid black line is $\log _{10} \eta_{\text {thresh }}$ and dashed black line is $\log _{10} \eta_{\text {tect }}$. Dashed red line is the normalized probability density function of the Gaussian mixture and black crosses are the component means. (a, c) 19752009, both background modes are dominant. (b, d) 2010-2018, deep-background shrinks while tightly clustered mode appears. The loosely clustered subpopulation is common to both time frames. 
Results of the GMM analyses for both periods are summarized in Table 3-3. The analysis over the first period once again identifies all three modes; over the second period it picks out only the dominant 2 , the tightly clustered and loosely clustered components. This is understandable, as the deep-background shrinks and ultimately contains only $7.5 \%$ of the mixing proportion from 2010-2018 (Table 3-4). Note that event counts within subpopulations for separated time frames do not sum exactly to those found over the entire period. This is a consequence of time windowing, as applying a temporal cut-off can both sever and create new branches between potential family members. Zaliapin and Ben-Zion (2013a, supplementary document) show that between 80 and $90 \%$ of events retain membership to the same event family, though their direct parent may be reassigned.

Table 3-3: EM results for the GMM applied to the separated time periods using $M_{0}=$ 2. 0. $k$ is the number of modes, AIC and BIC are the resulting Akaike and Bayes Information Criteria. Highlights show the minimized values.

\begin{tabular}{ccccc}
\hline & \multicolumn{2}{c}{ 1975-2009 } & \multicolumn{2}{c}{ 2010-2018 } \\
\hline $\boldsymbol{k}$ & AIC & BIC & AIC & BIC \\
\hline $\mathbf{1}$ & 3852.15 & 3862.09 & 8355.68 & 8367.30 \\
\hline $\mathbf{2}$ & 3709.98 & 3734.82 & 8219.14 & 8254.19 \\
$\mathbf{3}$ & 3670.41 & 3710.16 & 8223.26 & 8265.49 \\
\hline $\mathbf{4}$ & 3673.16 & 3727.82 & 8224.55 & 8288.10 \\
\hline
\end{tabular}


Table 3-4: NND Breakdown for the separated time periods using $M_{0}=2.0$.

Tightly Clustered Loosely Clustered Deep-Background

$\begin{array}{llll}1975-2009 & 177(16.6 \%) & 518(48.7 \%) & 369(34.7 \%)\end{array}$

$2010-2018 \quad 842(34.1 \%) \quad 1440(58.4 \%) \quad 185(7.5 \%)$

Overall, there is a $27 \%$ drop in the relative number of deep-background events and a $10 \%$ increase in the proportion of loosely clustered events between the two time periods. There is a $17.5 \%$ increase in the population of tightly clustered events; the absolute increases in the number of tightly and loosely clustered events are substantial (177 to 842 and 518 to 1440). The vastly disproportionate changes in rate between sub-populations indicate that the sharp rise in tightly and loosely clustered events cannot be fully explained by improvement in network detection capability alone. While the yearly detected $M \geq 2$ deep-background rate roughly doubles, the equivalent loosely and tightly clustered rates increase tenfold and seventeen-fold, respectively. A two-sample Kolmogrov-Smirnov test was performed on the distributions of $\eta$ values determined for the two temporal subsets, in order to test the null hypothesis that the differences observed are within sampling errors and they are actually from the same continuous distribution. The test rejects the hypothesis at the $1 \%$ significance level.

These results suggest that the changes in the inter-event distance distribution across Alberta and eastern B.C. over time are statistically significant and likely not naturally occurring. The decreased mixing proportion of deep-background $\left(\log _{10} \eta \geq \log _{10} \eta_{\text {tect }}\right)$ events indicate that the majority of the recent seismicity is not tectonic, while the increase in tightly clustered $\left(\log _{10} \eta<\log _{10} \eta_{\text {thresh }}\right)$ seismic activity correlates temporally with the rise in horizontally drilled HF treatments within the region. Figure 3-6 shows that clustering is highly spatially correlated with human activity as well, with smaller inter-event distances $\left(\log _{10} \eta<\right.$ $\log _{10} \eta_{\text {tect }}$, i.e. orange and yellow markers) transpiring chiefly near areas flagged as suspicious. The statistical properties of several of these suspicious areas are explored in detail in the next chapter. 


\section{Chapter 4}

\section{Case-by-case Investigation of Seismic Clustering within the Western Canada Sedimentary Basin}

In the previous chapter, three important aspects of the regional catalogue were demonstrated. Firstly, a statistical difference exists between the background seismicity potentially generated through natural tectonic processes (interpreted as the "deep-background") versus that primarily stimulated as a by-product of unnatural, human-induced forces (the "loosely clustered" earthquakes). Namely, the loosely clustered events are distributed more closely together in both space and time and are more likely to initiate tightly clustered event sequences (Figure 3-7) than the deep-background, which resembles a time-stationary, space-inhomogeneous marked Poisson (SIP) process (Figure 3-4). It is possible that this distinction between background modes occurs in other regions as well (Figure 5-2 \& Figure 5-3; discussed in Chapter 5). Secondly, the loosely clustered population appears as a common feature among cases of induced seismicity in the WCSB independent of the stimulating mechanism, and can be observed both in event maps (Figure 3-6) and in Nearest-Neighbour Distance (NND) distributions over time (Figure 3-8). Thirdly, in the past decade a disproportionate increase in the loosely and tightly clustered earthquake subpopulations is observed in comparison with the deep-background occurrence rate, which correlates spatially and temporally with the implementation of unconventional extraction technology (HF) (Figure 3-8 \& Table 3-4).

This chapter presents the analyses of the statistical properties of four distinct cases of induced seismic clustering within the Western Canada Sedimentary Basin (WCSB). Each cluster was examined individually; first the local completeness magnitude $\left(M_{c}\right)$ was estimated using the techniques summarized in Chapter 2.3 and the Gutenberg-Richter (GR) parameters were computed with their associated uncertainties. The NND model was then applied using the determined $b$-value, and the resulting mixing proportions of the loosely and tightly clustered modes were evaluated. The tightly clustered events were linked to form event families and to identify possible foreshocks, mainshocks, aftershocks, and aftershocks of aftershocks (higher order triggers), as well as to isolate single events. The relative proportions of these event types were reviewed, and from them, temporal, spatial and topological family-tree structures were 
constructed for each significant sequence. The types of sequences (burst or swarm) present were then determined using the parameters discussed in Chapter 2.5.3. Finally, the Epidemic Type Aftershock Sequence (ETAS) model was fitted to each dataset, and the resulting parameters and quality-of-fits (QOFs) were evaluated.

For each case study, the low magnitude cut-off $M_{0}=2.0$ and constant value $d_{f}=1.5$ for the NND analyses were maintained. The regionally derived threshold value $\log _{10} \eta_{\text {thresh }}=-4.2$ was used as the separation point between loosely and tightly clustered modes. The GR and ETAS models are sensitive to the completeness level, and hence were applied using events greater than or equal to the estimated local $M_{c}$.

\subsection{Rocky Mountain House Cluster (RMHC) - Conventional Gas Extraction (1975-2000)}

The RMHC was one of the first significant instances of induced seismicity within the WCSB and occurred near the oldest production zone in the region operating primarily within the Duvernay formation, approximately $25 \mathrm{~km}$ southwest of the town of Rocky Mountain House. The area had been historically quiescent before the onset of production in the early 1970s (Rogers \& Horner, 1991). It became active predominantly from 1975-1992, where it lagged peak production rates by approximately 5 years. Several wastewater disposal wells were operational in the area during this time as well. However, Wetmiller (1986) found that the majority of events occurred roughly within a $15 \mathrm{~km}$ radius and were concentrated close to the Strachan and Ricinus gas fields, while very little seismicity occurred around the nearby Ferrier and Caroline oil fields and injection wells. Baranova et al. (1999) proposed that the earthquakes were triggered due to long-term compaction-related changes in the stress field caused by the extraction of fluid. Following the active period, there was a considerable reduction in clustered seismicity coinciding with the decline in production, with an apparent return to minor background activity by the year 2000. This cluster was analyzed over the chiefly active period between 1975-2000 using a radial distance of $20 \mathrm{~km}$ centered at [-115.24, 52.17] (Figure 4-1). 


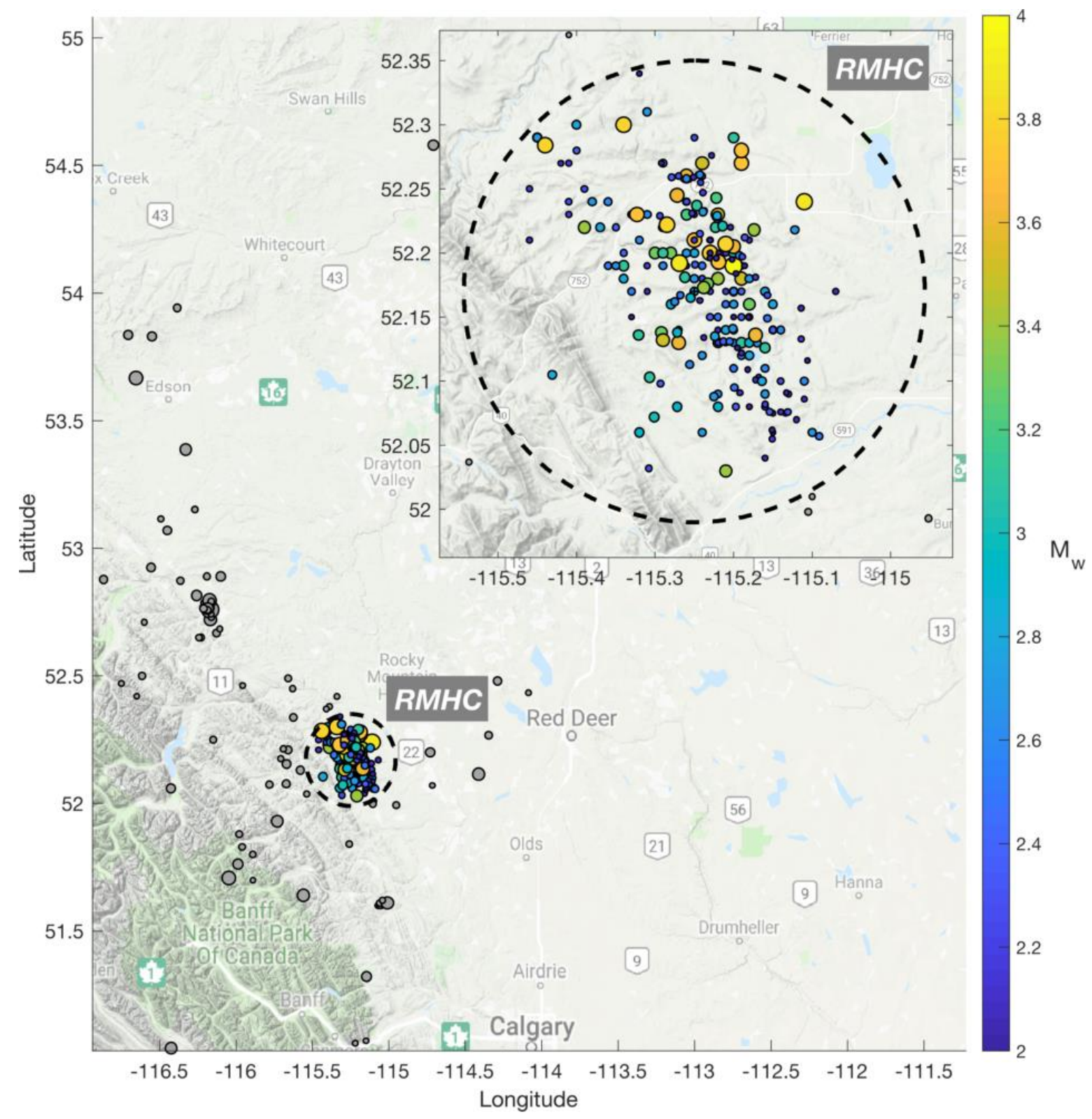

Figure 4-1: Map of the Rocky Mountain House cluster study area between 1975-2000.

Dashed circle represents a $20 \mathrm{~km}$ radius from the coordinates [-115.24, 52.17]. Markers are seismic events. Coloured markers are the data points used for analysis.

The results of the completeness magnitude $\left(M_{c}\right)$ and Gutenberg-Richter (GR) analyses are provided in Figure 4-2 and summarized in Table 4-1. The shaping of the non-cumulative histogram is broad, resulting in a shallow sloping of the cumulative FMD. It is worth noting that this particular study area has undergone several network changes since the late 1970s (see Cui \& Atkinson, 2016), due in part to the fluctuating seismic activity, which may explain the 
observed kinks in the distribution. The point of maximum curvature (MAXC) occurs early in the histogram (Figure 4-2, panel a) and is most likely an underestimation. The $b$-value Stability Method's (MBS) rolling average $\left(b_{a v e}\right)$ ascends gradually between cut-off magnitudes $1.9 \leq$ $M_{0} \leq 2.6$, after which it increases rapidly as the cumulative FMD drops off (orange line with triangle markers, panel c). There are two periods of relative $b$-value stability, the first between $1.9 \leq M_{0} \leq 2.3$ and the second between $2.6 \leq M_{0} \leq 2.9$ (blue line, panel c). The second period appears to be accounting for the dataset drop-off above M3.7 with a steeper slope (higher $b$-value) while the first period primarily models the observed seismicity. The Goodnessof-Fit Test (GFT) computes a maximum correlation between the synthetic and observed FMD at $M=2.3$ (panel b).

The GFT $M_{c}$ matches the FMD visually and lies well within the bounds of the MBS method. It also corresponds with the first period of $b$-value stability and the only period of $b_{\text {ave }}$ stability. The GR parameters and their uncertainties were therefore computed using $M_{c}=2.3$ (panel d and Table 4-1). As expected from the broad magnitude distribution, the $b$-value is low, suggesting a preponderance of larger magnitude events and fewer than typical smaller magnitude events. From the FMD, it can be seen that this is true only up to a certain point, as there is a significant drop-off in the data sample for magnitudes above M3.7. 
a)

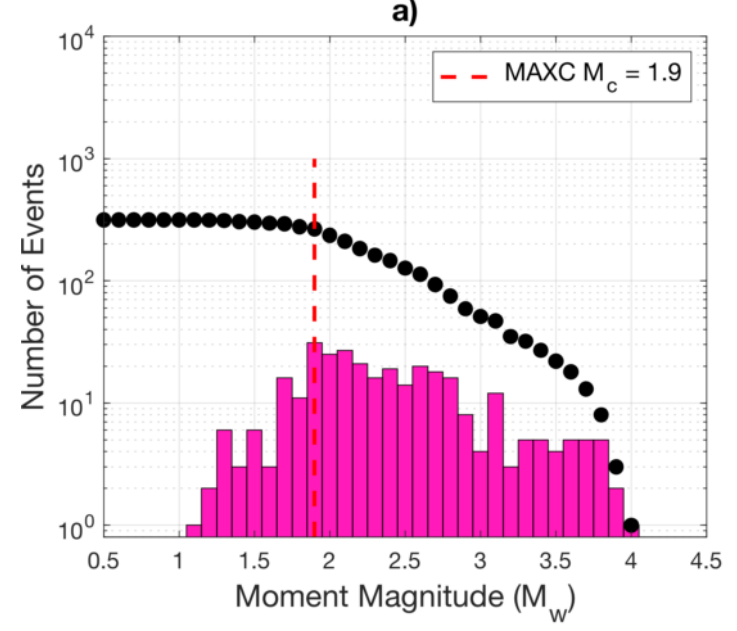

c)

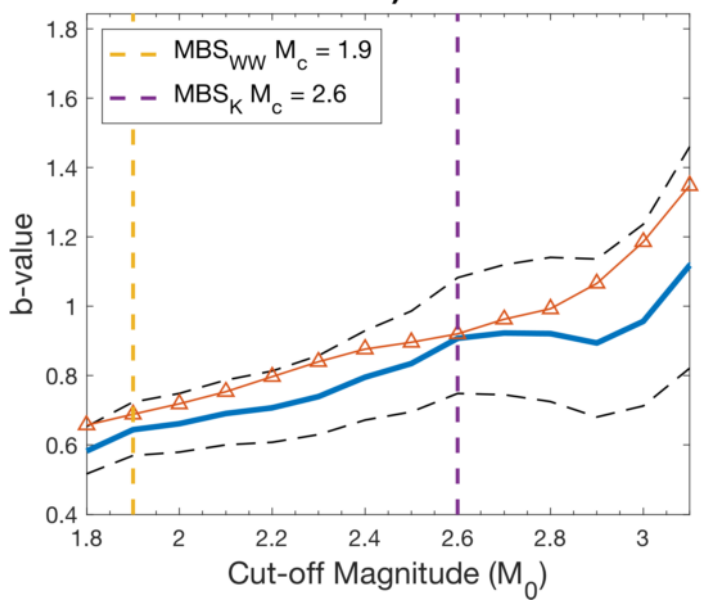

b)

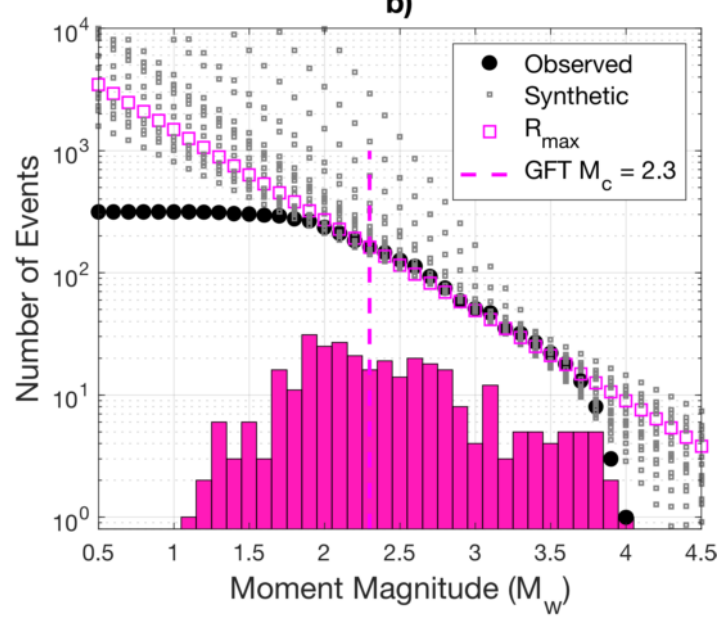

d)

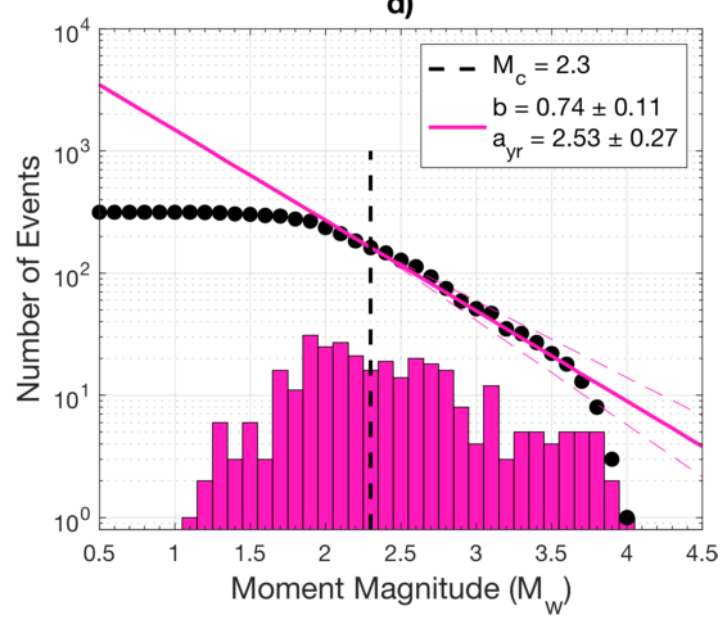

Figure 4-2: $M_{c}$ and GR Analyses for the Rocky Mountain House cluster. a) Maximum Curvature Method (MAXC). b) Goodness-of-Fit Test (GFT). c) Method of $b$-value Stability (MBS). d) GR parameter estimation using determined Mc.

Table 4-1: Summary of $M_{c}$ and GR Analyses for the Rocky Mountain House cluster.

$\begin{array}{cccccccc}\text { N } & \text { MAXC } & \text { GFT } & \text { MBSww } & \text { MBSk } & \text { Determined } M_{\boldsymbol{c}} & \text { GR } \boldsymbol{b} & \text { GR } \boldsymbol{a} \\ \mathbf{3 1 4} & 1.9 & 2.3 & 1.9 & 2.6 & 2.3 & 0.74 \pm 0.11 & 2.53 \pm 0.27\end{array}$

Figure 4-3 presents the results of the NND model application to the RMHC using the $b$-value determined above. The distribution contains a high mixing proportion of middle-mode events, indicative of an elevated background rate and the predominance of loosely clustered seismicity. 
There is a notable absence of a tightly clustered mode in the joint distribution (panel a), but a small subpopulation can be observed in the $\eta$ density distribution (panel b) indicating a low number of tightly grouped event sequences.

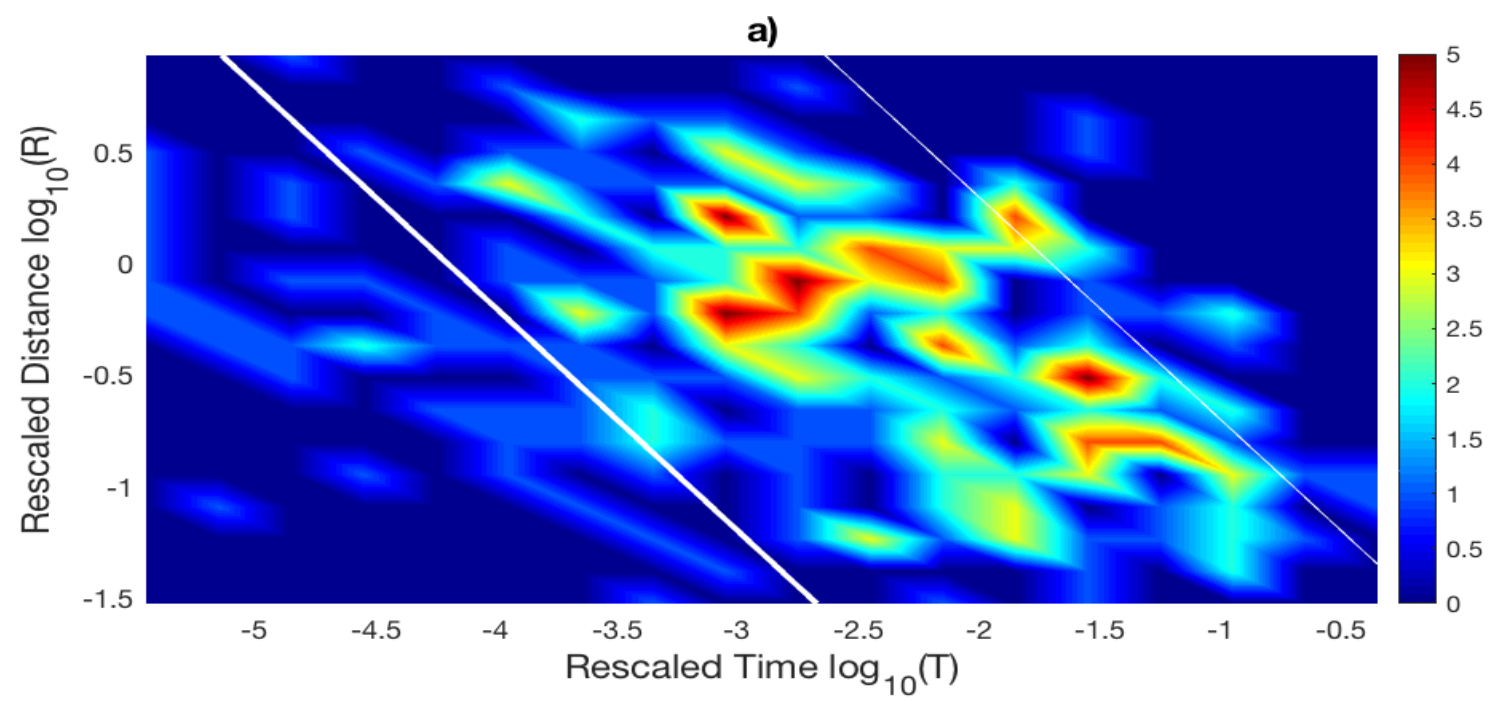

b)

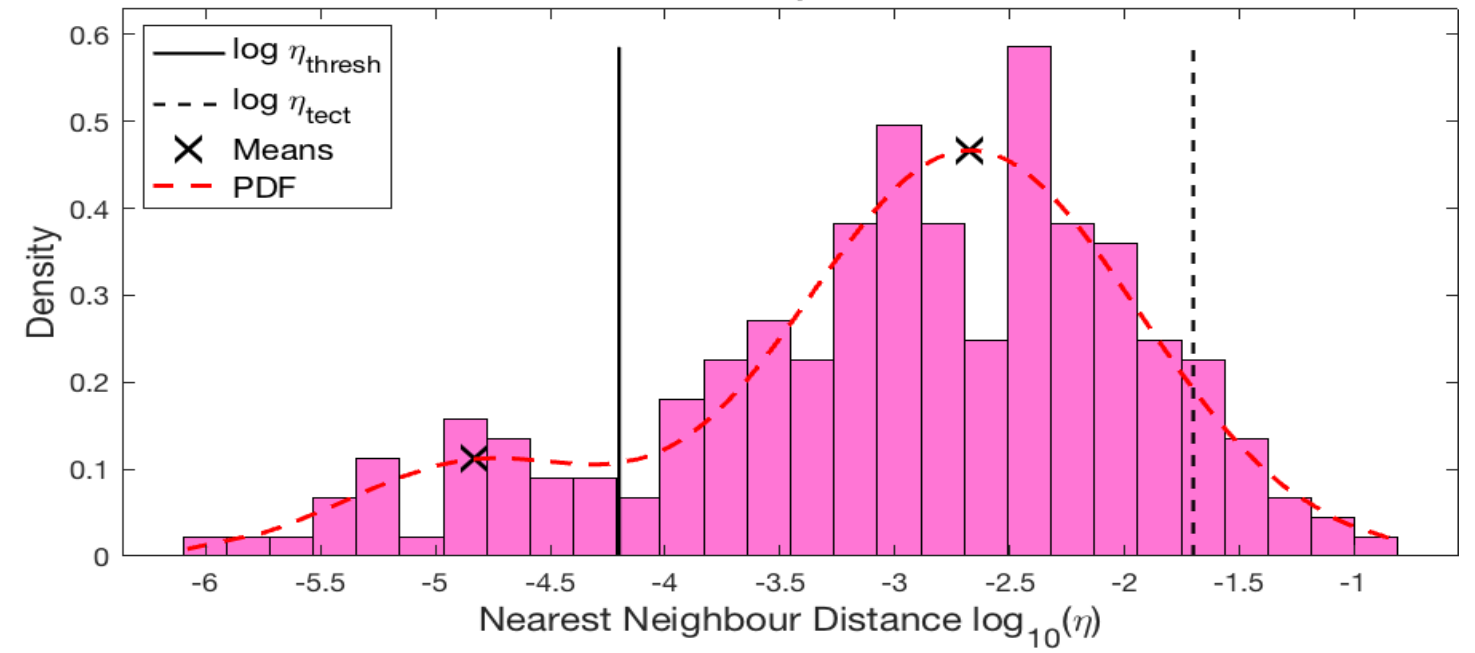

Figure 4-3: Nearest-Neighbour Distance distribution for the Rocky Mountain House cluster. a) Joint distribution of the temporal and spatial components $(T, R)$. b) Normalized density of $\boldsymbol{\eta}$ values.

Table 4-2 summarizes the results of the event-family discretization process, where strongly linked events are connected and classified as foreshocks, mainshocks and aftershocks, and weakly linked events are separated as singles. A fifth category is included in this study, 
"aftershocks-of-aftershocks", which counts the number of second-order or higher offspring events linked to a mainshock. As suggested by Figure 4-3, there are a high proportion of singles due to the dominance of the loosely clustered background and much smaller clustered mode. Note again that while all singles are background events, not all background events are singles (they may be the initiators of event sequences).

Table 4-2: Event-Family Breakdown for the Rocky Mountain House cluster.

\section{Single Events Foreshocks Mainshocks Aftershocks Aftershocks of Aftershocks}

$185(78.7 \%) \quad 4(1.7 \%) \quad 19(8.1 \%) \quad 27(11.5 \%) \quad 2(0.9 \%)$

Figure 4-4 displays the magnitude-time evolution of event families across the active period. White squares represent detected singles, red circles are foreshocks, red triangles are mainshocks, blue circles are aftershocks, and green circles are higher order aftershocks. Grey lines link event-family members together. The vast majority of events are unlinked singles (185; 79\%), which do not appear to directly trigger further detectable seismicity. Out of the 204 total background events (possible ancestors), only 19 trigger at least one other detected event, despite the existence of over $50 \mathbf{M} 3+$ events in the cluster. There are paucities of foreshocks (4; $1.7 \%)$ and aftershocks-of-aftershocks $(2 ; 1 \%)$. These factors indicate that the identified sequences tend to occur suddenly, without detectable precursory behavior, and then rapidly decay after the first generation of aftershocks without producing further significant clustering. 


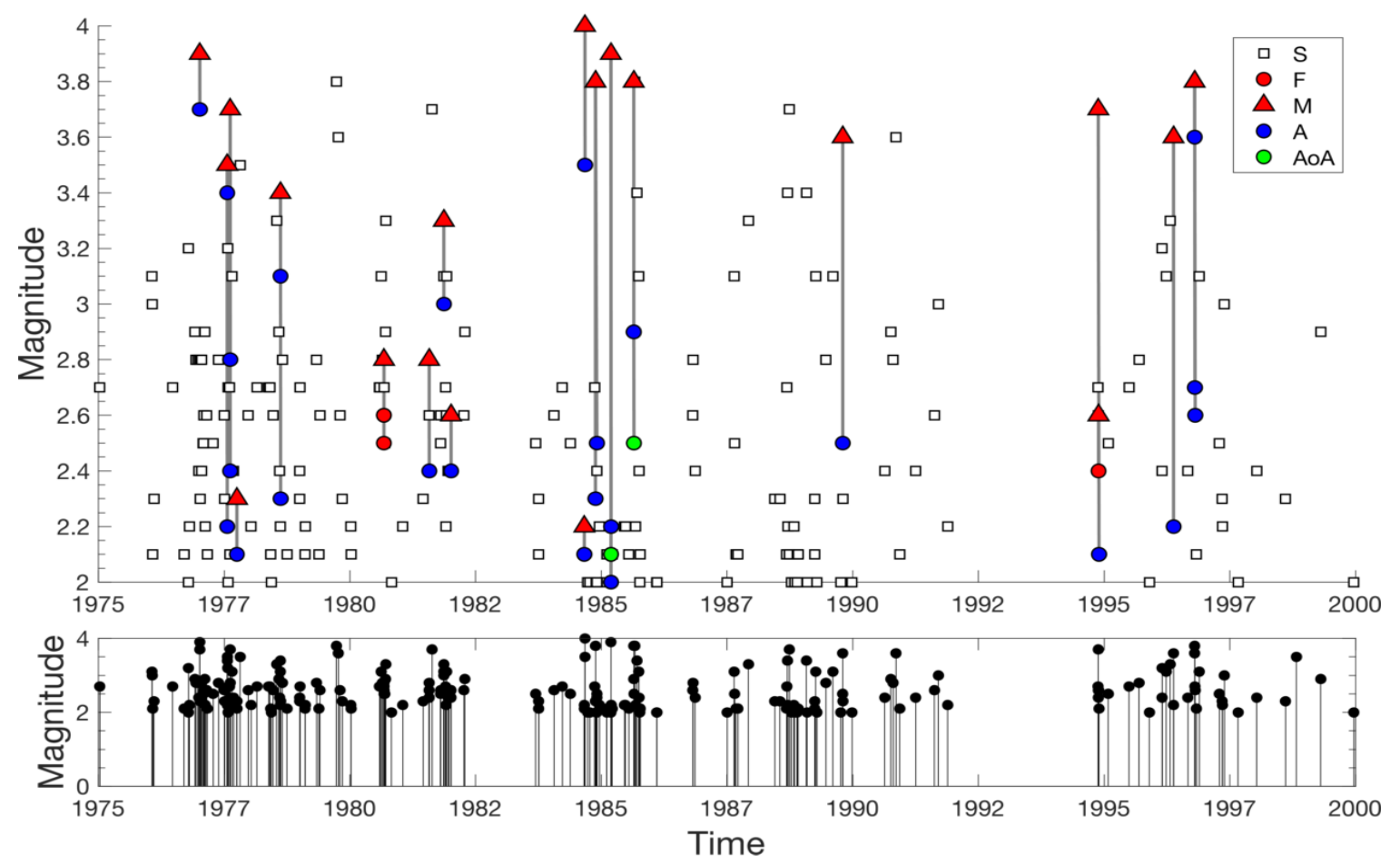

Figure 4-4: Magnitude vs. time evolution of event families within the Rocky Mountain House cluster. Lower plot: Event magnitudes vs. time. Upper plot: Event-type classification over time. White squares are singles, red circles are foreshocks, red triangles are mainshocks, blue circles are aftershocks, and green circles are aftershocks-ofaftershocks. Grey lines represent the strong links connecting individual event families.

Figure 4-5 arranges the detected significant sequences into their event family configurations, as discussed in Chapter 2.5.3. From left to right, they are displayed in magnitude-time evolution, spatial distribution and as topological structures. The event families are small; the largest contains only 5 events. 5 of the 6 families visually display the prototypical burst shape of minor aftershock sequences, with no foreshocks, a sudden M3.4+ mainshock causing some $1^{\text {st }}$ order aftershocks, and few higher order offspring. Sequence 2 is an outlier and is unclear, displaying neither typical swarm nor burst behavior. The parameter sets $\theta=\left\{\langle d\rangle, \delta, B, A, t_{D}, \Delta m\right\}$ for each sequence are presented in Table 4-3. The parameter values underscore the picture of lowmagnitude aftershock sequences, with low leaf depths and inverted branching numbers, spatial areas that scale roughly with the mainshock magnitude and short decay periods on the order of days. 
a)
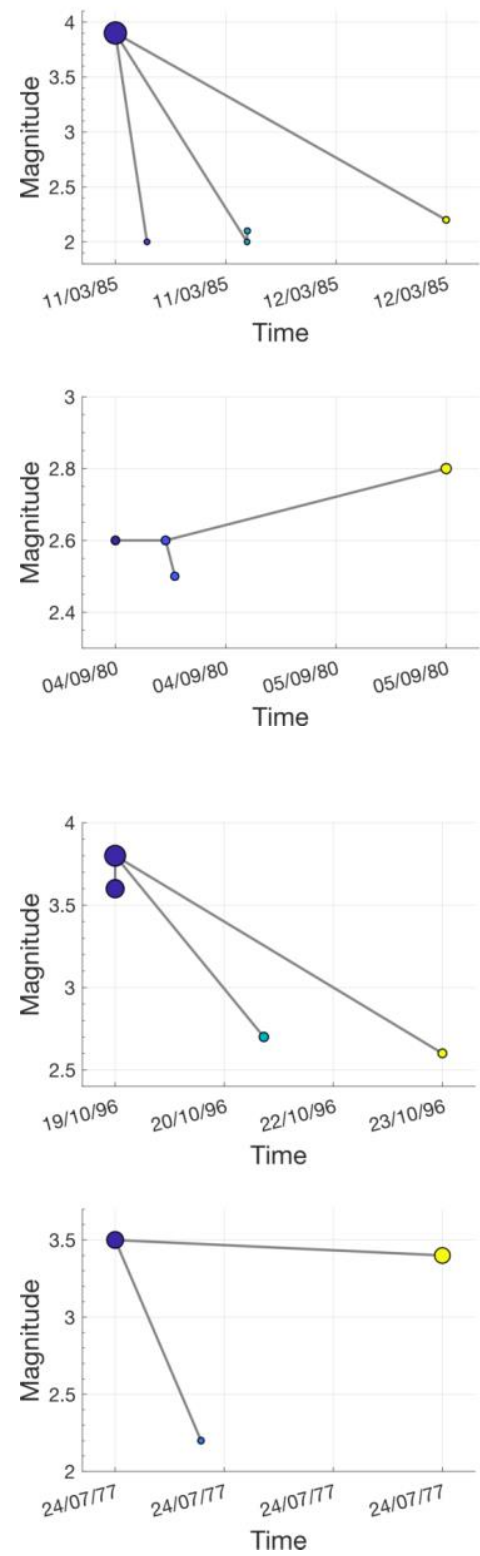

b)

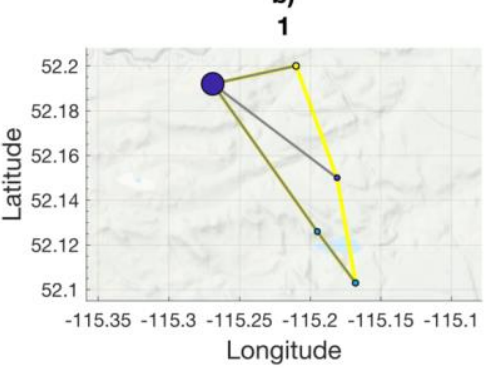

2

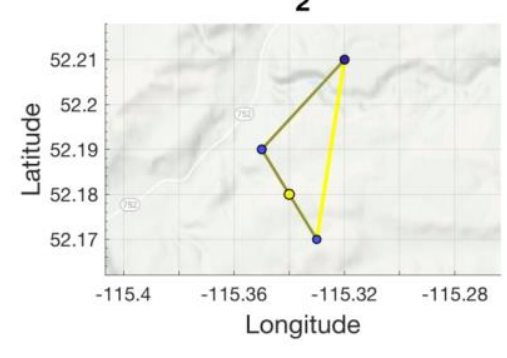

3
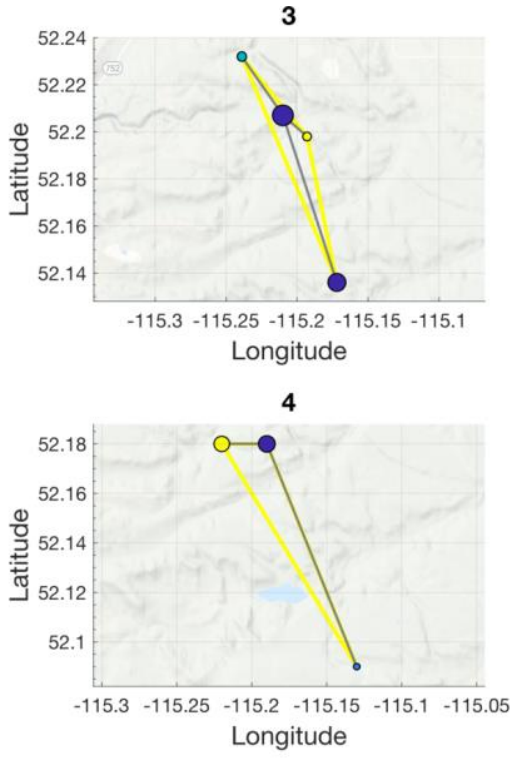

c)
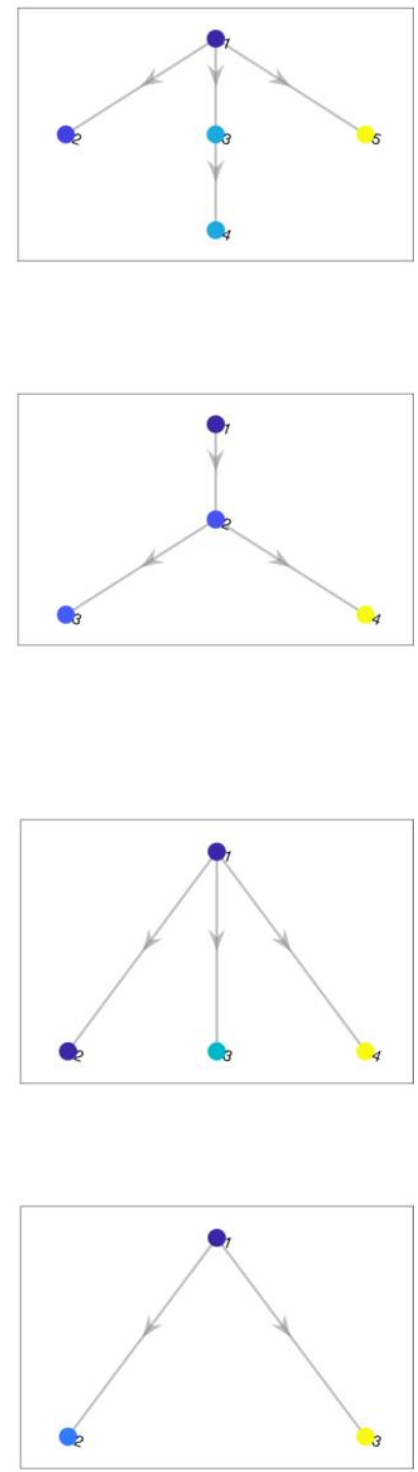

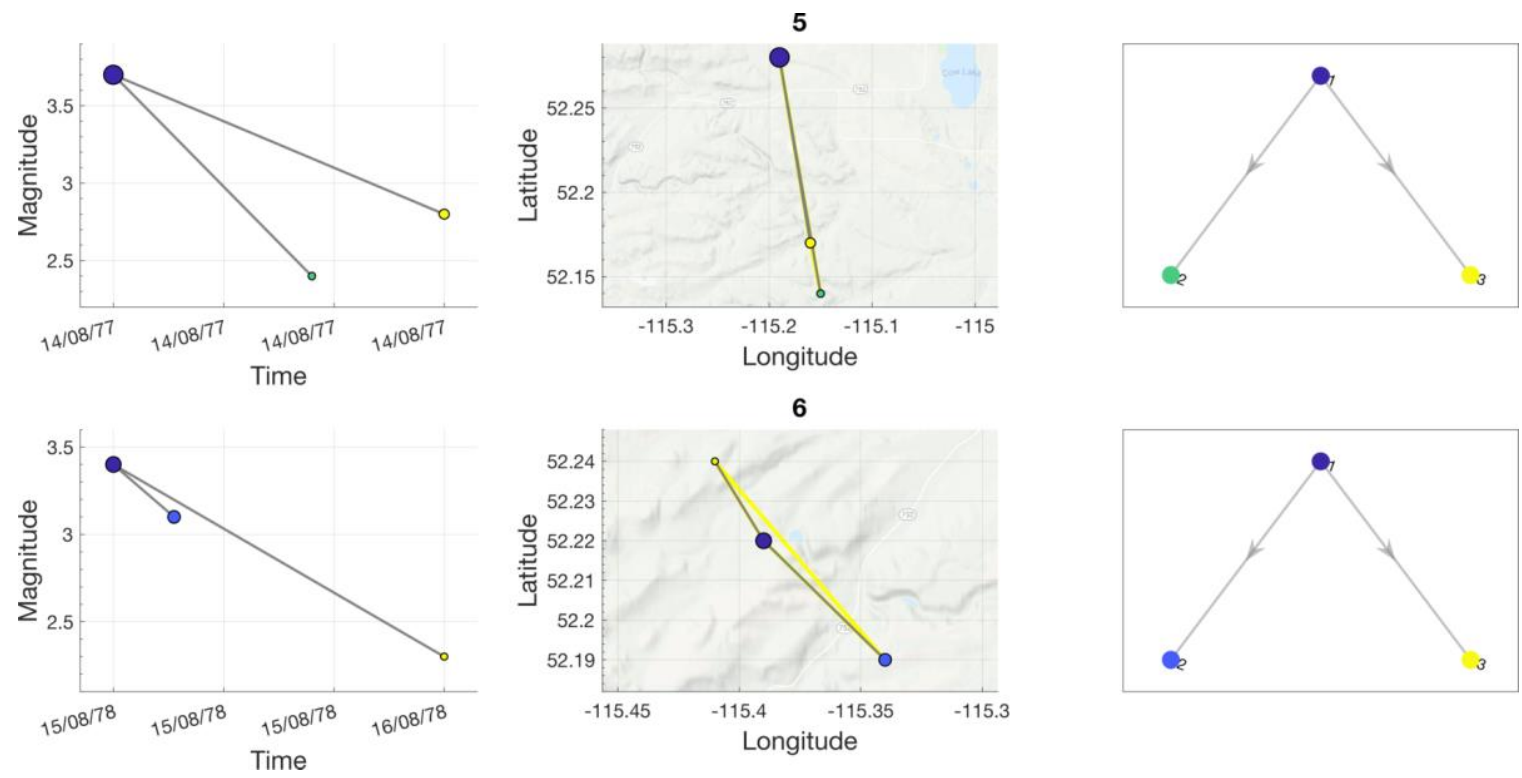

Figure 4-5: Event Family Structures in the Rocky Mountain House cluster. a) Moment magnitude vs. Time in days. b) Spatial map. Yellow border outlines the hull area occupied by the sequence. c) Directed tree graph in dimensionless space. Data points are coloured chronologically from darkest to lightest.

Table 4-3: Parameter sets for event families within the Rocky Mountain House cluster. $\mathbf{N}$ is the size of the sequence, $\langle d\rangle$ is the average leaf depth, $\delta$ is the normalized leaf depth, $B_{i}$ is the inverted branching number, $A$ is the spatial area, $t_{d}$ is the decay period, and $\Delta M$ is the magnitude differential.

\begin{tabular}{cccccccccc}
\hline Sequence & $\mathbf{N}$ & $\langle\boldsymbol{d}\rangle$ & $\boldsymbol{\delta}$ & $\boldsymbol{B}_{\boldsymbol{i}}$ & $\boldsymbol{A}\left(\mathbf{k m}^{2}\right)$ & $\boldsymbol{t}_{\boldsymbol{d}}$ (days) & $\boldsymbol{\Delta} \boldsymbol{M}$ & Class \\
\hline $\mathbf{1}$ & 5 & 1.33 & 0.60 & 0.50 & 25.98 & 1.26 & 1.70 & Burst \\
$\mathbf{2}$ & 4 & 2.00 & 1.00 & 0.67 & 3.79 & 0.75 & 0.20 & Unknown \\
$\mathbf{3}$ & 4 & 1.00 & 0.50 & 0.33 & 8.08 & 4.31 & 0.20 & Burst \\
$\mathbf{4}$ & 3 & 1.00 & 0.58 & 0.50 & 10.23 & 0.36 & 0.10 & Burst \\
$\mathbf{5}$ & 3 & 1.00 & 0.58 & 0.50 & 0.78 & 0.00 & 0.90 & Burst \\
\hline & 3 & 1.00 & 0.58 & 0.50 & 1.52 & 1.31 & 0.30 & Burst \\
\hline
\end{tabular}


In the final portion of analysis, the ETAS model was fitted to the RMHC using events greater than or equal to the determined completeness $\left(M_{c}=2.3\right)$. Figure 4-6 plots the observed rate and ETAS model in original time (panel a) and transformed time (panel b); visually the model is a poor fit. It under-predicts the seismicity throughout, which can be seen in both plots as the observed cumulative rate positively deviating from the model; it vaguely manages to capture the broader overall shape. A QOF of 0.072 was calculated, which is considered below average in this study; the resulting ETAS parameters are given in Table 4-4. 
a)

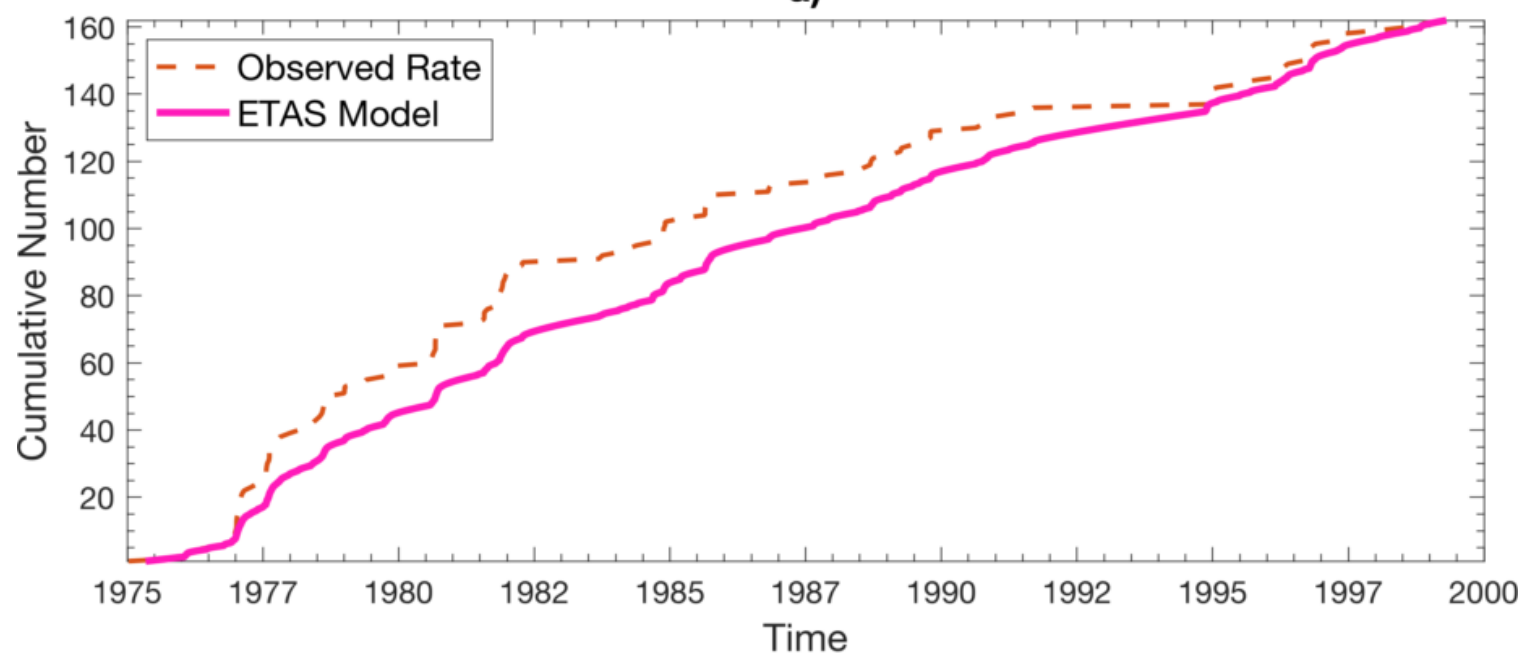

b)
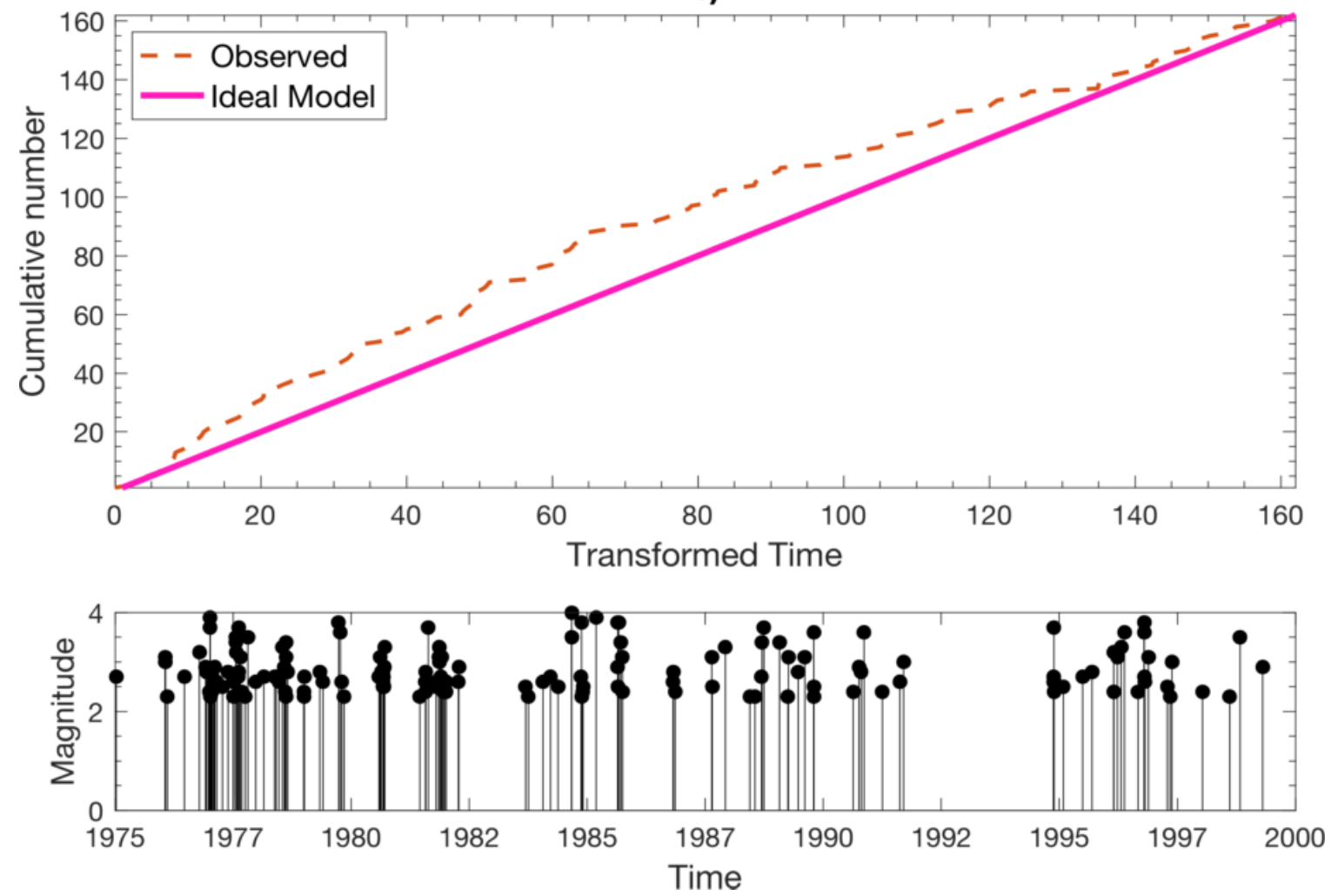

Figure 4-6: ETAS Model of the Rocky Mountain House cluster using $M_{c}=2.3$. a)

Original time plot. Dashed orange line is the observed cumulative rate of seismicity. Solid line is the optimized model. b) Transformed time plot. Solid line represents the "perfect" model fit. 
Table 4-4: ETAS model parameters for the Rocky Mountain House cluster. $\mathbf{N}$ is the number of events modeled, $\mu$ is the constant background rate, $K$ is the aftershock productivity, $c$ is the temporal offset, $p$ is the exponential decay, $\alpha$ is the magnitudeaftershock dependence, and QOF is the quality of the model fit.

\begin{tabular}{cccccccc}
$M_{\boldsymbol{c}}$ & $\mathbf{N}$ & $\boldsymbol{\mu}$ (Events/Day) & $\boldsymbol{K}$ (Events/Day) & $\boldsymbol{c}$ (Days) & $\boldsymbol{p}$ & $\boldsymbol{\alpha}$ & QOF \\
\hline $\mathbf{2 . 3}$ & 162 & 0.0047 & 0.26 & 0.24 & 1.06 & 0.76 & 0.072 \\
\hline
\end{tabular}

A few of the parameters may offer some potential insight into the seismic characteristics of the RMHC, particularly when compared with the results of the NND analysis. The aftershock productivity $K$ is fairly low for a seismically active area, which agrees with the low number of observed aftershocks and aftershock sequences detected above. This may also be seen in the shallow sloping of the GR distribution (Figure 4-2), which indicates a greater proportion of larger magnitude events and correspondingly fewer smaller magnitude events. The decay parameter $p$ aligns with generally observed values, while the magnitude-weighting parameter $\alpha$ is normal-to-large. This indicates a dependence on event magnitude in the generation of aftershocks, which also coincides with the NND results, as 5 of the 6 significant detected sequences were initiated by a comparatively large order event (M3.4+). The absolute value of the background rate parameter $\mu$ is necessarily non-zero but otherwise unremarkable. The NND model indicates a dominance of loosely clustered activity and few tightly clustered sequences; the gradual ascent of the cumulative ETAS model with few distinct rate spikes agrees with this observation.

\subsection{Montney Clusters (MC1 \& MC2) - Wastewater Disposal \& Hydraulic Fracturing}

The Montney formation stretches from northeastern British Columbia to northwestern Alberta, trending along the Rocky Mountain foothills. Conventional oil and gas production has occurred in the formation since the 1950s, primarily drawing from reservoirs within the sandstone and dolostone layers. Associated wastewater disposal wells have also been active from the 1960s and are suspected to have triggered two significant earthquake clusters (Horner et al., 1994; 
B.C. Oil and Gas Commission, 2014), one north of the town Fort St. John and the other west of Halfway Ranch (Figure 4-7).

Since the mid-to-late 2000s, with the development of horizontal drilling and HF technology, the Montney trend has attracted significant interest for its tight siltstone and shale gas reserves. By 2014, over 1700 natural gas wells were active in the area, many of which had been drilled horizontally after 2005 along the formation's northwestern margin as well as to the southeast near Dawson Creek. In addition, more than 15 wastewater disposal wells have been drilled since 2005, bringing the formation's total to well over 100 (B.C. Oil and Gas Commission, 2014). Since then, substantial low-to-moderate seismicity has been recorded in the area, due to the augmentation of the local seismic network and possibly the increase in subsurface human activity.

Almost all of the recent induced earthquakes within the Montney have occurred within a 150 $\mathrm{km}$ radius, predominantly along the western flank of the trend near the disturbed belt of the foothills. However, the distribution of events has changed over time as the dominant triggering mechanism shifted from wastewater injection to HF (B.C. Oil and Gas Commission, 2014; Atkinson et al., 2016), embodying the changes observed in the regional distributions of the WCSB (Chapter 3). This area was analyzed over two time periods, 1984-2009 and 2010-2018, when first wastewater disposal operations were linked to two distinct earthquake clusters, then later a combination of disposal and horizontally drilled hydraulic fracturing operations became associated with the more recent clustering near Pink Mountain and Buckinghorse River, as well as near Dawson Creek. Events within a $75 \mathrm{~km}$ radius were studied over the first period (MC1, Figure 4-7) and events within a $150 \mathrm{~km}$ radius were studied over the second period (MC2, Figure 4-13), both from the coordinates $[-121.6,56.5]$ in the analyses below. Figure 4-7 and Figure 4-13 show the event maps for both time periods, whereby the change in the spatial distribution of earthquakes is clear.

\subsubsection{First Active Period (MC1) - Wastewater Disposal (1984-2009)}

No seismicity was detected in this area until 1984 when earthquakes arose north of Fort St. John, near wastewater injection wells that had been operating since the early 1970s (Figure 4-7, eastern cluster). A second cluster began to form in 2003, approximately $40 \mathrm{~km}$ west of the first, 
near the town of Halfway Ranch where wastewater disposal had been occurring since the late 1990s (Figure 4-7, western cluster). Small-to-moderate events, both within these two clusters and sporadically elsewhere including to the south near Brule Mines, continued to transpire as more disposal wells became operational.

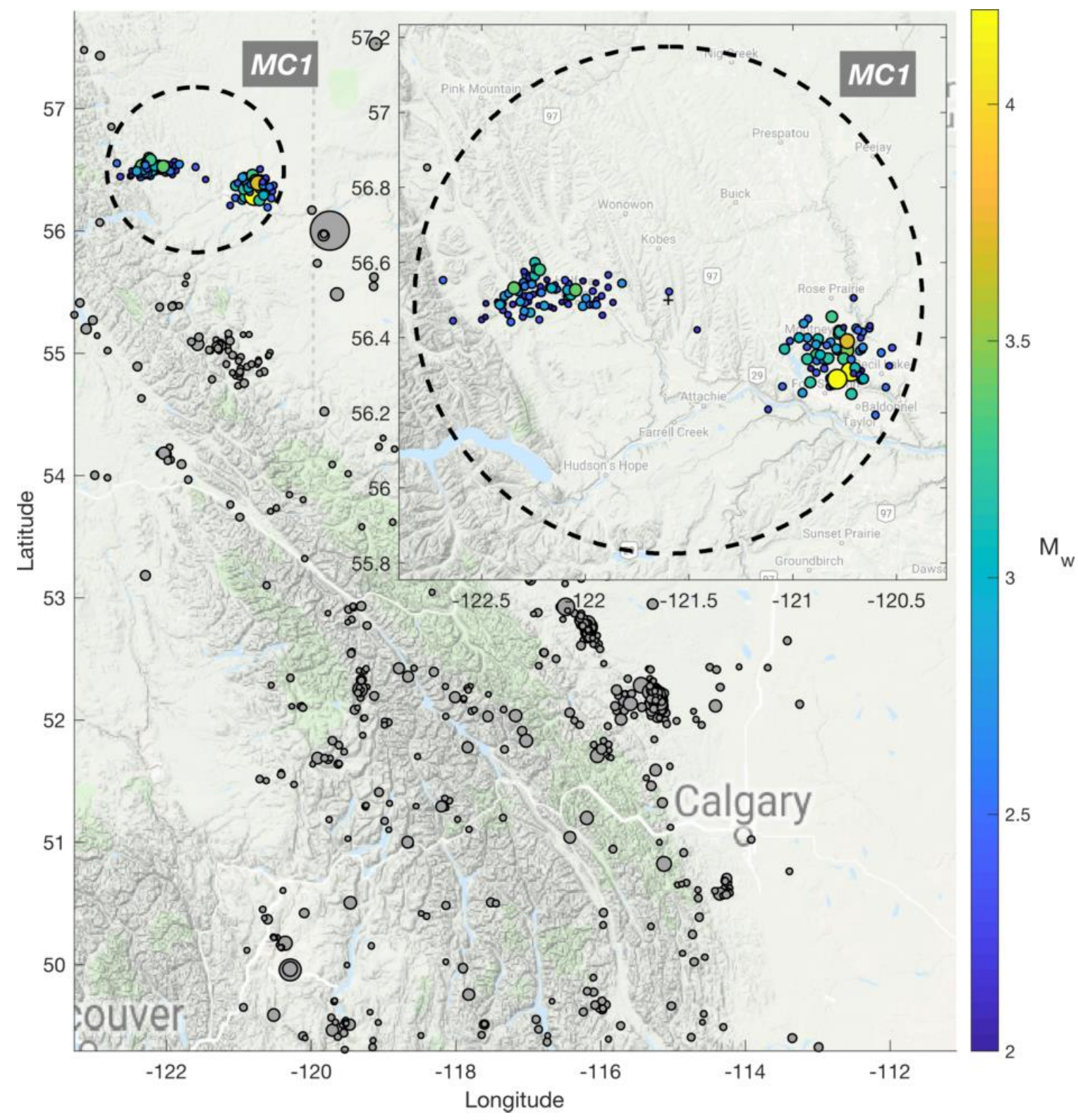

Figure 4-7: Map of the Montney cluster 1 study area between 1984-2009. Dashed circle represents a $75 \mathrm{~km}$ radius from the coordinates [-121.6, 56.5]. Markers are seismic events. Coloured markers are the data points used for analysis. 
Figure 4-8 shows the results of the $M_{c}$ and GR analyses for the dataset. The non-cumulative histogram peaks earlier along the magnitude range at $M=2.3$ (panel a). For almost the entire range, as the cut-off magnitude increases so do the $b$-values (panel c). A brief plateau occurs between $2.7 \leq M_{0} \leq 2.9$ and the rolling average $b_{\text {ave }}$ (orange line with triangle markers) stabilizes in this region, though uncertainties (dashed black lines) are large. The GFT computes a best-fitting synthetic FMD at $M=2.7$.

Visual inspection of the MBS results agrees with the residual minimization of the GFT, while the MAXC method appears to underestimate completeness (i.e. the $b$-values surrounding the determined MAXC value are unstable). The GR parameters were computed using $M_{c}=2.7$; the $b$-value is approximately 1 , which suggests a more typical distribution of event magnitudes. However, the high completeness value results in a smaller data sample and thus larger uncertainties (Figure 4-8, panel d; Table 4-5). The 95\% confidence intervals indicate that the true $b$-value exists between 0.8 and 1.28 , a range too large to derive any significant conclusions regarding the scaling of event magnitudes within the MC1. 
a)

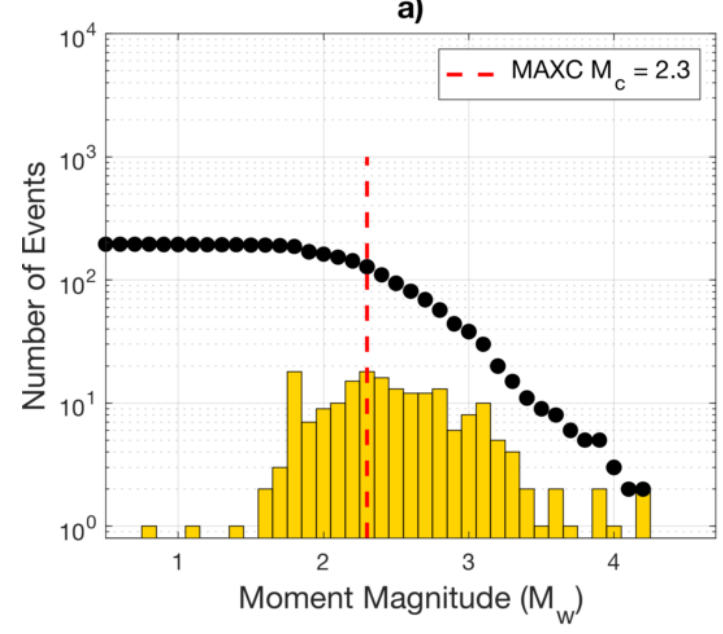

c)

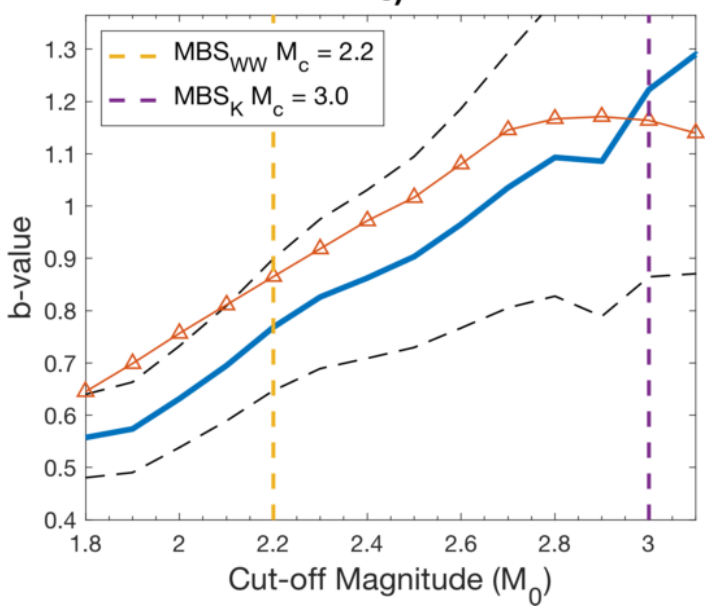

b)

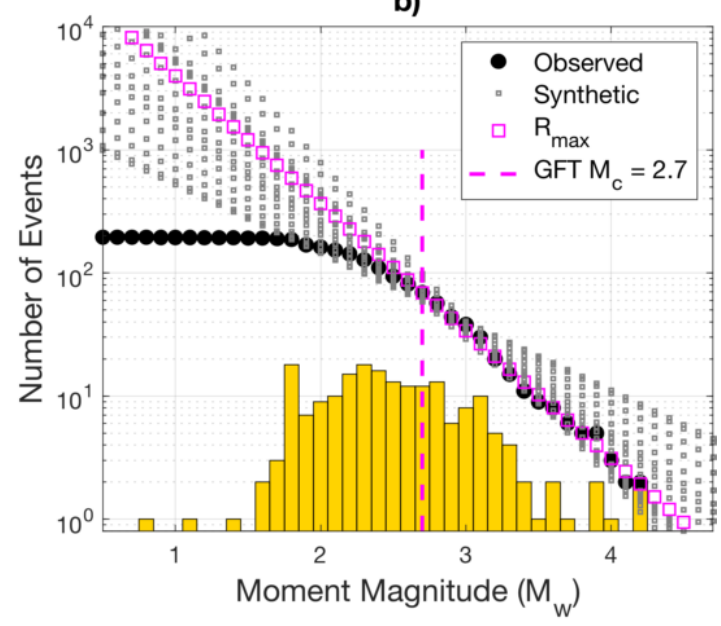

d)

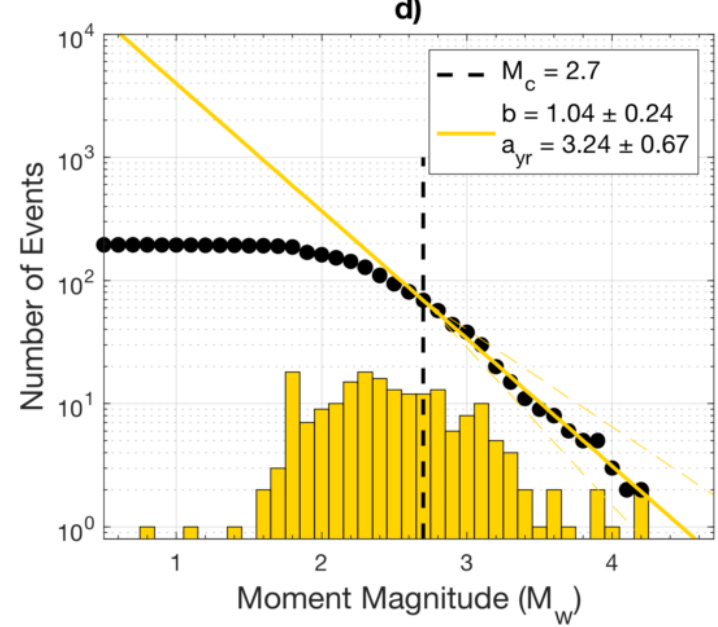

Figure 4-8: $M_{c}$ and GR Analyses for the Montney cluster 1. a) Maximum Curvature Method (MAXC). b) Goodness-of-Fit Test (GFT). c) Method of $b$-value Stability (MBS). d) GR parameter estimation using determined $M_{c}$.

Table 4-5: Summary of $M_{c}$ and GR Analyses for the Montney cluster 1.

\section{$\begin{array}{llllllll}\text { N } & \text { MAXC } & \text { GFT } & \text { MBS }_{W w} & \text { MBSK }_{K} & \text { Determined } M_{c} & b & a\end{array}$}

$\begin{array}{llllllll}314 & 2.3 & 2.7 & 2.2 & 3.0 & 2.7 & 1.04 \pm 0.24 & 3.24 \pm 0.67\end{array}$

Figure 4-9 gives the NND model results using the determined $b$-value of 1.04. Similar to the RMHC, there is a clear dominance of middle-mode, loosely clustered background events and a smaller proportion of tightly clustered events. Also worth noting is the broad distribution of 
tightly clustered events, which extend to extremely small inter-event distances. It is demonstrated below that this is a consequence of location-determination inaccuracy by the network, which mistakenly places several event family members in the exact same spot.

a)

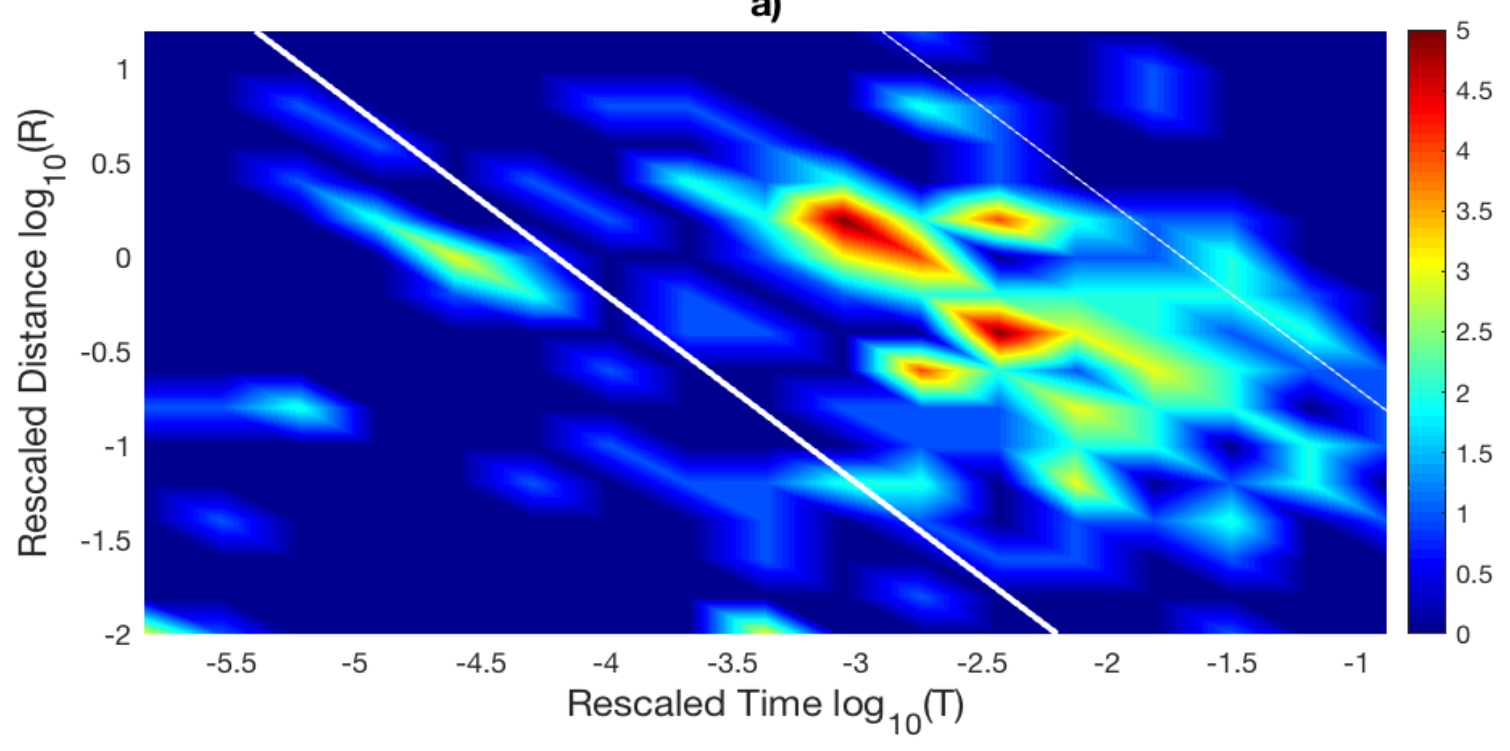

b)

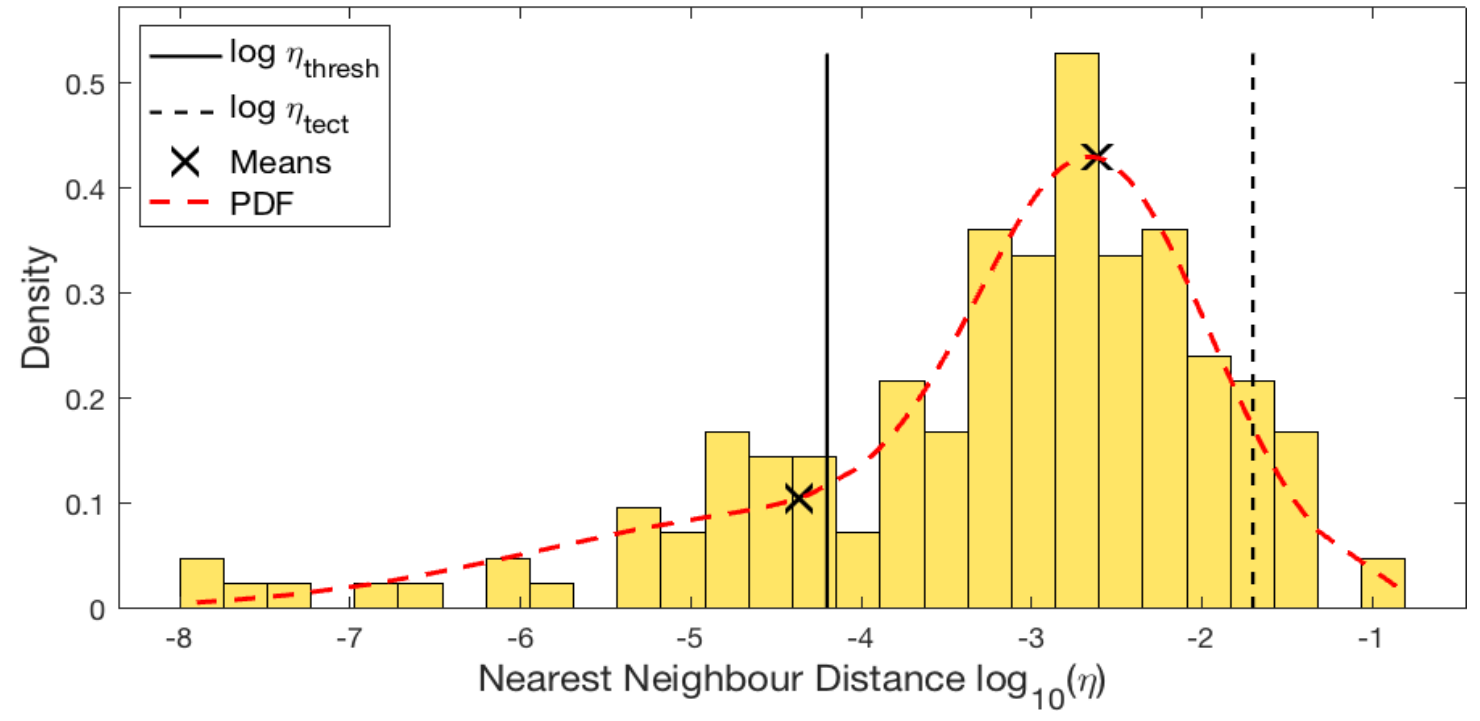

Figure 4-9: Nearest-Neighbour Distance distribution for the Montney cluster 1. a) Joint distribution of the temporal and spatial components $(T, R)$. b) Normalized density of $\eta$ values. 
Table 4-6: Event Family Breakdown for the Montney cluster 1.

\begin{tabular}{ccccc}
\hline Singles & Foreshocks & Mainshocks & Aftershocks & Aftershocks of Aftershocks \\
\hline $105(64.8 \%)$ & $13(8.0 \%)$ & $22(13.6 \%)$ & $22(13.6 \%)$ & $2(1.2 \%)$ \\
\hline
\end{tabular}

As suggested by Figure 4-9, the MC1 is largely composed of loosely clustered singles (Table 4-6). Of the 127 total background events, 22 triggered a second event and only 2 eventually triggered a higher order event. There are few foreshocks and almost no higher order offspring events. It is interesting to note that, though a substantial number of earthquakes occurred over a short time interval between 2004-2006 (Figure 4-10) within the more recent cluster near Halfway Ranch, few event sequences transpired. This may be attributable to the low-magnitude nature of the second cluster, where most events occurring were of M2.7 or lower. The majority, though not all, of the mainshocks identified in either cluster were of $\mathbf{M} 3$ or greater. The two largest sequences detected by the NND algorithm, both comprising of an M4+ mainshock, persist over longer stretches of time while the rest of the families decay rapidly (discussed below). 


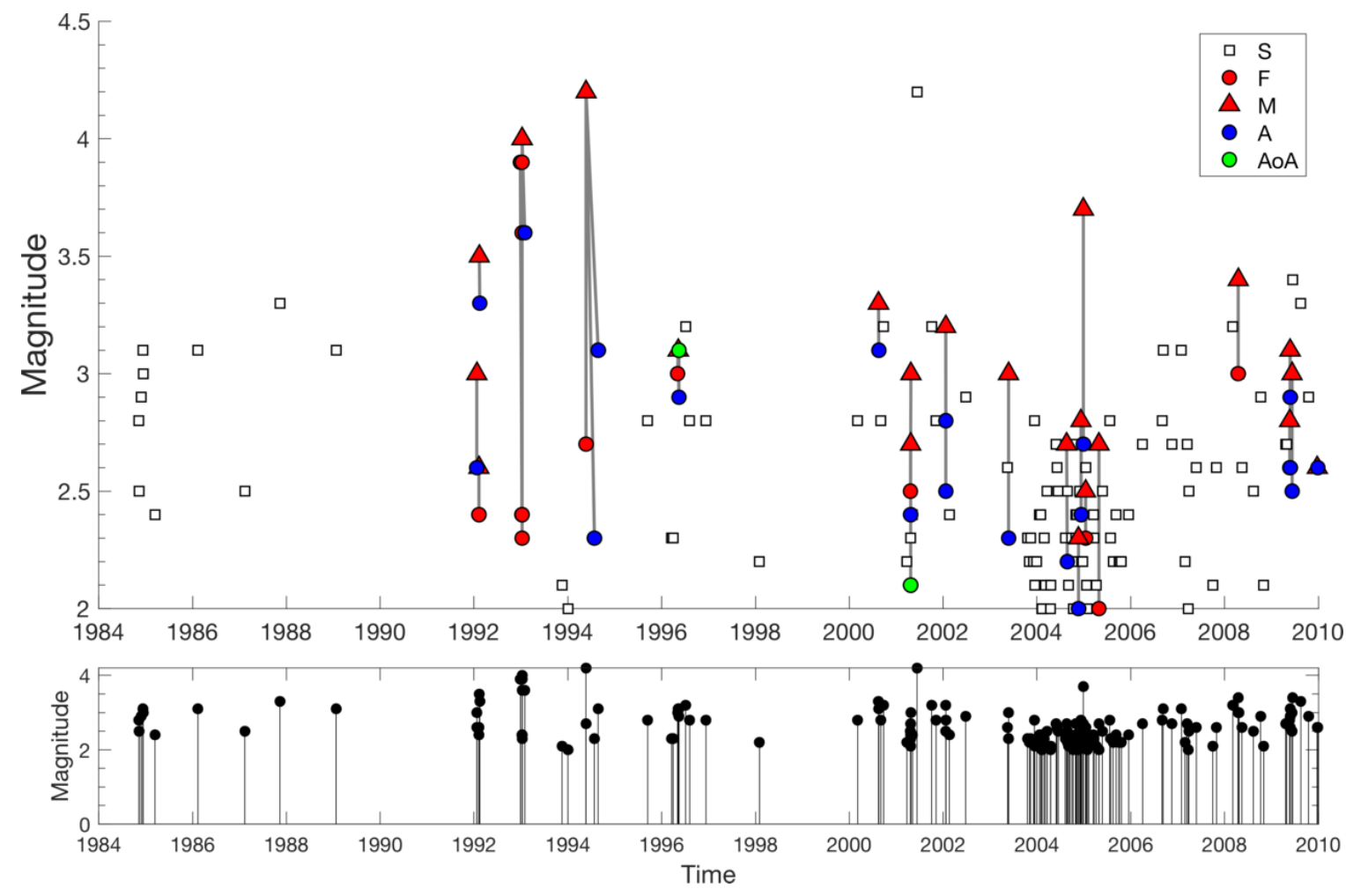

Figure 4-10: Magnitude-Time evolution of event families of the Montney cluster 1. Lower plot: Event magnitudes vs. time. Upper plot: Event-type classification over time. White squares are singles, red circles are foreshocks, red triangles are mainshocks, blue circles are aftershocks, and green circles are aftershocks-of-aftershocks. Grey lines represent the strong links connecting individual event families.

As mentioned, several of the recorded events suffered from location inaccuracies, where the network placed them in the same geographic location. It is possible that this created erroneous connections between events when the NND model was applied. However, the events in question also occurred closely together in time (within the span of a month) and so it is reasonable to assume that, within the local network uncertainty, most events that were identified as part of a sequence likely would have been linked together anyway. Though the spatial maps for these particular sequences are inconclusive, the temporal and topological structures manage to give clearer results. The event family configurations are summarized in Table 4-7 and plotted in Figure 4-11. The two largest families are moderate aftershock 
sequences, which both contain M4+ mainshocks and continue far longer than the other sequences. The largest family contains 8 events, all placed in the same location. The magnitude-time and topological plots display an image of a smaller burst triggering a larger one, with a low normalized leaf depth and low inverted branching number. The second sequence is visually a burst, where a single foreshock led to a large-order mainshock and two subsequent aftershocks, the second of which occurred a month after the first. The third sequence appears path-like in time, but event locations were again improperly recorded. Due to the similarity in magnitudes, higher normalized leaf depth and chain-like temporal structure (high inverted branching number), it is classified as a swarm. The fourth event family is a smaller swarm that decays rapidly while the fifth and sixth are small bursts.

a)
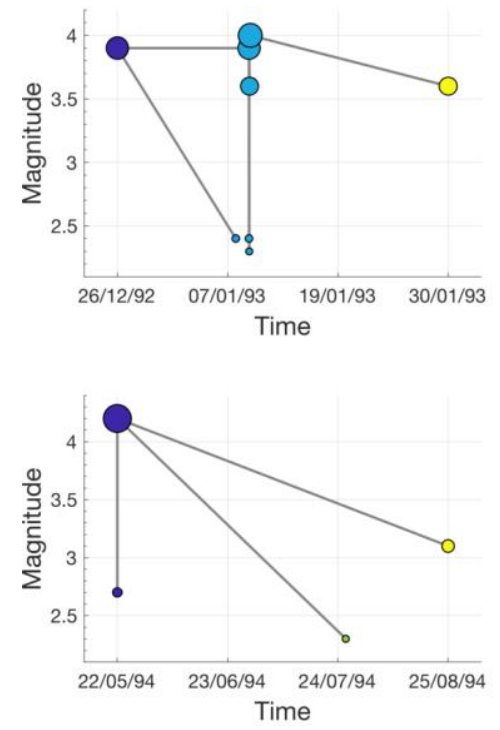

b)

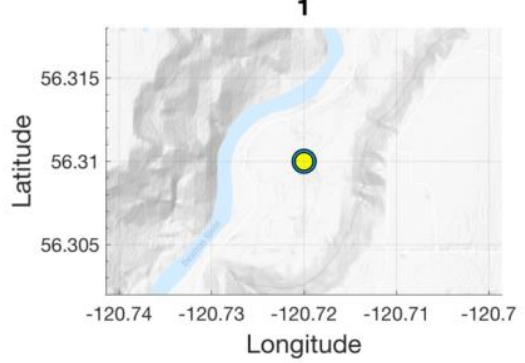

2

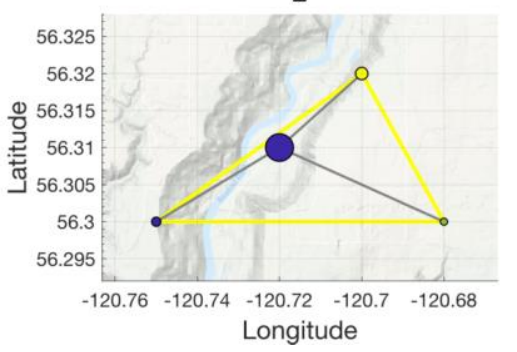

c)
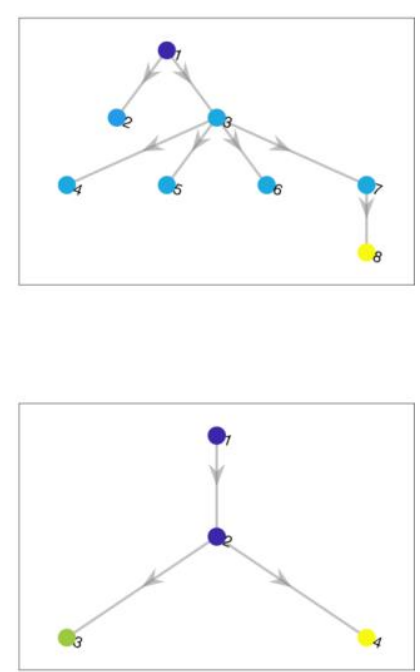

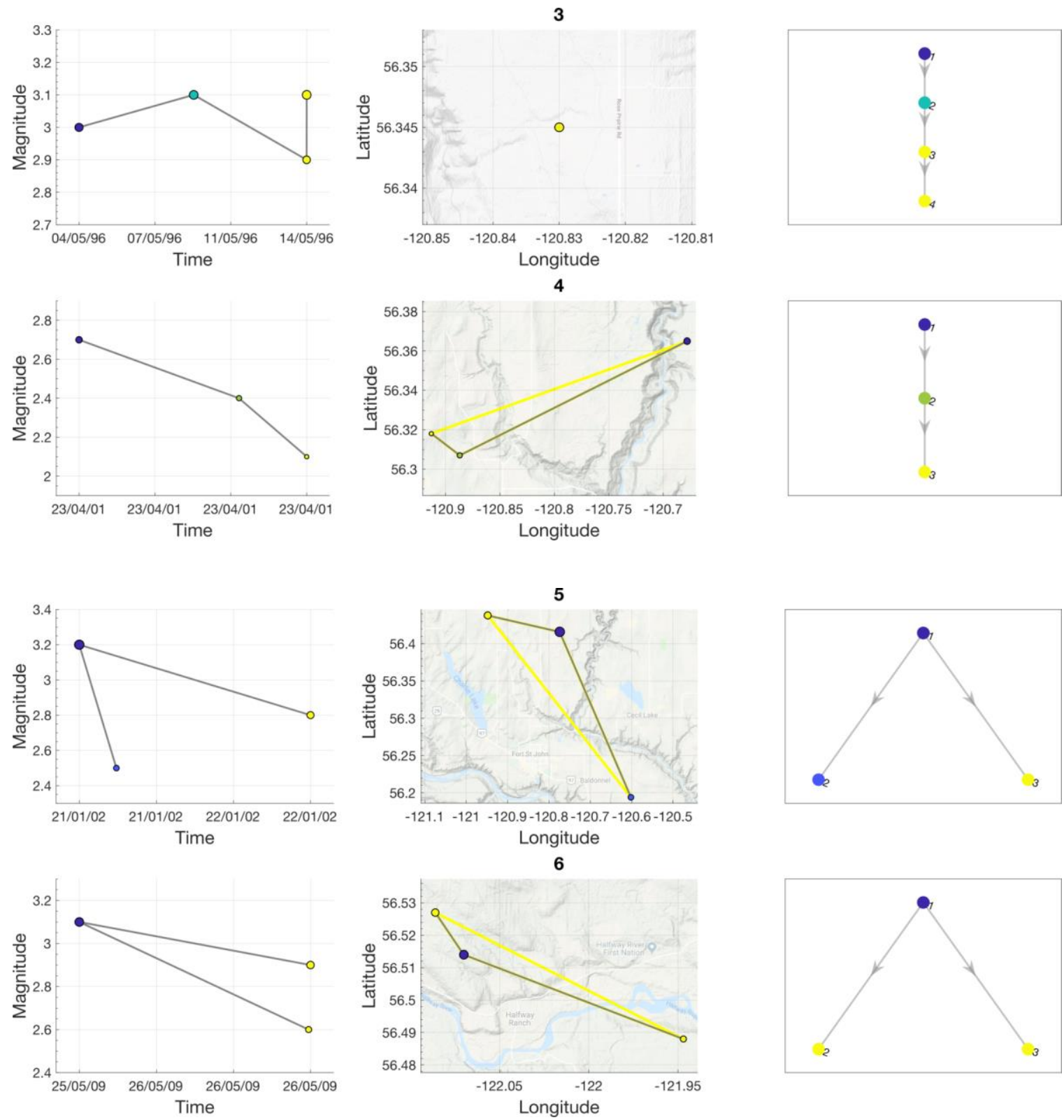

Figure 4-11: Event Family Structures within the Montney cluster 1. a) Moment magnitude vs. Time in days. b) Spatial map. Yellow border outlines the hull area occupied by the sequence. c) Directed tree graph in dimensionless space. Data points are coloured chronologically from darkest to lightest. 
Table 4-7: Parameter sets for event families within the Montney cluster $1 . \mathrm{N}$ is the size of the sequence, $\langle d\rangle$ is the average leaf depth, $\delta$ is the normalized leaf depth, $B_{i}$ is the inverted branching number, $A$ is the spatial area, $t_{d}$ is the decay period, and $\Delta M$ is the magnitude differential.

\begin{tabular}{cccccccccc}
\hline Sequence & $\mathbf{N}$ & $\langle\boldsymbol{d}\rangle$ & $\boldsymbol{\delta}$ & $\boldsymbol{B}_{\boldsymbol{i}}$ & $\boldsymbol{A}\left(\mathbf{k m}^{2}\right)$ & $\boldsymbol{t}_{\boldsymbol{d}}$ (days) & $\boldsymbol{\Delta M}$ & Class \\
\hline $\mathbf{1}$ & 8 & 2.00 & 0.71 & 0.43 & 0.00 & 34.88 & 0.10 & Burst \\
$\mathbf{2}$ & 4 & 2.00 & 1.00 & 0.67 & 4.80 & 94.82 & 1.10 & Burst \\
$\mathbf{3}$ & 4 & 3.00 & 1.50 & 1.00 & 0.00 & 10.14 & 0.00 & Swarm \\
$\mathbf{4}$ & 3 & 2.00 & 1.15 & 1.00 & 13.06 & 0.04 & 0.30 & Swarm \\
$\mathbf{5}$ & 3 & 1.00 & 0.58 & 0.50 & 118.78 & 0.80 & 0.40 & Burst \\
$\mathbf{6}$ & 3 & 1.00 & 0.58 & 0.50 & 4.04 & 0.90 & 0.20 & Burst \\
\hline
\end{tabular}

The ETAS model results using events above or equal to $M_{c}=2.7$ are plotted in Figure 4-12 and summarized in Table 4-8. 69 events were used in the analysis, which is at the small end of appropriate datasets. The model fit is fair, with a QOF of 0.042 , and manages to capture some of the trends occurring in the rate. The latter half of the time period is over-predicted, as can be seen in original and transformed times where the observed cumulative count sits slightly beneath the modeled plot. 
a)

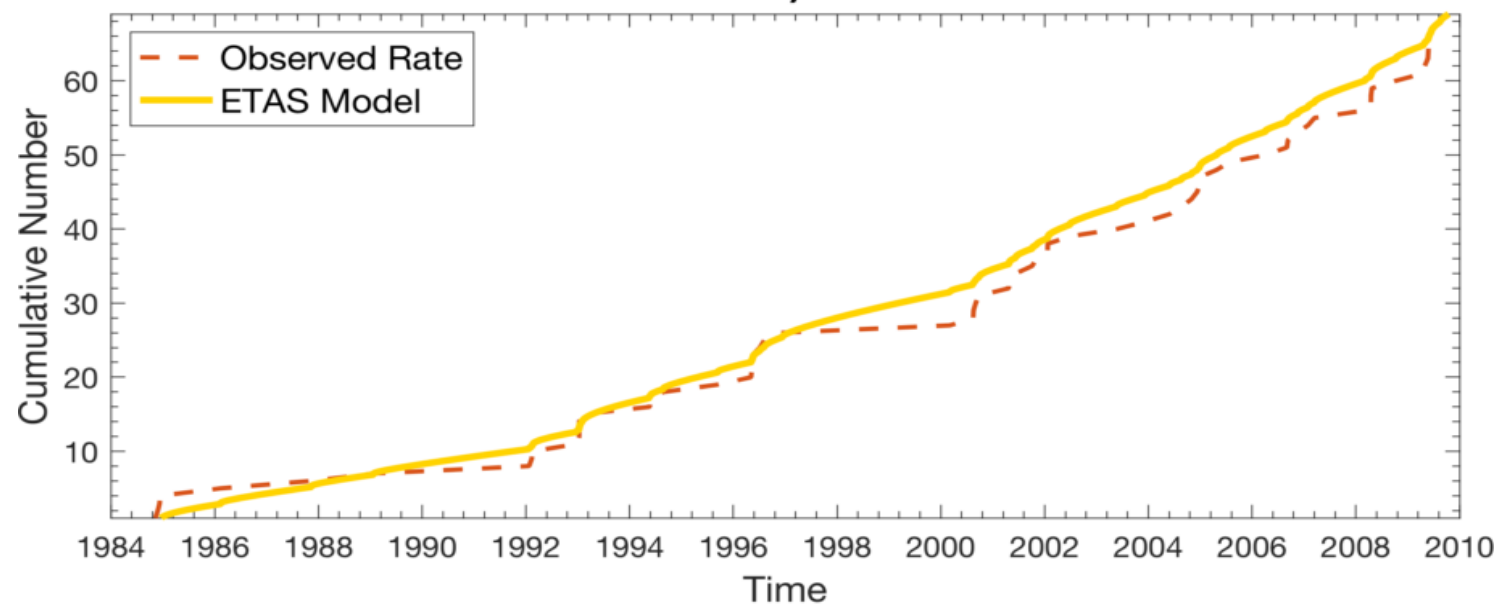

b)
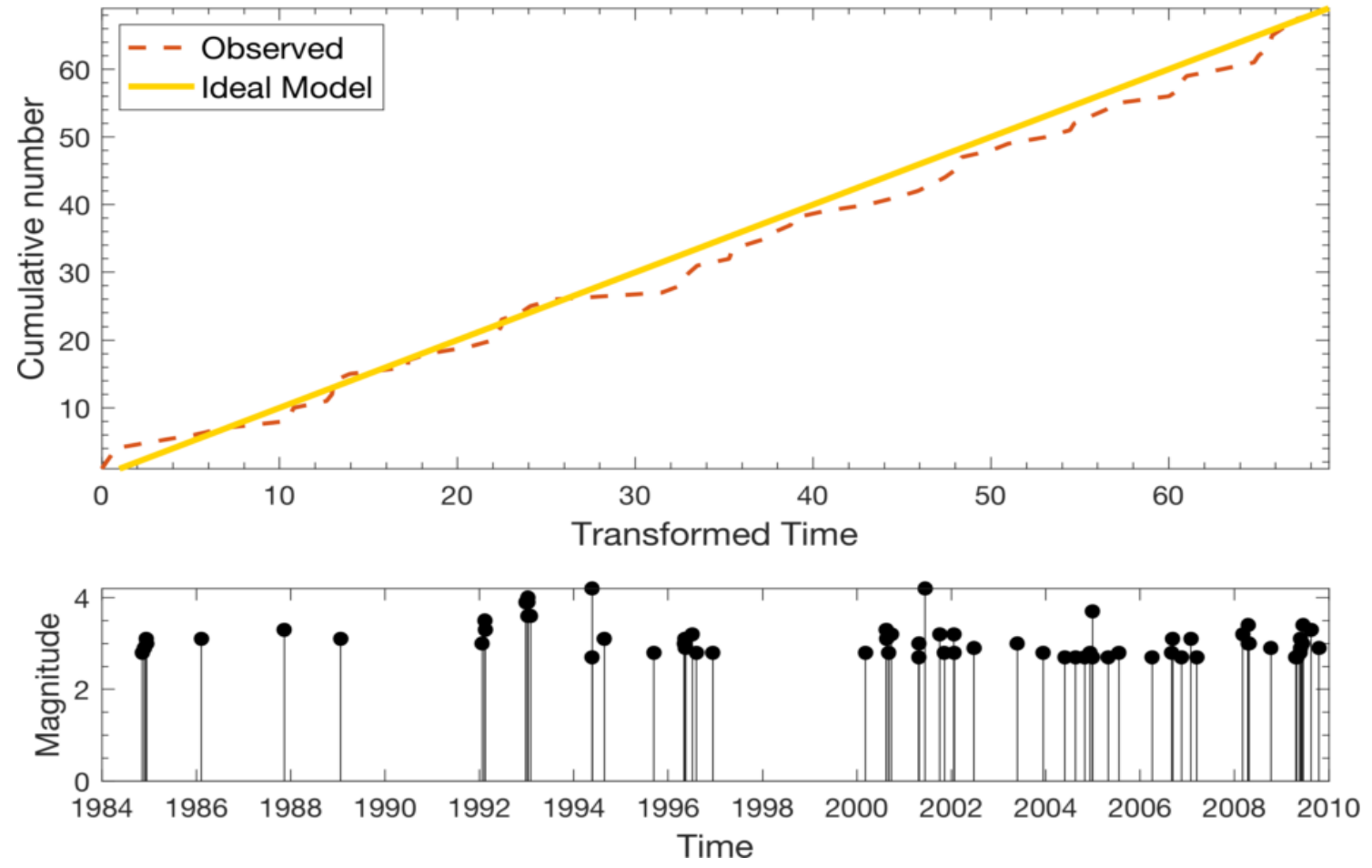

Figure 4-12: ETAS Model of the Montney cluster 1 using $M_{c}=2$. 7. a) Original time plot.

Dashed orange line is the observed cumulative rate of seismicity. Solid line is the optimized model. b) Transformed time plot. Solid line represents the "perfect" model fit. 
Table 4-8: ETAS model parameters for the Montney cluster $1 . \mathrm{N}$ is the number of events modeled, $\mu$ is the constant background rate, $K$ is the aftershock productivity, $c$ is the temporal offset, $p$ is the exponential decay, $\alpha$ is the magnitude-aftershock dependence, and QOF is the quality of the model fit.

\begin{tabular}{cccccccc}
$M_{\boldsymbol{c}}$ & $\mathbf{N}$ & $\boldsymbol{\mu}$ (Events/Day) & $\boldsymbol{K}$ (Events/Day) & $\boldsymbol{c}$ (Days) & $\boldsymbol{p}$ & $\boldsymbol{\alpha}$ & $\mathbf{Q O F}$ \\
\hline 2.7 & 69 & 0.0033 & 3.09 & 0.0019 & 0.81 & 0.84 & 0.042 \\
\hline
\end{tabular}

The decay exponent $p$ is lower than average, possibly indicating a slower aftershock decay rate. This correlates with the two largest NND sequences identified above, which persist for a month or longer after the root event, but disagrees with the remainder of the sequences. A low $p$ parameter may also be a consequence of unrelated background events being grouped together with genuine sequences, as the ETAS model used in this study assumes spatial independence with respect to the rate. In other words, any group of events within a given dataset that happen to occur closely together in time will contribute to a spike in the temporal earthquake rate regardless of their spatial relationship. It is difficult to assess to what degree this affects the parameter results, if at all, and is something worth studying further. The $\alpha$ value is moderate-tolarge, indicating a dependence on magnitude in the generation of aftershocks. This potentially agrees with the NND analysis, as 4 of the 6 event families are bursts and all contain M3+ mainshocks (with the two largest containing M4+ mainshocks).

\subsubsection{Second Active Period (MC2) - Wastewater Disposal \& Hydraulic Fracturing (2010-2018)}

After 2009, seismicity began to arise away from the two original clusters, as multistage HF operations increasingly populated the area. Additionally, by August 2013, six more seismic stations were added to the local detection network and the number of smaller magnitude events recorded across the trend increased (B.C. Oil and Gas Commission, 2014). The spatial distribution of seismicity changed markedly between time frames; a new set of clusters can be observed along the northwestern margin of the Montney formation, spanning from Buckinghorse River south to Hudson's Hope, overlapping the existing cluster near Halfway 
Ranch. Another extended cluster appears south of Fort St. John and stretches east towards Dawson Creek (Figure 4-13).

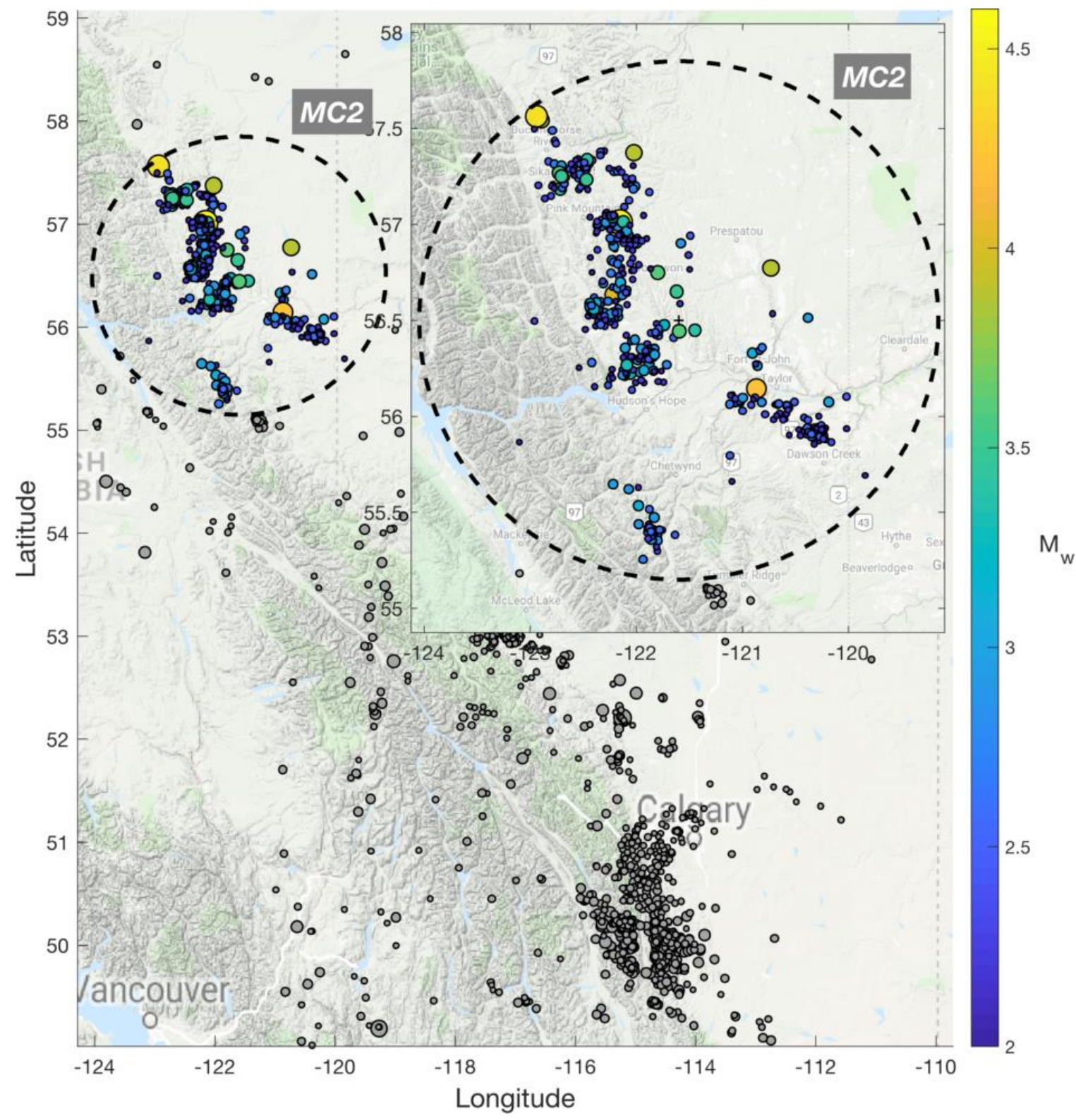

Figure 4-13: Map of the Montney cluster 2 study area between 2010-2018. Dashed circle represents a $150 \mathrm{~km}$ radius from the coordinates [-121.6, 56.5]. Markers are seismic events. Coloured markers are the data points used for analysis. 
The results of the $M_{c}$ and GR analyses for the MC2 are shown in Figure 4-14 and summarized in Table 4-9. From the FMD, it is immediately obvious that many more small magnitude events were recorded over this time interval in comparison with the previous period. This is likely due to the improvement in network coverage, but may also be partially a consequence of the change in triggering mechanisms. The completeness magnitude does not appear to reduce drastically despite the large number of low-magnitude earthquakes in the dataset, though the noncumulative FMD peaks very early at $M=1.8$ (Figure 4-14, panel a). The GFT computes a best-fitting synthetic distribution at $M=2.6$; the ideal distribution fits well (panel b, pink squares) but perhaps very slightly underestimates the FMD at large magnitudes. In terms of $b$ value stability, the rolling average $b_{\text {ave }}$ dips below uncertainty at $M_{0}=2.1$ (panel c, orange line with triangle markers), but the actual $b$-values only begin to stabilize near $M_{0}=2.4$ (panel c, blue line). The rolling average also stabilizes in the region $2.4 \leq M_{0} \leq 2.7$.

Once again, the GFT and MBS estimates fall within close proximity, while the MAXC method likely under-predicts $M_{c}$. The GR parameters were computed using $M_{c}=2.5$; both the $b$-value and $b_{\text {ave }}$ are stable at this point and it appears that the resulting GR distribution properly accounts for the largest magnitudes. There is a slight increase in the determined $b$-value for this dataset, indicating a rise in the proportion of smaller-magnitude events in comparison with the previous time period (MC1); however, the magnitude of the increase is well within uncertainties. The normalized $a$-value is larger as well, indicative of the overall growth in the detected seismicity rate. 
a)

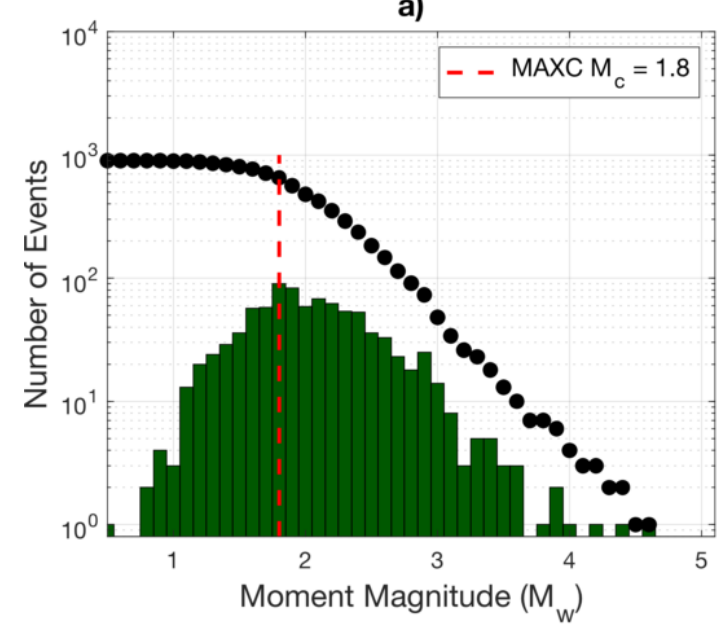

c)

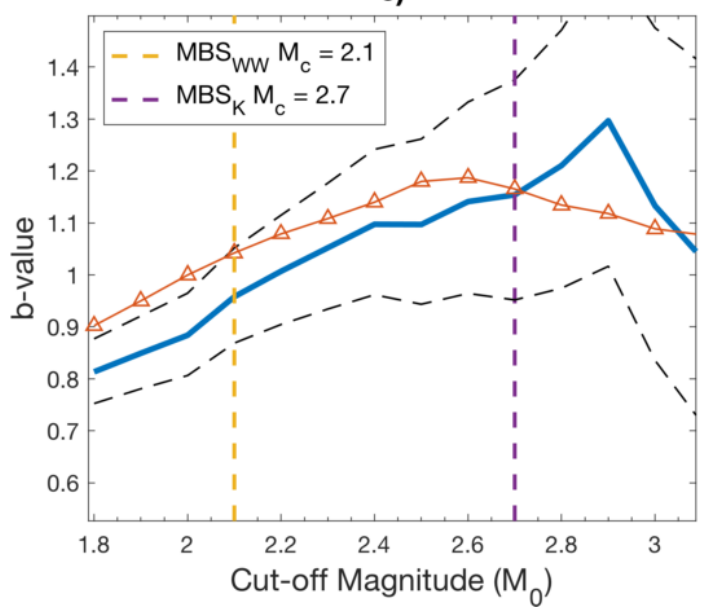

b)

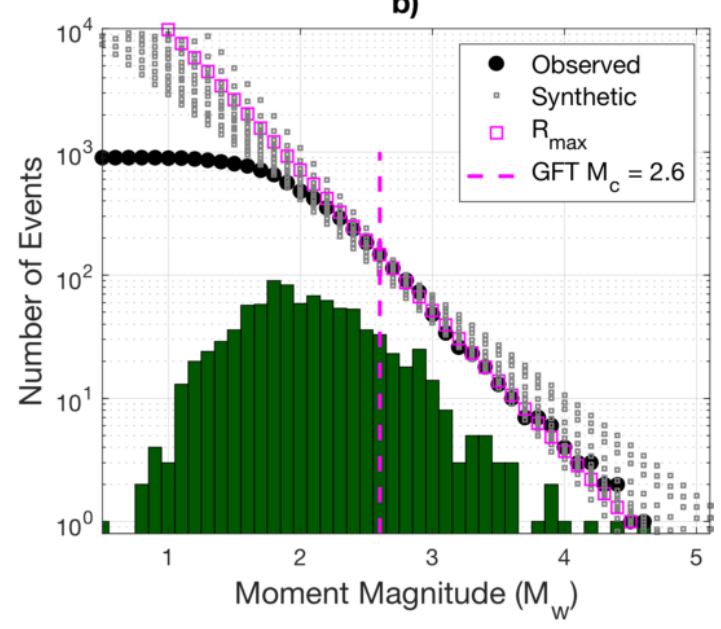

d)

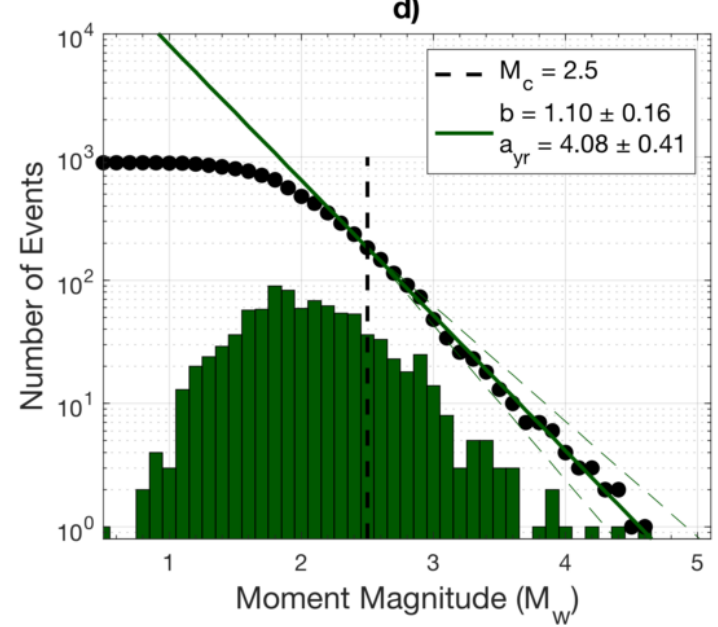

Figure 4-14: $M_{c}$ and GR Analyses for the Montney cluster 2. a) Maximum Curvature Method (MAXC). b) Goodness-of-Fit Test (GFT). c) Method of $b$-value Stability (MBS). d) GR parameter estimation using determined $M_{c}$.

Table 4-9: Summary of $M_{c}$ and GR Analyses for the Montney cluster 2.

N MAXC GFT MBSww MBSк Determined $M_{c} \quad b \quad a$

$\begin{array}{llllllll}900 & 1.8 & 2.6 & 2.1 & 2.7 & 2.5 & 1.10 \pm 0.16 & 4.08 \pm 0.41\end{array}$

The resulting NND distribution for the MC2 is plotted in Figure 4-15. Similar to the transformations occurring at the regional level (Chapter 3.2), the distribution of inter-event distances changes distinctly over this time frame. A clustered mode appears, which potentially 
signifies an increase in tightly grouped seismic activity. Again, this could simply be a consequence of the augmented network, which allowed for the discovery of previously undetectable clustered seismicity occurring at lower magnitude levels. In theory, if the observed increases in tightly clustered activity across time were due solely to improvements in detection capability, then the rates of detected seismicity within the other modes would have increased proportionally. Though there is a substantial increase in the number of detected events during this time period, the distribution across subpopulations is not proportionate. While the yearlydetected occurrence rate of the deep background increases by a factor of 6.8 and the loosely clustered background rate increases by a factor of 7.4, the tightly clustered rate increases by a factor of 13.4, nearly double that of the other two modes. This justification assumes that the NND earthquake subpopulations are similarly distributed across magnitudes. As is observed below and in the regional FMDs of the separated modes in Chapter 3 (Figure 3-5), both the tightly and loosely clustered subpopulations have a higher tendency to manifest at lower magnitudes (they have higher $b$-values) than do the more typically distributed deep-background events (with $b$-values near unity). The disproportionate rate changes occurring between the tightly and loosely clustered modes from MC1 to MC2 may therefore be significant, as they are similarly distributed in their magnitude occurrence-frequency and thus more comparable. This lends credibility to another observation made in Chapter 3, which stated that loosely clustered activity may be a common feature among discrete cases of induced seismicity across space and time (conventional production, wastewater disposal and HF), while the recent tightly clustered activity may be a more singular consequence of the surge in extended HF application. It seems possible that the increase in detected clustered activity is partially correlated with the rise in unconventional resource development across the Montney trend, but is also a byproduct of improved monitoring. 
a)

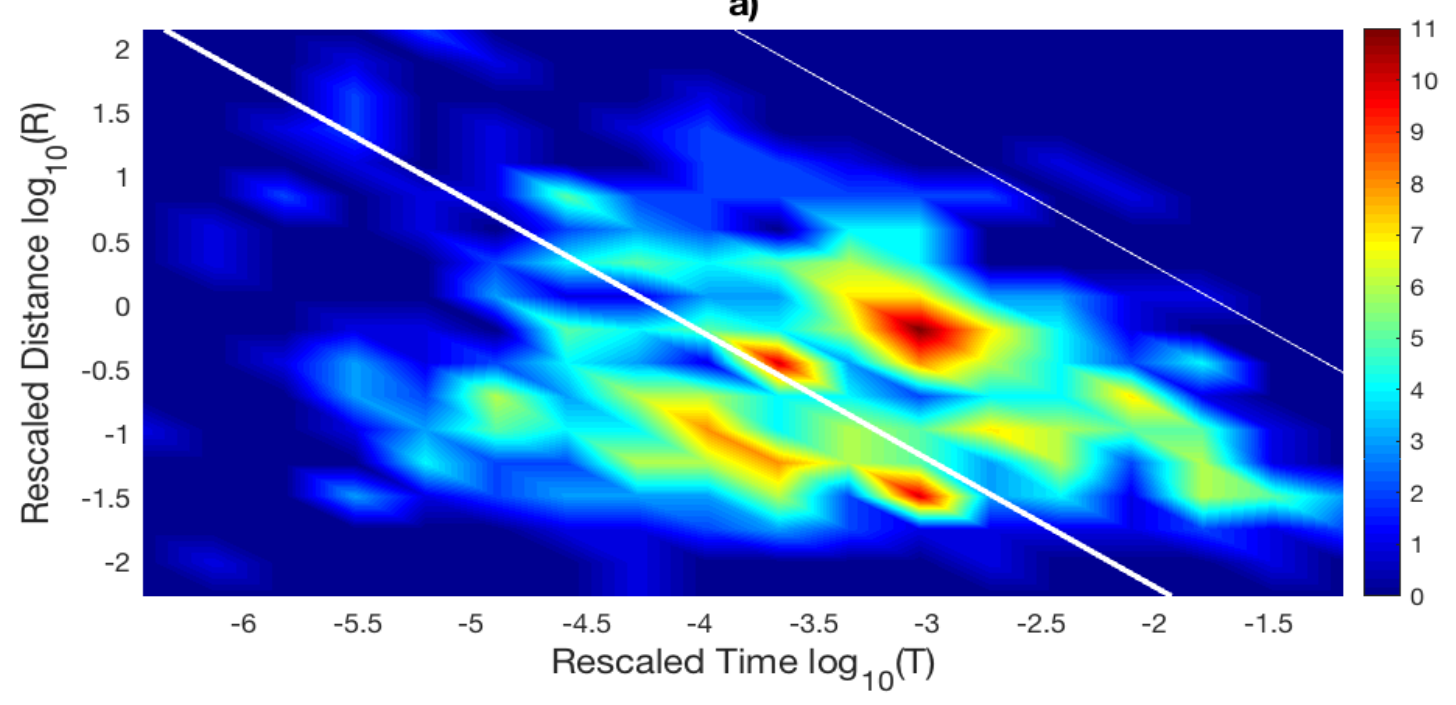

b)

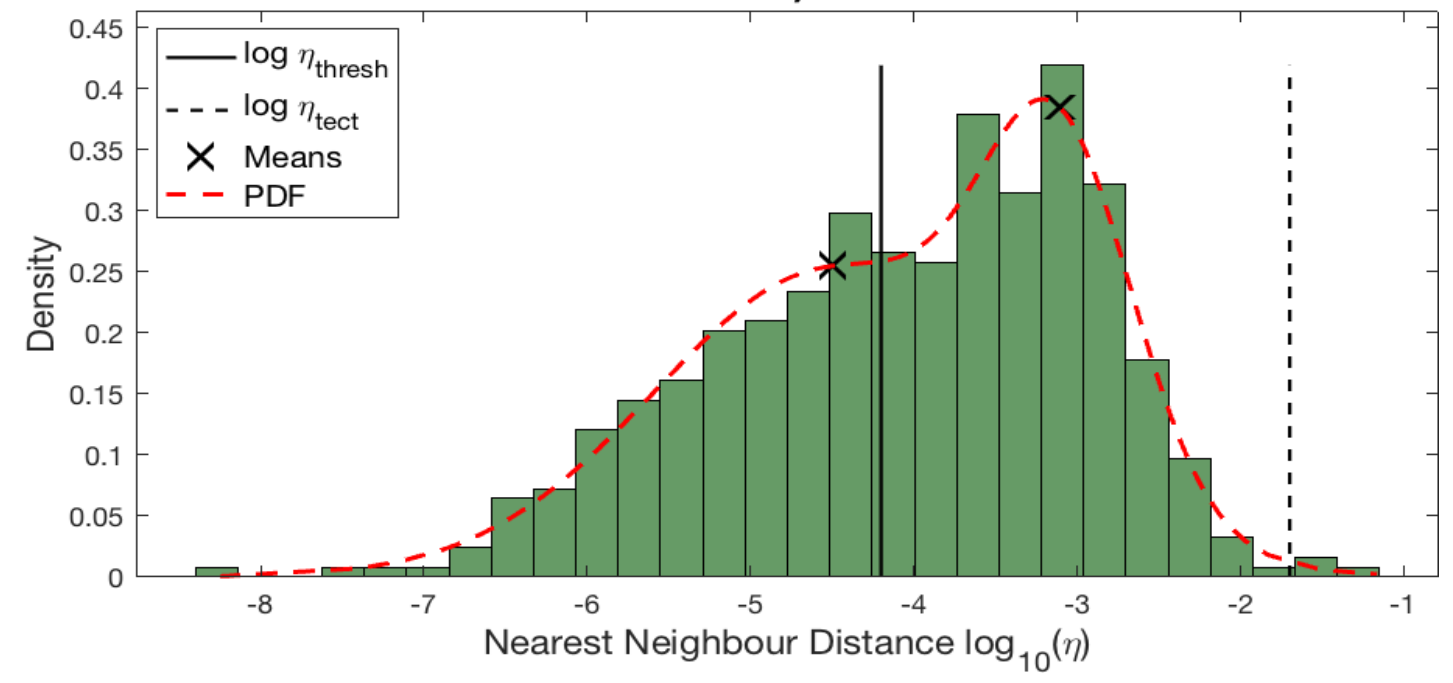

Figure 4-15: Nearest-Neighbour Distance distribution for the Montney cluster 2. a) Joint distribution of the temporal and spatial components $(T, R)$. b) Normalized density of $\eta$ values.

Table 4-10: Event Family Breakdown for the Montney cluster 2.

Singles Foreshocks Mainshocks Aftershocks Aftershocks of Aftershocks

$257(53.7 \%) \quad 37(7.7 \%) \quad 69(14.4 \%) \quad 116(24.2 \%) \quad 41(8.6 \%)$


The effect of the added seismic stations is apparent in Figure 4-16, as many more lowmagnitude events are observed after 2013. A small majority of the total events are singles (54\%), which is an $11 \%$ decrease from the previous period, and hence more families are identified overall within MC2 (69). The family structures appear bigger as well as deeper in their topology; this is shown by the large jumps in aftershock and higher-order-aftershock numbers (Table 4-10). From Figure 4-16, it is clear that the majority of both recent loosely clustered singles and tightly clustered family members occur at lower magnitudes.

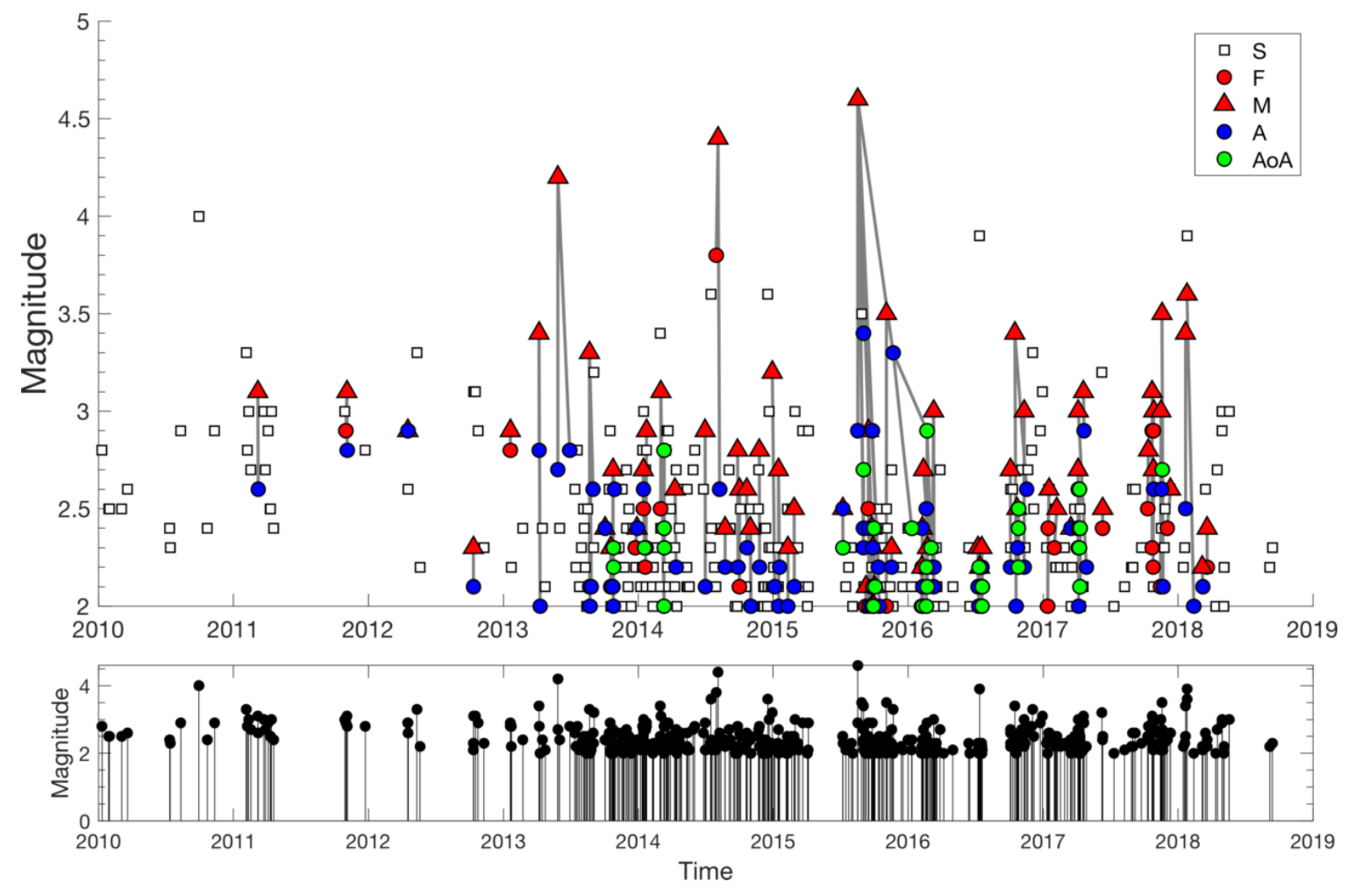

Figure 4-16: Magnitude-Time evolution of event families of the Montney cluster 2. Lower plot: Event magnitudes vs. time. Upper plot: Event-type classification over time. White squares are singles, red circles are foreshocks, red triangles are mainshocks, blue circles are aftershocks, and green circles are aftershocks-of-aftershocks. Grey lines represent the strong links connecting individual event families.

10 distinct event families are identified within this time frame; their temporal, spatial and topological structures are shown in Figure 4-17 and the resulting parameter sets are summarized in Table 4-11. 8 of the sequences are classified as swarms and the remaining 2 are bursts. The 
bursts are easily distinguishable by their spray-like shape and dominant mainshocks (clusters 2 and 6). Cluster 2 contains the largest mainshock in the dataset (M4.7), occupies the largest spatial area $\left(194 \mathrm{~km}^{2}\right)$ and stretches the longest time frame (198.5 days). The swarms adopt many of the prototypical characteristics (fewer leaves, large leaf depths and inverted branching numbers, small magnitude differentials, chain-like appearances over time) and have short decay periods, with a peak life span of 10 days. It is possible that the length of these decay periods may be related to the intensity, scope and/or frequency of their triggering mechanisms, such as the pumping rate or net injected volume of associated HF operations (discussed further in Chapters 4.3 and 4.4).

a)
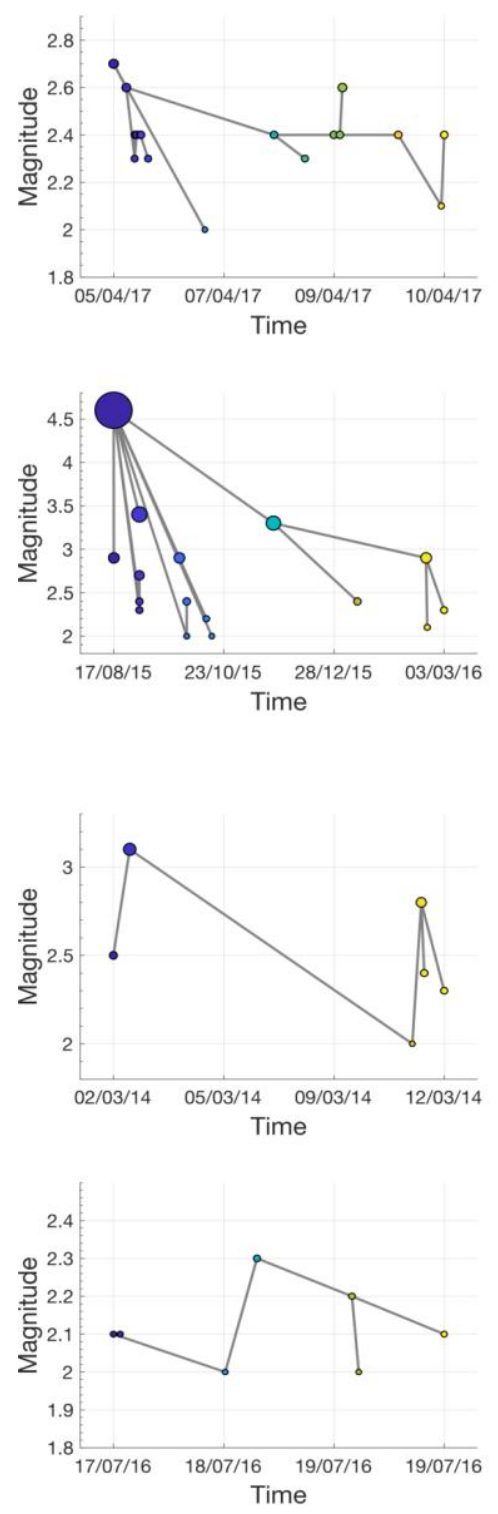

b)

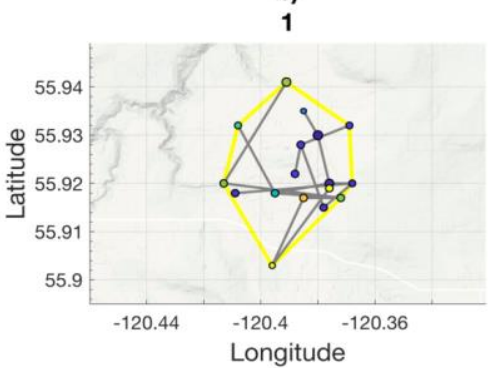

2

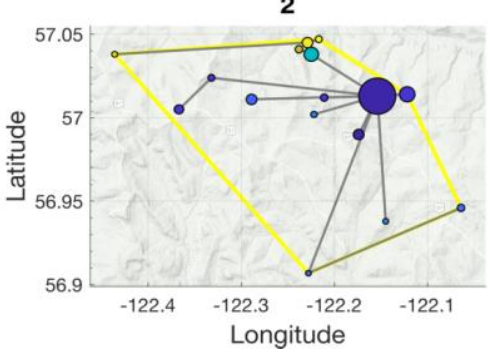

3

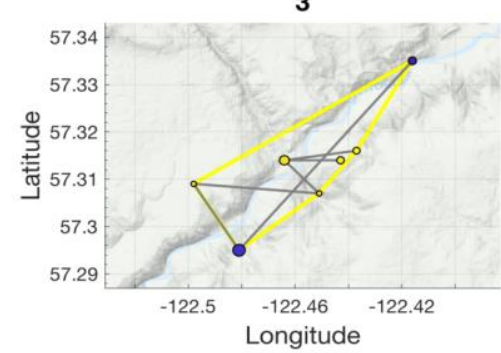

4

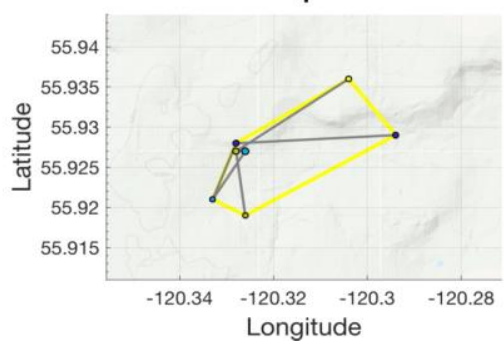

c)
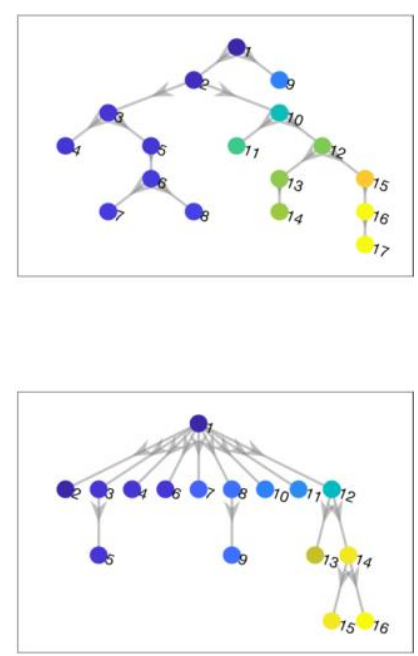
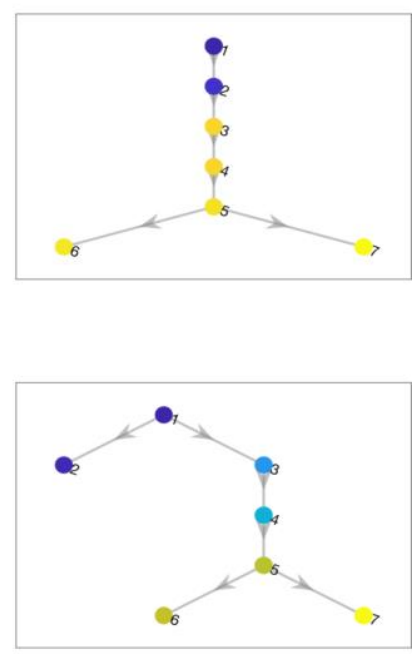

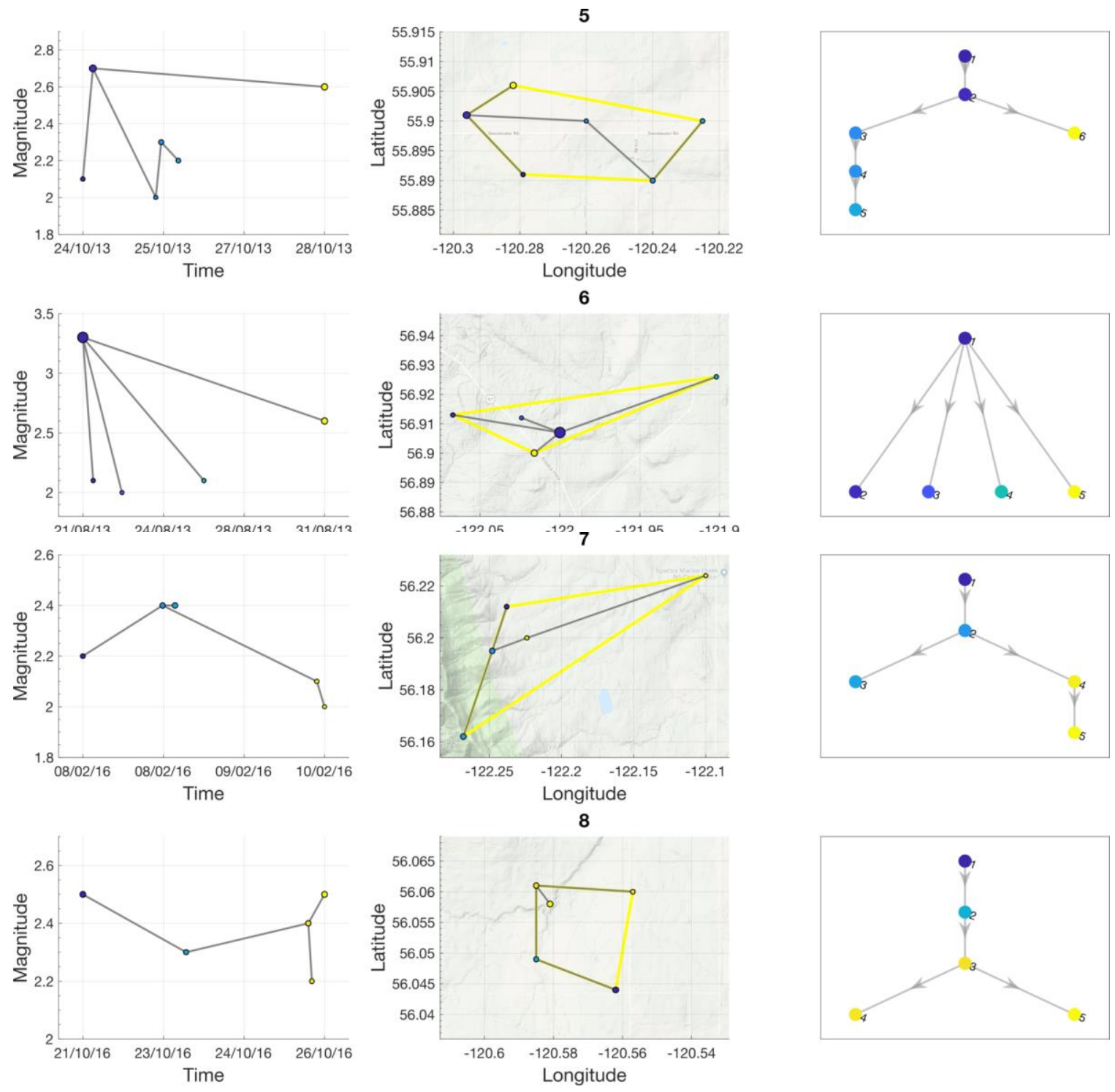

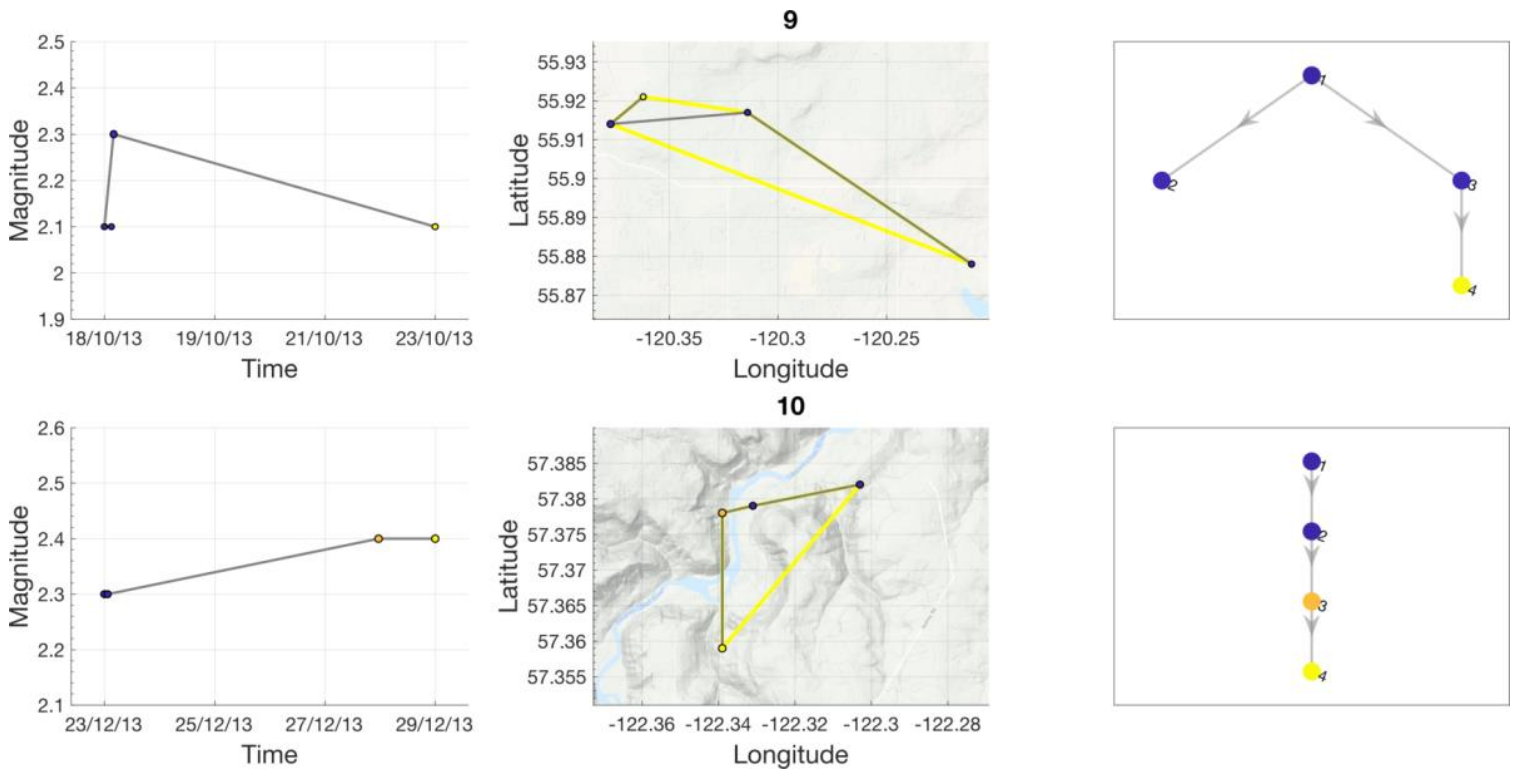

Figure 4-17: Event Family Structures within the Montney cluster 2. a) Moment magnitude vs. Time in days. b) Spatial map. Yellow border outlines the hull area occupied by the sequence. c) Directed tree graph in dimensionless space. Data points are coloured chronologically from darkest to lightest. 
Table 4-11: Parameter sets for event families within the Montney cluster 2. $\mathrm{N}$ is the size of the sequence, $\langle d\rangle$ is the average leaf depth, $\delta$ is the normalized leaf depth, $B_{i}$ is the inverted branching number, $A$ is the spatial area, $t_{d}$ is the decay period, and $\Delta M$ is the magnitude differential.

\begin{tabular}{cccccccccc}
\hline Sequence & $\mathbf{N}$ & $\langle\boldsymbol{d}\rangle$ & $\boldsymbol{\delta}$ & $\boldsymbol{B}_{\boldsymbol{i}}$ & $\boldsymbol{A}\left(\mathbf{k m}^{2}\right)$ & $\boldsymbol{t}_{\boldsymbol{d}}$ (days) & $\boldsymbol{\Delta} \boldsymbol{M}$ & Class \\
\hline $\mathbf{1}$ & 17 & 4.00 & 0.97 & 0.62 & 7.41 & 5.44 & 0.10 & Swarm \\
$\mathbf{2}$ & 16 & 1.64 & 0.41 & 0.33 & 194.01 & 198.48 & 1.20 & Burst \\
$\mathbf{3}$ & 7 & 5.00 & 1.89 & 0.83 & 6.97 & 9.87 & 0.30 & Swarm \\
$\mathbf{4}$ & 7 & 3.00 & 1.13 & 0.67 & 2.13 & 2.51 & 0.10 & Swarm \\
$\mathbf{5}$ & 6 & 3.00 & 1.22 & 0.80 & 5.09 & 4.47 & 0.10 & Swarm \\
$\mathbf{6}$ & 5 & 1.00 & 0.45 & 0.25 & 9.49 & 9.82 & 0.70 & Burst \\
$\mathbf{7}$ & 5 & 2.50 & 1.12 & 0.75 & 22.47 & 2.09 & 0.00 & Swarm \\
$\mathbf{8}$ & 5 & 4 & 1.50 & 0.75 & 0.67 & 10.94 & 5.03 & 0.20 & Swarm \\
\hline
\end{tabular}

The ETAS model application using the estimated $M_{c}=2.5$ is presented in Figure 4-18 and its parameters are summarized in Table 4-12. The model fits well; a QOF of 0.02 indicates that the majority of seismicity is being closely matched by the modeled rate. The $p$ value is low, similar to the previous period, which agrees with the largest burst sequence and contradicts the rest characterized by short decay periods. Once again, this may or may not be due to the addition of unrelated but temporally close events being grouped together by the model. The $\alpha$ parameter is low, indicating a lack of dependence on magnitude in the generation of aftershocks. This is a 
potentially interesting result, as low $\alpha$ values have been shown to correlate with magnitudeinvariant, swarm-like seismicity (Ogata, 1987b; Ogata, 1992), which is what the NND model picks up over this time frame.

a)

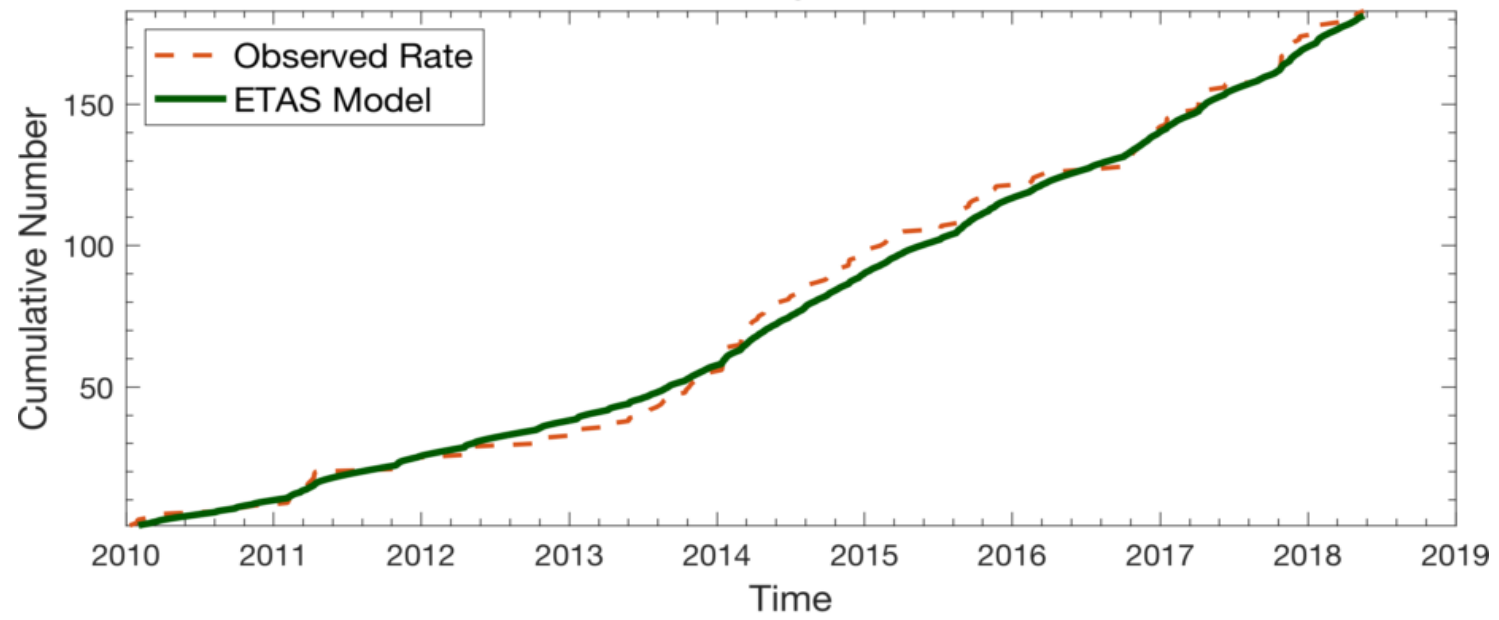

b)
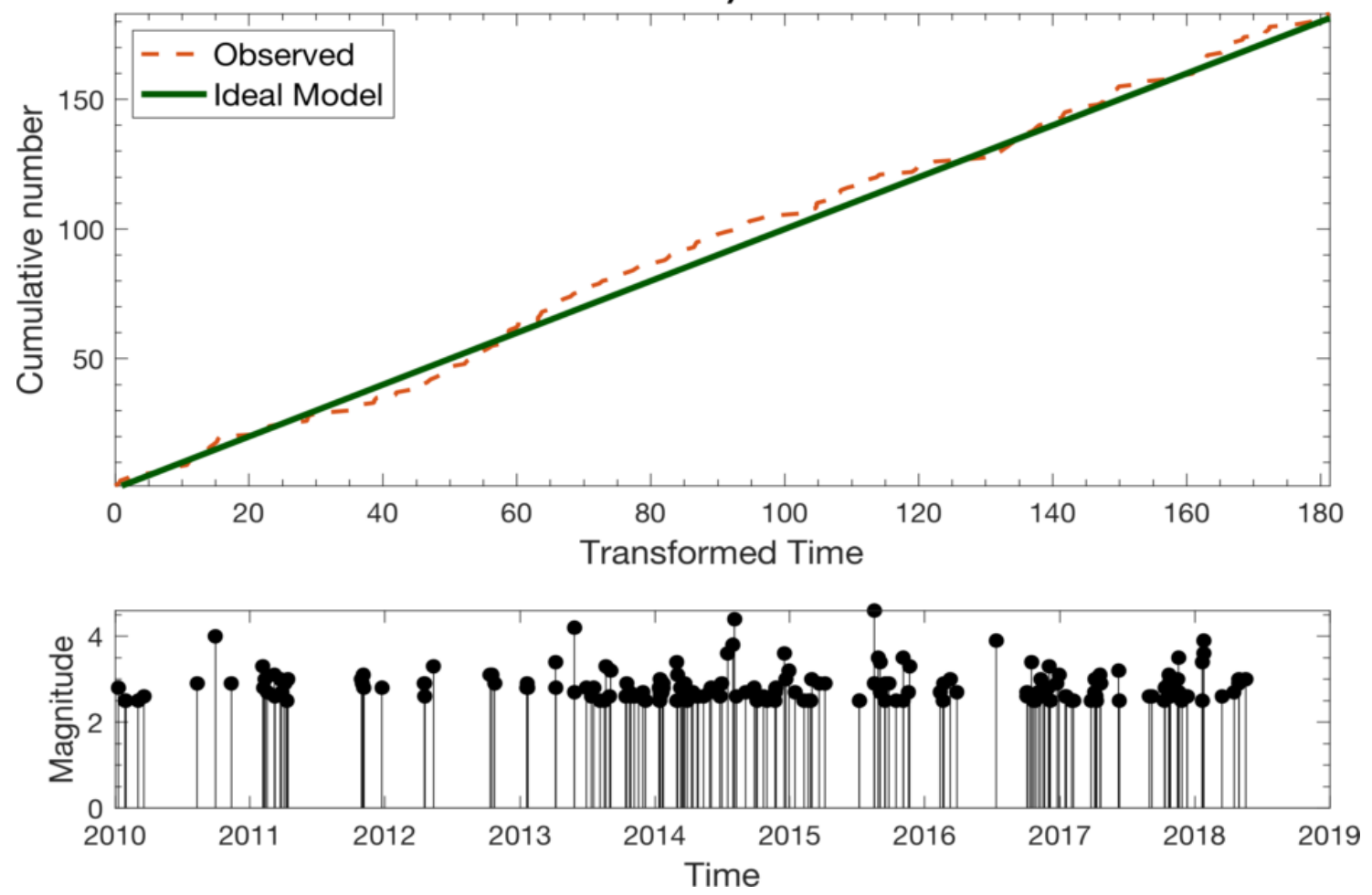

Figure 4-18: ETAS Model of the Montney cluster 2. a) Original time plot. Dashed orange line is the observed cumulative rate of seismicity. Solid line is the optimized model. b) Transformed time plot. Solid line represents the "perfect" model fit. 
Table 4-12: ETAS model parameters for the Montney cluster 2. $\mathrm{N}$ is the number of events modeled, $\mu$ is the constant background rate, $K$ is the aftershock productivity, $c$ is the temporal offset, $p$ is the exponential decay, $\alpha$ is the magnitude-aftershock dependence, and QOF is the quality of the model fit.

\begin{tabular}{lccccccc}
\hline$M_{\boldsymbol{c}}$ & $\mathbf{N}$ & $\boldsymbol{\mu}$ (Events/Day) & $\boldsymbol{K}$ (Events/Day) & $\boldsymbol{c}$ (Days) & $\boldsymbol{p}$ & $\boldsymbol{\alpha}$ & $\mathbf{Q O F}$ \\
\hline 2.5 & 183 & 0.016 & 1.0 & 0.022 & 0.84 & 0.39 & 0.02 \\
\hline
\end{tabular}

\subsection{Fox Creek Cluster (FCC) - Hydraulic Fracturing (2013- 2018)}

Conventional production in central Alberta, primarily within the Duvernay, Swan Hills and Leduc formations, has been occurring since the 1960s and resulted in negligible associated seismicity. However, in December 2013, earthquakes began transpiring approximately 30-40 km west of the town of Fox Creek, where HF wells had recently been drilled in order to access the Duvernay's reservoirs of tight, bituminous shale. Several hundreds of these wells have been drilled since 2012 within the Kaybob South, Waskahigan and McKinley fields near the recent clustering, and a large proportion were drilled horizontally or at a deviated angle in order to engage a greater volume of the hydrocarbon-rich layers. Seismic activity began as a few distinct sequences near Crooked Lake and continues to form further clusters up to the present day (Bao \& Eaton, 2016; Clerc et al., 2016; Deng et al., 2016; Schultz et al., 2016, 2017, 2018; Wang et al., 2016; Eaton et al., 2018; Zhang et al., 2019).

The database for $\mathbf{M} 2+$ events in this area is quite large, which is likely due to the higher resolution of the local network combined with the size and scale of HF operations occurring. The unique geology of the area may also play a role in the manifestation of extended clustering, such as more numerous hydraulic channels and/or closer proximity to well-oriented faults or fossil reef margins, as discussed in Schultz et al. (2016) and Pawley et al. (2018). Regardless, the timing of the abrupt increase in earthquake rate and the large-scale implementation of 
hydraulic fracturing is likely not coincidental, and many of the seismic sequences have been directly attributed to specific well pads (Schultz, 2017). In the analysis of the FCC below, events occurring between December 2013 and September 2018 within a $45 \mathrm{~km}$ radius of the coordinates $[-117.4,54.4]$ were considered (Figure 4-19).

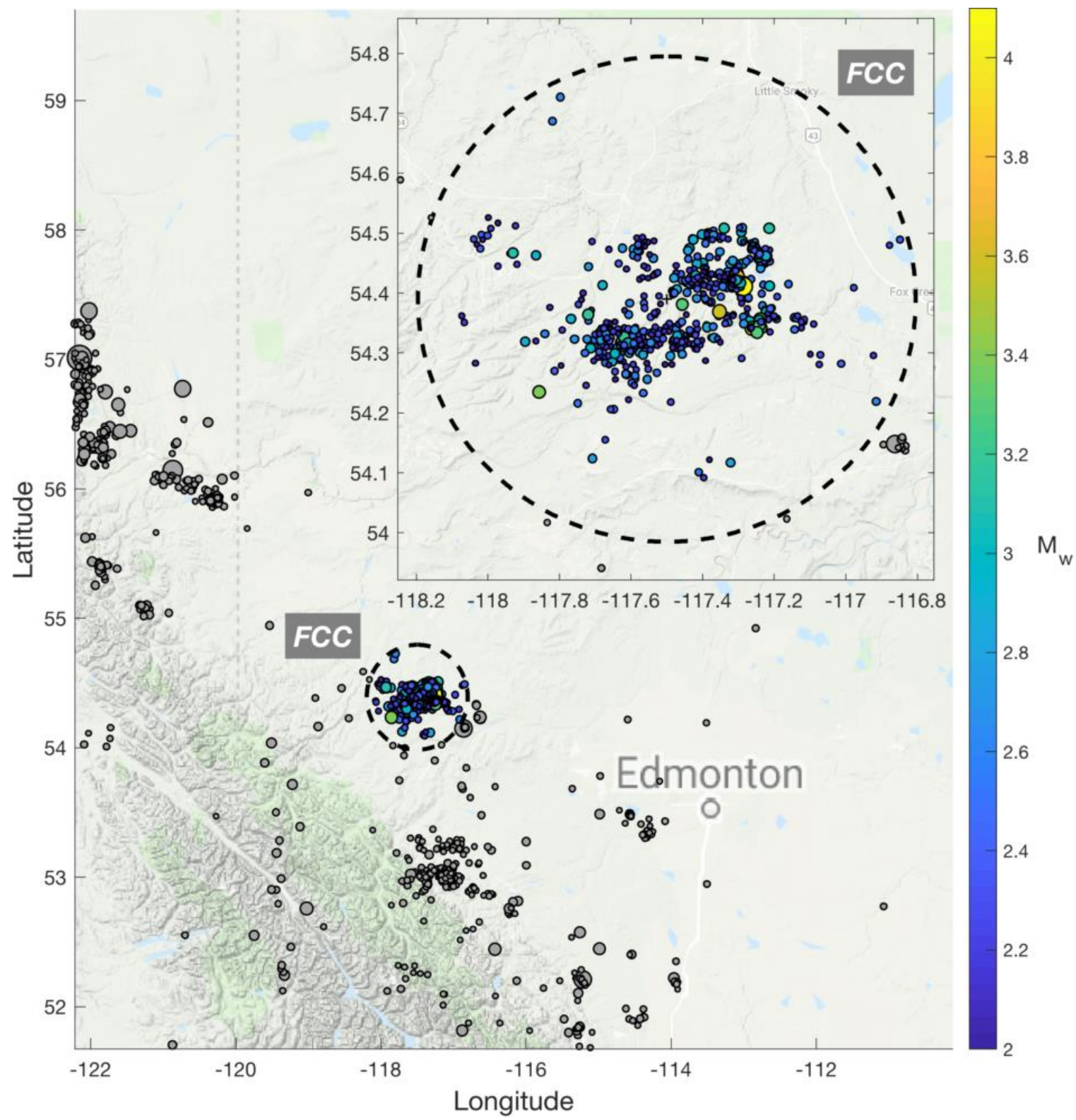

Figure 4-19: Map of the Fox Creek cluster study area between 2013-2018. Dashed circle represents a $45 \mathrm{~km}$ radius from the coordinates [-117.4, 54.4]. Markers are seismic events. Coloured markers are the data points used for analysis. 
The completeness magnitude and Gutenberg-Richter analyses results are displayed in Figure 4-20 and listed in Table 4-13. The FMD is very steep, indicating a preponderance of smaller magnitude events and relatively few larger events. There is only one M4+ event in the dataset. Once again, the FMD peaks early in the distribution and hence the point of maximum curvature (MAXC) is likely an underestimation of $M_{c}(M=2.1$, panel a). The GFT method calculates a best-fitting synthetic distribution at $M=2.5$ (panel b). This concurs with the $b$-value analysis (panel c), which shows that both the $b$-value (blue line) and rolling average $b_{\text {ave }}$ (orange line with triangle markers) stabilize in the region $2.4 \leq M_{0} \leq 2.7$, where confidence bounds are still well constrained.

The GR parameters were computed using $M_{c}=2.5$; the resulting $a$ and $b$-values are high, reflecting both the considerable amount of seismicity occurring over the shorter time frame as well as the large proportion of lower-magnitude events. These results potentially align with findings in other studies, which suggest that phenomena involving significant quantities of migratory or invasive fluids (such as natural processes like subsurface magmatic flow in volcanic regions or dike intrusions near large bodies of water, as well as artificial processes like multistage hydraulic fracturing) tend to trigger seismicity in swarms possibly characterized by higher $b$-values (Rutledge et al., 2004; Morita et al., 2006; Farrell et al., 2009; Vermylen \& Zoback, 2011; Guest et al., 2014; Zaliapin \& Ben-Zion, 2016; Martínez-Garzón et al., 2018). 
a)

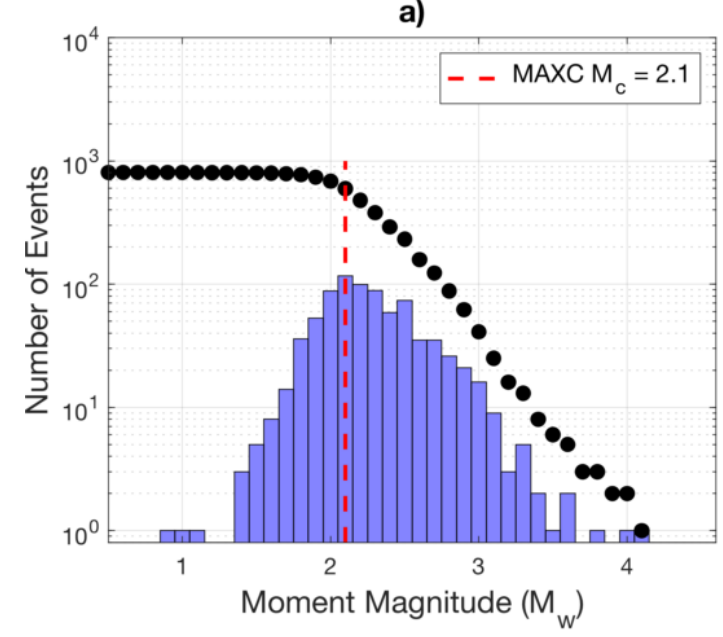

c)

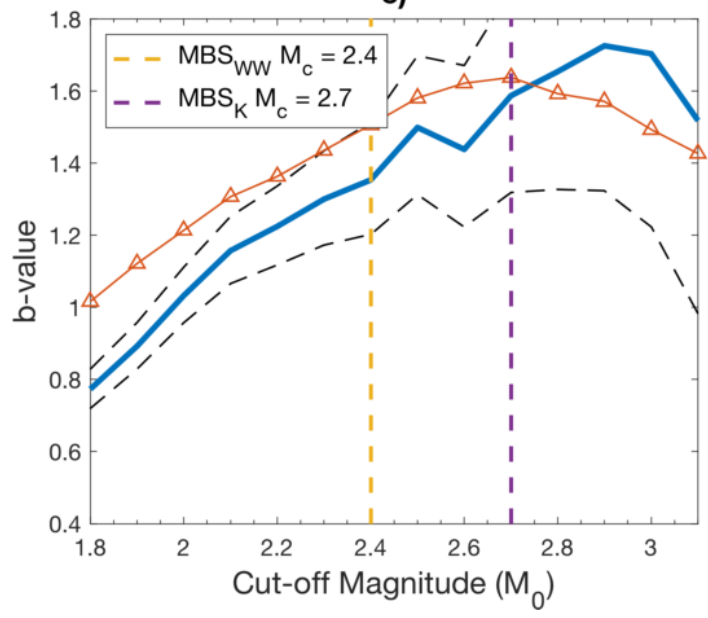

b)

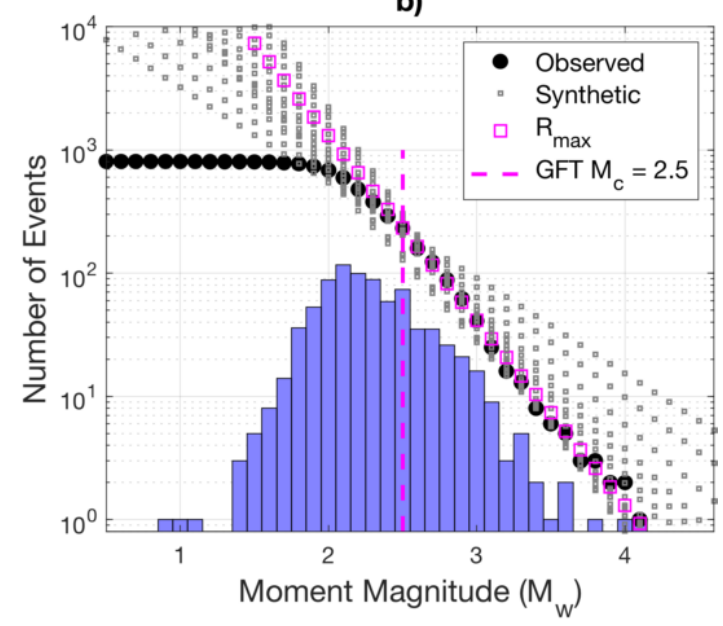

d)

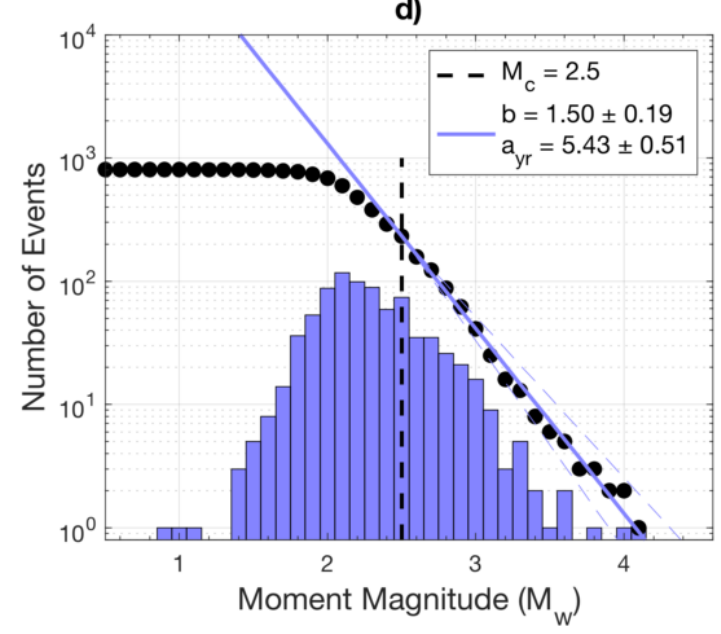

Figure 4-20: $M_{c}$ and GR Analyses for the Fox Creek cluster. a) Maximum Curvature Method (MAXC). b) Goodness-of-Fit Test (GFT). c) Method of $b$-value Stability (MBS). d) GR parameter estimation using determined $M_{c}$.

Table 4-13: Summary of $M_{c}$ and GR Analyses for the Fox Creek cluster.

\section{N MAXC GFT MBSww MBSk Determined $M_{c} \quad b \quad a$}

$\begin{array}{llllllll}806 & 2.1 & 2.5 & 2.4 & 2.7 & 2.5 & 1.50 \pm 0.19 & 5.43 \pm 0.51\end{array}$

The joint distribution of rescaled inter-event time and distance as well as the normalized $\eta$ density is displayed in Figure 4-21. The results contrast strikingly with those obtained for the previous clusters, as there is a single dominant mode located in the tightly clustered domain, 
while only a faint cloud of loosely clustered events is discernable. In a natural setting, the NND distributions would suggest that a given background event occurring in this area has a much higher probability of stimulating further seismicity. However, based on the FCC's spatial and temporal relationship with the surrounding hydraulic fracturing activity, as noted in other studies (e.g. Atkinson et al., 2016; Bao \& Eaton, 2016; Schultz et al., 2017), it appears likely that external factors are contributing directly to the triggering of tightly clustered earthquakes. The FCC's joint distribution also bears a strong resemblance to that of southern California, as defined by Schoenball et al. (2015) surrounding the Coso Geothermal Field (CGF) (Figure 5-2; discussed in Chapter 5). The dominant clustered mode there is attributed mainly to tectonic and magmatic activity; the migratory fluid processes involved in HF operations may stimulate seismicity in the FCC much in the same manner as the flowing magma near the CGF. 
a)

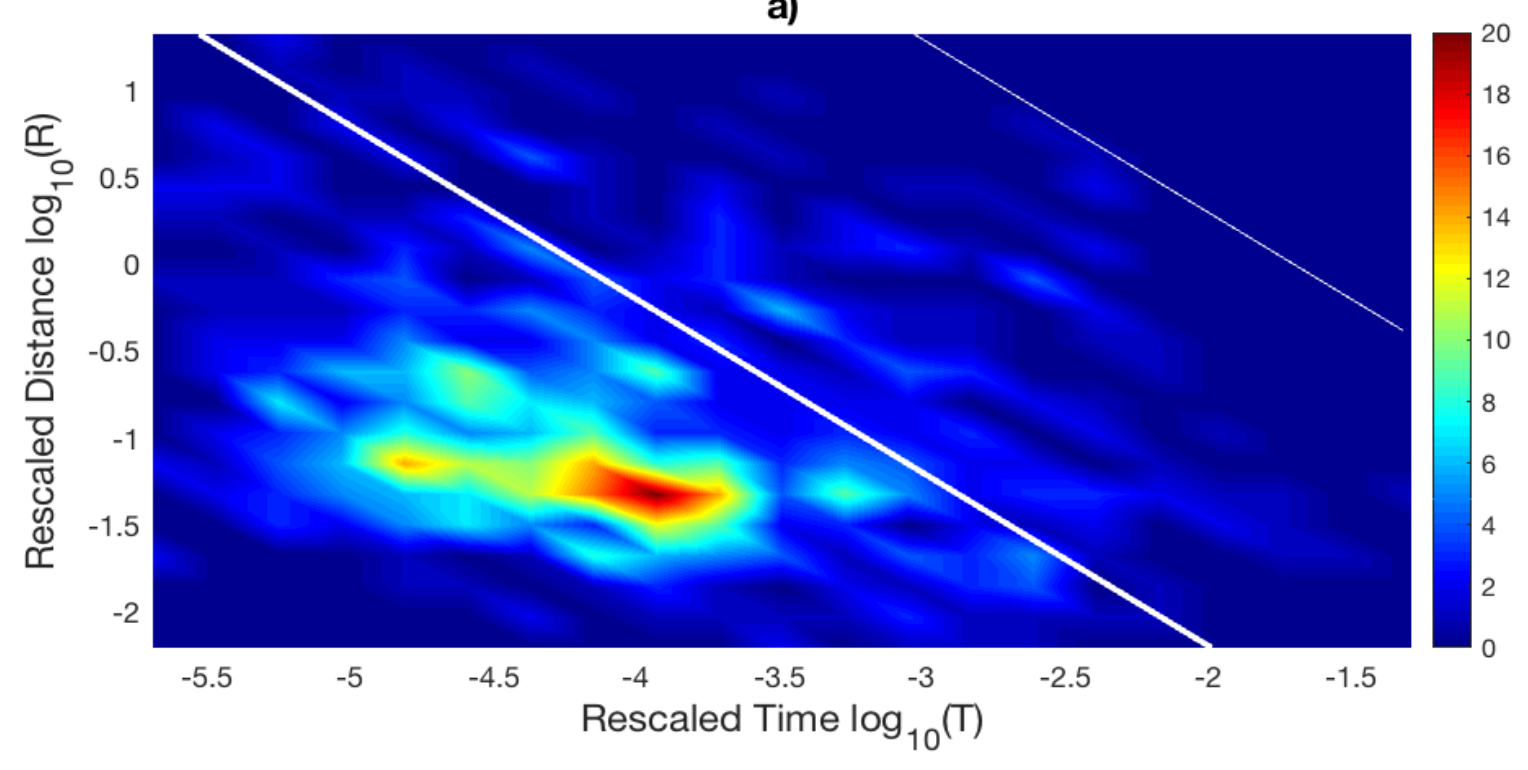

b)

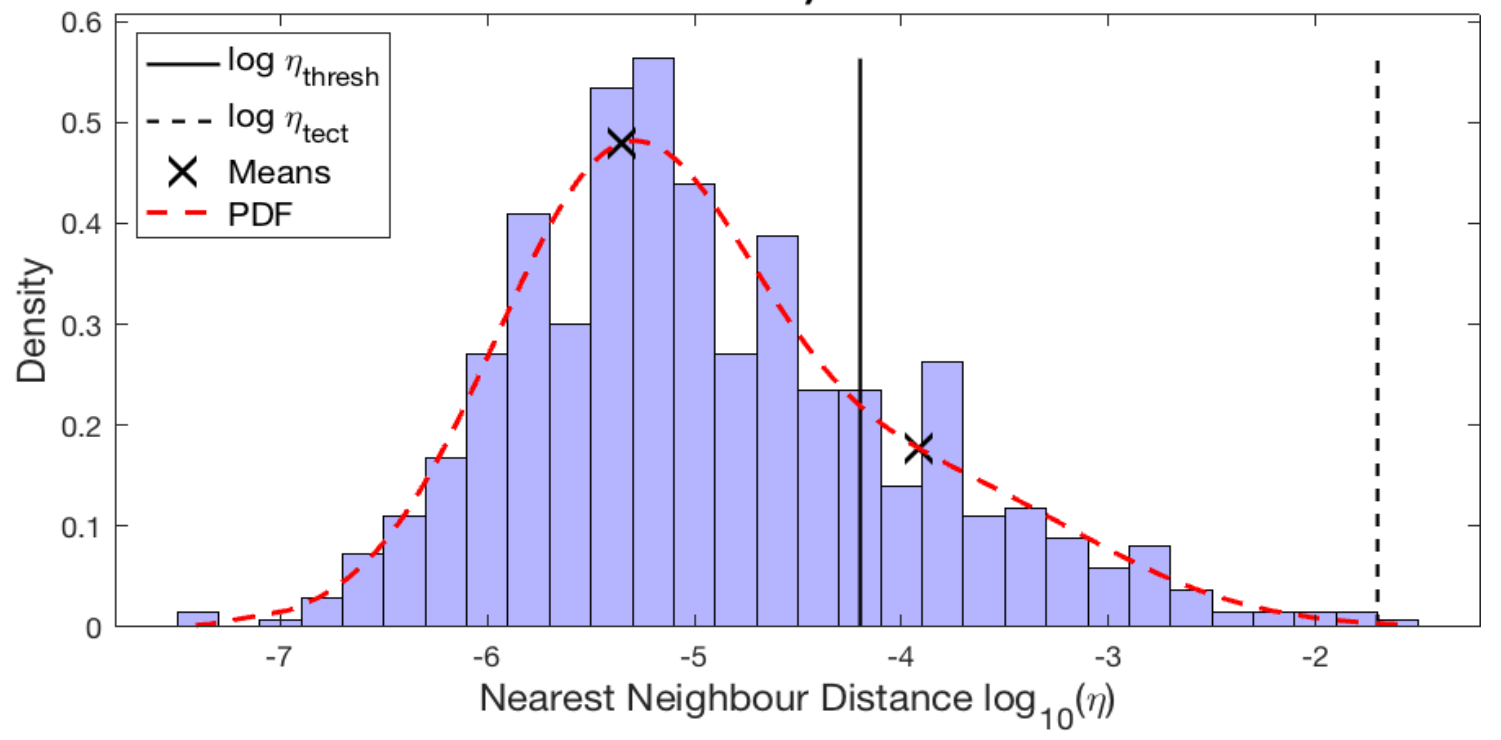

Figure 4-21: Nearest-Neighbour Distance distribution for the Fox Creek cluster. a) Joint distribution of the temporal and spatial components $(T, R)$. b) Normalized density of $\eta$ values.

Table 4-14: Event Family Breakdown for the Fox Creek cluster.

Singles Foreshocks Mainshocks Aftershocks Aftershocks of Aftershocks

$95(14.3 \%) \quad 142(21.4 \%) \quad 41(6.2 \%) \quad 386(58.1 \%) \quad 286(43.1 \%)$


The event-class breakdown of foreshocks, mainshocks, aftershocks, higher order aftershocks and singles is summarized in Table 4-14 and plotted in Figure 4-22. The progressive growth of earthquake magnitude and event-family size is apparent in Figure 4-22. During the first active year, four small-to-moderate clusters are observed, which all contain lower-magnitude mainshocks. From 2015 onward, a series of large, separable clusters containing many foreshocks, aftershocks and higher order aftershocks occur at a rate of approximately 2-3 sequences per year. A large aftershock sequence containing the largest magnitude mainshock is distinguishable in early 2016 . There is a paucity of single events $(95 ; 14 \%)$ and also relatively few mainshocks $(41 ; 6 \%)$, implying that all remaining earthquakes in the dataset $(528 ; 80 \%)$ are strongly linked as part of event families. Over half of these remaining events are identified as higher order offspring $(286 ; 43 \%)$ suggesting that the clusters exhibit elevated levels of interevent triggering, which is a characteristic of swarm sequences. This is in contrast with typical aftershock sequences, where the majority of events connect directly to the mainshock.

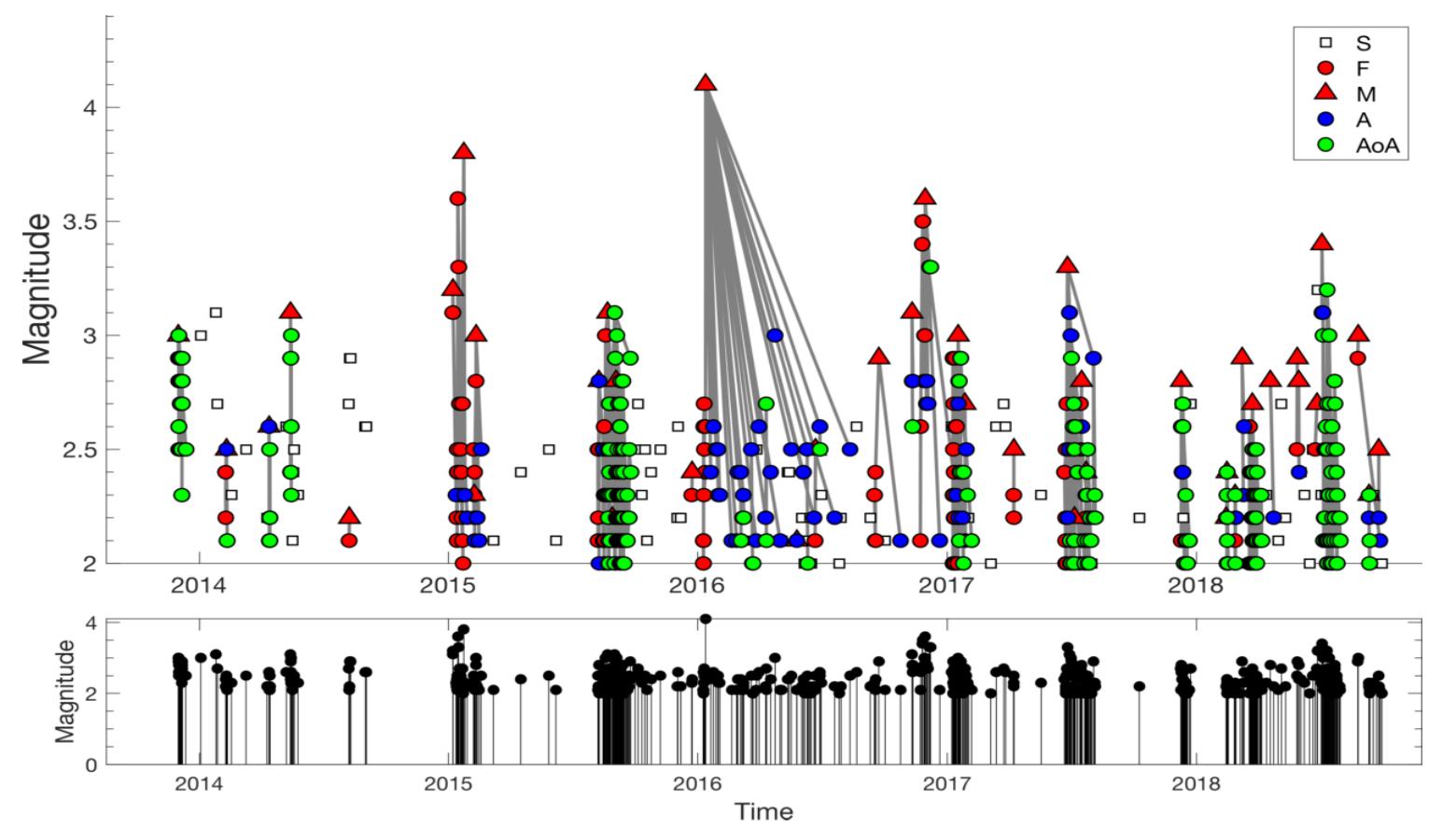

Figure 4-22: Magnitude-Time evolution of event families within the Fox Creek cluster. Lower plot: Event magnitudes vs. time. Upper plot: Event-type classification over time. White squares are singles, red circles are foreshocks, red triangles are mainshocks, blue circles are aftershocks, and green circles are aftershocks-of-aftershocks. Grey lines represent the strong links connecting individual event families. 
The NND algorithm identifies 16 significant event families overall; 8 of which contain 20 or more events, and 4 contain more than 40. These are shown in their structural representations in Figure 4-23 and their parameters are given in Table 4-15. 12 of the 16 families are classified as swarms (including the largest 3), which are chain-like in time with deep family trees containing many higher-order offspring. Topologically, the swarms are characterized by large inverted branching numbers and normalized leaf depths as well as have small magnitude differentials. They cover spatial areas that scale with the size of the sequence, but also appear to decay relatively quickly (typically persist less than one month). By contrast, the sole burst sequence (cluster 4) continues for 7 months and contains many first-generation aftershocks with relatively few higher-order offspring. It is further characterized by a large magnitude differential and low inverted branching number and normalized leaf depth. The burst is preceded by 11 small-magnitude foreshocks, suggesting some precursory triggering behavior prior to the mainshock. The remaining 3 sequences (clusters 5, 6, and 14) are classified as swarm-bursts, which comprise of traits from both sequence types. Each of these clusters begins with several low magnitude foreshocks that lead to a relatively large mainshock, which then causes a spray of aftershocks. As such, the swarm-bursts contain more leaves than the more typical swarms. However, in clusters 5 and 6 , the aftershocks tend to chain together gradually and still form deeper family trees as the sequences decay. Topologically, the swarm-bursts have moderate inverted branching numbers and normalized leaf depths.
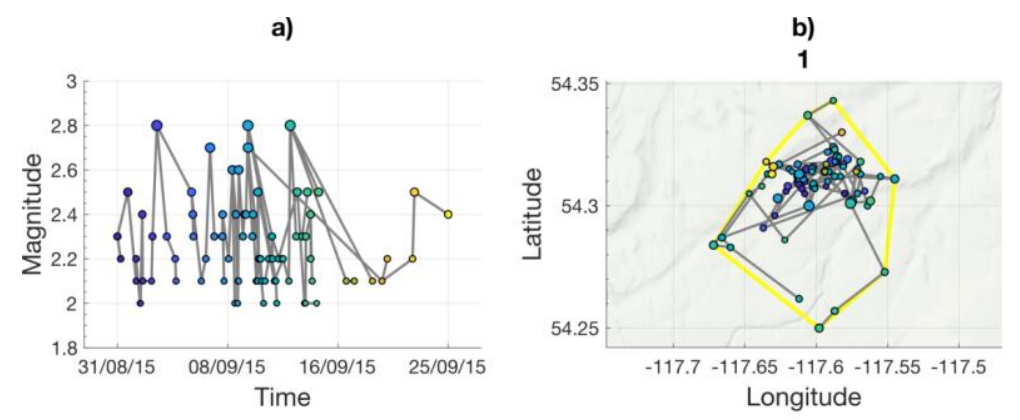

c)
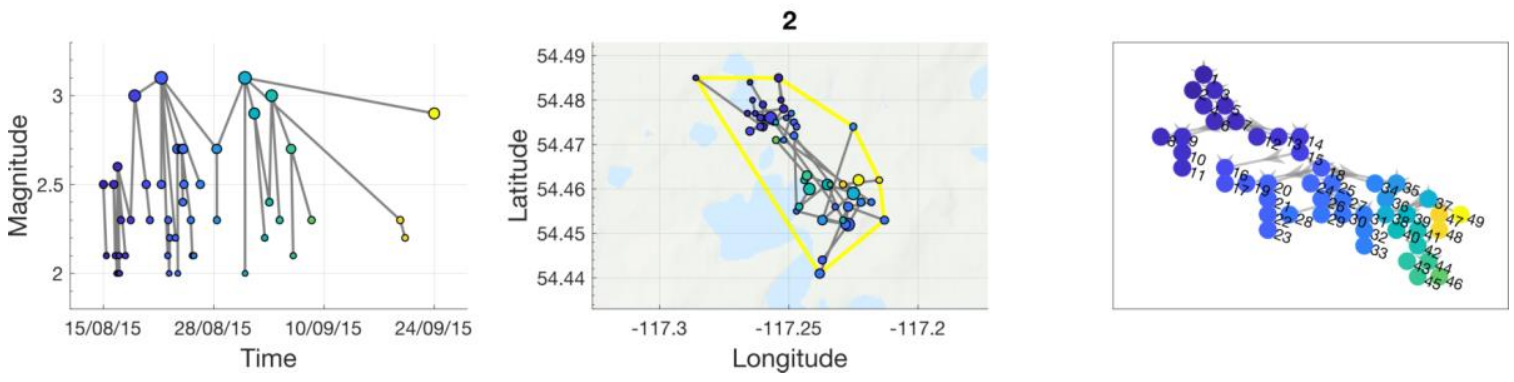

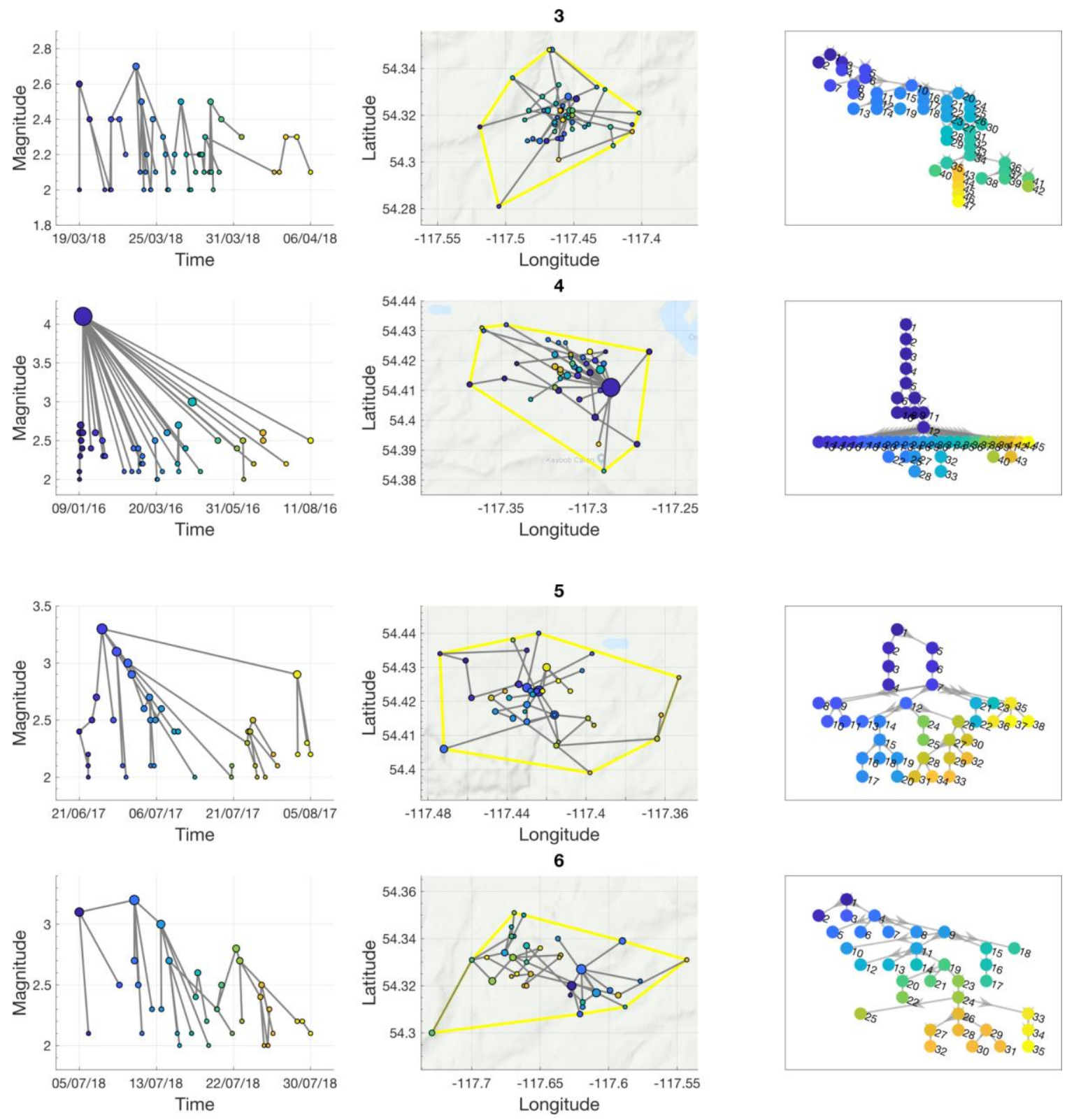

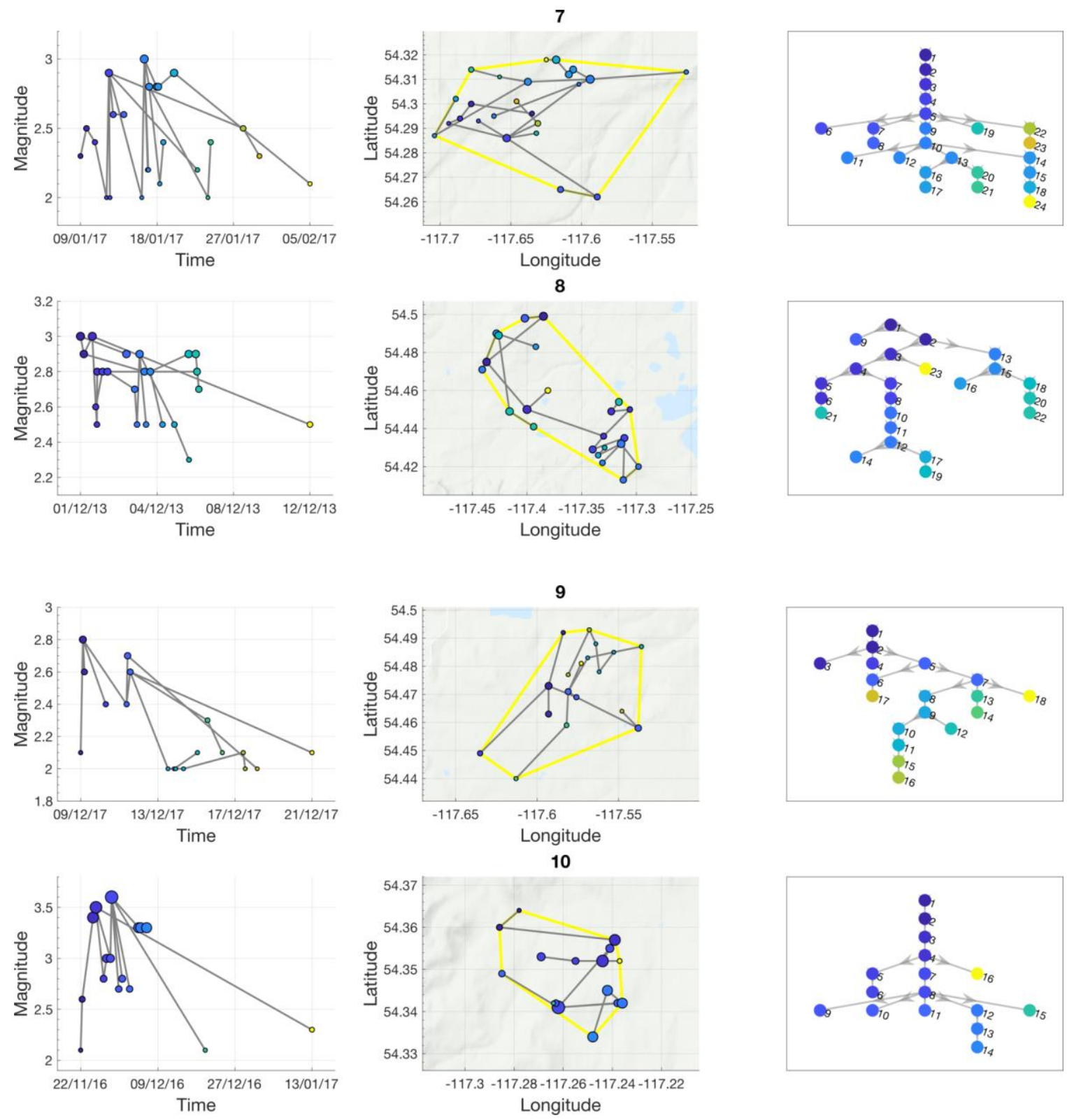

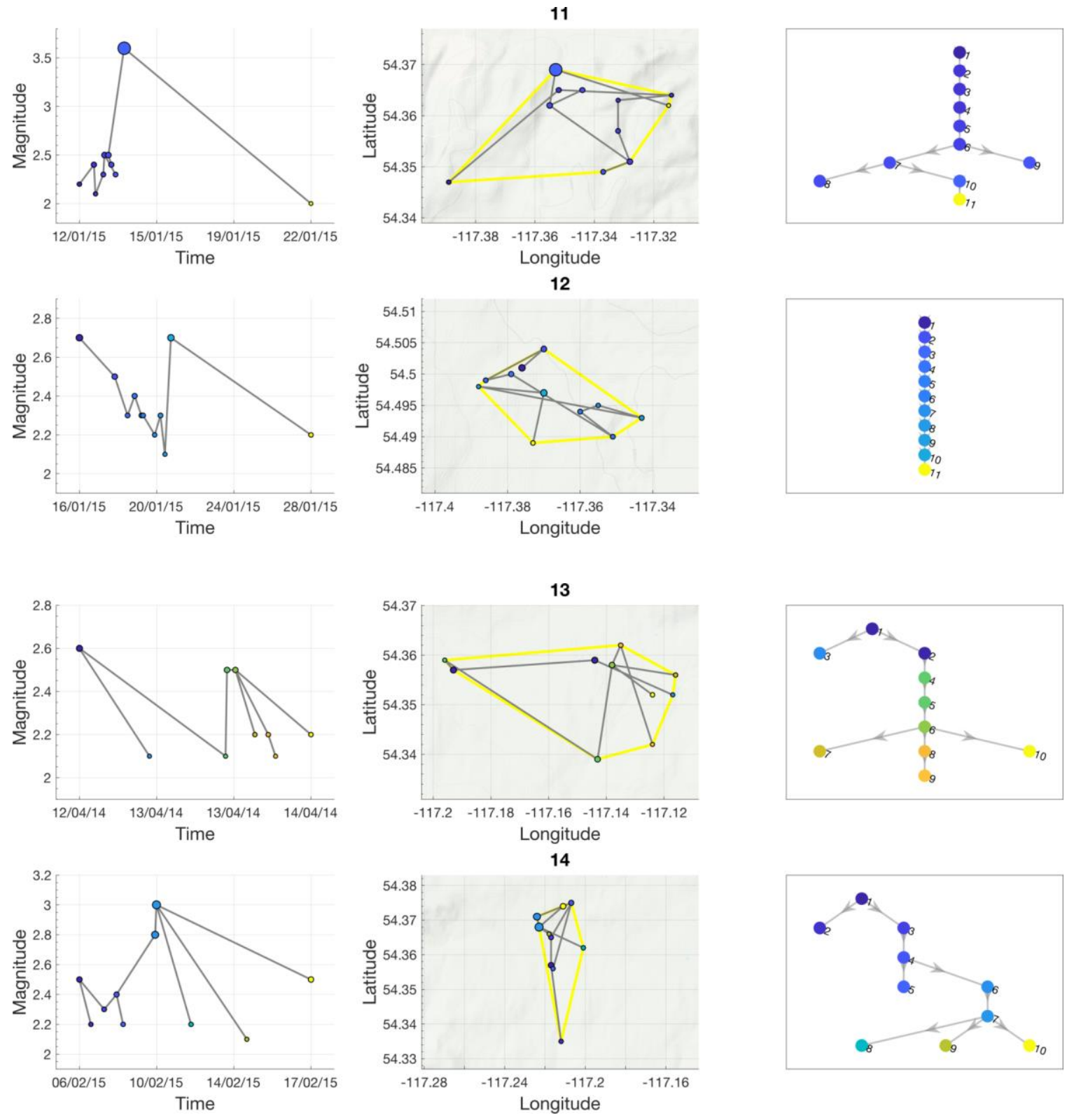

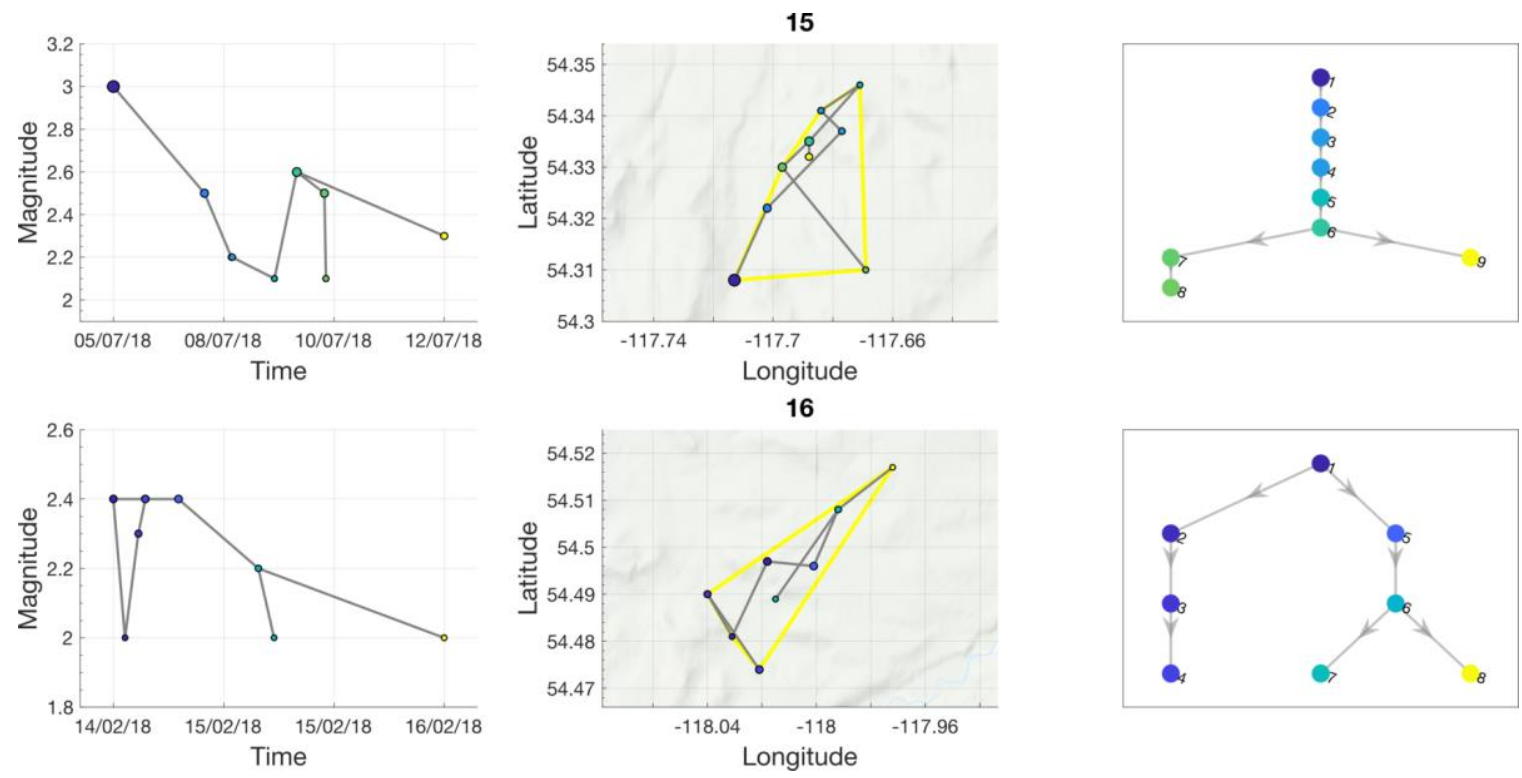

Figure 4-23: Event Family Structures for the Fox Creek cluster. a) Moment magnitude vs. Time in days. b) Spatial map. Yellow border outlines the hull area occupied by the sequence. c) Directed tree graph in dimensionless space. Data points are coloured chronologically from darkest to lightest. 
Table 4-15: Parameter sets for event families within the Fox Creek cluster. $\mathrm{N}$ is the size of the sequence, $\langle d\rangle$ is the average leaf depth, $\delta$ is the normalized leaf depth, $B_{i}$ is the inverted branching number, $A$ is the spatial area, $t_{d}$ is the decay period, and $\Delta M$ is the magnitude differential.

\begin{tabular}{|c|c|c|c|c|c|c|c|c|}
\hline Sequence & $\mathbf{N}$ & $\langle d\rangle$ & $\delta$ & $B_{i}$ & $A\left(\mathrm{~km}^{2}\right)$ & $t_{d}$ (days) & $\Delta M$ & Class \\
\hline 1 & 80 & 28.43 & 3.18 & 0.75 & 50.40 & 24.47 & 0.00 & Swarm \\
\hline 2 & 49 & 7.68 & 1.10 & 0.50 & 11.65 & 40.03 & 0.00 & Swarm \\
\hline 3 & 47 & 9.18 & 1.34 & 0.65 & 31.42 & 18.06 & 0.10 & Swarm \\
\hline 4 & 45 & 8.07 & 1.20 & 0.39 & 63.20 & 215.12 & 1.10 & Burst \\
\hline 5 & 38 & 5.89 & 0.96 & 0.51 & 26.40 & 45.16 & 0.20 & Swarm-Burst \\
\hline 6 & 35 & 4.75 & 0.80 & 0.44 & 37.73 & 25.31 & 0.10 & Swarm-Burst \\
\hline 7 & 24 & 7.11 & 1.45 & 0.65 & 45.35 & 27.38 & 0.10 & Swarm \\
\hline 8 & 23 & 5.57 & 1.16 & 0.73 & 45.97 & 11.01 & 0.00 & Swarm \\
\hline 9 & 18 & 4.57 & 1.08 & 0.65 & 22.68 & 12.05 & 0.10 & Swarm \\
\hline 10 & 16 & 5.86 & 1.46 & 0.60 & 6.96 & 52.53 & 0.10 & Swarm \\
\hline 11 & 11 & 7.00 & 2.11 & 0.80 & 6.76 & 9.41 & 1.10 & Swarm \\
\hline 12 & 11 & 10.00 & 3.02 & 1.00 & 2.71 & 11.16 & 0.00 & Swarm \\
\hline 13 & 10 & 4.25 & 1.34 & 0.67 & 7.82 & 1.40 & 0.00 & Swarm \\
\hline 14 & 10 & 3.80 & 1.20 & 0.56 & 3.48 & 11.11 & 0.20 & Swarm-Burst \\
\hline 15 & 9 & 6.50 & 2.17 & 0.88 & 7.15 & 7.35 & 0.40 & Swarm \\
\hline 16 & 8 & 3.00 & 1.06 & 0.71 & 5.85 & 1.92 & 0.00 & Swarm \\
\hline
\end{tabular}


A cluster map is plotted in Figure 4-24 in order to provide some visual context for the spatial distribution of the detected sequences, which the NND method isolates efficiently. The initial clusters in 2013 and 2014 near Crooked Lake (clusters 8 and 13) are apparent in light purple and green. The more recent sequences in that area (clusters 2, 4, and 5), including the burst sequence, are shown in red, black and magenta. A couple of the largest sequences occur away from the Crooked Lake area to the south end of the FCC (clusters 1 and 3). These detected sequences match quite well with the largest of those identified in Schultz et al. (2015c) and Schultz et al. (2017), who analyzed seismic waveforms and performed double-difference hypocenter relocations to assess the spatiotemporal clustering near Crooked Lake.

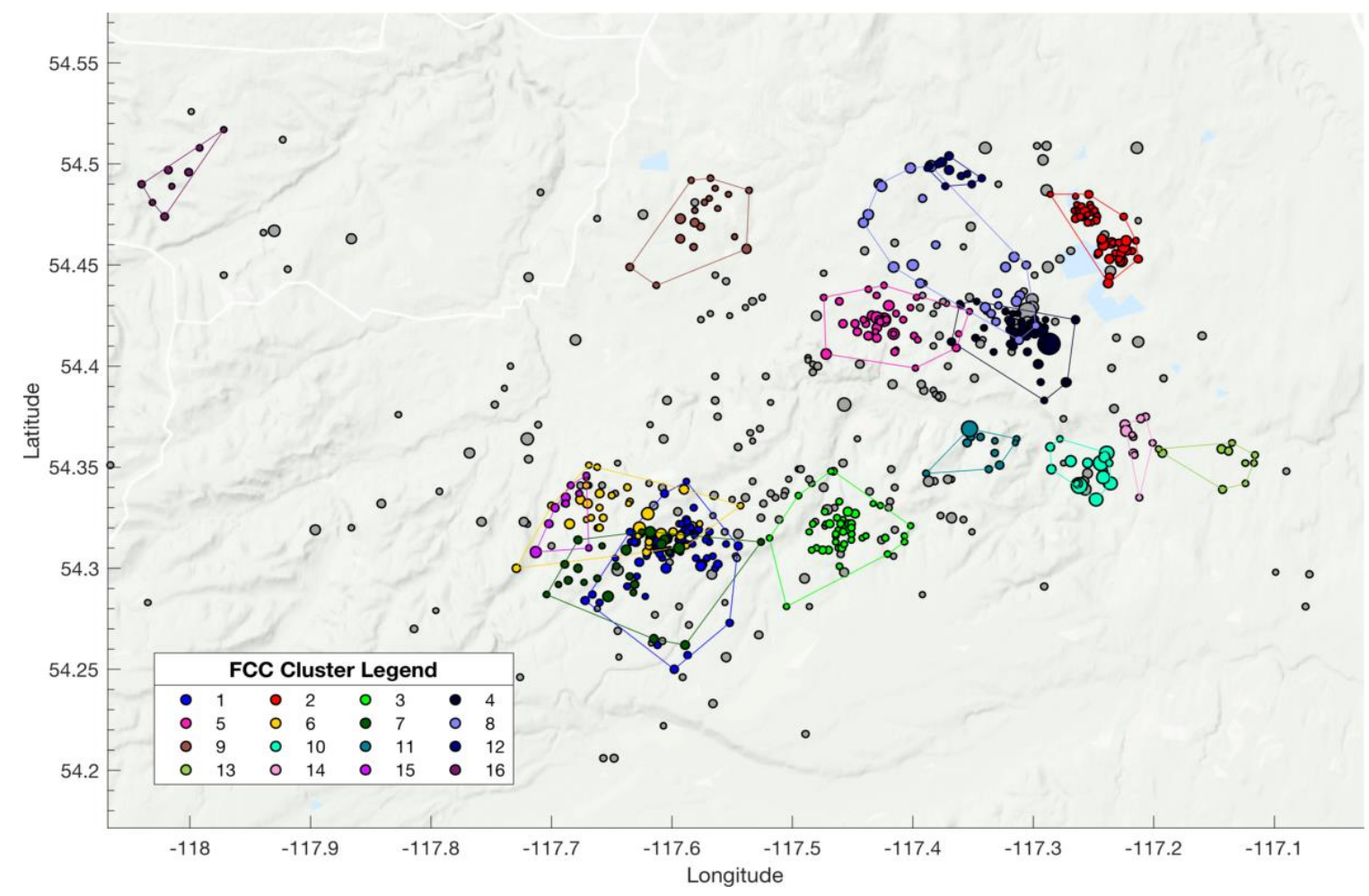

Figure 4-24: Event Sequence Map for the Fox Creek cluster. Coloured markers represent event-family members. Coloured shapes mark the spatial boundaries of each family.

The ETAS model was applied to the FCC using events greater than or equal to the determined completeness magnitude of $M_{c}=2.5$ (Figure 4-25). 8-9 sharp spikes are captured by the modeled rate overall; these correspond to some of the largest isolated sequences identified above. The model fits well visually (plots in transformed time match closely but the model slightly underestimates seismicity throughout, panel b), with a quality-of-fit (QOF) of 0.025, 
but many of the parameters did not converge to realistic values. The analysis was repeated across a range of cut-off magnitudes, which resulted in similar, impossible parameter convergences (Table 4-16).

a)

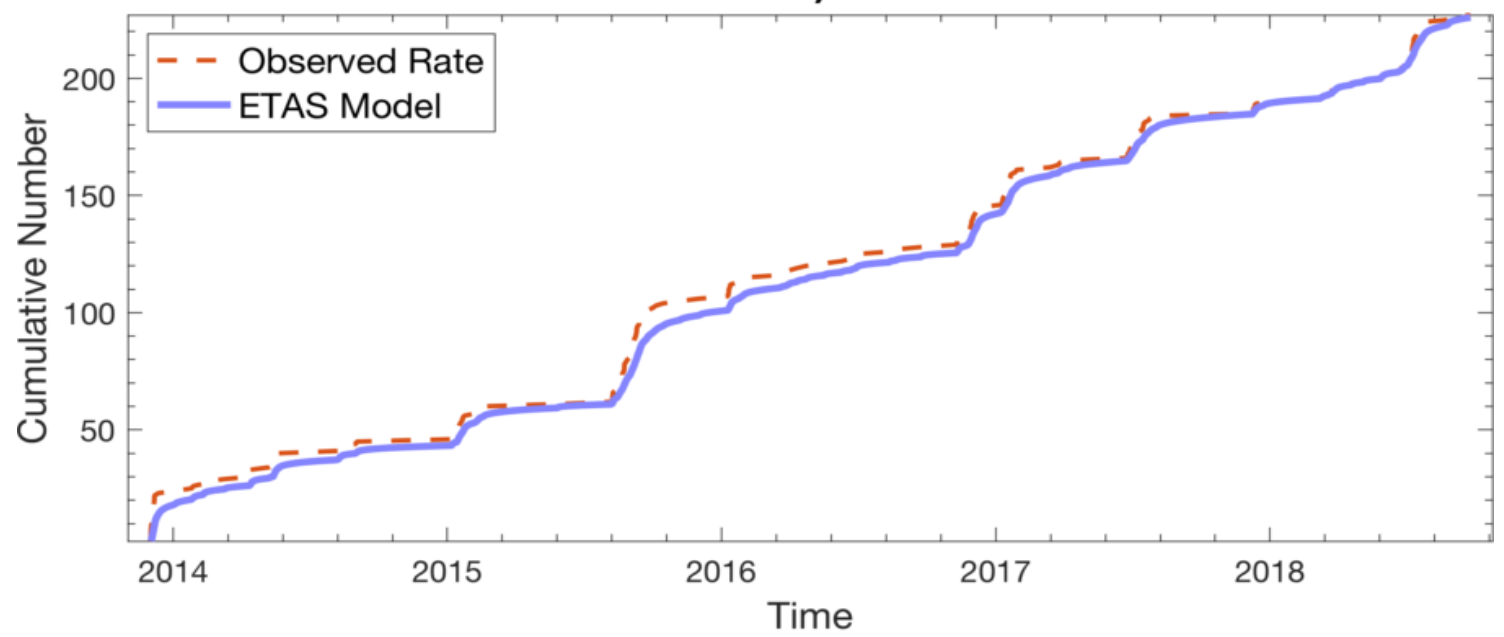

b)
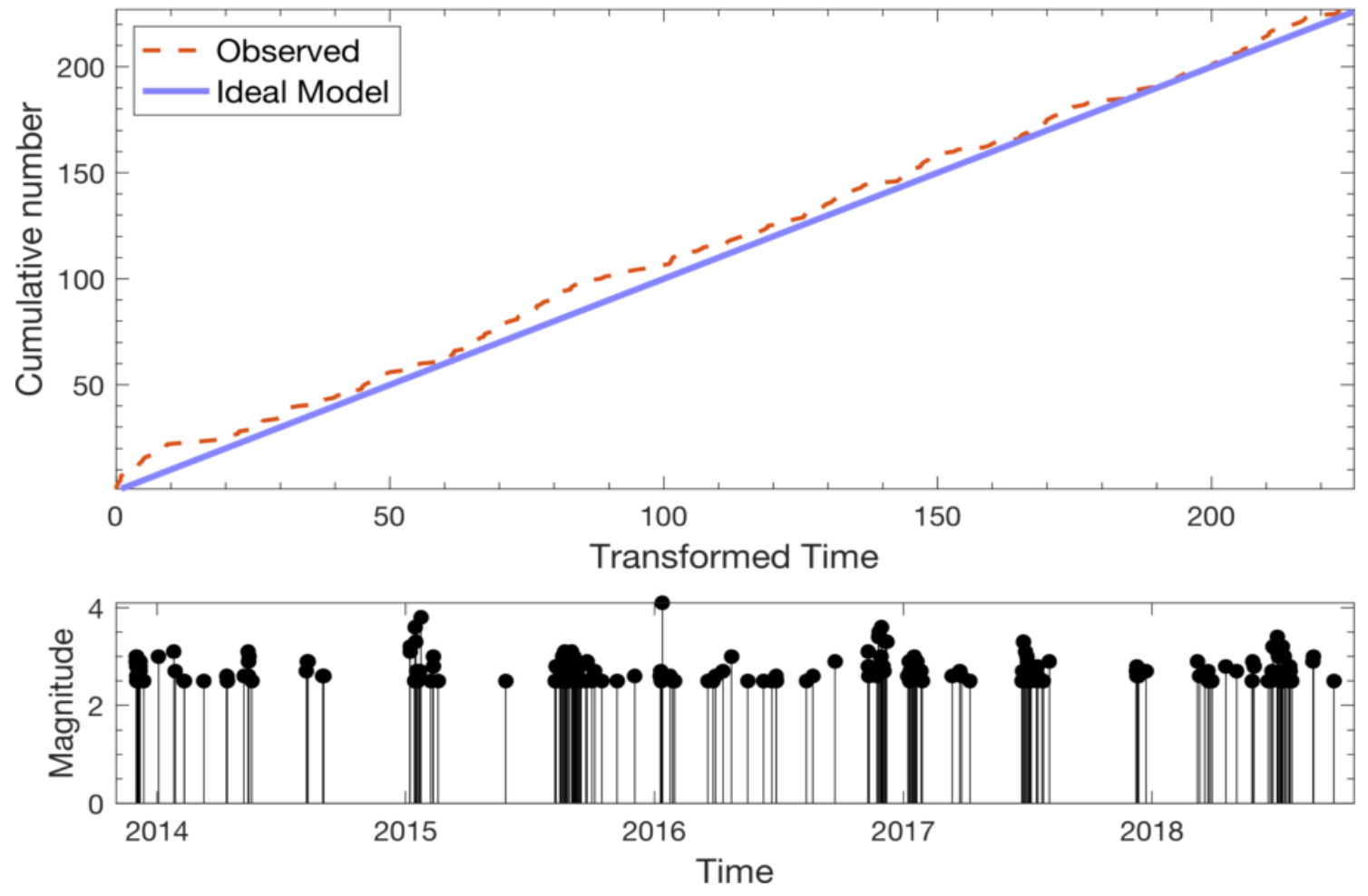

Figure 4-25: ETAS Model of the Fox Creek cluster using $M_{c}=2$. 5. a) Original time plot.

Dashed orange line is the observed cumulative rate of seismicity. Solid line is the optimized model. b) Transformed time plot. Solid line represents the "perfect" model fit. 
Table 4-16: ETAS model parameters for the Fox Creek cluster. $N$ is the number of events modeled, $\mu$ is the constant background rate, $K$ is the aftershock productivity, $c$ is the temporal offset, $p$ is the exponential decay, $\alpha$ is the magnitude-aftershock dependence, and QOF is the quality of the model fit.

\begin{tabular}{lcccccccc}
\hline$M_{\boldsymbol{c}}$ & $\mathbf{N}$ & $\boldsymbol{\mu}$ (Events/Day) & $\boldsymbol{K}$ (Events/Day) & $\boldsymbol{c}$ (Days) & $\boldsymbol{p}$ & $\boldsymbol{\alpha}$ & $\mathbf{Q O F}$ \\
\hline $\mathbf{2 . 5}$ & 227 & 0.038 & 0.29 & 9.23 & 5.13 & 0.33 & 0.025 \\
2.3 & 366 & 0.047 & 0.47 & 3.29 & 3.01 & 0.07 & 0.023 \\
2.4 & 285 & 0.045 & 0.33 & 9.36 & 5.53 & 0.28 & 0.024 \\
2.6 & 154 & 0.028 & 0.29 & 9.98 & 5.56 & 0.38 & 0.029 \\
2.7 & 121 & 0.021 & 0.34 & 4.73 & 3.42 & 0.30 & 0.03 \\
\hline
\end{tabular}

An alternate approach was therefore used to fit the ETAS model, based on the results of the NND analysis. The ETAS $\mu$ parameter is optimized to represent background seismicity as a non-zero constant rate, which allows for the generation of the conditional (seismic history dependent) aftershock rate. Consequently, the $\mu$ parameter may relate to the NND "loosely clustered" subpopulation, in that they both aim to represent the subset of background earthquakes surrounding tightly clustered activity. While the other investigated clusters (RMHC, MC1 and MC2) contained high amounts of loosely clustered activity (Figure 4-3, Figure 4-9, and Figure 4-15), possibly resulting from long-term stress perturbations caused by fluid extraction and/or injection, the FCC is comprised of tightly clustered seismic sequences and very few background events (Figure 4-21), suggesting that external factors may be contributing directly to the triggering of seismicity. The absence of the loosely clustered population was used as motivation for the following adjustment to the ETAS model: the background rate parameter was constrained so that $\mu \approx 0$ and the simulation was re-run, again using $M_{c}=2.5$. An absolute zero background rate would result in the erroneous outcome that zero events occurred overall, so $\mu$ was reduced to a very small positive number. The resulting adjusted ETAS model fit is shown in Figure 4-26, with an improved QOF of 0.012. Table 4-17 gives the new parameter values, which converge to realistic values. 
a)

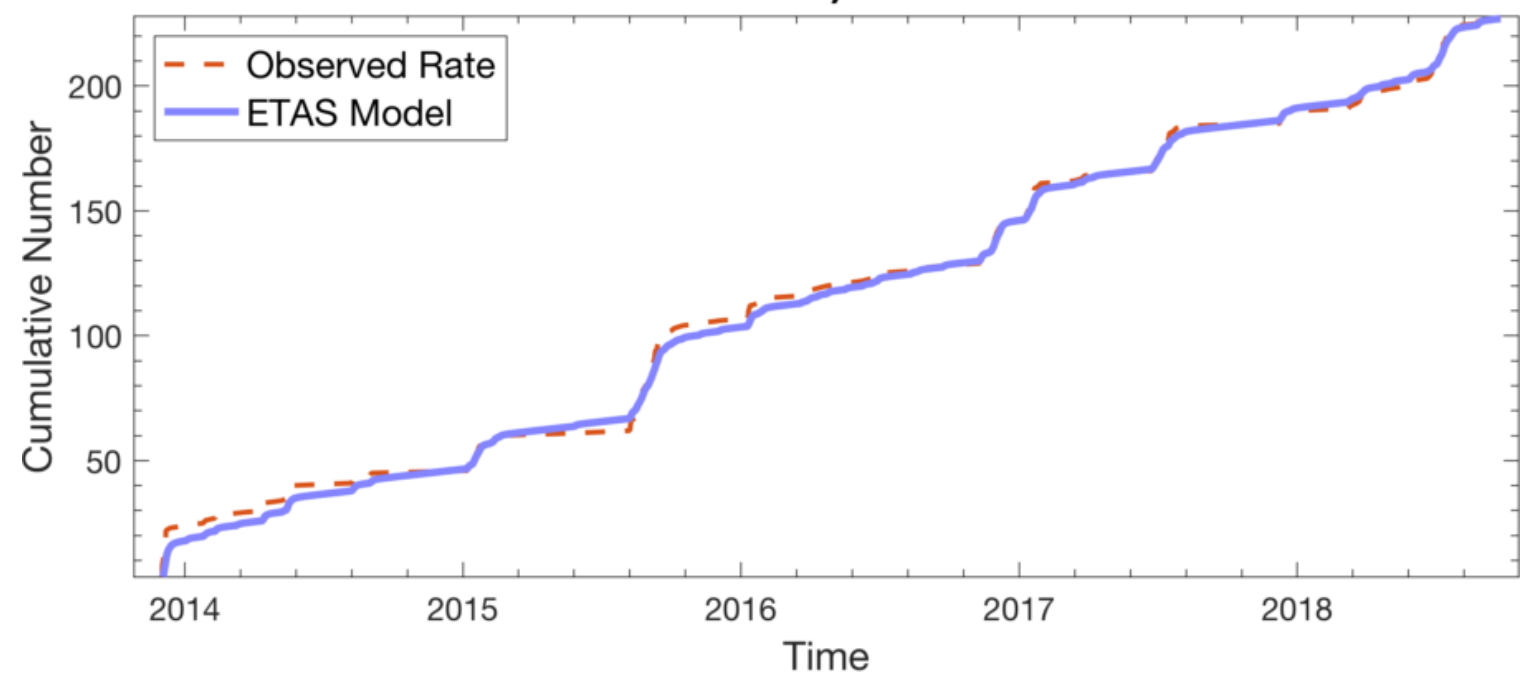

b)
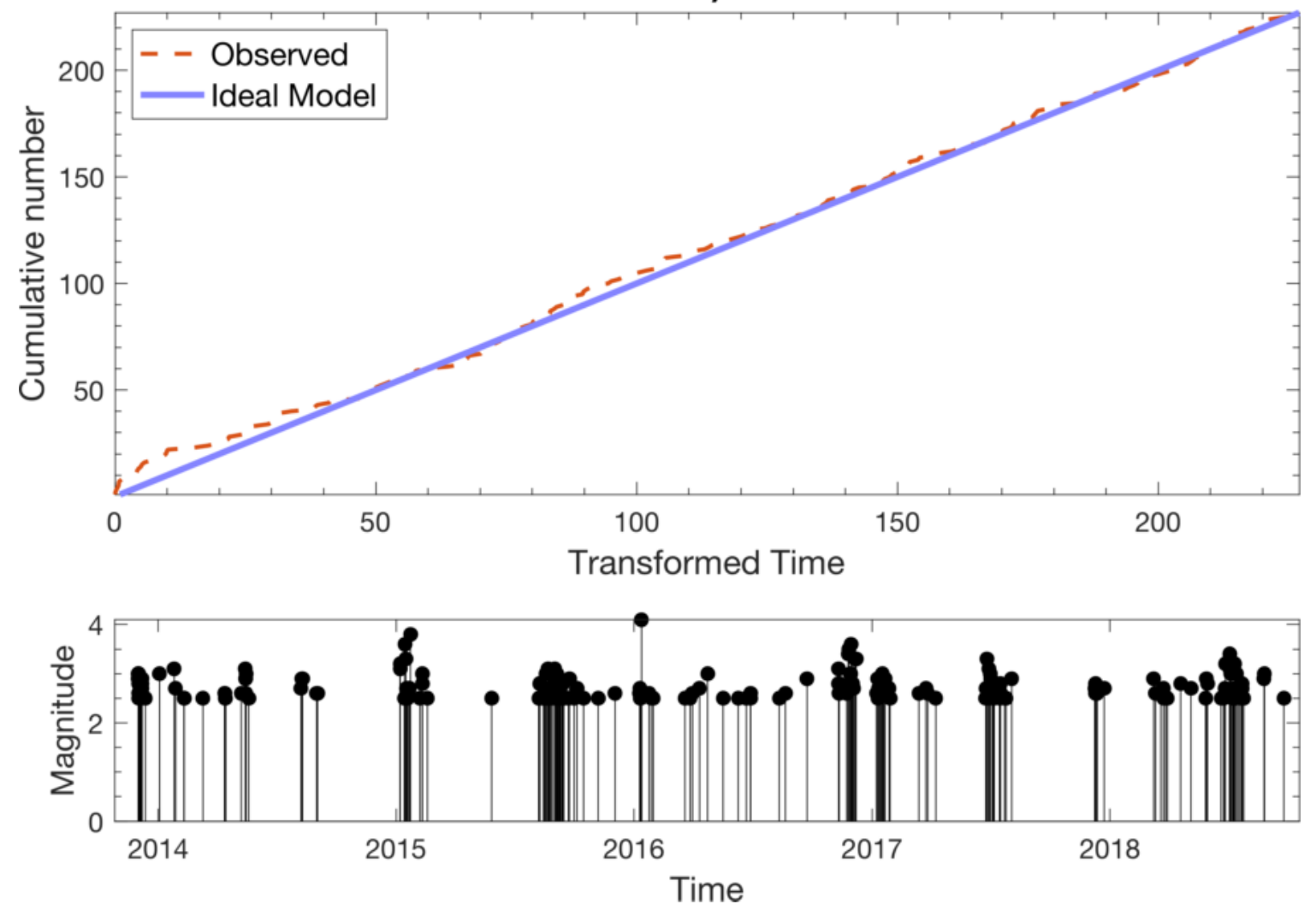

Figure 4-26: ETAS Model of the Fox Creek cluster using $M_{c}=2.5$ and $\mu \approx 0$. a) Original time plot. Dashed orange line is the observed cumulative rate of seismicity. Solid line is the optimized model. b) Transformed time plot. Solid line represents the "perfect" model fit. 
Table 4-17: ETAS model parameters for the Fox Creek cluster using $\mu \approx 0$.

\begin{tabular}{cccccccc}
\hline$M_{\boldsymbol{c}}$ & $\mathbf{N}$ & $\boldsymbol{\mu}$ (Events/Day) & $\boldsymbol{K}$ (Events/Day) & $\boldsymbol{c}$ (Days) & $\boldsymbol{p}$ & $\boldsymbol{\alpha}$ & $\mathbf{Q O F}$ \\
\hline $\mathbf{2 . 5}$ & 227 & 0.00006 & 0.50 & 0.58 & 1.27 & 0.38 & 0.012 \\
\hline
\end{tabular}

The adjusted ETAS model correctly captures approximately a dozen distinct rate spikes, compared to the 8-9 observable sequences in Figure 4-25. The decay parameter $p$ is on the high end of typically observed values, which may demonstrate that the model is accurately identifying the separate clustering sequences, as opposed to grouping unrelated events together. Similar to the recent period of Montney clusters (MC2), the magnitude-weighting parameter $\alpha$ is small, which correlates with the swarm-like behavior observed within most of the detected event families.

\subsection{Discussion}

The case studies above aimed to characterize several of the induced seismic clusters occurring within the WCSB using statistical models. Each model was applied to help illustrate particular features of the seismicity, specifically the frequency-magnitude statistics using the GutenbergRichter (GR) relation, the inter-event distance distributions as well as the nature and extent of closely linked event sequences using the Nearest-Neighbour Distance (NND) method and Epidemic Type Aftershock Sequence (ETAS) model. The results indicate that the investigated clusters are quite unique, differing noticeably in several of the measured statistics while also sharing some similarities in others. The distinctive properties of these clusters, along with their respective similarities and differences, may provide some insight into the potential triggering processes stimulated by their causal mechanisms. A comparative analysis for each model application is provided below.

The frequency-magnitude distributions (FMDs) and estimated GR parameters for each cluster are shown in Figure 4-27. The Rocky Mountain House cluster (RMHC), which was induced by conventional gas extraction, is characterized by a very broad distribution of event magnitudes resulting in a very low $b$-value (panel a, $b=0.74$ ). This contrasts with the Fox Creek cluster (FCC), induced by hydraulic fracturing, whose FMD is very steep and contains many more small magnitude events and fewer large magnitude events. As a consequence, it is described by 
a very high $b$-value (panel d, $b=1.50$ ). Both the wastewater disposal and hydraulic fracturinginduced Montney clusters (MC1 and MC2) have more typical magnitude distributions, with the MC2 modeled by a slightly higher $b$-value (panel b, $b=1.04$ and panel c, $b=1.10$ ). The normalized $a$-values reflect the average yearly-detected seismicity rate. It is not surprising that the more recent, densely populated clusters (MC2 and FCC) have the highest yearly $a$-values, whereas the RMHC and MC1 both span longer periods of time and are smaller overall, resulting in lower yearly $a$-values.
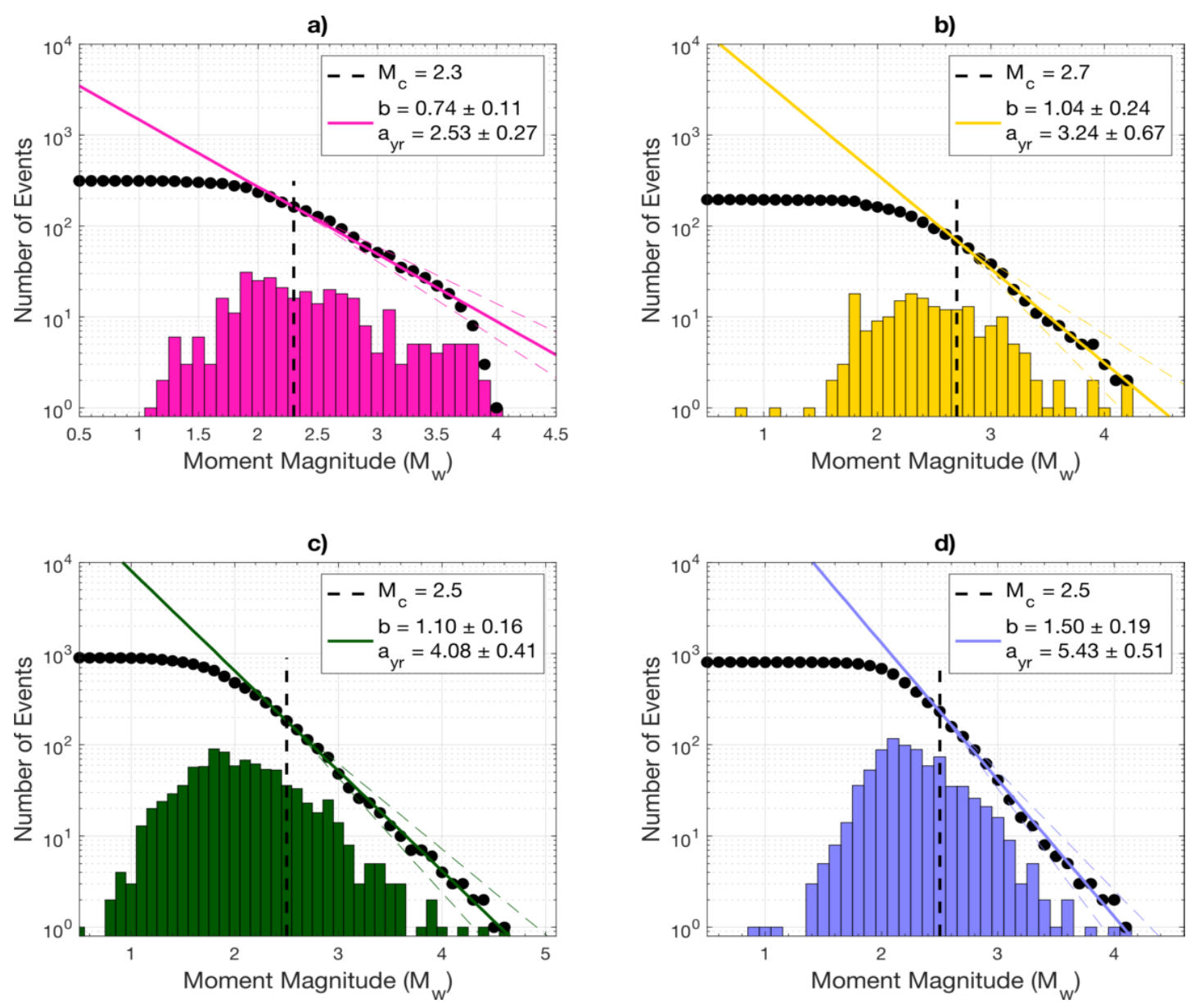

Figure 4-27: Frequency-Magnitude Distributions and estimated GR parameters for each investigated cluster. Coloured histograms represent the non-cumulative magnitude distributions and black dots represent the cumulative magnitude distributions. Dashed black lines indicate the determined local magnitude of completeness. Coloured lines represent the modeled GR relations. a) Rocky Mountain House cluster. b) Montney cluster 1. c) Montney cluster 2. d) Fox Creek cluster. 
The joint space-time NND distributions for each regional cluster, which reflect the mixing proportions of loosely clustered and tightly clustered earthquakes, are plotted in Figure 4-28. As an initial observation, the modal locations of all four clusters provide further confirmation of some of the regional interpretations formed in Chapter 3. Particularly, the results in Chapter 3 suggested that the regional clustering observed within the WCSB occurred within a distinct subset of the multidimensional inter-event NND space, namely within the $\log _{10} \eta \leq \log _{10} \eta_{\text {tect }}$ realm (i.e. within the loosely and tightly clustered domains). The remaining earthquakes were evenly distributed, more closely resembled a Poisson process and occurred largely within the $\log _{10} \eta>\log _{10} \eta_{\text {tect }}$ realm (i.e. within the deep-background domain). From Figure 4-28, it is clear that all of the investigated clusters' inter-event distance distributions do indeed occupy the $\log _{10} \eta \leq \log _{10} \eta_{\text {tect }}$ realm.

However, within the $\log _{10} \eta \leq \log _{10} \eta_{\text {tect }}$ realm, several distinctions between the clusters regarding their mixing proportions are clear. The RMHC (panel a) and MC1 (panel b) distributions are visually similar, containing dominant proportions of loosely clustered events (mostly "singles"). These events transpire somewhat closely together in space and time, but mainly occur as separate instances that rarely trigger further significant seismicity. This loosely clustered activity of single events may reflect the type of seismicity expected to occur within the WCSB due to steady, long-term alterations to the subsurface stress field, which is what was suspected to have eventually triggered both clusters (long-term gas extraction and wastewater disposal, respectively) (Wetmiller, 1986; Horner et al., 1994; Baranova et al., 1999; B.C. Oil and Gas Commission, 2014). Conversely, both the MC2 (panel c) and FCC (panel d) distributions contain distinct modes within the tightly clustered domain. The existence of these modes indicates that a significant proportion of events are occurring very closely together and possibly transpiring as a direct consequence of a triggering mechanism (previous seismicity and/or external forces). The FCC is further distinguishable from the other clusters by its lack of a dominant loosely clustered subpopulation. This is quite significant, as its NND distribution implies either that the small population of background events occurring in this area is capable of triggering massive amounts of tightly clustered activity (despite the fact that other clusters contain higher amounts of loosely clustered seismicity and correspondingly less tight clustering) $o r$ that other, external triggering factors are contributing to the unnatural levels of 
event sequencing. Based on the spatiotemporal correlations made in other studies between the increasing rates of seismicity and the rise in horizontally drilled HF operations, it appears logical to connect HF as one of the probable causative mechanisms (discussed further below).
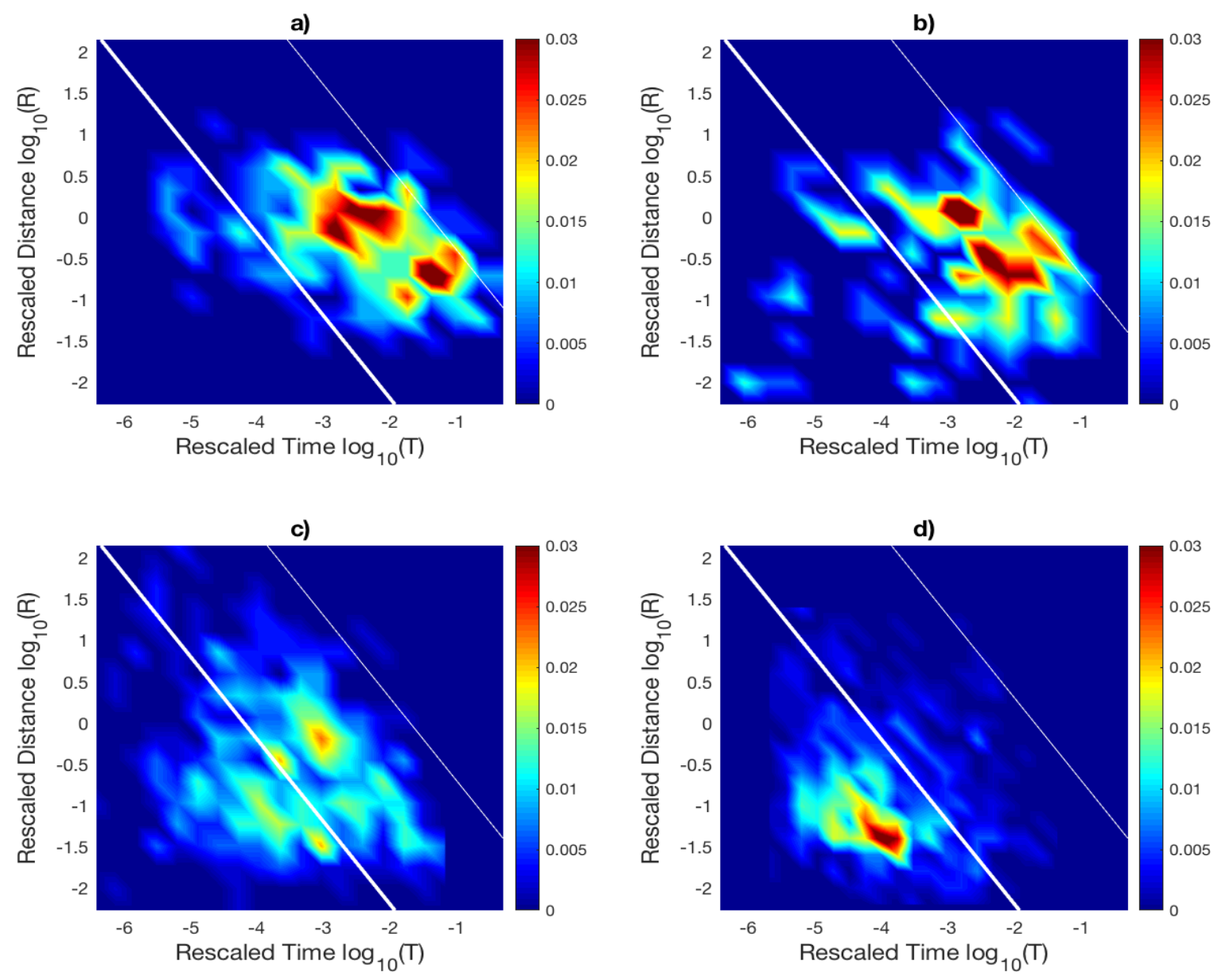

Figure 4-28: Joint NND distributions of the temporal and spatial inter-event distances (T,

$R$ ) for each investigated cluster. Bold white line indicates the threshold $\log _{10} \eta_{\text {thresh }}$ between tightly and loosely clustered components. Thin white line indicates the threshold $\log _{10} \eta_{\text {tect }}$ between the loosely clustered and deep-background. Colour bar reflects interevent distance occurrence probability. a) Rocky Mountain House cluster. b) Montney cluster 1. c) Montney cluster 2. d) Fox Creek cluster.

The largest 3 event families identified by the NND method for each cluster are plotted below with their associated sequence parameters. The decision to display only the largest 3 families here was simply to preserve space and prevent repetitive plotting; the reader is invited to review 
the full sets of sequences above (Figure 4-5, Figure 4-11, Figure 4-17 and Figure 4-23). In order to provide a more complete description, Table 4-22 presents the mean and median parameter values for all significant event families within each cluster. Several interesting comparative observations are worthy of note.

Perhaps the most obvious differences between event families are their relative sizes and frequencies of occurrence, which are also reflected in the contrasting joint NND distributions (Figure 4-28). The RMHC and MC1 are each composed of 6 significant sequences across 26year periods; these sequences are small, with the largest containing 5 and 8 events, respectively. It is possible that the larger magnitude mainshocks triggered some additional aftershocks whose magnitudes were below the threshold of detection. The MC2 is composed of 10 sequences over a 9-year period, the largest containing 17 events, while FCC is comprised of 16 sequences over a 6-year period, the largest containing 80 events. The mean and median values of the sequence size $\mathrm{N}$ and average leaf depth $\langle\mathrm{d}\rangle$ for all families underline the disparities in extent and frequencies of occurrence (Table 4-22, columns 4 and 5). Once again, very little tight clustering occurs within the RMHC and MC1, and what do transpire are smaller sequences (mean and median sizes fewer than 5 events). The MC2 contains comparatively moderate levels of clustering, with 2 larger sequences (Figure 4-31 clusters 1 and 2, 17 and 16 events) and the rest much smaller (mean and median sizes 7.6 and 5.5 events). Meanwhile, the FCC contains the largest sequences by far (Figure 4-32), with mean and median sizes greater than 20 events.

The similarities and differences between the sequences' structural characteristics within each cluster are quite striking. The RMHC is almost entirely composed of small bursts; these sequences have a relatively large mainshock and are followed by a few (4 or less) aftershocks (Figure 4-29). The mean and median values of the magnitude differential $\Delta \mathrm{M}$ are large while the topological normalized leaf depth $\delta$ and inverted branching number $B_{i}$ are small, highlighting the spray-like nature of the sequences (Table 4-22, columns 6, 7 and 10). The MC1 is comprised of a mixture of small bursts and swarms. Its mean and median $\Delta \mathrm{M}$ are slightly smaller and its topological parameters are larger than the RMHC's, demonstrating more swarmlike behavior and possibly some level of inter-event triggering. The MC2 is even more swarmlike than the MC1; it consists of a large swarm, a large burst and smaller sequences. The mean and median topological parameters are larger than both previous clusters while the magnitude 
differential is smaller. This signifies that the mainshocks are generally less distinct (apart from the large burst), the sequences are graphically deeper and there are more parent events and fewer leaves (end-nodes). The mean $\Delta \mathrm{M}$ is skewed somewhat by the large burst, which explains the disparity between it and the median value (0.27 vs. 0.1). The FCC is overwhelmingly swarm-like; its sequences are almost all chain-like in time (mean and median normalized leaf depth $\delta$ are very large) and consist of similarly sized events (mean and median $\Delta \mathrm{M}$ are small). Its $B_{i}$ values are similar to the $\mathrm{MC} 1$ and $\mathrm{MC} 2$. It is important to note that while the average leaf depth $\langle\mathrm{d}\rangle$ scales with the sequence size $\mathrm{N}$, both $\delta$ and $B_{i}$ do not. Therefore, the variations observed in these parameters between clusters are significant.

The spatial extents $\mathrm{A}$ and rates of decay $t_{d}$ are slightly more difficult to contextualize, partly because these parameters do tend to scale with $\mathrm{N}$. The sequences within the RMHC are quite consistent; they all cover small spatial areas and decay rapidly (within days) (Table 4-22, columns 8 and 9). The MC1 suffered from some improper location recording where several events were placed in the same spot, resulting in inaccurate area calculations. Its decay periods are variable; the two largest bursts both persist for over a month while the remaining smaller sequences degenerate within days. The MC2 is once again consistent apart from the large burst, which covers a large area and persists for several months. The remaining sequences are much more constrained spatiotemporally, including the largest swarm sequence, which explains the disparity between mean and median $\mathrm{A}$ and $t_{d}$ values. Finally, the FCC's swarms and swarmbursts are quite similar, reflected in the comparable mean and median A and $t_{d}$ values. The largest swarms cover spatial areas between $30-50 \mathrm{~km}^{2}$ and span approximately a month's time, while the smaller sequences cover $10 \mathrm{~km}^{2}$ or less and decay within 2 weeks. On the other hand, the lone burst sequence occupies a larger area and spans over half a year's time (Figure 4-23 and Table 4-15, cluster 4). 
a)
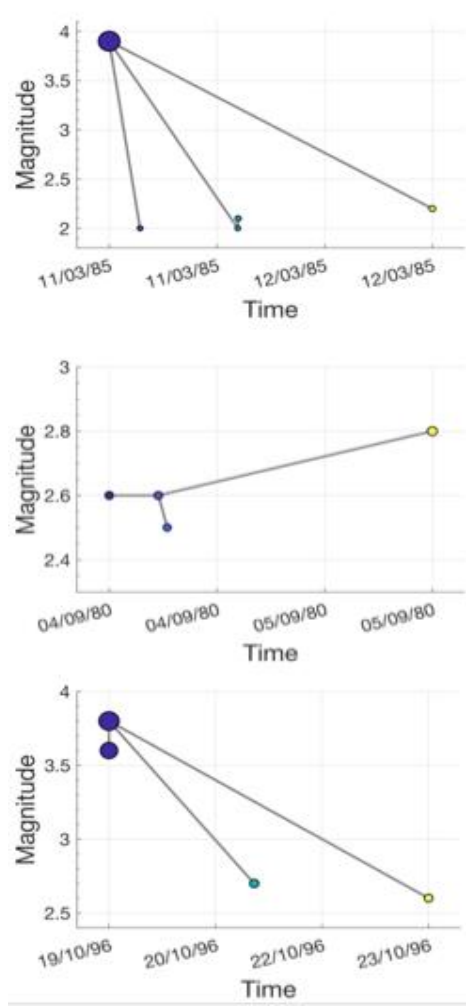

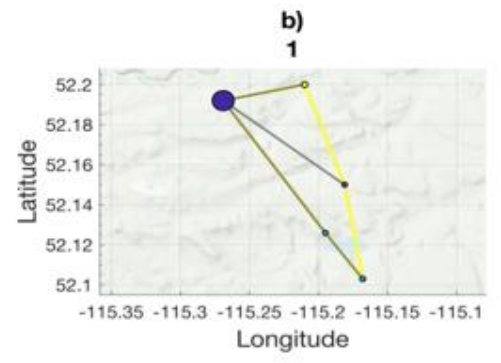

2

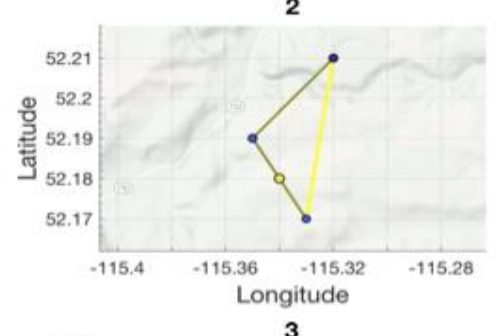

3

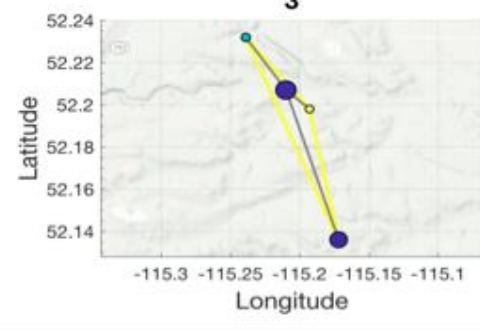

c)
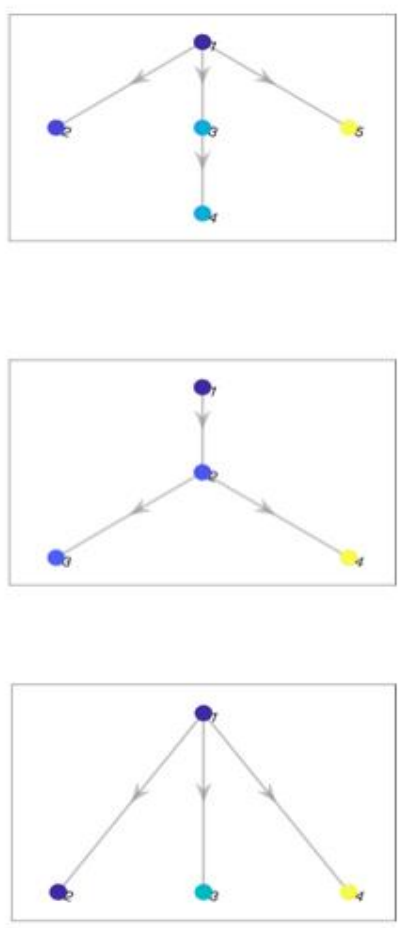

Figure 4-29: Largest 3 NND Event Families identified within the Rocky Mountain House cluster. a) Moment magnitude vs. Time in days. b) Spatial map. Yellow border outlines the hull area occupied by the sequence. c) Directed tree graph in dimensionless space.

Data points are coloured chronologically from darkest to lightest.

Table 4-18: Parameter sets for the largest 3 event families within the Rocky Mountain

House cluster. $N$ is the size of the sequence, $\langle d\rangle$ is the average leaf depth, $\delta$ is the normalized leaf depth, $B_{i}$ is the inverted branching number, $A$ is the spatial area, $t_{d}$ is the decay period, and $\Delta M$ is the magnitude differential.

\begin{tabular}{cccccccccc}
\hline Sequence & $\mathbf{N}$ & $\langle\boldsymbol{d}\rangle$ & $\boldsymbol{\delta}$ & $\boldsymbol{B}_{\boldsymbol{i}}$ & $\boldsymbol{A}\left(\mathbf{k m}^{2}\right)$ & $\boldsymbol{t}_{\boldsymbol{d}}(\mathbf{d a y s})$ & $\boldsymbol{\Delta M}$ & Class \\
\hline $\mathbf{1}$ & 5 & 1.33 & 0.60 & 0.50 & 25.98 & 1.26 & 1.70 & Burst \\
$\mathbf{2}$ & 4 & 2.00 & 1.00 & 0.67 & 3.79 & 0.75 & 0.20 & Unknown \\
$\mathbf{3}$ & 4 & 1.00 & 0.50 & 0.33 & 8.08 & 4.31 & 0.20 & Burst \\
\hline
\end{tabular}



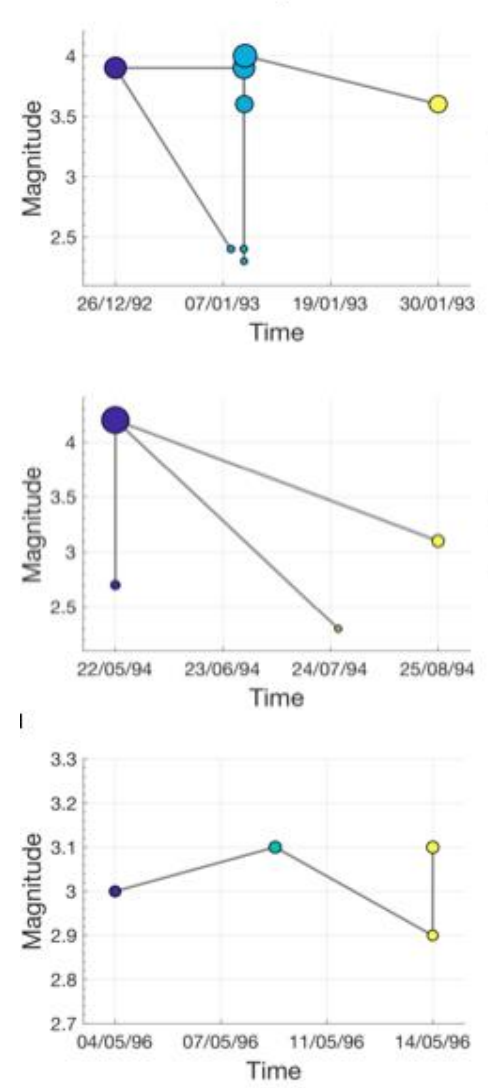

b)

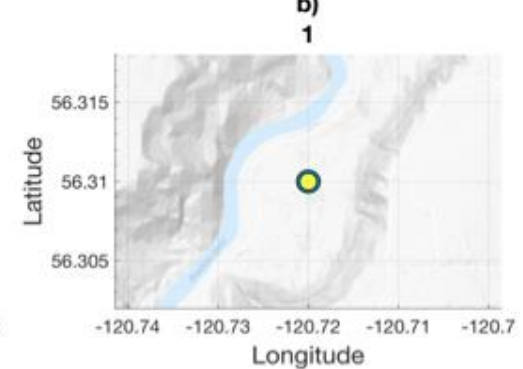

2

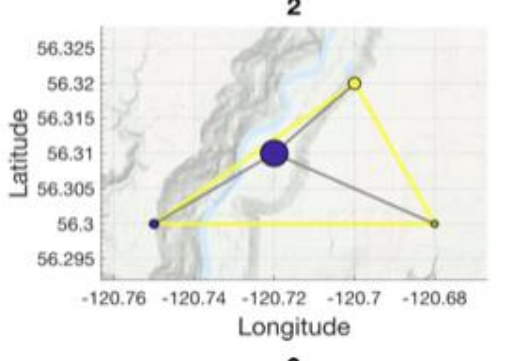

3

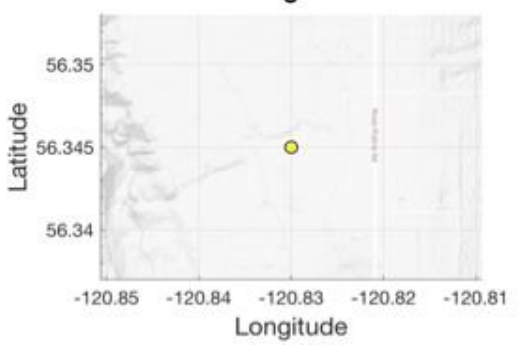

c)
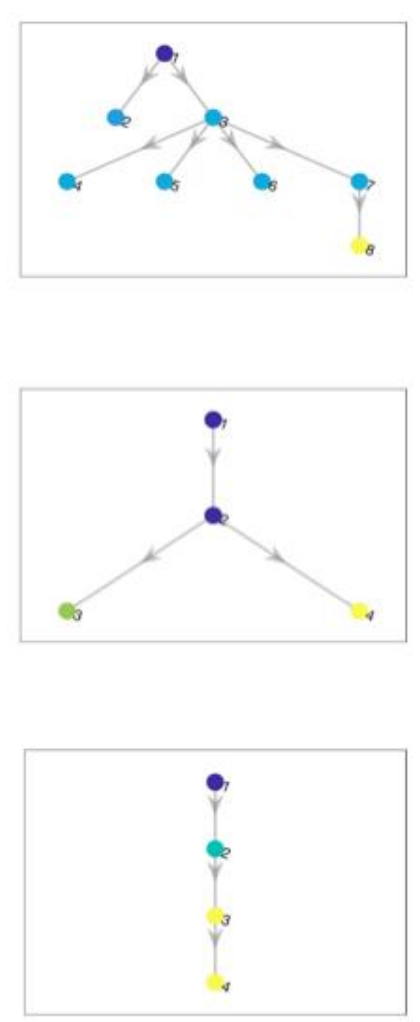

Figure 4-30: Largest 3 NND Event Families identified within the Montney cluster 1. See Figure 4-29 for a graphical description.

Table 4-19: Parameter sets for the largest 3 event families within the Montney cluster 1. See Table 4-18 for parameter descriptions.

\begin{tabular}{ccccccccc}
\hline Sequence & $\mathbf{N}$ & $\langle\boldsymbol{d}\rangle$ & $\boldsymbol{\delta}$ & $\boldsymbol{B}_{\boldsymbol{i}}$ & $\boldsymbol{A}\left(\mathbf{k m}^{2}\right)$ & $\boldsymbol{t}_{\boldsymbol{d}}$ (days) & $\boldsymbol{\Delta M}$ & Class \\
\hline $\mathbf{1}$ & 8 & 2.00 & 0.71 & 0.43 & 0.00 & 34.88 & 0.10 & Burst \\
$\mathbf{2}$ & 4 & 2.00 & 1.00 & 0.67 & 4.80 & 94.82 & 1.10 & Burst \\
$\mathbf{3}$ & 4 & 3.00 & 1.50 & 1.00 & 0.00 & 10.14 & 0.00 & Swarm \\
\hline
\end{tabular}


a)
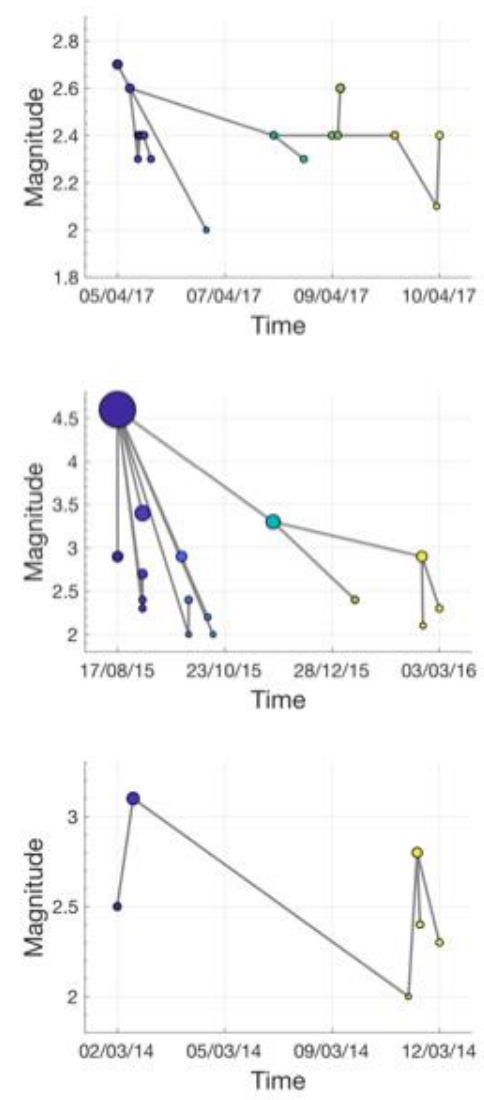
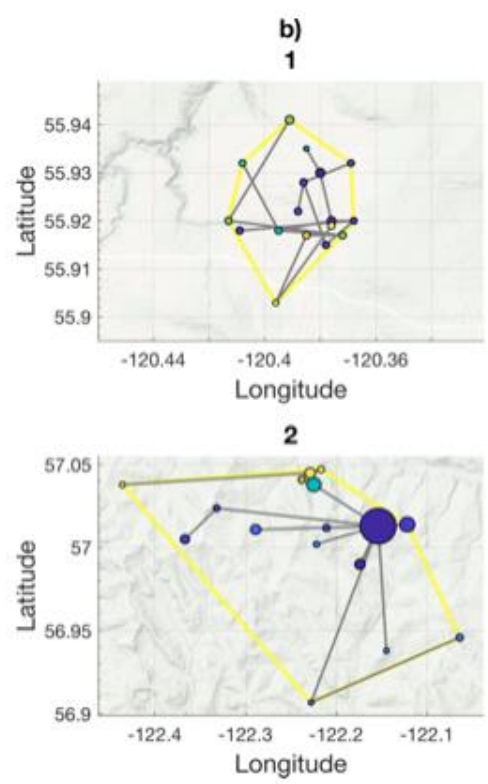

3

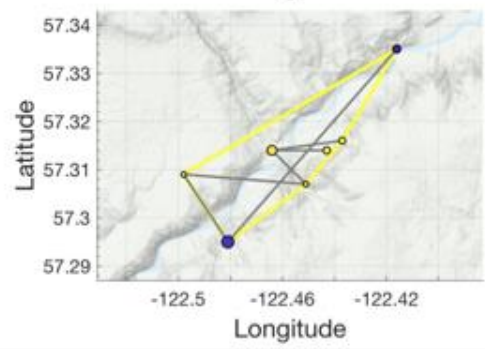

c)
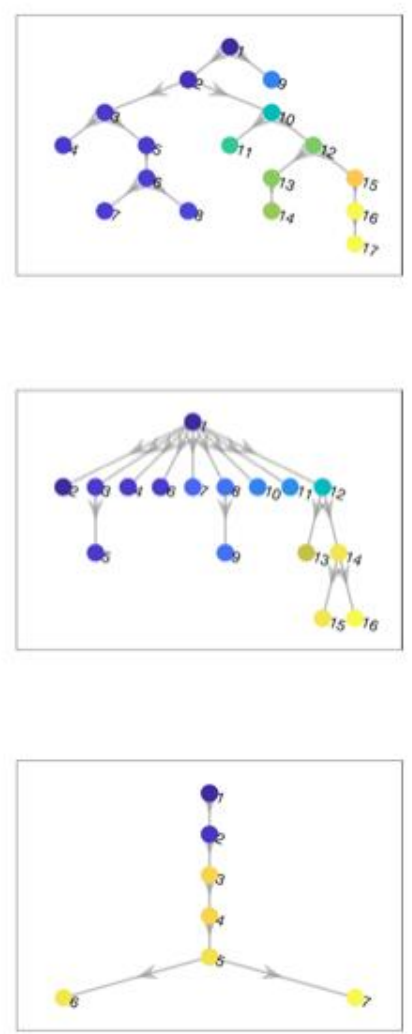

Figure 4-31: Largest 3 NND Event Families identified within the Montney cluster 2. See Figure 4-29 for a graphical description.

Table 4-20: Parameter sets for the largest 3 event families within the Montney cluster 2.

See Table 4-18 for parameter descriptions.

\begin{tabular}{cccccccccc}
\hline Sequence & $\mathbf{N}$ & $\langle\boldsymbol{d}\rangle$ & $\boldsymbol{\delta}$ & $\boldsymbol{B}_{\boldsymbol{i}}$ & $\boldsymbol{A}\left(\mathbf{k m}^{2}\right)$ & $\boldsymbol{t}_{\boldsymbol{d}}$ (days) & $\boldsymbol{\Delta M}$ & Class \\
\hline $\mathbf{1}$ & 17 & 4.00 & 0.97 & 0.62 & 7.41 & 5.44 & 0.10 & Swarm \\
$\mathbf{2}$ & 16 & 1.64 & 0.41 & 0.33 & 194.01 & 198.48 & 1.20 & Burst \\
$\mathbf{3}$ & 7 & 5.00 & 1.89 & 0.83 & 6.97 & 9.87 & 0.30 & Swarm \\
\hline
\end{tabular}


a)
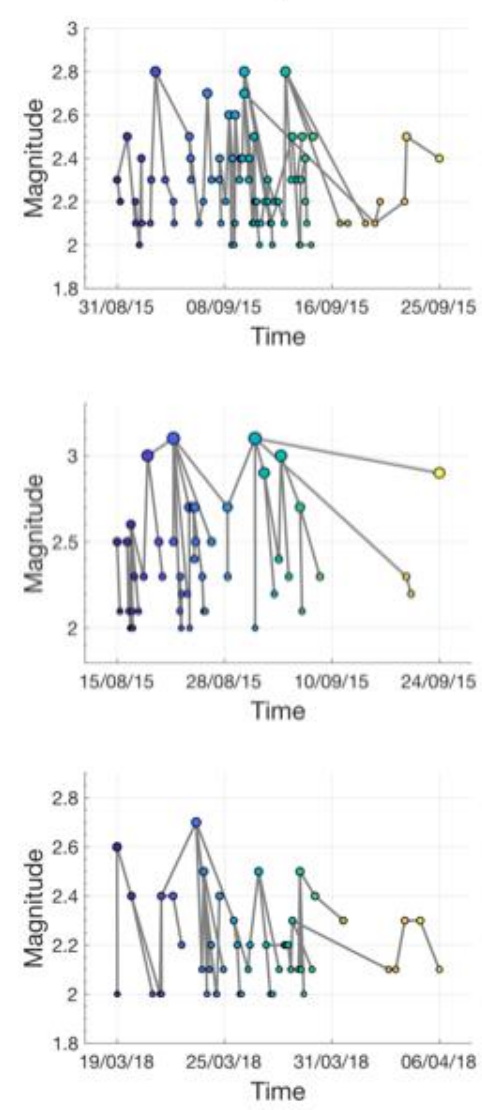

b)

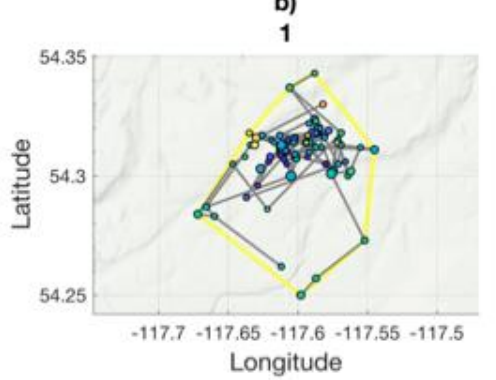

2

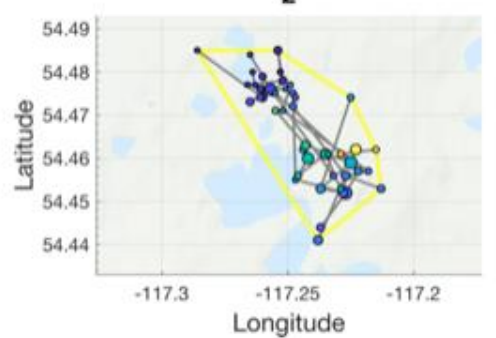

3

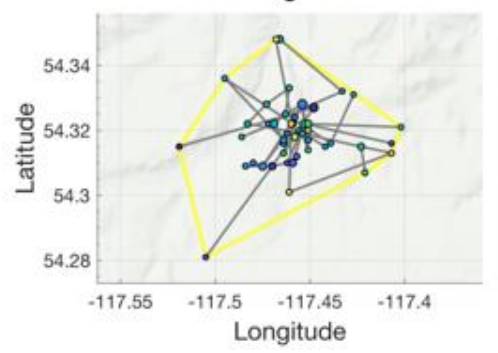

c)
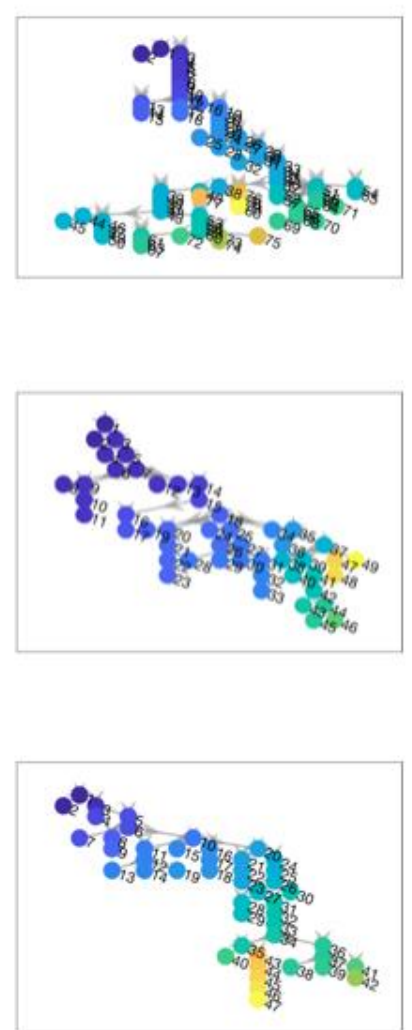

Figure 4-32: Largest 3 NND Event Families identified within the Fox Creek cluster. See Figure 4-29 for a graphical description.

Table 4-21: Parameter sets for the largest 3 event families within the Fox Creek cluster. See Table 4-18 for parameter descriptions.

$\begin{array}{llllllllll}\text { Sequence } & \mathbf{N} & \langle d\rangle & \boldsymbol{\delta} & \boldsymbol{B}_{i} & \boldsymbol{A}\left(\mathrm{km}^{2}\right) & \boldsymbol{t}_{\boldsymbol{d}} \text { (days) } & \Delta M & \text { Class }\end{array}$

\begin{tabular}{lllllllll}
\hline $\mathbf{1}$ & 80 & 28.43 & 3.18 & 0.75 & 50.40 & 24.47 & 0.00 & Swarm \\
$\mathbf{2}$ & 49 & 7.68 & 1.10 & 0.50 & 11.65 & 40.03 & 0.00 & Swarm \\
$\mathbf{3}$ & 47 & 9.18 & 1.34 & 0.65 & 31.42 & 18.06 & 0.10 & Swarm \\
\hline
\end{tabular}


Table 4-22: Mean and median parameter values for all significant event families within each investigated cluster. See Table 4-18 for parameter descriptions.

\begin{tabular}{|c|c|c|c|c|c|c|c|c|c|}
\hline Cluster & $\begin{array}{c}\text { Number of } \\
\text { Families }\end{array}$ & & $\mathbf{N}$ & $\langle d\rangle$ & $\delta$ & $\boldsymbol{B}_{i}$ & $\begin{array}{c}A \\
\left(\mathbf{k m}^{2}\right)\end{array}$ & $\begin{array}{c}t_{d} \\
\text { (days) }\end{array}$ & $\Delta M$ \\
\hline \multirow{2}{*}{ RMHC } & \multirow{2}{*}{6} & Mean & 3.67 & 1.22 & 0.64 & 0.50 & 8.40 & 1.33 & 0.57 \\
\hline & & Median & 3.5 & 1 & 0.58 & 0.5 & 5.93 & 1.00 & 0.25 \\
\hline \multirow{2}{*}{ MC1 } & \multirow{2}{*}{6} & Mean & 4.17 & 1.83 & 0.92 & 0.68 & 23.45 & 23.60 & 0.35 \\
\hline & & Median & 3.5 & 2 & 0.855 & 0.58 & 4.42 & 5.52 & 0.25 \\
\hline \multirow{2}{*}{ MC2 } & \multirow{2}{*}{10} & Mean & 7.60 & 2.76 & 1.08 & 0.67 & 26.33 & 24.83 & 0.27 \\
\hline & & Median & 5.5 & 3 & 1.125 & 0.71 & 7.19 & 5.23 & 0.1 \\
\hline \multirow{2}{*}{ FCC } & \multirow{2}{*}{16} & Mean & 27.13 & 7.60 & 1.54 & 0.66 & 20.97 & 32.09 & 0.22 \\
\hline & & & & 6.10 & 127 & 065 & 1716 & 1505 & O 1 \\
\hline
\end{tabular}

Based on the observations made above, several potential conclusions may be drawn regarding the clustering within the WCSB. First, wherever fluid injection appears to be the seismogenic mechanism, either through wastewater disposal or $\mathrm{HF}$ (i.e. the $\mathrm{MC} 1, \mathrm{MC} 2$ and $\mathrm{FCC}$ ), the tight clustering appears more swarm-like (reminiscent of ductile failure). This makes sense intuitively, as injected fluid is capable of diffusing through rock networks and destabilizing neighboring fault structures. The lone case of fluid extraction-related seismicity, the RMHC, resulted in the solely burst-like sequencing of brittle failure. Second, HF operations appear to trigger greater, even more swarm-like behavior than wastewater injection, as the MC2 and FCC contain higher levels of swarm seismicity than does the MC1. This may be a consequence of the differences in injection volume and rate between cases, as well as the horizontal orientation of many new HF wells, which is capable of forcing fluid through a much larger volume of rock in the short term (King, 2010; Smith \& Montgomery, 2015). Third, HF is also capable of triggering large aftershock sequences (Figure 4-31, cluster 2; Figure 4-23, cluster 4), where the 
migrating fluid may be traveling into the crystalline basement and interacting with critically stressed faults, similar to the triggering mechanism attributed to wastewater disposal. Fourth, these large aftershock sequences result in earthquakes that span much larger spaces and time frames than do the swarms of comparable sequence size. The swarms within the MC2 and FCC are almost all tightly constrained in space and time relative to their size, which may correlate with the spatial and temporal extent of their associated stimulating HF operations. This also suggests that the two types of clusters are caused by different mechanisms; the bursts by fluid intermingling with critically oriented faults resulting in a large event, which then triggers multiple offspring events in a conventional aftershock manner, versus the swarms where no distinct mainshock is present yet multiple offspring continue to transpire as the pumped fluid repeatedly disturbs nearby faults (Schultz et al., 2015c; Bao \& Eaton, 2016; Eaton, 2018). A possible example of swarms being directly related to HF activity is the notable resemblance between the structures of the identified swarms in the FCC and the largest sequence in the MC2 near Dawson Creek (Figure 4-31, cluster 1). Visually, the Dawson Creek swarm is a smallerscale version of the FCC sequences (Figure 4-32); it is a chain-like sequence of comparably sized events that decays relatively quickly. It is possible that the likenesses in the fundamental structuring of these clusters may be reflective of their shared triggering mechanism (HF). The disparities in their size and scope may be due to factors such as different pumping rates/pressures/times or total volume of fluid injected, as well as local geologic factors.

Figure 4-33 presents the ETAS model fits to each cluster in original time. The corresponding ETAS parameters are summarized in Table 4-23. The model successfully converged for all four clusters, but with varying qualities of fit (QOF) (Table 4-23, last column). The RMHC (panel a) is by far the worst fit; the MC1 (panel b) fit is fair, the MC2 (panel c) is good while the FCC (panel $\mathrm{d}, \mu \approx 0$ ) is very good. It is somewhat difficult to assess and compare parameter values when the model may not be accurately representing the rate in some cases (i.e. the RMHC and MC1). The type of seismicity within each cluster, as described by the NND model, may provide some explanation for the vastly differing QOFs. The FCC is composed of large, distinct clustering sequences that are easily distinguishable across time. The ETAS model appears to be able to correctly model these individual spikes in the temporal rate quite easily. Conversely, the RMHC is composed of a loosely clustered type of seismicity that produces few clearly defined sequences and mainly acts as an elevated background rate. Consequently, the ETAS model 
encounters difficulty replicating the gradually increasing rate with an absence of "aftershock" sequences. Indeed, the steady RMHC rate may be more suitably modeled by the background rate parameter $\mu$ without the aftershock kernel. The improved QOF of the MC2 over the MC1 could be further evidence that the ETAS model requires defined sequences with which to optimize its fit, although the MC1 dataset may be too small for a realistic comparison.

It is interesting that the ETAS model is capable of accurately capturing swarms in addition to the aftershock sequences for which it was intended. The swarms within both the MC2 and FCC do not have distinct mainshocks and are instead composed of similarly sized events (see discussion above). This is, in fact, reflected in the optimized ETAS $\alpha$ parameter, which governs the dependence on magnitude in the generation of further "aftershocks" (Table 4-23, column 8). The $\alpha$ parameters are low for both clusters, indicating that the ETAS model is correctly identifying the persistent nature of the swarm sequences, which continue to produce offspring despite the lack of an obvious mainshock. 
a)

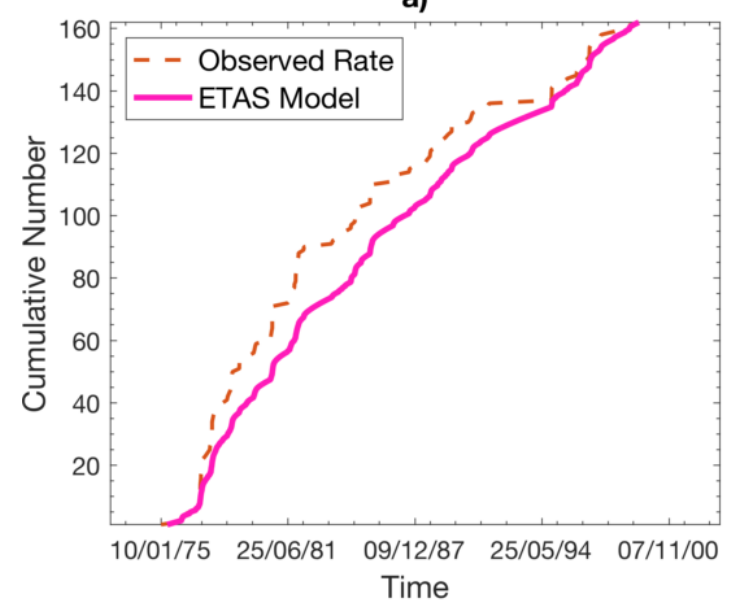

c)

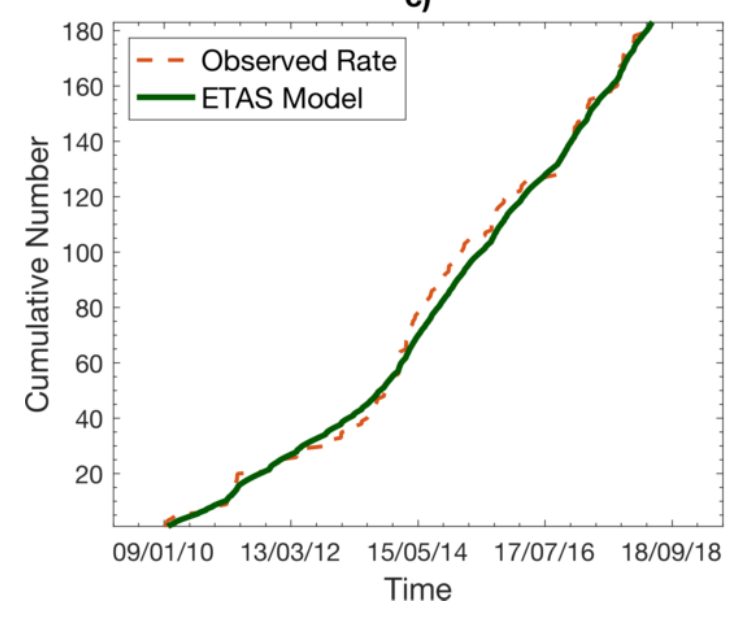

b)

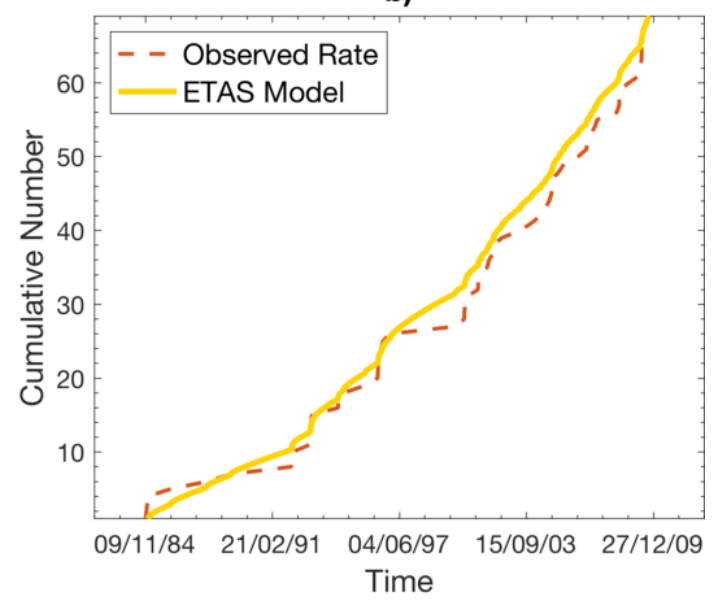

d)

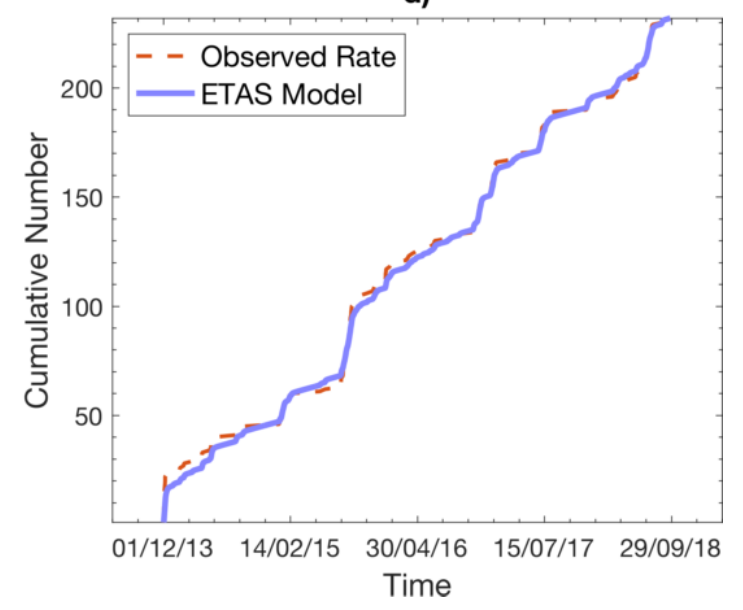

Figure 4-33: ETAS Models in original time for each investigated cluster. Dashed orange lines are the observed cumulative rates of seismicity. Solid coloured lines are the optimized models. a) Rocky Mountain House cluster. b) Montney cluster 1. c) Montney cluster 2. d) Fox Creek cluster. 
Table 4-23: ETAS model parameters for each investigated cluster. $\mathrm{N}$ is the number of events modeled, $\mu$ is the constant background rate, $K$ is the aftershock productivity, $c$ is the temporal offset, $p$ is the exponential decay, $\alpha$ is the magnitude-aftershock dependence, and QOF is the quality of the model fit.

\begin{tabular}{ccccccccc}
\hline Cluster & $\mathbf{N}$ & $\boldsymbol{M}_{\boldsymbol{c}}$ & $\boldsymbol{\mu}$ & $\boldsymbol{K}$ & $\boldsymbol{c}$ & $\boldsymbol{p}$ & $\boldsymbol{\alpha}$ & QOF \\
\hline RMHC & 162 & 2.3 & 0.0047 & 0.26 & 0.24 & 1.06 & 0.76 & 0.072 \\
MC1 & 69 & 2.7 & 0.0033 & 3.09 & 0.0019 & 0.81 & 0.84 & 0.042 \\
MC2 & 183 & 2.5 & 0.016 & 1.0 & 0.022 & 0.84 & 0.39 & 0.020 \\
FCC & 227 & 2.5 & 0.00006 & 0.50 & 0.58 & 1.27 & 0.38 & 0.012 \\
\hline
\end{tabular}

Finally, within the FCC, both a better fit and a realistic convergence of the ETAS model are achieved by constraining the background rate to nearly zero (Figure 4-25 versus Figure 4-26). When the $\mu$ parameter was similarly fixed on a trial basis for the other clusters, either the QOF was worse or the model did not converge. This makes further sense intuitively, as the seismicity in those areas was largely made up of an elevated background with few significant sequences, and so assuming an absent background rate would be inaccurate. The high-quality fit of the ETAS model to the FCC (regardless of the $\mu \approx 0$ constraint), compared to the fits of the other clusters, again provides verification that the model performs better on a dataset containing separable, tightly connected sequences. For seismic clusters resembling an elevated background rate, on the other hand, the model may not be able to predict what events are or are not part of a sequence. 


\section{Chapter 5}

\section{Conclusions and Future Work}

The alarming rise in spatiotemporally clustered seismicity caused by anthropogenic, energyrelated activities within the Western Canada Sedimentary Basin (WCSB) and elsewhere around the world has prompted much recent scientific inquiry (Schoenball et al., 2015; Skoumal et al., 2015; Atkinson et al., 2016; Petersen et al., 2016; McClure et al., 2017; McGarr \& Barbour, 2017; Keranen \& Weingarten, 2018; Pawley et al., 2018; Brudzinski \& Kozłowska, 2019). Improved understanding of the related triggering processes, source mechanisms, geologic susceptibilities, resulting clustering patterns and statistics will continue to improve the efficiency of mitigation and prevention strategies and allow for more a rapid identification of induced activity (Llenos \& Michael, 2013; Schultz et al., 2016, 2018; Zaliapin \& Ben-Zion, 2016; Atkinson, 2017; Schoenball \& Ellsworth, 2017; Kao et al., 2018). This study aimed to characterize the past and present seismicity recorded within the WCSB using statistical models, with the goals of highlighting fundamental differences between the induced and natural activity and providing insight into the clustering properties of earthquakes stimulated by specific human endeavors. Hydraulic fracturing (HF) operations appear capable of triggering seismicity that is measurably distinct from both tectonic activity and past earthquakes induced by conventional hydrocarbon production and co-produced wastewater disposal.

\subsection{Discussion of Results}

\subsubsection{Regional Analysis of Seismicity within the WCSB (Chapter 3)}

In Chapter 3, a regional study analyzing space-time inter-event nearest-neighbour distances was conducted for all the seismicity recorded across Alberta and eastern B.C. over time, beginning from the first observed instance of induced activity in 1975 up to the nearly present HF-related activity in 2018. Analysis over the entire time frame (1975-2018) revealed the existence of a trimodal inter-event distance distribution, where events generally appeared to either transpire very closely together in space and time (within the tightly clustered mode), moderately close together (within the loosely clustered mode) or else were distributed according to a stationary Poisson process (the deep background). Further, these distinct earthquake subpopulations were observed to be stable for cut-off magnitudes up to the regional completeness level $\left(M_{c} \approx 3.0\right.$, 
Appendix A). Analysis over separated time intervals demonstrated that a vastly disproportionate increase in both the loosely and tightly clustered earthquake components occurred between 1975-2009 and 2010-2018, where the first interval contained seismicity largely predating the broad-scale implementation of HF technology and the second interval contained events that occurred during the implementation and operational stages. Based on the even spatial distribution of the third mode occurring at large inter-event distances (resembling a Poisson point process), as well as its decreased proportion over the recent (shorter) time frame, this mode was inferred to be representative of the natural tectonic background seismicity within the region. These events contrasted measurably with the highly localized spatial distributions of the remaining two event subpopulations occurring at smaller inter-event distance values, which also held dominant mixing proportions (increased rates of activity) over recent times. I posited that the majority of past and present induced activity occurred within these two modes, and that their increasing populations within the region pointed to the growing usage of HF technology and could not be fully explained by the improvement in seismic network resolution.

Many other regional studies on induced seismicity in active areas describe a general elevation in the background rate during and immediately after potential earthquake-inducing processes such as geothermal energy production, wastewater disposal, hydraulic fracturing etc. (Lombardi et al., 2010; Llenos \& Michael, 2013; Schoenball et al., 2015; Maghsoudi et al., 2016; Zaliapin \& Ben-Zion, 2016; Schoenball \& Ellsworth, 2017; Martínez-Garzón et al., 2018). In Chapter 3, as well as in Chapter 4 where several prominent clusters were investigated individually, it was found that the loosely clustered rate was substantially elevated near areas of human activity, which in turn led to a higher probability of event-sequence generation (i.e. tight clustering). It should be emphasized that this loosely clustered "background" seismicity deviated from what may have been the region's natural tectonic dynamics (the deep-background), in that its interevent space-time distances were observably lower and the population therefore appeared more clustered than a typical Poisson process. It is possible that this is the case in other regions, and that the smaller proportion of natural, tectonic events is overshadowed in the NND distributions due to the significantly higher levels of loosely clustered and tightly clustered events.

For example, Schoenball et al. (2015) utilized the NND method to demonstrate that distinguishing between induced and natural activity near the Coso Geothermal Field (CGF) in 
southern California required a multi-dimensional analysis of earthquake space, time and magnitude occurrence. When applied separately, the one-dimensional constituents did not appear to have discriminatory power. The study divided the region surrounding the CGF such that Study Area A-B contained events within Study Area A but outside of Study Area B (Figure 5-1). A-B contained natural seismicity stemming from tectonic and magmatic sources while activity within B was inferred as primarily induced (stimulated by geothermal energy production). The dataset was further divided temporally into roughly 7-year intervals (Figure 5-2) where the first interval covered the pre-production period and the remaining four covered the co-production period. The distributions look similar during pre-production (Figure 5-2, panels a \& f); both A-B and B are comprised of a large tightly clustered mode and a smaller background component that exists far away from the separation threshold, reminiscent of the deep-background mode observed in the present study. The remaining time frames are quite different. A-B retains its shape during co-production while B changes; its tightly clustered component shrinks and a background mode appears much closer to the threshold. This background mode in Figure 5-2, panels g-j, may be comparable to the loosely clustered component observed within the WCSB. The comparison makes sense intuitively; the area A-B is larger and thus more likely to contain a larger proportion of Poisson-like seismicity whereas area B is spatially constrained around a known cluster of events (similar to the spatial distribution of events shown in Figure 3-6; the loosely clustered and tightly clustered events dominate known areas of induced activity while the deep-background is spread across the map). Schoenball et al. (2015) correctly pointed out that the location of the background mode is dependent on the seismic rate per area; during co-production, area B saw an increased rate and thus its background mode "shifted" closer to the clustered domain. I propose that this shift in background location may be the manifestation of a measurably distinct earthquake subpopulation that occurs at smaller inter-event space-time distances compared to the natural background observed in area A-B. 


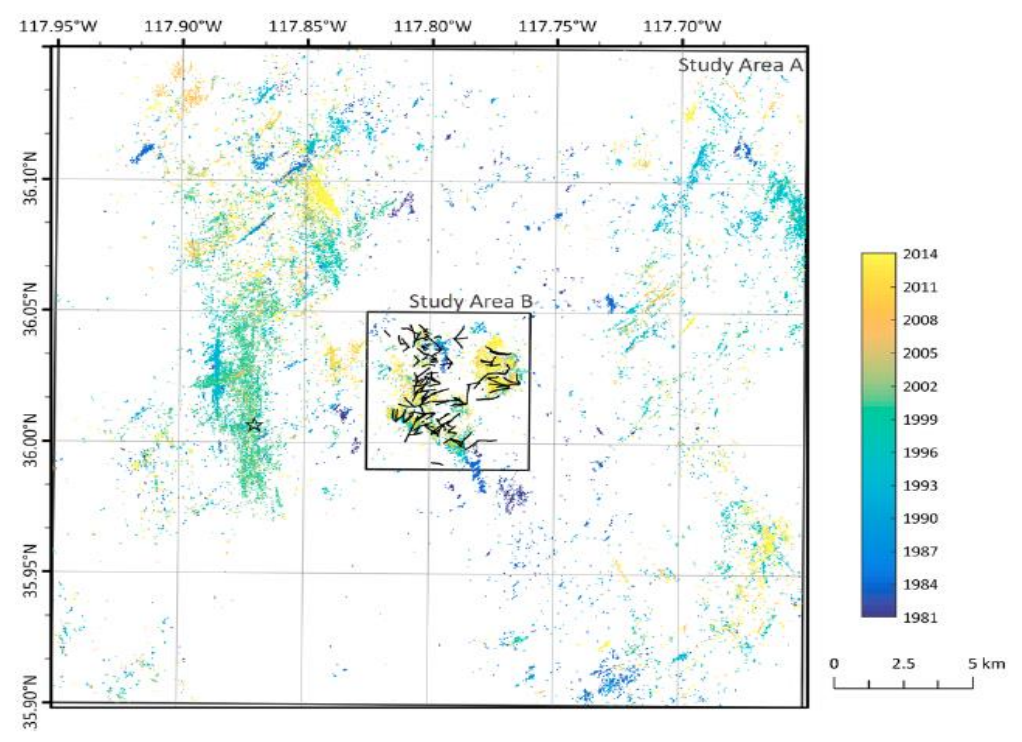

Figure 5-1: Seismicity surrounding the Coso Geothermal Field from 1981-2013. Colour corresponds to time with younger seismicity projected on top of older seismicity. Wellbore trajectories are shown as black lines. From Schoenball et al. (2015).
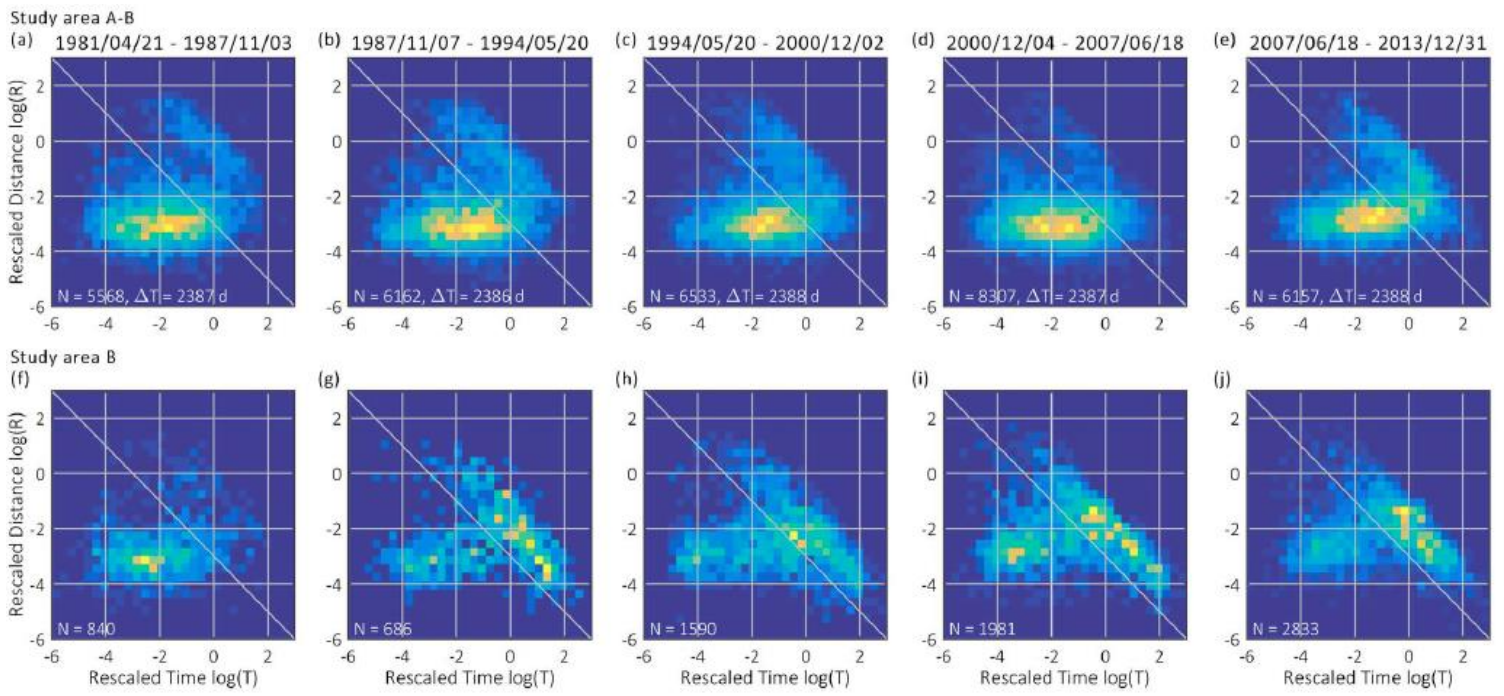

Figure 5-2: Joint (T, R) histograms for the Coso Geothermal Field and surrounding area.

Study areas (top row) A-B and (bottom row) B, for different time periods of the same length. The first time interval (left-hand side) is mostly pre-production, whereas the later intervals are co-production. The colour scale is normalized for each plot. From Schoenball et al. (2015). 
Another interesting property of the A-B study area is that it maintains a large tightly clustered mode despite the absence of a corresponding loosely clustered component. In the analysis of the WCSB in Chapter 3, the loosely clustered mode appeared much more likely than the deepbackground to initiate clustering, particularly significant clustering. The distributions in panels a-f of Figure 5-2 therefore suggest either that the small population of (possibly deep-) background events is capable of stimulating vast amounts of tightly clustered activity, or that external factors are contributing to the tight clustering. A likely cause is the volcanic setting within which the CGF operates; flowing magma has been observed to trigger large amounts of seismicity in the form of swarms with potentially higher $b$-values (Hill et al., 1990; Wyss et al., 2005; Bridges \& Gao, 2006; Schoenball et al., 2015). A similar phenomena was explored in Chapter 4, where the seismic activity near Fox Creek, Alberta resulted in a likewise NND distribution, with vast amounts of tight clustering and correspondingly few background events. The surrounding hydraulic fracturing (HF) activity was inferred as a major external factor, possibly contributing much in the same manner as the magmatic forces near the CGF. HF involves the injection of fluid at high pressures to create micro-fractures within a source rock; this fluid is capable of diffusing through the subsurface along preexisting channels, elastically altering stress conditions and (repeatedly) disturbing faults via fluid pressurization (Rutledge et al., 2004; Atkinson et al., 2016; Bao \& Eaton, 2016; Kettlety et al., 2019) (see Chapters 4.3 and 4.4).

A second example of multiple possible background modes occurred in a study by Vasylkivska \& Huerta (2017), who used the NND method to study Oklahoma's rapidly increasing seismicity rate due to the large-scale wastewater injection prevalent across the mid-western United States (Ellsworth, 2013; Llenos \& Michael, 2013; van der Elst et al., 2013). For example, from 19702009, there was an average of two M3+ events recorded per year; this number jumped to between 500-800 M3+ events per year during 2014 to 2016 (Vasylkivska \& Huerta, 2017). The spatial distribution of events also changed markedly since 2013, with the vast majority of recent events occurring in the northern half of Oklahoma. In the study by Vasylkivska \& Huerta (2017), the NND method was applied to the entire catalogue (Figure 5-3, panel a), as well as to separated time intervals: 1970-2009, mainly natural seismicity (panel b); 2010-2013 and 20142016 (panels c \& d), both chiefly induced activity. The distribution of the entire catalogue (panel a) is heavily influenced by the co-injection time periods (panels c \& d), all featuring a 
dominant (possibly clustered) central mode and a much smaller background mode. The preinjection interval, however, shows stark contrast (panel b). A background mode is visible at very large inter-event times and distances, highlighted by the white ellipse, and appears very similar to the deep-background component in the WCSB. The low tectonic earthquake rate common to both regions is a likely reason for the deep-backgrounds' dominance before the onset of induced activity and subsequent disappearance once the higher induced rates overtake the distributions.
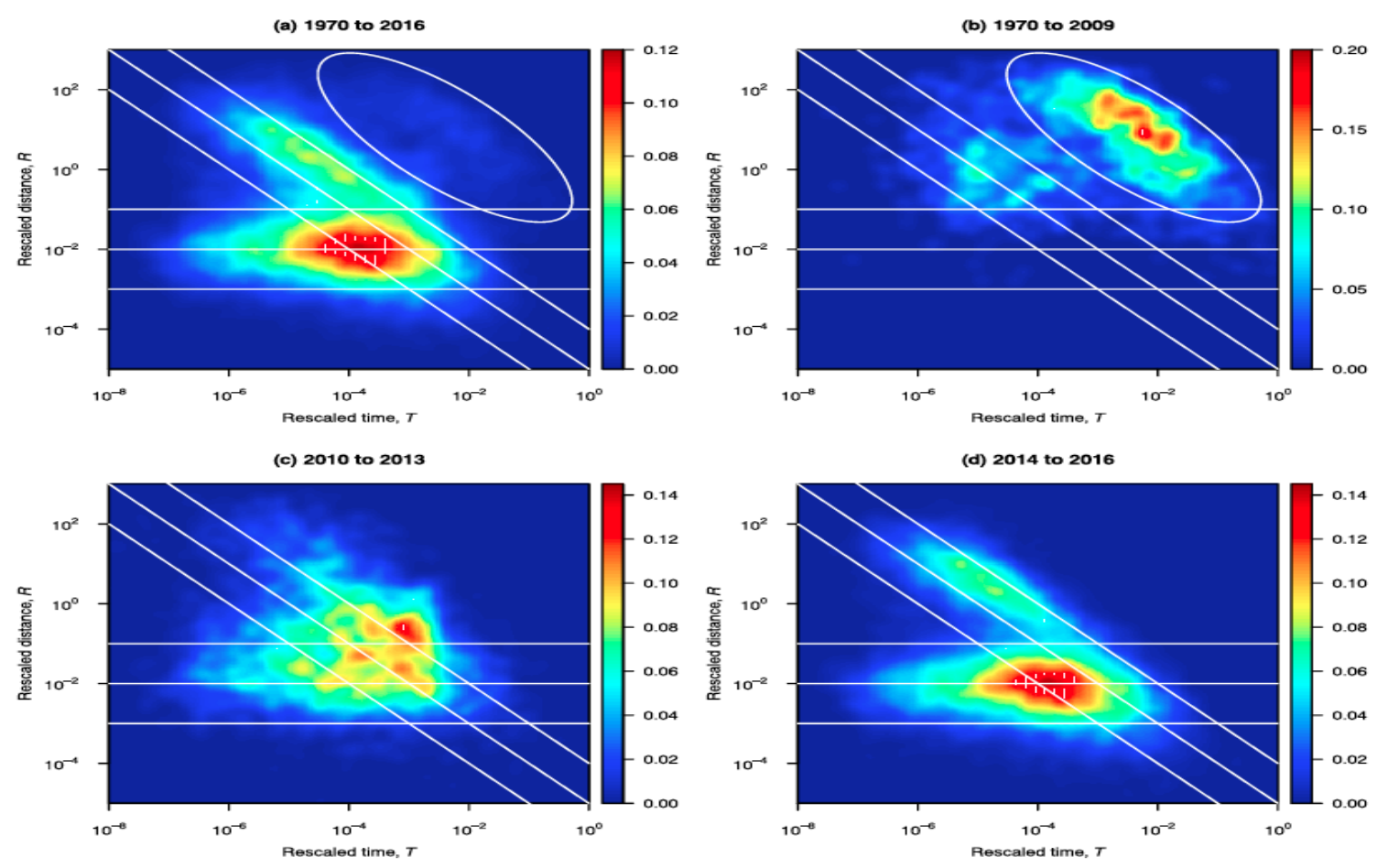

Figure 5-3: Joint (T, R) distributions for Oklahoma across different time periods. a) Entire catalogue (1970-2016). b) Pre injection-related seismicity (1970-2009). (c, d) Injection-related seismicity (2010-2013, 2014-2016). Ellipses highlight the pre injectionrelated background. Diagonal lines represent constant $\log _{10} \eta$ values and horizontal lines represent constant $\log _{10} R$ values. From Vasylkivska \& Huerta (2017).

Based on the results of the investigation in Chapter 3 and the corroborating evidence from other studies shown here, I posit that deep-background events should be regarded as a noteworthy element of the larger discussion of induced seismicity. They may provide an illustration of the 
statistical difference between natural and induced "background" earthquakes, both in quiescent and active regions alike.

\subsubsection{Investigation of Seismic Clustering within the WCSB (Chapter 4)}

In Chapter 4, a case-by-case investigation of four instances of induced seismic clustering within the WCSB was conducted utilizing the Gutenberg-Richter (GR) relation, the NearestNeighbour Distance (NND) method and the Epidemic Type Aftershock Sequence (ETAS) model. It was determined that the seismicity triggered by conventional gas extraction near Rocky Mountain House (the RMHC) and the wastewater disposal-related earthquakes near Fort St. John (the MC1) primarily manifested as discrete events, loosely clustered in space and time, that occupied the middle mode of the inter-event NND distributions. These events were characterized by low-to-moderate GR $b$-values and poorer fits to the ETAS model with a high sensitivity to magnitude for the generation of aftershocks. The few tightly clustered event sequences that did transpire were small and decayed rapidly. In the case of the RMHC, the sequences were solely burst-like in nature with relatively large mainshocks followed by a few small aftershocks, while the $\mathrm{MC1}$ contained a mixture of smaller burst and swarm sequences. The more recent clustering along the Montney formation (the MC2) also occupied the loosely clustered domain, but contained an additional mode within the tightly clustered domain. These earthquakes were characterized by a slight increase in $b$-value from the previous period and were better fit by the ETAS model, with a reduced magnitude-sensitivity parameter. More event sequences were identified over this period, which were larger and more swarm-like in nature. The addition of several new seismic stations across the Montney undoubtedly had an effect on these results, but an analysis of the occurrence rates between the MC1 and MC2 illustrated a disparate increase in tightly clustered versus loosely clustered activity, which could not be explained by the enhanced network coverage. Finally, near Fox Creek (the FCC), substantial HF-related activity transpired almost entirely within the clustered domain, in stark contrast with the previous clusters. These earthquakes formed a very steep frequency-magnitude distribution with a high $b$-value and fit the ETAS model exceptionally well, particularly when the background rate parameter was constrained to nearly zero, with a low magnitude sensitivity comparable to the MC2. The many detected event sequences were large, distinctly separable and overwhelmingly swarm-like. 
Some of the key observations that were made in Chapter 3 were also corroborated in Chapter 4 . Firstly, the claim that induced seismic activity appeared to occupy a specific subset of the interevent distance space, namely the loosely and tightly clustered modes, was verified. Secondly, the loosely clustered subpopulation was posited to be a consistent feature of induced seismicity independent of specific triggering mechanisms and thirdly, the sudden appearance of a tightly clustered mode in the regional distributions was asserted to be at least partially attributable to the recent application of HF for the development of unconventional resources. These claims were also endorsed by the cluster analyses, which showed that non HF-related induced activity (the RMHC and MC1) manifested mainly in the loosely clustered mode of the inter-event distance distribution, while the recent HF-triggered seismicity (the MC2 and FCC) occupied both the loosely clustered and a significant portion of the tightly clustered domain.

\subsection{Future Work}

The analyses and techniques presented in this work could be expanded upon and enhanced in several ways. A fundamental issue with the models used here are their dependence on sampling statistics, which generally vary in their representative accuracy and precision based on sample size and standard deviation. While the associated uncertainties for the maximum likelihood estimations of the GR and ETAS parameters can be quantified (though the ETAS parameter errors computed in this study were unstable and hence removed; this should be addressed in future work), the NND calculations are less well constrained. The decision to apply a magnitude cut-off $\left(M_{0}\right)$ below the completeness level for the analysis of nearest-neighbour distances is a justifiable one, as both Zaliapin \& Ben-Zion (2013a) and sensitivity tests conducted here (Appendixes A and B) show that results are stable with respect to the magnitude cut-off up to a certain point. Nevertheless, lowering the cut-off magnitude below completeness in order to enrich sampling undeniably introduces some artifacts into the investigation. Zaliapin $\&$ Ben-Zion (2013a) present a useful tool called the $\Delta$-analysis to assess the dependence of the clustered earthquake subpopulation on the chosen cut-off magnitude. Briefly, the $\Delta$-analysis replicates the traditional NND analysis but only considers mainshocks whose magnitudes are $M_{0}+\Delta$ or greater and picks associated foreshocks and aftershocks only using events $\pm \Delta$ magnitude units removed from the mainshock. Significant differences in the clustered and background mixing proportions between the traditional and $\Delta$-analysis may indicate that the 
detection of clustered events is being affected by the chosen cut-off. Conducting a $\Delta$-analysis as a supplementary test of sampling uncertainty would be beneficial for the present study, in addition to the relatively straightforward sensitivity analyses provided in the appendices.

A possible next step for the identification and assessment of the spatiotemporally varying rates of seismicity within the WCSB would be the regional application of a space-time ETAS model (e.g. Harte, 2014; Jalilian, 2019; Ogata \& Zhuang, 2006). The ETAS model used in Chapter 4's cluster analyses is dependent only on event magnitude and time of occurrence; its parameters are determined independent of event locations. It is therefore more applicable to a specific aftershock sequence or a cluster of events where relative event locations are less important. The parameters of a space-time ETAS model, on the other hand, are spatially dependent and hence much more capable of characterizing seismicity from a regional catalogue. The results of the space-time model could then be compared with the NND results to identify possible correlations between parameter values (particularly the magnitude weighting factor $\alpha$ ) and areas of heightened loosely and/or tightly clustered activity. Another application of the spacetime ETAS model would be on a dataset such as the Fox Creek cluster, which contained numerous, spatiotemporally distinct seismic sequences. The analysis conducted here optimized only one set of ETAS parameters for all the individual sequences, whereas the space-time model would be capable of showing parametric variation between the sequences.

\subsection{Final Remarks}

The results of this study add to the ongoing scientific discussion regarding induced seismicity and its implications for seismic hazard assessment. Within the WCSB, statistical differences were found occurring between natural and induced seismicity; specifically that induced activity transpired as both a measurably elevated (loosely clustered) "background" rate and as tightly clustered event sequences, whereas the natural activity was largely reminiscent of a Poisson point process. The identification of these separable earthquake subpopulations improves the understanding of induced earthquake processes and may help in the detection of unnatural activity in other regions, possibly by isolating areas with comparably lower inter-event distances. Moreover, distinctive statistical properties of induced clustering triggered by specific anthropogenic endeavors were identified, including the tendency of hydraulic fracturing 
operations to result in significantly higher levels of tightly clustered, swarm-like behavior than either conventional fuel extraction or wastewater disposal. The propensity of HF operations to stimulate swarm-like activity is important information for energy industry personnel to know, as it may help for the more rapid identification and prediction of future induced earthquakes. 


\section{References}

Abercrombie, R. (2006). Earthquakes: Radiated Energy and the Physics of Faulting (Vol. 170). Washington, DC: American Geophysical Union.

Abercrombie, R. E., \& Brune, J. N. (1994). Evidence for a constantb-value above magnitude 0 in the southern San Andreas, San Jacinto and San Miguel Fault Zones, and at the Long Valley Caldera, California. Geophysical Research Letters, 21(15), 1647-1650. https://doi.org/10.1029/94GL01138

Adams, J., \& Halchuk, S. (2003). Fourth Generation Seismic Hazard Maps of Canada: Values for Over 650 Canadian Localities Intended for the 2005 National Building Code of Canada. https://doi.org/10.4095/214223

Ader, T. J., \& Avouac, J.-P. (2013). Detecting periodicities and declustering in earthquake catalogs using the Schuster spectrum, application to Himalayan seismicity. Earth and Planetary Science Letters, 377-378(Journal Article), 97-105. https://doi.org/10.1016/j.epsl.2013.06.032

Akaike, H. (1974). A new look at the statistical model identification. IEEE Transactions on Automatic Control, 19(6), 716-723. https://doi.org/10.1109/TAC.1974.1100705

Aki, K. (1965). Maximum Likelihood Estimate of $b$ in the Formula $\log \mathrm{N}=\mathrm{a}-\mathrm{bM}$ and its Confidence Limits. Bull. Earthq. Res. Inst. Tokyo Univ, 43, 237-239.

Atkinson, G. M. (2017). Strategies to prevent damage to critical infrastructure due to induced seismicity. FACETS, 2(1), 374-394. https://doi.org/10.1139/facets-2017-0013

Atkinson, G. M., Eaton, D. W., Ghofrani, H., Walker, D., Cheadle, B., Schultz, R., ... Kao, H. (2016). Hydraulic Fracturing and Seismicity in the Western Canada Sedimentary Basin. Seismological Research Letters, 87(3), 631-647. https://doi.org/10.1785/0220150263

Atkinson, G. M., Greig, D. W., \& Yenier, E. (2014). Estimation of Moment Magnitude (M) for Small Events $(\mathrm{M}<4)$ on Local Networks. Seismological Research Letters, 85(5), 11161124. https://doi.org/10.1785/0220130180

Bachmann, C. E., Wiemer, S., Goertz-Allmann, B. P., \& Woessner, J. (2012). Influence of pore-pressure on the event-size distribution of induced earthquakes. Geophysical Research Letters, 39(9). https://doi.org/10.1029/2012GL051480 
Baiesi, M., \& Paczuski, M. (2004). Scale-free networks of earthquakes and aftershocks. Physical Review. E, Statistical, Nonlinear, and Soft Matter Physics, 69(6). https://doi.org/10.1103/PhysRevE.69.066106

Bao, X., \& Eaton, D. W. (2016). Fault activation by hydraulic fracturing in western Canada. Science (New York, N.Y.), 354(6318), 1406-1409. https://doi.org/10.1126/science.aag2583

Baranova, V., Mustaqeem, A., \& Bell, S. (1999). A model for induced seismicity caused by hydrocarbon production in the Western Canada Sedimentary Basin. Canadian Journal of Earth Sciences, 36(1), 47-64. https://doi.org/10.1139/cjes-36-1-47

B.C. Oil and Gas Commission. (2012). Investigation of observed seismicity in the Horn River Basin. Victoria, B.C.: BC Oil and Gas Commission.

B.C. Oil and Gas Commission. (2014). Investigation of observed seismicity in the Montney Trend. Victoria, B.C.: BC Oil and Gas Commission.

Bender, B. (1983). Maximum likelihood estimation of $b$ values for magnitude grouped data. Bulletin of the Seismological Society of America, 73(3), 831.

Ben-Zion, Y., \& Lyakhovsky, V. (2006). Analysis of aftershocks in a lithospheric model with seismogenic zone governed by damage rheology. Geophysical Journal International, 165(1), 197-210. https://doi.org/10.1111/j.1365-246X.2006.02878.x

Bhattacharya, P., Phan, M., \& Shcherbakov, R. (2011). Statistical Analysis of the 2002 Mw 7.9 Denali Earthquake Aftershock SequenceStatistical Analysis of the 2002 Mw 7.9 Denali Earthquake Aftershock Sequence. Bulletin of the Seismological Society of America, 101(6), 2662-2674. https://doi.org/10.1785/0120100336

Bhattacharya, P., \& Viesca, R. C. (2019). Fluid-induced aseismic fault slip outpaces pore-fluid migration. Science (New York, N.Y.), 364(6439), 464-468. https://doi.org/10.1126/science.aaw7354

Bormann, P., \& Dewey, J. W. (2012). The new IASPEI standards for determining magnitudes from digital data and their relation to classical magnitudes. In New Manual of Seismological Observatory Practice 2 (NMSOP-2) (pp. 1-44). https://doi.org/10.2312/GFZ.NMSOP-2_IS_3.3 
Boyd, O. S. (2012). Including Foreshocks and Aftershocks in Time-Independent Probabilistic Seismic-Hazard Analyses. Bulletin of the Seismological Society of America, 102(3), 909-917. https://doi.org/10.1785/0120110008

Bridges, D. L., \& Gao, S. S. (2006). Spatial variation of seismic b-values beneath Makushin Volcano, Unalaska Island, Alaska. Earth and Planetary Science Letters, 245(1), 408415. https://doi.org/10.1016/j.eps1.2006.03.010

Brodsky, E. E., \& Lajoie, L. J. (2013). Anthropogenic Seismicity Rates and Operational Parameters at the Salton Sea Geothermal Field. Science, 341(6145), 543-546. https://doi.org/10.1126/science.1239213

Brudzinski, M. R., \& Kozłowska, M. (2019). Seismicity induced by hydraulic fracturing and wastewater disposal in the Appalachian Basin, USA: a review. Acta Geophysica, 67(1), 351-364. https://doi.org/10.1007/s11600-019-00249-7

Cao, A., \& Gao, S. S. (2002). Temporal variation of seismic b-values beneath northeastern Japan island arc. Geophysical Research Letters, 29(9). https://doi.org/10.1029/2001GL013775

Chan, C.-H., Wu, Y.-M., Tseng, T.-L., Lin, T.-L., \& Chen, C.-C. (2012). Spatial and temporal evolution of b-values before large earthquakes in Taiwan. Tectonophysics, 532535(Journal Article), 215-222. https://doi.org/10.1016/j.tecto.2012.02.004

Chang, K. W., \& Segall, P. (2016). Injection-induced seismicity on basement faults including poroelastic stressing. Journal of Geophysical Research: Solid Earth, 121(4), 27082726. https://doi.org/10.1002/2015JB012561

Chen, S. (2010). Earthquake Source Scaling: M1 versus Other Magnitude Scales. Bulletin of the Seismological Society of America, 100(2), 859-867. https://doi.org/10.1785/0120090167

Chen, S., \& Atkinson, G. M. (2002). Global Comparisons of Earthquake Source Spectra. Bulletin of the Seismological Society of America, 92(3), 885-895. https://doi.org/10.1785/0120010152

Clerc, F., Harrington, R. M., Liu, Y., \& Gu, Y. J. (2016). Stress drop estimates and hypocenter relocations of induced seismicity near Crooked Lake, Alberta. Geophysical Research Letters, 43(13), 6942-6951. https://doi.org/10.1002/2016GL069800 
Console, R., Jackson, D. D., \& Kagan, Y. Y. (2010). Using the ETAS Model for Catalog Declustering and Seismic Background Assessment. Pure and Applied Geophysics, 167(6), 819-830. https://doi.org/10.1007/s00024-010-0065-5

Cornell, C. A. (1968). Engineering seismic risk analysis. Bulletin of the Seismological Society of America, 58(5), 1583.

Cui, L., \& Atkinson, G. M. (2016). Spatiotemporal Variations in the Completeness Magnitude of the Composite Alberta Seismicity Catalog (CASC). Seismological Research Letters, 87(4), 853-863. https://doi.org/10.1785/0220150268

Cui, L., Fereidoni, A., \& Atkinson, G. (2015). Compilation of Composite Alberta Seismicity Catalog (CASC) for Earthquake Hazard from Induced Seismicity in Alberta.

Deng, K., Liu, Y., \& Harrington, R. M. (2016). Poroelastic stress triggering of the December 2013 Crooked Lake, Alberta, induced seismicity sequence: POROELASTIC STRESSING-INDUCED SEISMICITY. Geophysical Research Letters, 43(16), 84828491. https://doi.org/10.1002/2016GL070421

Doglioni, C. (2018). A classification of induced seismicity. Geoscience Frontiers, 9(6), 19031909. https://doi.org/10.1016/j.gsf.2017.11.015

Eaton, D. W. (2018). Passive seismic monitoring of induced seismicity: Fundamental principles and application to energy technologies. Cambridge University Press.

Eaton, D. W., Igonin, N., Poulin, A., Weir, R., Zhang, H., Pellegrino, S., \& Rodriguez, G. (2018). Induced Seismicity Characterization during Hydraulic-Fracture Monitoring with a Shallow-Wellbore Geophone Array and Broadband Sensors. Seismological Research Letters, 89(5), 1641-1651. https://doi.org/10.1785/0220180055

Eaton, D. W., \& Mahani, A. B. (2015). Focal Mechanisms of Some Inferred Induced Earthquakes in Alberta, Canada. Seismological Research Letters, 86(4), 1078-1085. https://doi.org/10.1785/0220150066

El-Isa, Z. H., \& Eaton, D. W. (2014). Spatiotemporal variations in the b-value of earthquake magnitude-frequency distributions: Classification and causes. Tectonophysics, $615-$ 616(Journal Article), 1-11. https://doi.org/10.1016/j.tecto.2013.12.001

Ellsworth, W. L. (2013). Injection-Induced Earthquakes. Science, 341(6142), 142-142. https://doi.org/10.1126/science.1225942 
Farrell, J., Husen, S., \& Smith, R. B. (2009). Earthquake swarm and b-value characterization of the Yellowstone volcano-tectonic system. Journal of Volcanology and Geothermal Research, 188(1), 260-276. https://doi.org/10.1016/j.jvolgeores.2009.08.008

Felzer, K. (2006). Calculating the Gutenberg-Richter b value. AGU Fall Meeting Abstracts, 08.

Fereidoni, A., Atkinson, G. M., Macias, M., \& Goda, K. (2012). CCSC: A Composite Seismicity Catalog for Earthquake Hazard Assessment in Major Canadian Cities. Seismological Research Letters, 83(1), 179-189. https://doi.org/10.1785/gssrl.83.1.179

Galloway, E., Hauck, T., Corlett, H., Pană, D., \& Schultz, R. (2018). Faults and associated karst collapse suggest conduits for fluid flow that influence hydraulic fracturing-induced seismicity. Proceedings of the National Academy of Sciences of the United States of America, 115(43), E10003-E10012. https://doi.org/10.1073/pnas.1807549115

Gardner, J. K., \& Knopoff, L. (1974). Is the sequence of earthquakes in Southern California, with aftershocks removed, Poissonian? Bulletin of the Seismological Society of America, 64(5), 1363-1367.

Ghofrani, H., \& Atkinson, G. M. (2016). A preliminary statistical model for hydraulic fractureinduced seismicity in the Western Canada Sedimentary Basin. Geophysical Research Letters, 43(19), 172. https://doi.org/10.1002/2016GL070042

Godano, C. (2017). A new method for the estimation of the completeness magnitude. Physics of the Earth and Planetary Interiors, 263(Journal Article), 7-11. https://doi.org/10.1016/j.pepi.2016.12.003

Goebel, T. H. W., Hauksson, E., Aminzadeh, F., \& Ampuero, J.-P. (2015). An objective method for the assessment of fluid injection-induced seismicity and application to tectonically active regions in central California. Journal of Geophysical Research: Solid Earth, 120(10), 7013-7032. https://doi.org/10.1002/2015JB011895

Goertz-Allmann, B. P., \& Wiemer, S. (2013). Geomechanical modeling of induced seismicity source parameters and implications for seismic hazard assessment. Geophysics, 78(1), 25-39. https://doi.org/10.1190/geo2012-0102.1

Gomberg, J. (1991). Seismicity and detection/location threshold in the Southern Great Basin Seismic Network. Journal of Geophysical Research, 96(B10), 16401. https://doi.org/10.1029/91JB01593 
Grigoli, F., Cesca, S., Priolo, E., Rinaldi, A. P., Clinton, J. F., Stabile, T. A., ... Lawrence Berkeley National Lab. (LBNL), B., CA (United States). (2017). Current challenges in monitoring, discrimination, and management of induced seismicity related to underground industrial activities: A European perspective. Reviews of Geophysics, 55(2), 310-340. https://doi.org/10.1002/2016RG000542

Guest, A., Rebel, E., Bailly, S. M., \& Kostadinovic, J. (2014). Integrated analysis for fracture identification during hydraulic fracturing. Presented at the SPE Annual Technical Conference and Exhibition, Society of Petroleum Engineers.

Gutenberg, B., \& Richter, C. F. (1944). Frequency of earthquakes in California*. Bulletin of the Seismological Society of America, 34(4), 185-188.

Hainzl, S. (2004). Seismicity patterns of earthquake swarms due to fluid intrusion and stress triggering. Geophysical Journal International, 159(3), 1090-1096. https://doi.org/10.1111/j.1365-246X.2004.02463.x

Hainzl, S., \& Ogata, Y. (2005). Detecting fluid signals in seismicity data through statistical earthquake modeling. Journal of Geophysical Research - Solid Earth, 110(B5), B05S07. https://doi.org/10.1029/2004JB003247

Hajati, T., Langenbruch, C., \& Shapiro, S. A. (2015). A statistical model for seismic hazard assessment of hydraulic-fracturing-induced seismicity. Geophysical Research Letters, 42(24), 10,601-10,606. https://doi.org/10.1002/2015GL066652

Hamiel, Y., Lyakhovsky, V., \& Agnon, A. (2004). Coupled evolution of damage and porosity in poroelastic media: Theory and applications to deformation of porous rocks. Geophysical Journal International, 156(3), 701-713. https://doi.org/10.1111/j.1365246X.2004.02172.X

Hanks, T. C., \& Kanamori, H. (1979). Fault mechanics. Journal of Geophysical Research, 84(B5), 2145. https://doi.org/10.1029/JB084iB05p02145

Harte, D. S. (2014). An ETAS model with varying productivity rates. Geophysical Journal International, 198(1), 270-284. https://doi.org/10.1093/gji/ggu129

Harvey, D., \& Hansen, R. (1994). Contributions of IRIS data to nuclear monitering. Incorporated Research Institutions for Seismology (IRIS) Newsletter, 13(2). 
Hastie, T., Tibshirani, R., \& Friedman, J. H. (2009). The EM Algorithm. In The elements of statistical learning: Data mining, inference, and prediction (2nd ed., pp. 236-243). New York: Springer.

Hicks, A. L. (2011). Clustering in multidimensional spaces with applications to statistical analysis of earthquake clustering. ProQuest Dissertations Publishing.

Hill, D. P., Ellsworth, W. L., Johnston, M. J. S., Langbein, J. O., Oppenheimer, D. H., PITT, A. M., ... MCNUTT, S. R. (1990). The 1989 earthquake swarm beneath Mammoth Mountain, California: An initial look at the 4 May through 30 September activity. Bulletin of the Seismological Society of America, 80(2), 325.

Holland, A. A. (2013). Earthquakes Triggered by Hydraulic Fracturing in South-Central Oklahoma. Bulletin of the Seismological Society of America, 103(3), 1784-1792. https://doi.org/10.1785/0120120109

Hornbach, M. J., Jones, M., Scales, M., DeShon, H. R., Magnani, M. B., Frohlich, C., ... Layton, M. (2016). Ellenburger wastewater injection and seismicity in North Texas. Physics of the Earth and Planetary Interiors, 261(Journal Article), 54-68. https://doi.org/10.1016/j.pepi.2016.06.012

Horner, R. B., Barclay, J. E., \& MacRae, J. M. (1994). Earthquakes and Hydrocarbon Production in the Fort St. John Area of Northeastern British Columbia. Canadian Journal of Exploration Geophysics, 30(1), 38-50.

Horton, S. (2012). Disposal of Hydrofracking Waste Fluid by Injection into Subsurface Aquifers Triggers Earthquake Swarm in Central Arkansas with Potential for Damaging Earthquake. Seismological Research Letters, 83(2), 250-260. https://doi.org/10.1785/gssrl.83.2.250

Huang, Y., \& Beroza, G. C. (2015). Temporal variation in the magnitude-frequency distribution during the Guy-Greenbrier earthquake sequence. Geophysical Research Letters, 42(16), 6639-6646. https://doi.org/10.1002/2015GL065170

Huang, Y., Shi-Yong, Z., \& Jian-Cang, Z. (2016). Numerical Tests on Catalog-Based Methods to Estimate Magnitude of Completeness. Chinese Journal of Geophysics, 59(3), 266275. https://doi.org/10.1002/cjg2.20232

Husen, S., Taylor, R., Smith, R. B., \& Healser, H. (2004). Changes in geyser eruption behavior and remotely triggered seismicity in Yellowstone National Park produced by the 2002 
M 7.9 Denali fault earthquake, Alaska. Geology, 32(6), 537.

https://doi.org/10.1130/G20381.1

Hyder, J. P., \& Lerner, A. W. (2014). Hydraulic Fracturing. In K. L. Lerner \& B. W. Lerner (Eds.), The Gale Encyclopedia of Science (5th ed., Vol. 4, pp. 2250-2252). Farmington Hills, MI: Gale.

Igonin, N., Zecevic, M., \& Eaton, D. W. (2018). Bilinear Magnitude-Frequency Distributions and Characteristic Earthquakes During Hydraulic Fracturing. Geophysical Research Letters, 45(23), 12,866-12,874. https://doi.org/10.1029/2018GL079746

Imoto, M. (1991). Changes in the magnitude-Frequency b-value prior to large $(M \geqslant 6.0)$ earthquakes in Japan. Tectonophysics, 193(4), 311-325. https://doi.org/10.1016/00401951(91)90340-X

Iwata, T. (2008). Low detection capability of global earthquakes after the occurrence of large earthquakes: Investigation of the Harvard CMT catalogue. Geophysical Journal International, 174(3), 849-856. https://doi.org/10.1111/j.1365-246X.2008.03864.x

Jalilian, A. (2019). ETAS: An R package for fitting the space-time ETAS model to earthquake data. Journal of Statistical Software, 88(1), 1-39. https://doi.org/10.18637/jss.v088.c01

Jordan, T. H., McGuire, J. J., \& Boettcher, M. S. (2005). Foreshock sequences and short-term earthquake predictability on East Pacific Rise transform faults. Nature, 434(7032), 457461. https://doi.org/10.1038/nature03377

Kagan, Y. Y. (1991). Fractal dimension of brittle fracture. Journal of Nonlinear Science, 1(1), 1-16. https://doi.org/10.1007/BF01209146

Kanamori, H., \& Brodsky, E. E. (2001). The Physics of Earthquakes. Physics Today, 54(6), 34 40. https://doi.org/10.1063/1.1387590

Kao, H., Hyndman, R., Jiang, Y., Visser, R., Smith, B., Babaie Mahani, A., ... He, J. (2018). Induced Seismicity in Western Canada Linked to Tectonic Strain Rate: Implications for Regional Seismic Hazard. Geophysical Research Letters, 45(20), 11,104-11,115. https://doi.org/10.1029/2018GL079288

Keranen, K. M., \& Weingarten, M. (2018). Induced Seismicity. Annual Review of Earth and Planetary Sciences, 46(1), 149-174. https://doi.org/10.1146/annurev-earth-082517010054 
Keranen, K. M., Weingarten, M., Abers, G. A., Bekins, B. A., \& Ge, S. (2014). Sharp increase in central Oklahoma seismicity since 2008 induced by massive wastewater injection. Science, 345(6195), 448-451. https://doi.org/10.1126/science.1255802

Kettlety, T., Verdon, J. P., Werner, M. J., Kendall, J. M., \& Budge, J. (2019). Investigating the role of elastostatic stress transfer during hydraulic fracturing-induced fault activation. Geophysical Journal International, (Journal Article). https://doi.org/10.1093/gji/ggz080

Kilb, D., Gomberg, J., \& Bodin, P. (2000). Triggering of earthquake aftershocks by dynamic stresses. Nature, 408(6812), 570-574. https://doi.org/10.1038/35046046

King, G. E. (2010). Thirty Years of Gas Shale Fracturing: What Have We Learned? In SPE133456-MS (p. 50). SPE: Society of Petroleum Engineers. https://doi.org/10.2118/133456-MS

Kosobokov, V., \& Mazhkenov, S. A. (2013). On Similarity in the Spatial Distribution of Seismicity. In Computational Seismology and Geodynamics (Vol. 22). https://doi.org/10.1029/CS001p0006

Kozłowska, M., Brudzinski, M. R., Friberg, P., Skoumal, R. J., Baxter, N. D., \& Currie, B. S. (2018). Maturity of nearby faults influences seismic hazard from hydraulic fracturing. Proceedings of the National Academy of Sciences of the United States of America, 115(8), E1720-E1729. https://doi.org/10.1073/pnas.1715284115

Kumazawa, T., \& Ogata, Y. (2013). Quantitative description of induced seismic activity before and after the 2011 Tohoku-Oki earthquake by nonstationary ETAS models. Journal of Geophysical Research: Solid Earth, 118(12), 6165-6182. https://doi.org/10.1002/2013JB010259

Kwiatek, G., Plenkers, K., \& Dresen, G. (2011). Source Parameters of Picoseismicity Recorded at Mponeng Deep Gold Mine, South Africa: Implications for Scaling Relations. Bulletin of the Seismological Society of America, 101(6), 2592-2608. https://doi.org/10.1785/0120110094

Langenbruch, C., \& Shapiro, S. A. (2010). Decay rate of fluid-induced seismicity after termination of reservoir stimulations. Geophysics, 75(6), MA53-MA62. https://doi.org/10.1190/1.3506005 
Langevin, C. D., Hughes, J. D., Banta, E. R., Niswonger, R. G., Panday, S., \& Provost, A. M. (2017). Documentation for the MODFLOW 6 Groundwater Flow Model (Report No. 6A55). Reston, VA. https://doi.org/10.3133/tm6A55

Lei, X., Yu, G., Ma, S., Wen, X., \& Wang, Q. (2008). Earthquakes induced by water injection at $\sim 3 \mathrm{~km}$ depth within the Rongchang gas field, Chongqing, China. Journal of Geophysical Research - Solid Earth, 113(B10), B10310. https://doi.org/10.1029/2008JB005604

Llenos, A. L., \& Michael, A. J. (2013). Modeling Earthquake Rate Changes in Oklahoma and Arkansas: Possible Signatures of Induced Seismicity. Bulletin of the Seismological Society of America, 103(5), 2850-2861. https://doi.org/10.1785/0120130017

Lombardi, A. M., Cocco, M., \& Marzocchi, W. (2010). On the Increase of Background Seismicity Rate during the 1997-1998 Umbria-Marche, Central Italy, Sequence: Apparent Variation or Fluid-Driven Triggering? Bulletin of the Seismological Society of America, 100(3), 1138-1152. https://doi.org/10.1785/0120090077

Maghsoudi, S., Baró, J., Kent, A., Eaton, D., \& Davidsen, J. (2018). Interevent Triggering in Microseismicity Induced by Hydraulic Fracturing. Bulletin of the Seismological Society of America, 108(3A), 1133-1146. https://doi.org/10.1785/0120170368

Maghsoudi, S., Eaton, D. W., \& Davidsen, J. (2016). Nontrivial clustering of microseismicity induced by hydraulic fracturing. Geophysical Research Letters, 43(20), 10-10,679. https://doi.org/10.1002/2016GL070983

Martínez-Garzón, P., Zaliapin, I., Ben-Zion, Y., Kwiatek, G., \& Bohnhoff, M. (2018). Comparative Study of Earthquake Clustering in Relation to Hydraulic Activities at Geothermal Fields in California. Journal of Geophysical Research: Solid Earth, 123(5), 4041-4062. https://doi.org/10.1029/2017JB014972

McClure, M., Gibson, R., Chiu, K.-K., \& Ranganath, R. (2017). Identifying potentially induced seismicity and assessing statistical significance in Oklahoma and California: Induced Seismicity Statistics. Journal of Geophysical Research: Solid Earth, (Journal Article). https://doi.org/10.1002/2016JB013711

McGarr, A., \& Barbour, A. J. (2017). Wastewater Disposal and the Earthquake Sequences During 2016 Near Fairview, Pawnee, and Cushing, Oklahoma: Induced Earthquake 
Sequences During 2016. Geophysical Research Letters, 44(18), 9330-9336.

https://doi.org/10.1002/2017GL075258

McGuire, R. K. (2004). Seismic hazard and risk analysis (Vol. MNO-10). Oakland, Calif: Earthquake Engineering Research Institute.

Mignan, A. (2011). Retrospective on the Accelerating Seismic Release (ASR) hypothesis: Controversy and new horizons. Tectonophysics, 505(1), 1-16. https://doi.org/10.1016/j.tecto.2011.03.010

Mignan, A. (2012). Functional shape of the earthquake frequency-magnitude distribution and completeness magnitude: FUNCTIONAL SHAPE OF THE FMD. Journal of Geophysical Research: Solid Earth, 117(B8), n/a-n/a. https://doi.org/10.1029/2012JB009347

Mignan, A., \& Chouliaras, G. (2014). Fifty Years of Seismic Network Performance in Greece (1964-2013): Spatiotemporal Evolution of the Completeness Magnitude. Seismological Research Letters, 85(3), 657-667. https://doi.org/10.1785/0220130209

Mignan, A., Danciu, L., \& Giardini, D. (2018). Considering large earthquake clustering in seismic risk analysis. Natural Hazards, 91(S1), 149-172. https://doi.org/10.1007/s11069-016-2549-9

Mignan, A., Werner, M. J., Wiemer, S., Chen, C., \& Wu, Y. (2011). Bayesian Estimation of the Spatially Varying Completeness Magnitude of Earthquake Catalogs. Bulletin of the Seismological Society of America, 101(3), 1371-1385. https://doi.org/10.1785/0120100223

Moczo, P., Kristek, J., \& Gális, M. (2014). The finite-difference modelling of earthquake motions: Waves and ruptures. New York: Cambridge University Press.

Mogi, K. (1963). Some Discussions on Aftershocks, Foreshocks and Earthquake Swarms: The Fracture of a Semi-infinite Body Caused by an Inner Stress Origin and Its Relation to the Earthquake Phenomena (Third Paper). Bull. Earthq. Res. Inst. Tokyo Univ, 41, 615658.

Montuori, C., Falcone, G., Murru, M., Thurber, C., Reyners, M., \& Eberhart-Phillips, D. (2010). Crustal heterogeneity highlighted by spatial b-value map in the Wellington region of New Zealand: B-value map in the Wellington region. Geophysical Journal International, 183(1), 451-460. https://doi.org/10.1111/j.1365-246X.2010.04750.x 
Morita, Y., Nakao, S., \& Hayashi, Y. (2006). A quantitative approach to the dike intrusion process inferred from a joint analysis of geodetic and seismological data for the 1998 earthquake swarm off the east coast of Izu Peninsula, central Japan. Journal of Geophysical Research: Solid Earth, 111(B6). https://doi.org/10.1029/2005JB003860

Nanjo, K. Z., Enescu, B., Shcherbakov, R., Turcotte, D. L., Iwata, T., \& Ogata, Y. (2007). Decay of aftershock activity for Japanese earthquakes. Journal of Geophysical Research - Solid Earth, 112(B8), B08309. https://doi.org/10.1029/2006JB004754

Nava, F. A., Ávila-Barrientos, L., Márquez-Ramírez, V. H., Torres, I., \& Zúñiga, F. R. (2018). Sampling uncertainties and source b likelihood for the Gutenberg-Richter b value from the Aki-Utsu method. Journal of Seismology, 22(1), 315-324. https://doi.org/10.1007/s10950-017-9707-8

Nava, F. A., Márquez-Ramírez, V. H., Zúñiga, F. R., Ávila-Barrientos, L., \& Quinteros, C. B. (2017a). Gutenberg-Richter b-value maximum likelihood estimation and sample size. Journal of Seismology, 21(1), 127-135. https://doi.org/10.1007/s10950-016-9589-1

Nava, F. A., Márquez-Ramírez, V. H., Zúñiga, F. R., \& Lomnitz, C. (2017b). GutenbergRichter b-value determination and large-magnitudes sampling. Journal of Seismology, 87(1), 1-11. https://doi.org/10.1007/s11069-017-2750-5

Nuannin, P., Kulhánek, O., Persson, L., Teknisk-naturvetenskapliga vetenskapsområdet, Uppsala universitet, Geovetenskapliga sektionen, ... Geofysik. (2012). Variations of bvalues preceding large earthquakes in the Andaman-Sumatra subduction zone. Journal of Asian Earth Sciences, 61(SI), 237-242. https://doi.org/10.1016/j.jseaes.2012.10.013

Ogata, Y. (1988). Statistical Models for Earthquake Occurrences and Residual Analysis for Point Processes. Journal of the American Statistical Association, 83(401), 9-27. https://doi.org/10.1080/01621459.1988.10478560

Ogata, Y. (1989). Statistical model for standard seismicity and detection of anomalies by residual analysis. Tectonophysics, 169(1), 159-174. https://doi.org/10.1016/00401951(89)90191-1

Ogata, Y. (1992). Detection of precursory relative quiescence before great earthquakes through a statistical model. Journal of Geophysical Research, 97(B13), 19845. https://doi.org/10.1029/92JB00708 
Ogata, Y., \& Shimazaki, K. (1984). Transition from aftershock to normal activity: The 1965 Rat Islands earthquake aftershock sequence. Bulletin of the Seismological Society of America, 74(5), 1757.

Ogata, Y., Utsu, T., \& Katsura, K. (1996). Statistical discrimination of foreshocks from other earthquake clusters. Geophysical Journal International, 127(1), 17-30. https://doi.org/10.1111/j.1365-246X.1996.tb01531.x

Ogata, Y., \& Zhuang, J. (2006). Space-time ETAS models and an improved extension. Tectonophysics, 413(1), 13-23. https://doi.org/10.1016/j.tecto.2005.10.016

Ogwari, P. O., \& Horton, S. P. (2016). Numerical model of pore-pressure diffusion associated with the initiation of the 2010-2011 Guy-Greenbrier, Arkansas earthquakes. Geofluids, 16(5), 954-970. https://doi.org/10.1111/gfl.12198

Omi, T., Ogata, Y., Hirata, Y., \& Aihara, K. (2014). Estimating the ETAS model from an early aftershock sequence. Geophysical Research Letters, 41(3), 850-857. https://doi.org/10.1002/2013GL058958

Omori, F. (1894). On the aftershocks of earthquakes. Journal of the College of Science, Imperial University of Tokyo, (7), 111-200.

Ordaz, M., \& Faccioli, E. (2018). Modelling correlation between Gutenberg-Richter parameters $\mathrm{a}$ and $\mathrm{b}$ in PSHA. Bulletin of Earthquake Engineering, 16(5), 1829-1846. https://doi.org/10.1007/s10518-017-0274-8

Pawley, S., Schultz, R., Playter, T., Corlett, H., Shipman, T., Lyster, S., \& Hauck, T. (2018). The Geological Susceptibility of Induced Earthquakes in the Duvernay Play. Geophysical Research Letters, 45(4), 1786-1793. https://doi.org/10.1002/2017GL076100

Petersen, M. D., Mueller, C. S., Moschetti, M. P., Hoover, S. M., Llenos, A. L., Ellsworth, W. L., ... Rukstales, K. S. (2016). Seismic-Hazard Forecast for 2016 Including Induced and Natural Earthquakes in the Central and Eastern United States. Seismological Research Letters, 87(6), 1327-1341. https://doi.org/10.1785/0220160072

Prasad, S., \& Singh, C. (2015). Evolution of b-values before large earthquakes of magnitude greater than or equal to 6.0 in the Andaman region. Geologica Acta, 13(3), 205. https://doi.org/10.1344/GeologicaActa2015.13.3.3 
Reasenberg, P. (1985). Second-order moment of central California seismicity, 1969-1982. Journal of Geophysical Research: Solid Earth, 90(B7), 5479-5495. https://doi.org/10.1029/JB090iB07p05479

Richter, C. F., \& Gutenberg, B. (1956). Magnitude and Energy of Earthquakes. Annals of Geophysics, 9(1), 1-15. https://doi.org/10.4401/ag-5590

Rogers, G. C., \& Horner, R. B. (1991). An Overview of Western Canadian Seismicity. In D. B. Slemmons, E. R. Engdahl, M. D. Zoback, \& D. D. Blackwell (Eds.), Neotectonics of North America (Vol. 1, pp. 69-76). Geological Society of America.

Rutledge, J. T., Phillips, W. S., \& Mayerhofer, M. J. (2004). Faulting Induced by Forced Fluid Injection and Fluid Flow Forced by Faulting: An Interpretation of Hydraulic-Fracture Microseismicity, Carthage Cotton Valley Gas Field, Texas. Bulletin of the Seismological Society of America, 94(5), 1817-1830. https://doi.org/10.1785/012003257

Rydelek, P. A., \& Sacks, I. S. (1989). Testing the completeness of earthquake catalogues and the hypothesis of self-similarity. Nature, 337(6204), 251-253. https://doi.org/10.1038/337251a0

Sadovsky, M. A., Golubeva, T. V., Pisarenko, V. F., \& Shninman, M. G. (1984). Characteristic dimension of rocks and hierarchical properties of seismicity. Izy. Acad. Sci. USSR. Phys. Solid Earth, 20, 87-95.

Sagiya, T., Stein, R. S., \& Toda, S. (2002). Evidence from the ad 2000 Izu islands earthquake swarm that stressing rate governs seismicity. Nature, 419(6902), 58-61. https://doi.org/10.1038/nature00997

Sánchez C, E., \& Vega-Jorquera, P. (2018). New Bayesian frequency-magnitude distribution model for earthquakes applied in Chile. Physica A: Statistical Mechanics and Its Applications, 508(Journal Article), 305-312. https://doi.org/10.1016/j.physa.2018.05.119

Sandri, L., \& Marzocchi, W. (2003). A review and new insights on the estimation of the bvalueand its uncertainty. Annals of Geophysics, 46(6). https://doi.org/10.4401/ag-3472

Schaefer, A. M., Daniell, J. E., \& Wenzel, F. (2017). The smart cluster method: Adaptive earthquake cluster identification and analysis in strong seismic regions. Journal of Seismology, 21(4), 965-985. https://doi.org/10.1007/s10950-017-9646-4 
Schoenball, M., Davatzes, N. C., \& Glen, J. M. G. (2015). Differentiating induced and natural seismicity using space-time-magnitude statistics applied to the Coso Geothermal field. Geophysical Research Letters, 42(15), 6221-6228. https://doi.org/10.1002/2015GL064772

Schoenball, M., \& Ellsworth, W. L. (2017). A Systematic Assessment of the Spatiotemporal Evolution of Fault Activation Through Induced Seismicity in Oklahoma and Southern Kansas. Journal of Geophysical Research: Solid Earth, 122(12), 10,189-10,206. https://doi.org/10.1002/2017JB014850

Scholz, C. H. (2002). The mechanics of earthquakes and faulting (2nd ed.). New York;Cambridge, U.K; Cambridge University Press.

Schorlemmer, D., Wiemer, S., \& Wyss, M. (2004a). Earthquake statistics at Parkfield: 1. Stationarity of b values. Journal of Geophysical Research - Solid Earth, 109(B12), B12307. https://doi.org/10.1029/2004JB003234

Schorlemmer, D., Wiemer, S., Wyss, M., \& Jackson, D. D. (2004b). Earthquake statistics at Parkfield: 2. Probabilistic forecasting and testing. Journal of Geophysical Research Solid Earth, 109(B12), B12308. https://doi.org/10.1029/2004JB003235

Schultz, R., Atkinson, G., Eaton, D. W., Gu, Y. J., \& Kao, H. (2018). Hydraulic fracturing volume is associated with induced earthquake productivity in the Duvernay play. Science, 359(6373), 304-308. https://doi.org/10.1126/science.aao0159

Schultz, R., Corlett, H., Haug, K., Kocon, K., MacCormack, K., Stern, V., \& Shipman, T. (2016). Linking fossil reefs with earthquakes: Geologic insight to where induced seismicity occurs in Alberta. Geophysical Research Letters, 43(6), 2534-2542. https://doi.org/10.1002/2015GL067514

Schultz, R., Mei, S., Pană, D., Stern, V., Gu, Y. J., Kim, A., \& Eaton, D. (2015a). The Cardston Earthquake Swarm and Hydraulic Fracturing of the Exshaw Formation (Alberta Bakken Play). Bulletin of the Seismological Society of America, 105(6), 2871-2884. https://doi.org/10.1785/0120150131

Schultz, R., Stern, V., \& Gu, Y. J. (2014). An investigation of seismicity clustered near the Cordel Field, west central Alberta, and its relation to a nearby disposal well. Journal of Geophysical Research: Solid Earth, 119(4), 3410-3423. https://doi.org/10.1002/2013JB010836 
Schultz, R., Stern, V., Gu, Y. J., \& Eaton, D. (2015b). Detection Threshold and Location Resolution of the Alberta Geological Survey Earthquake Catalogue. Seismological Research Letters, 86(2A), 385-397. https://doi.org/10.1785/0220140203

Schultz, R., Stern, V., Novakovic, M., Atkinson, G., \& Gu, Y. J. (2015c). Hydraulic fracturing and the Crooked Lake Sequences: Insights gleaned from regional seismic networks. Geophysical Research Letters, 42(8), 2750-2758. https://doi.org/10.1002/2015GL063455

Schultz, R., Wang, R., Gu, Y. J., Haug, K., \& Atkinson, G. (2017). A seismological overview of the induced earthquakes in the Duvernay play near Fox Creek, Alberta. Journal of Geophysical Research: Solid Earth, 122(1), 492-505. https://doi.org/10.1002/2016JB013570

Schwarz, G. (1978). Estimating the Dimension of a Model. Ann. Statist., 6(2), 461-464. https://doi.org/10.1214/aos/1176344136

Scuderi, M. M., \& Collettini, C. (2016). The role of fluid pressure in induced vs. Triggered seismicity: Insights from rock deformation experiments on carbonates. Scientific Reports, 6, 24852.

Scuderi, M. M., Collettini, C., \& Marone, C. (2017). Frictional stability and earthquake triggering during fluid pressure stimulation of an experimental fault. Earth and Planetary Science Letters, 477(Journal Article), 84-96. https://doi.org/10.1016/j.epsl.2017.08.009

Segall, P. (1985). Stress and subsidence resulting from subsurface fluid withdrawal in the epicentral region of the 1983 Coalinga Earthquake. Journal of Geophysical Research, 90(B8), 6801. https://doi.org/10.1029/JB090iB08p06801

Segall, P. (2010). Earthquake and volcano deformation. Princeton, N.J: Princeton University Press.

Segall, P., Grasso, J., \& Mossop, A. (1995). Poroelastic stressing and induced seismicity near the Lacq gas field, southwestern France. International Journal of Rock Mechanics and Mining Sciences and Geomechanics Abstracts, 32(4), A152-A152. https://doi.org/10.1016/0148-9062(95)96941-4 
Segall, P., \& Lu, S. (2015). Injection-induced seismicity: Poroelastic and earthquake nucleation effects. Journal of Geophysical Research: Solid Earth, 120(7), 5082-5103. https://doi.org/10.1002/2015JB012060

Sereno, T. J., \& Bratt, S. R. (1989). Seismic detection capability at NORESS and implications for the detection threshold of a hypothetical network in the Soviet Union. Journal of Geophysical Research: Solid Earth, 94(B8), 10397-10414. https://doi.org/10.1029/JB094iB08p10397

Shah, A. K., \& Keller, G. R. (2017). Geologic influence on induced seismicity: Constraints from potential field data in Oklahoma: Geologic Influence on Induced Seismicity. Geophysical Research Letters, 44(1), 152-161. https://doi.org/10.1002/2016GL071808

Shcherbakov, R., Goda, K., Ivanian, A., \& Atkinson, G. M. (2013). Aftershock Statistics of Major Subduction Earthquakes. Bulletin of the Seismological Society of America, 103(6), 3222-3234. https://doi.org/10.1785/0120120337

Shcherbakov, R., Nguyen, M., \& Quigley, M. (2012). Statistical analysis of the 2010 M W 7.1 Darfield Earthquake aftershock sequence. New Zealand Journal of Geology and Geophysics, 55(3), 305-311. https://doi.org/10.1080/00288306.2012.676556

Shcherbakov, R., Turcotte, D. L., \& Rundle, J. B. (2004). A generalized Omori’s law for earthquake aftershock decay. Geophysical Research Letters, 31(11), L11613. https://doi.org/10.1029/2004GL019808

Shcherbakov, R., Turcotte, D. L., \& Rundle, J. B. (2005a). Aftershock Statistics. Pure and Applied Geophysics, 162(6), 1051-1076. https://doi.org/10.1007/s00024-004-2661-8

Shcherbakov, R., Turcotte, D. L., \& Rundle, J. B. (2006). Scaling Properties of the Parkfield Aftershock Sequence. Bulletin of the Seismological Society of America, 96(6), 24722472. https://doi.org/10.1785/0120060197

Shcherbakov, R., Yakovlev, G., Turcotte, D. L., \& Rundle, J. B. (2005b). Model for the distribution of aftershock interoccurrence times. Physical Review Letters, 95(21), 218501-218501. https://doi.org/10.1103/PhysRevLett.95.218501

Shi, Y., \& Bolt, B. (1982). The standard error of the magnitude-frequency b value. Bulletin of the Seismological Society of America, 72(5), 1677. 
Skoumal, R. J., Brudzinski, M. R., \& Currie, B. S. (2015). Earthquakes Induced by Hydraulic Fracturing in Poland Township, Ohio. Bulletin of the Seismological Society of America, 105(1), 189-197. https://doi.org/10.1785/0120140168

Skoumal, R. J., Ries, R., Brudzinski, M. R., Barbour, A. J., \& Currie, B. S. (2018). Earthquakes Induced by Hydraulic Fracturing Are Pervasive in Oklahoma. Journal of Geophysical Research: Solid Earth, 123(12), 10,918-10,935. https://doi.org/10.1029/2018JB016790

Smith, M. B., \& Montgomery, C. T. (2015). Hydraulic fracturing. Boca Raton, Florida: CRC Press.

Stein, R. S. (1999). The role of stress transfer in earthquake occurrence. Nature, 402(6762), 605-609. https://doi.org/10.1038/45144

Stein, S., \& Wysession, M. (2003). An introduction to seismology, earthquakes, and earth structure. Malden, MA: Blackwell Pub.

Tinti, S., \& Mulargia, F. (1987). Confidence intervals of b values for grouped magnitudes. Bulletin of the Seismological Society of America, 77(6), 2125-2134.

Udias, A., Madariaga, R., \& Buforn, E. (2013). Source mechanisms of earthquakes: Theory and practice. Source Mechanisms of Earthquakes: Theory and Practice, 302. https://doi.org/10.1017/CBO9781139628792

Utsu, T. (1961). A statistical study of the occurrence of aftershocks. Geophysical Magazine, (30), 521-605.

Utsu, T. (1966). A Statistical Significance Test of the Difference in b-value between Two Earthquake Groups. Journal of Physics of the Earth, 14(2), 37-40. https://doi.org/10.4294/jpe1952.14.37

Utsu, T. (1970). Aftershocks and earthquake statistics (II)—Further investigation of aftershocks and other earthquake sequences based on a new classification of earthquake sequences. J. Fac. Sci. Hokkaido Univ., 3, 197-266.

van der Elst, N., Keranen, K. M., \& Abers, G. A. (2013). Enhanced Remote Earthquake Triggering at Fluid-Injection Sites in the Midwestern United States. Science, 341(6142), 164-167. https://doi.org/10.1126/science.1238948

van Stiphout, T., Kissling, E., Wiemer, S., \& Ruppert, N. (2009). Magmatic processes in the Alaska subduction zone by combined 3-D b value imaging and targeted seismic 
tomography. Journal of Geophysical Research: Solid Earth, 114(B11).

https://doi.org/10.1029/2008JB005958

Vasylkivska, V. S., \& Huerta, N. J. (2017). Spatiotemporal distribution of Oklahoma earthquakes: Exploring relationships using a nearest-neighbor approach. Journal of Geophysical Research: Solid Earth, 122(7), 5395-5416. https://doi.org/10.1002/2016JB013918

Vermylen, J., \& Zoback, M. D. (2011). Hydraulic fracturing, microseismic magnitudes and stress evolution in the Barnett Shale, Texas, USA. Presented at the Hydraulic Fracturing Technology Conference and Exhibition, Society of Petroleum Engineers.

Vidale, J. E., \& Shearer, P. M. (2006). A survey of 71 earthquake bursts across southern California: Exploring the role of pore fluid pressure fluctuations and aseismic slip as drivers. Journal of Geophysical Research - Solid Earth, 11 (B5), B05312. https://doi.org/10.1029/2005JB004034

Wang, R., Gu, Y. J., Schultz, R., Kim, A., \& Atkinson, G. (2016). Source analysis of a potential hydraulic-fracturing-induced earthquake near Fox Creek, Alberta. Geophysical Research Letters, 43(2), 564. https://doi.org/10.1002/2015GL066917

Wang, R., \& Kümpel, H. (2003). Poroelasticity: Efficient modeling of strongly coupled, slow deformation processes in a multilayered half-space. Geophysics, 68(2), 705-717. https://doi.org/10.1190/1.1567241

Wetmiller, R. J. (1986). Earthquakes near Rocky Mountain House, Alberta, and their relationship to gas production facilities. Canadian Journal of Earth Sciences, 23(2), 172-181. https://doi.org/10.1139/e86-020

Wiemer, S., \& Schorlemmer, D. (2007). ALM: An Asperity-based Likelihood Model for California. Seismological Research Letters, 78(1), 134-140. https://doi.org/10.1785/gssrl.78.1.134

Wiemer, S., \& Wyss, M. (1997). Mapping the frequency-magnitude distribution in asperities: An improved technique to calculate recurrence times? Journal of Geophysical Research: Solid Earth, 102(B7), 15115-15128. https://doi.org/10.1029/97JB00726

Wiemer, S., \& Wyss, M. (2000). Minimum Magnitude of Completeness in Earthquake Catalogs: Examples from Alaska, the Western United States, and Japan. Bulletin of the Seismological Society of America, 90(4), 859-869. https://doi.org/10.1785/0119990114 
Woessner, J., \& Wiemer, S. (2005). Assessing the Quality of Earthquake Catalogues: Estimating the Magnitude of Completeness and Its Uncertainty. Bulletin of the Seismological Society of America, 95(2), 684-698. https://doi.org/10.1785/0120040007

Wyss, M., Wiemer, S., \& Schorlemmer, D. (2005). Variations in earthquake-size distribution across different stress regimes. Nature, 437(7058), 539-542. https://doi.org/10.1038/nature04094

Zaliapin, I., \& Ben-Zion, Y. (2013a). Earthquake clusters in southern California I: Identification and stability. Journal of Geophysical Research: Solid Earth, 118(6), 2847-2864. https://doi.org/10.1002/jgrb.50179

Zaliapin, I., \& Ben-Zion, Y. (2013b). Earthquake clusters in southern California II: Classification and relation to physical properties of the crust. Journal of Geophysical Research: Solid Earth, 118(6), 2865-2877. https://doi.org/10.1002/jgrb.50178

Zaliapin, I., \& Ben-Zion, Y. (2016). Discriminating Characteristics of Tectonic and HumanInduced Seismicity. Bulletin of the Seismological Society of America, 106(3), 846-859. https://doi.org/10.1785/0120150211

Zaliapin, I., Gabrielov, A., Keilis-Borok, V., \& Wong, H. (2008). Clustering analysis of seismicity and aftershock identification. Physical Review Letters, 101(1), 018501. https://doi.org/10.1103/PhysRevLett.101.018501

Zhang, H., Eaton, D. W., Rodriguez, G., \& Jia, S. Q. (2019). Source-Mechanism Analysis and Stress Inversion for Hydraulic-Fracturing-Induced Event Sequences near Fox Creek, Alberta. Bulletin of the Seismological Society of America, 109(2), 636-651. https://doi.org/10.1785/0120180275

Zhuang, J., Ogata, Y., \& Vere-Jones, D. (2004). Analyzing earthquake clustering features by using stochastic reconstruction. Journal of Geophysical Research - Solid Earth, 109(B5), B05301-n/a. https://doi.org/10.1029/2003JB002879

Zuniga, F. R., \& Wyss, M. (2001). Most- and Least-Likely Locations of Large to Great Earthquakes along the Pacific Coast of Mexico Estimated from Local Recurrence Times Based on b-Values. Bulletin of the Seismological Society of America, 91(6), 1717-1728. https://doi.org/10.1785/0120000303 


\section{Appendices}

\section{Appendix A: Nearest-Neighbour Distance (NND) distributions and corresponding Gaussian mixture model (GMM) results of the regional WCSB catalogue (1975-2018) for different cut-off magnitudes.}

Through this appendix, I provide figures that illustrate the stability of the NND method with respect to the magnitude cut-off $M_{0}$. Further, a table containing the results of the GMM application for each cut-off is included. It is shown below that the tri-modality observed in the regional distributions in Chapter 3.1 (for $M_{0}=2.0$ ) holds for cut-offs up to $M_{0}=3.0$, which is the estimated regional completeness. Visually, the joint distributions (panels a in the figures below) lose modality at $M_{0}=2.8$ while the one-dimensional $\eta$ distributions (panels $\mathrm{b}$ ) maintain discernible modality up to $M_{0}=3.0$. The Expectation Maximization (EM) algorithm computes minimized Akaike Information Criteria (AIC) for a 3-component GMM for all tested cut-off values and computes minimized Bayesian Information Criteria (BIC) for a 3-component GMM up to $M_{0}=2.6$ (Table A 1$)$. 


$$
M_{0}=0.5
$$

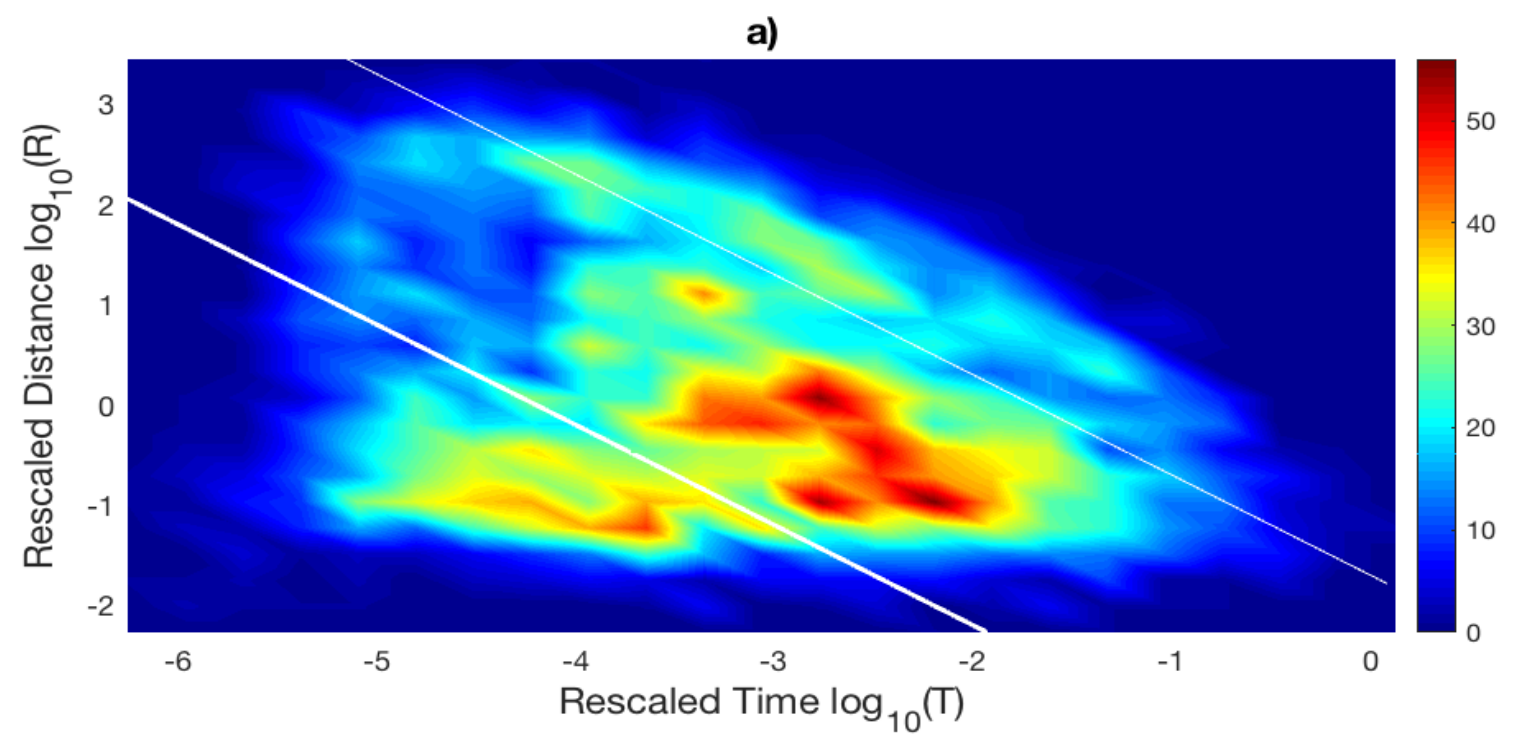

b)

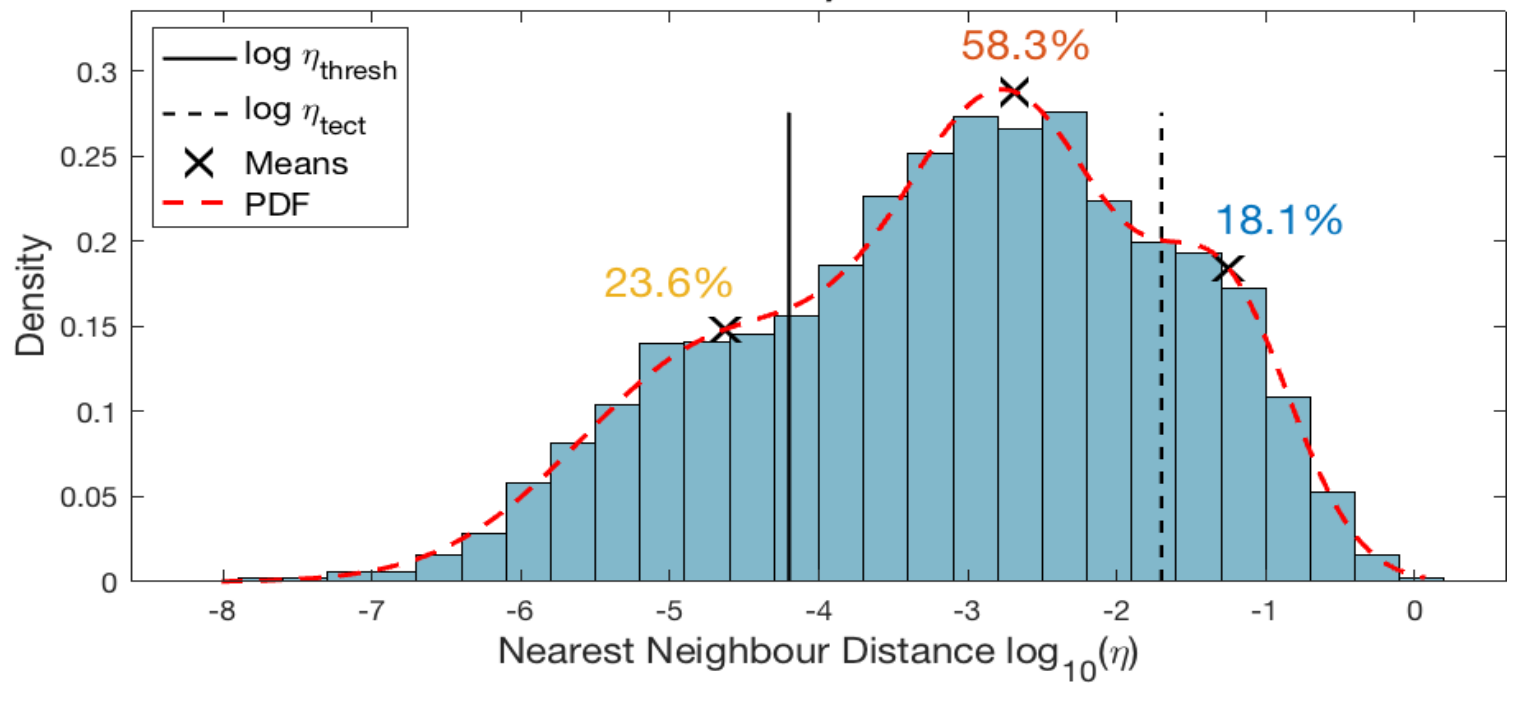

Figure A 1: $M_{0}=0.5$. a) Joint NND (T, R) distribution. Bold white line indicates the threshold $\log _{10} \eta_{\text {thresh }}=-4.2$ between loosely and tightly clustered components. Thin white line indicates the threshold $\log _{10} \eta_{\text {tect }}=-1.7$ between loosely clustered and deepbackground components. Colour bar indicates frequency of occurrence. b) Normalized density of $\eta$ values. Solid black line is $\log _{10} \eta_{\text {thresh }}$ and dashed black line is $\log _{10} \eta_{\text {tect }}$. Dashed red line is the normalized probability density function of the Gaussian mixture and black crosses are the component means. Percentages reflect the modal mixing proportions. 


$$
M_{0}=1.0
$$

a)

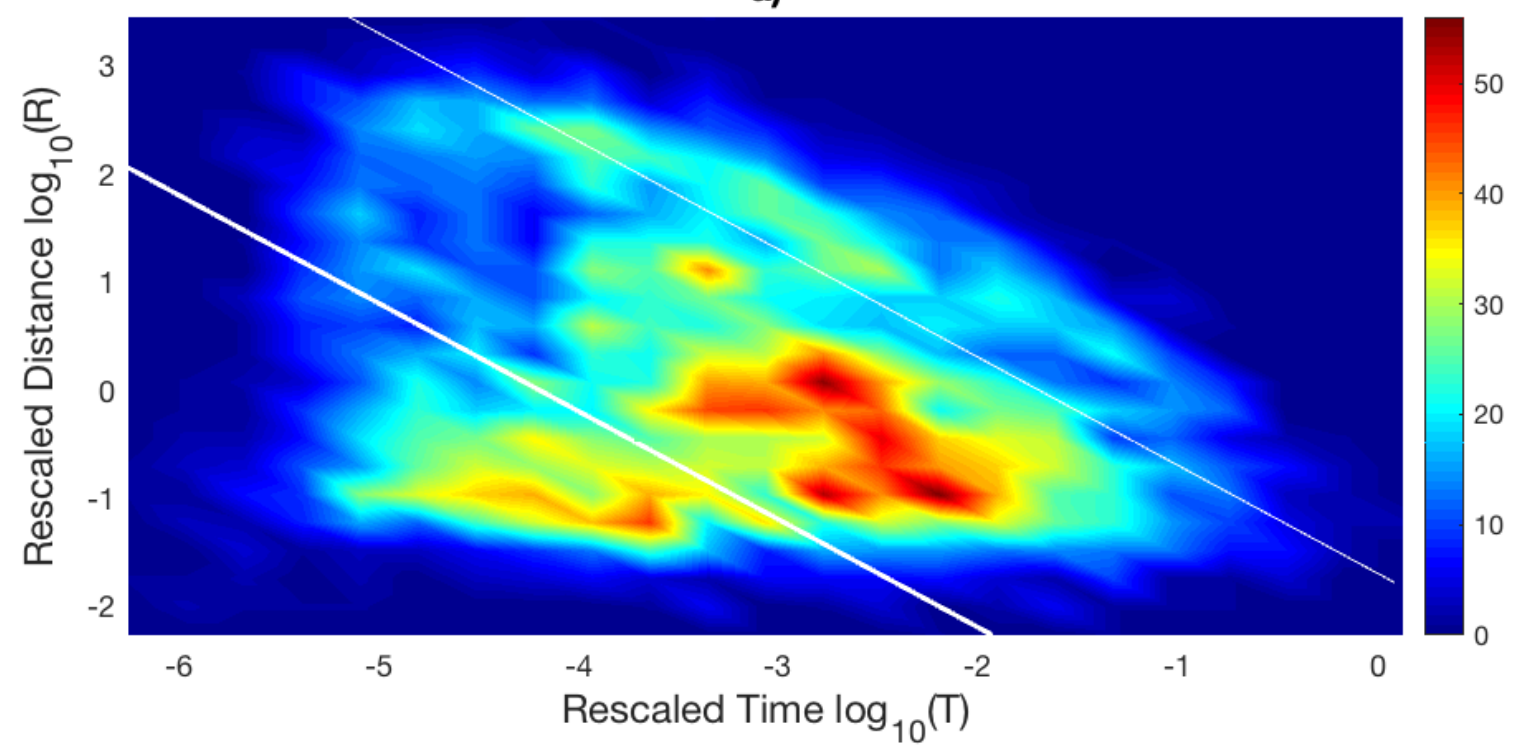

b)

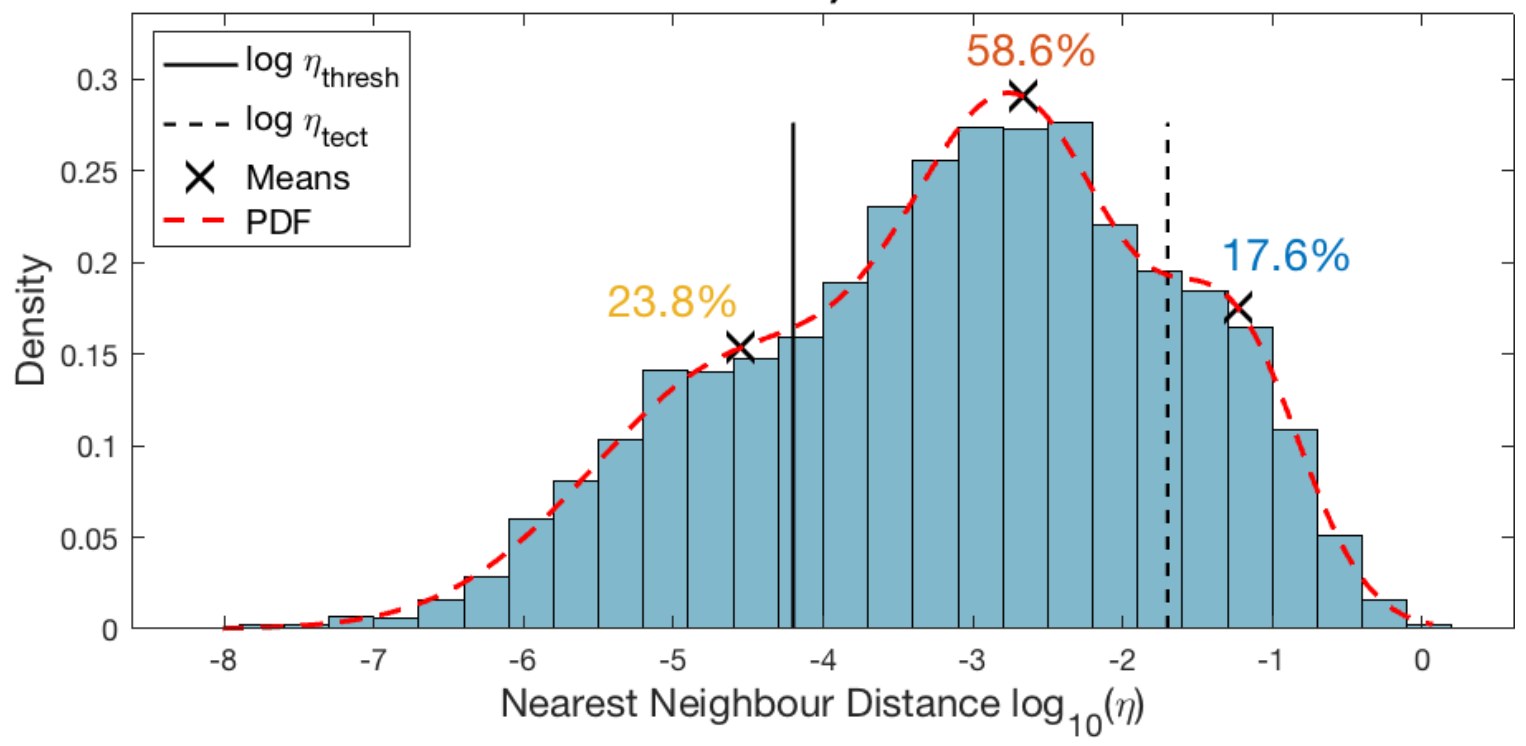

Figure A 2: $M_{0}=1.0$. a) Joint NND $(T, R)$ distribution. b) Normalized density of $\eta$ values. See Figure A 1 caption for a graphical description. 


$$
M_{0}=2.0
$$

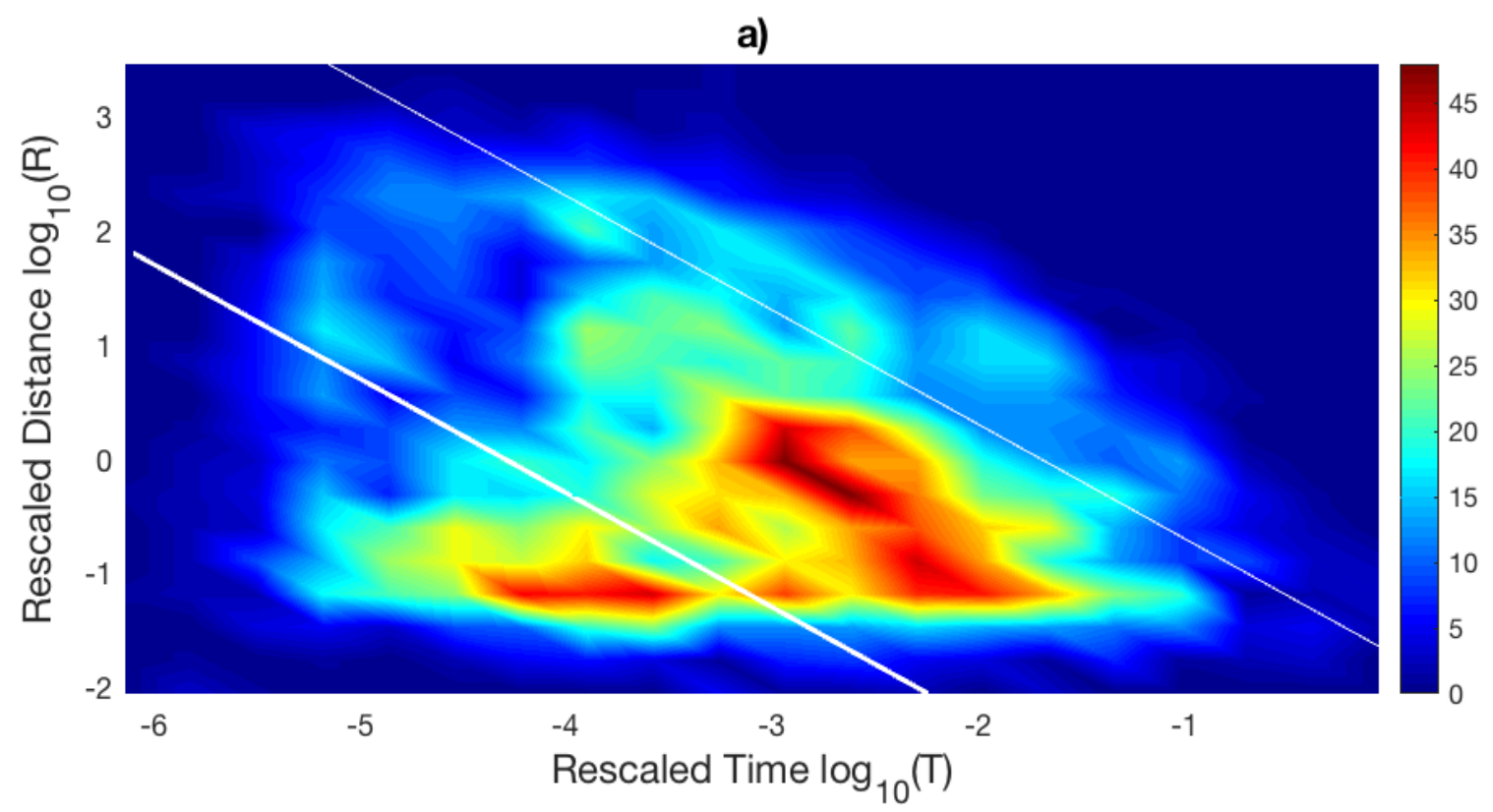

b)

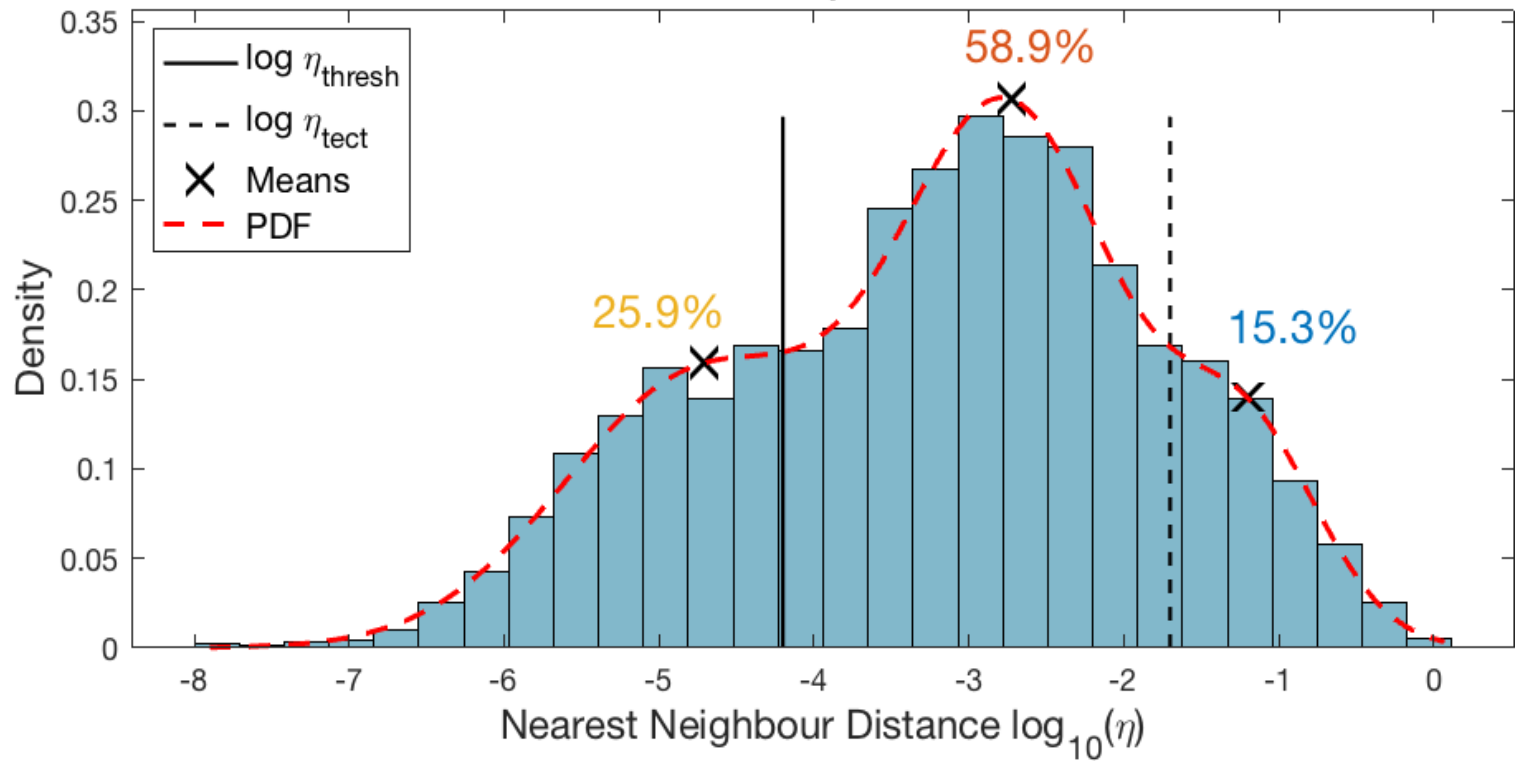

Figure A 3: $M_{0}=2.0$. a) Joint NND $(T, R)$ distribution. b) Normalized density of $\eta$ values. See Figure A 1 caption for a graphical description. 


$$
M_{0}=2.2
$$

a)

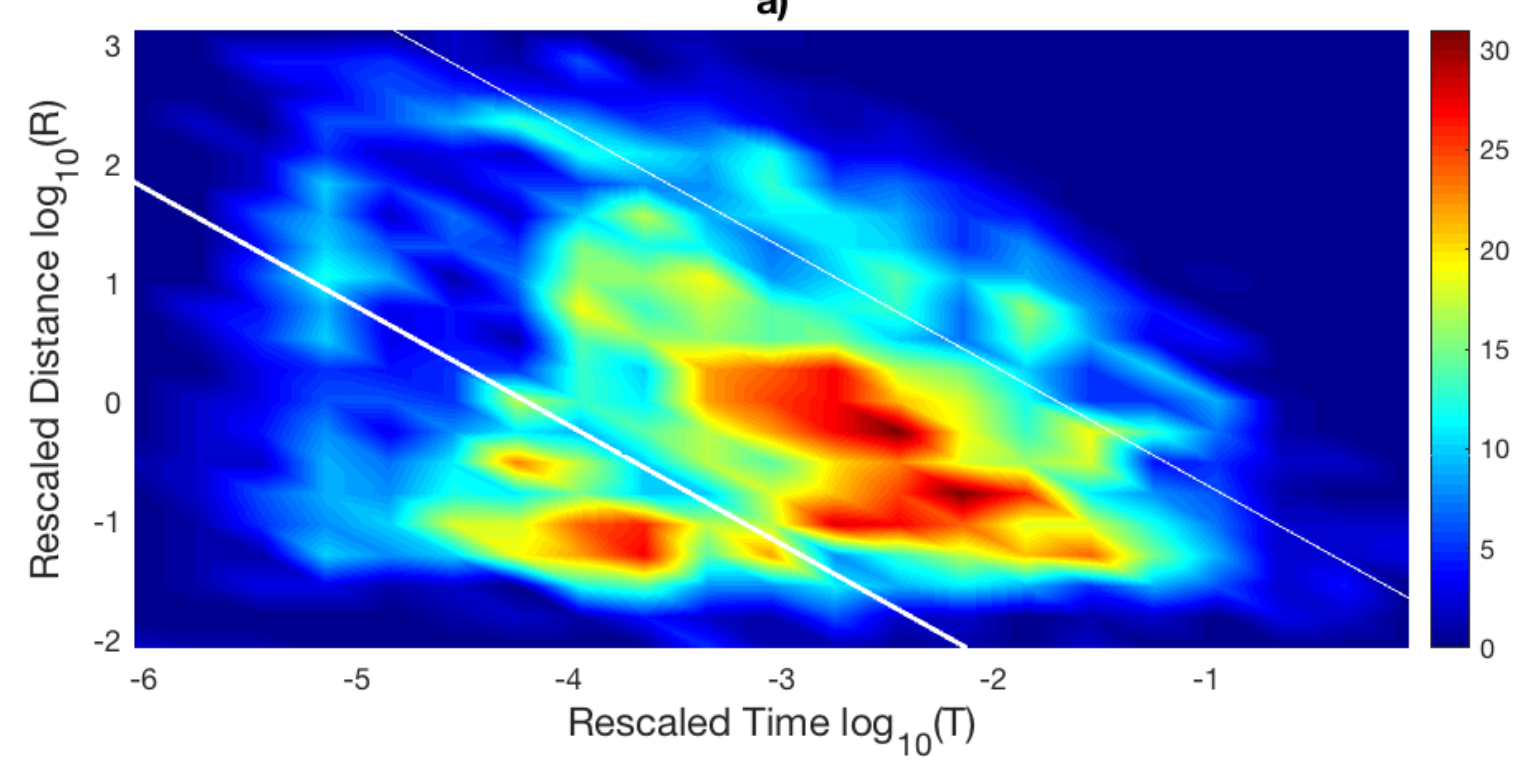

b)

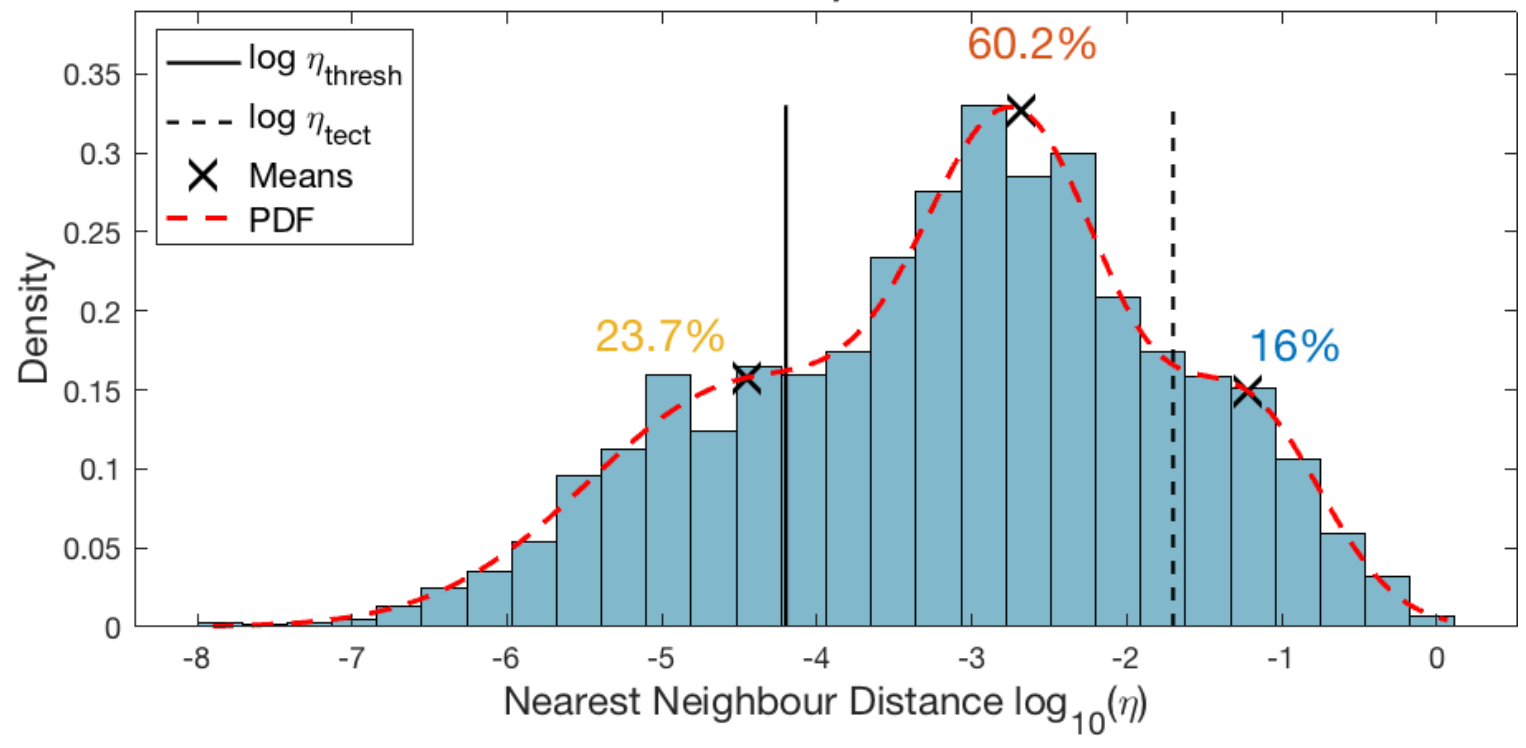

Figure A 4: $M_{0}=2.2$. a) Joint NND $(T, R)$ distribution. b) Normalized density of $\eta$ values. See Figure A 1 caption for a graphical description. 


$$
M_{0}=2.4
$$

a)

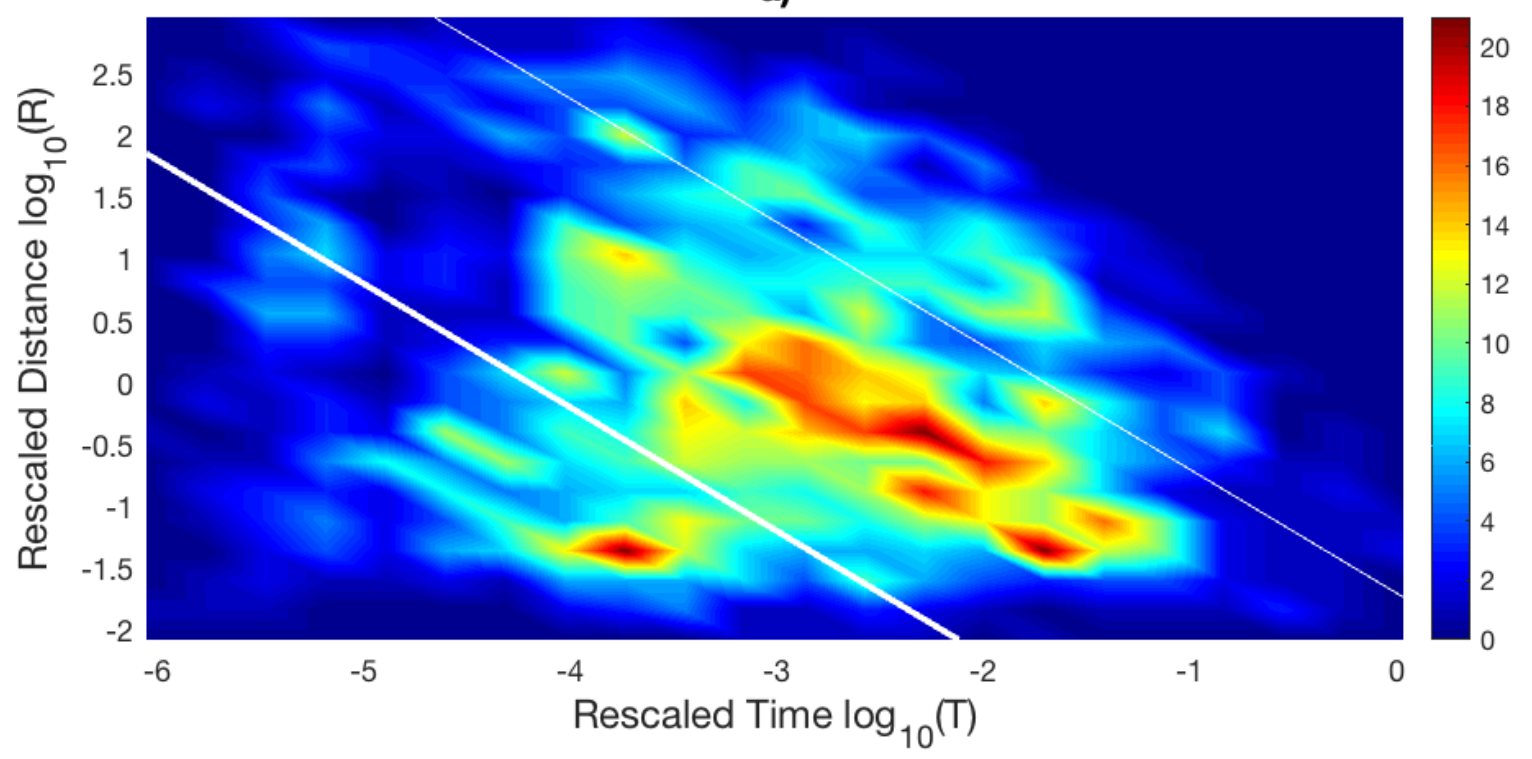

b)

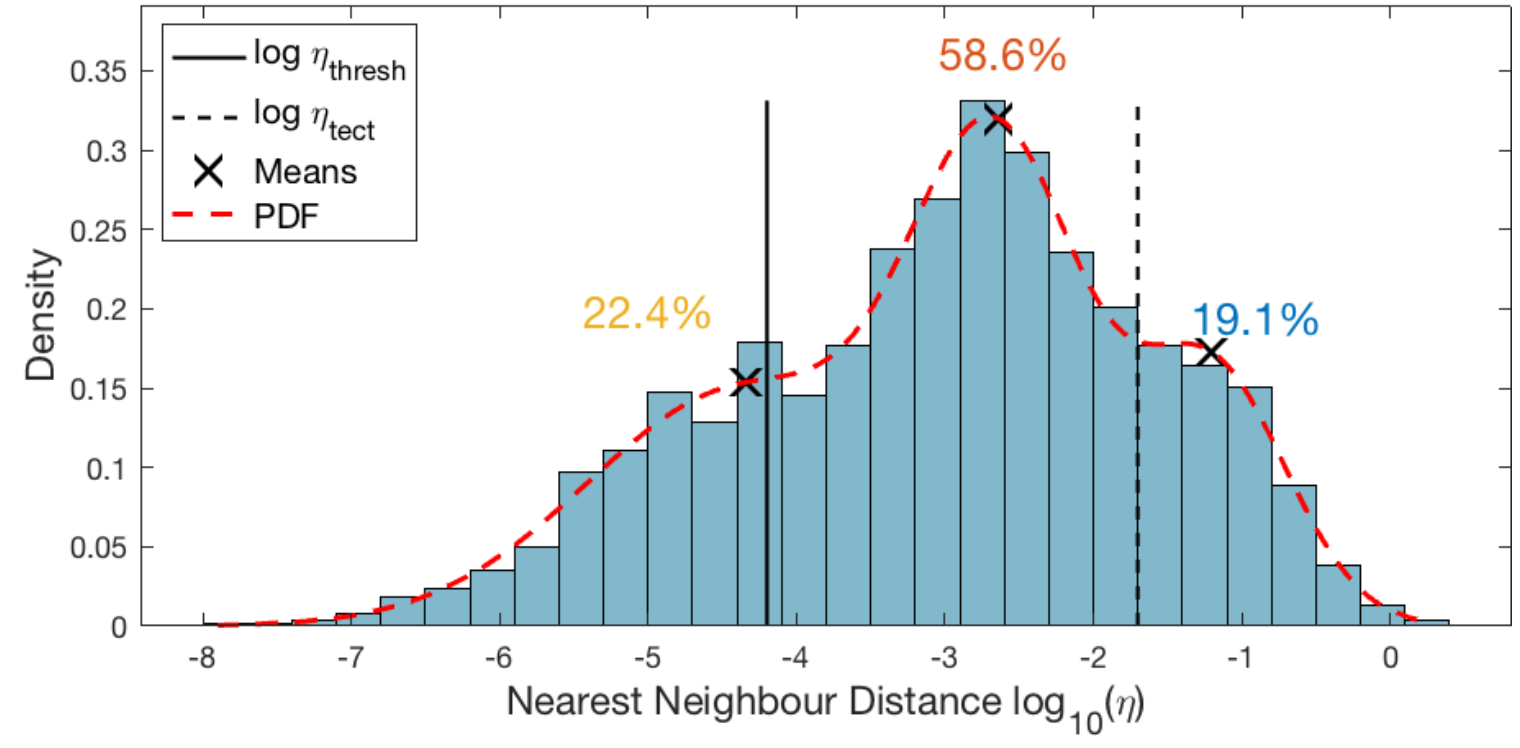

Figure A 5: $M_{0}=2.4$. a) Joint NND $(T, R)$ distribution. b) Normalized density of $\eta$ values. See Figure A 1 caption for a graphical description. 


$$
M_{0}=2.6
$$

a)

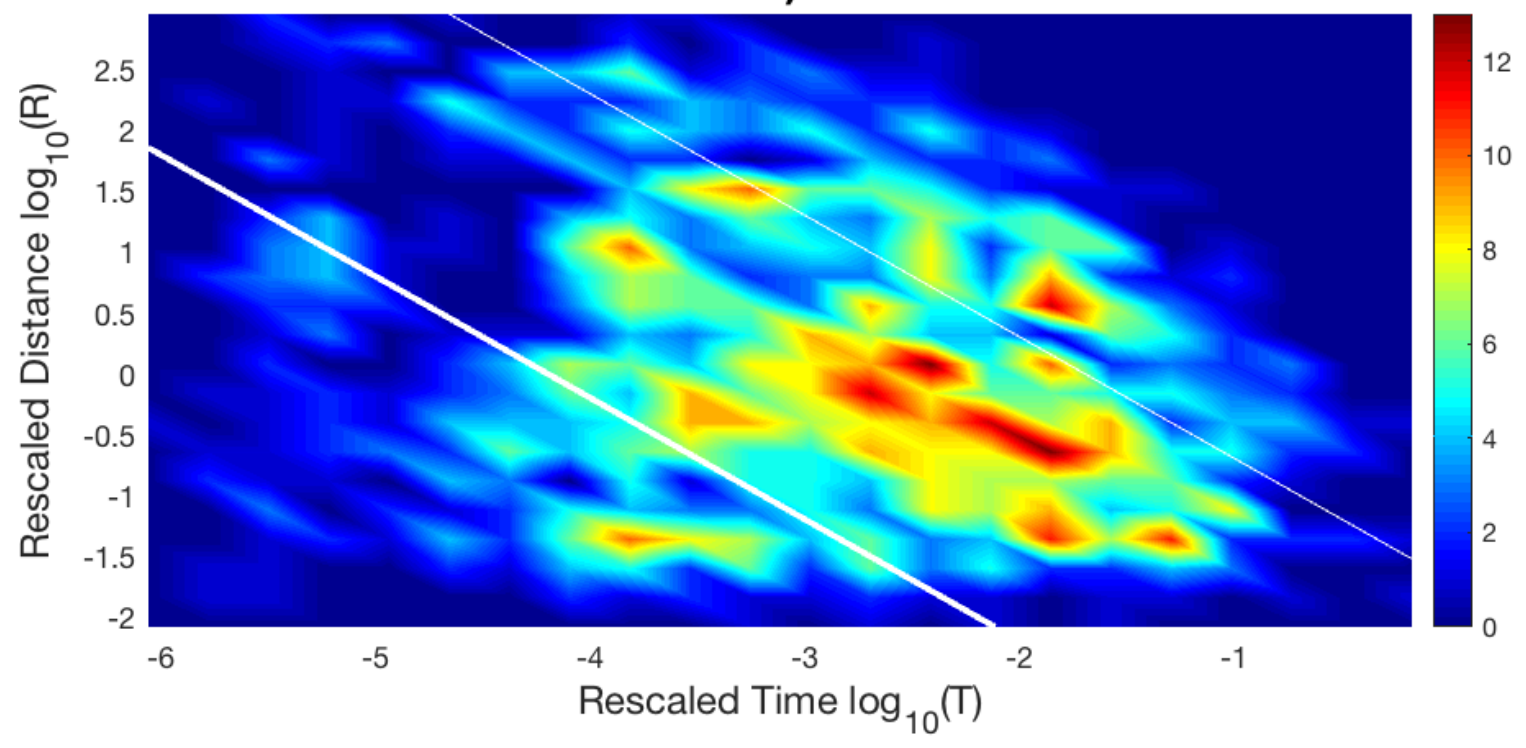

b)

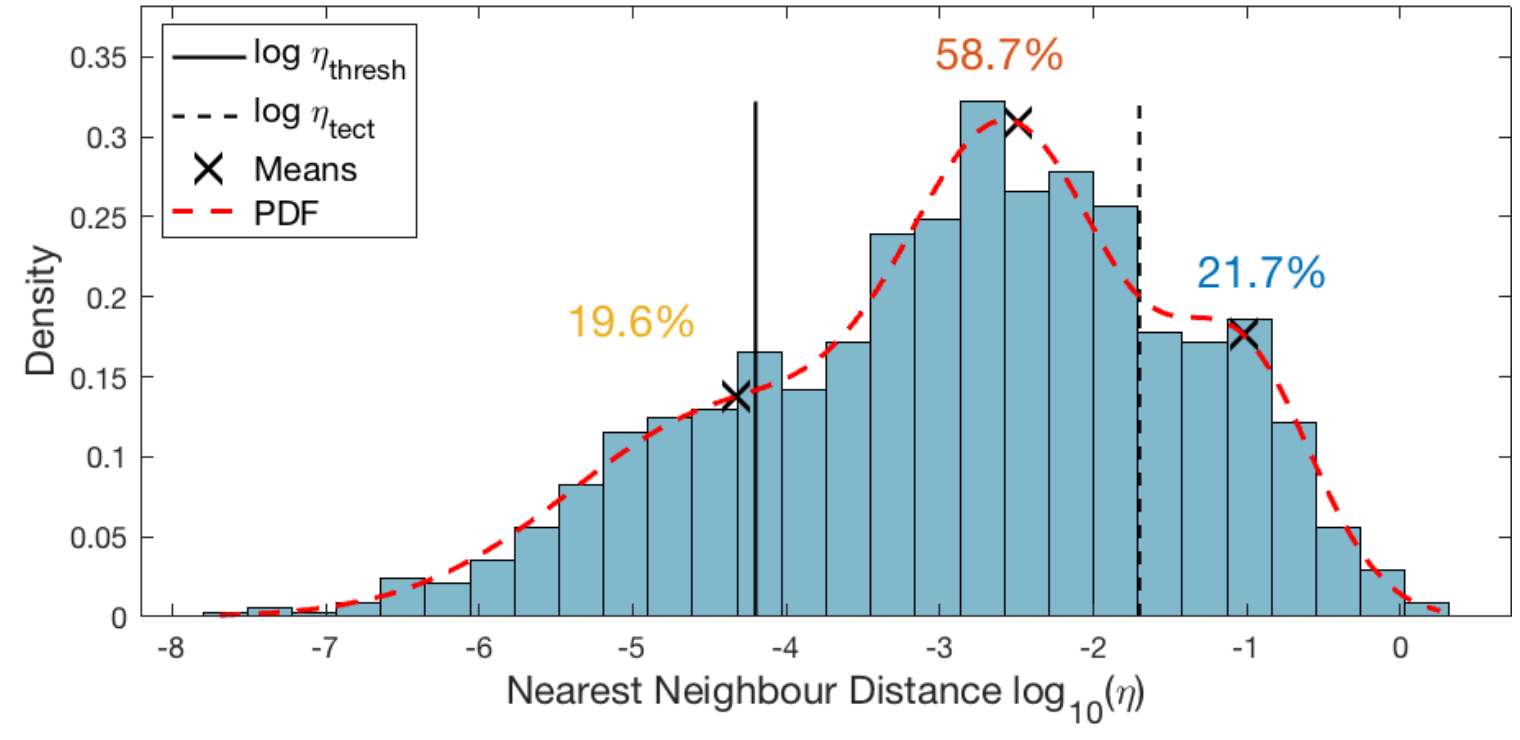

Figure A 6: $M_{0}=2.6$. a) Joint NND $(T, R)$ distribution. b) Normalized density of $\eta$ values. See Figure A 1 caption for a graphical description. 


$$
M_{0}=2.8
$$

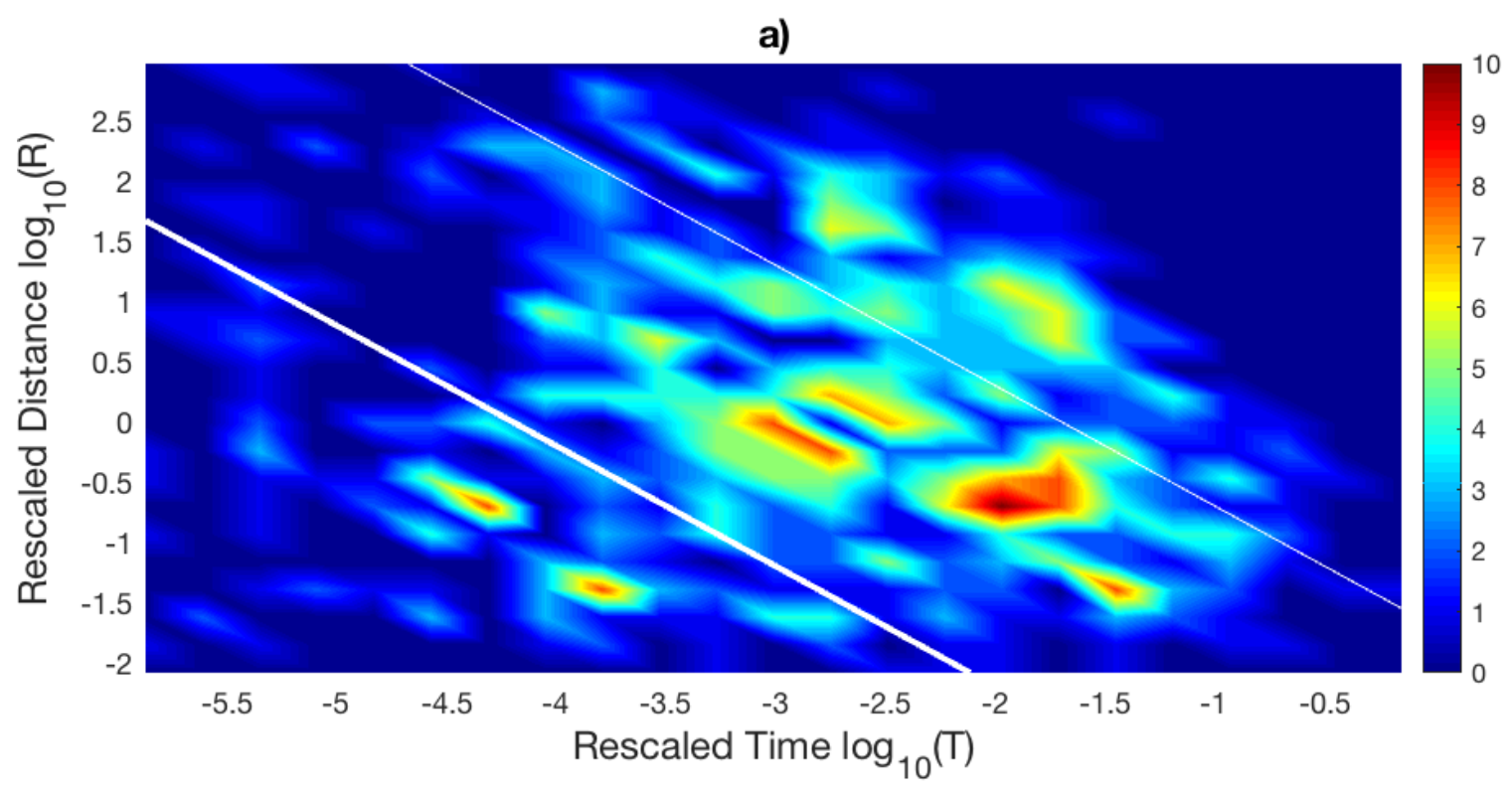

b)

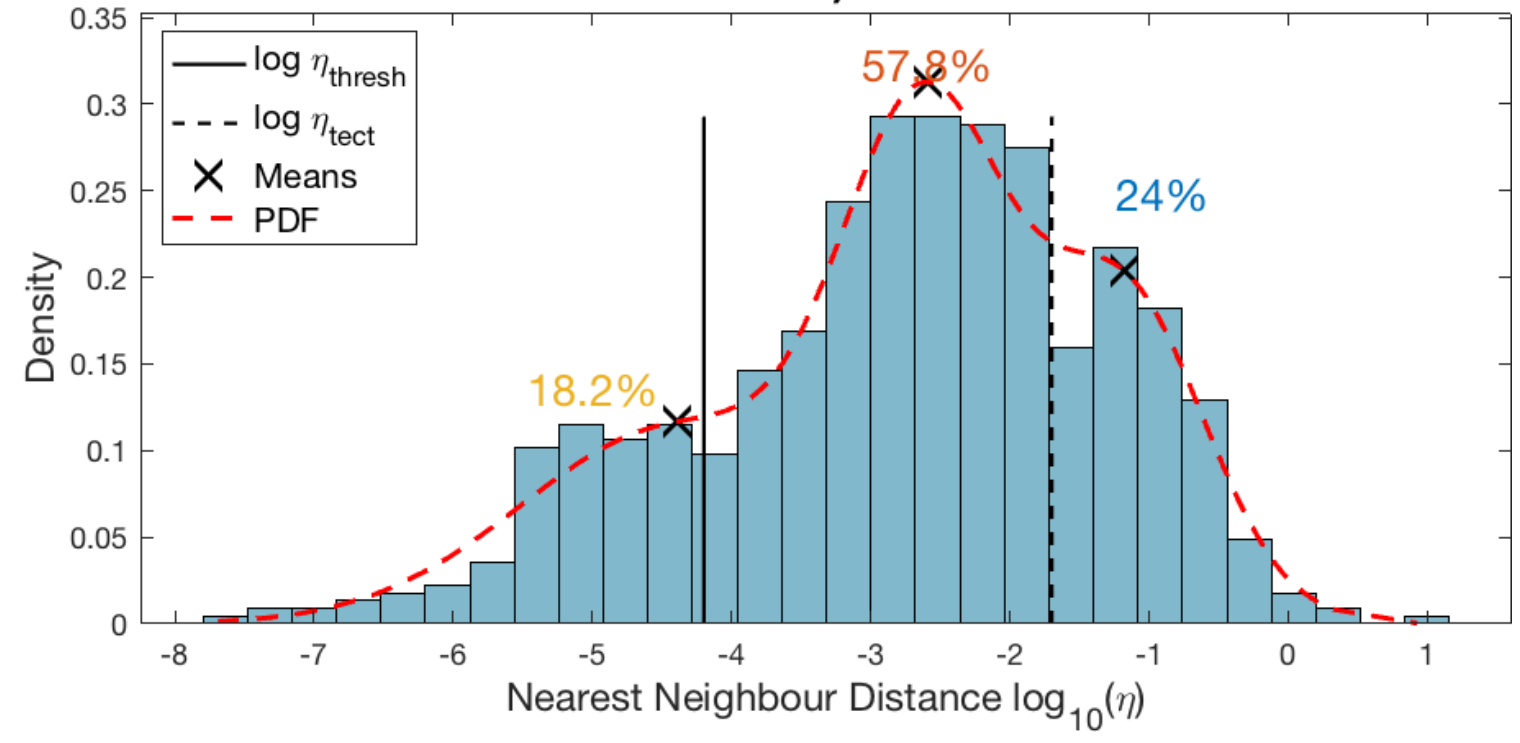

Figure A 7: $M_{0}=2.8$. a) Joint NND $(T, R)$ distribution. b) Normalized density of $\eta$ values. See Figure A 1 caption for a graphical description. 


$$
M_{0}=3.0
$$

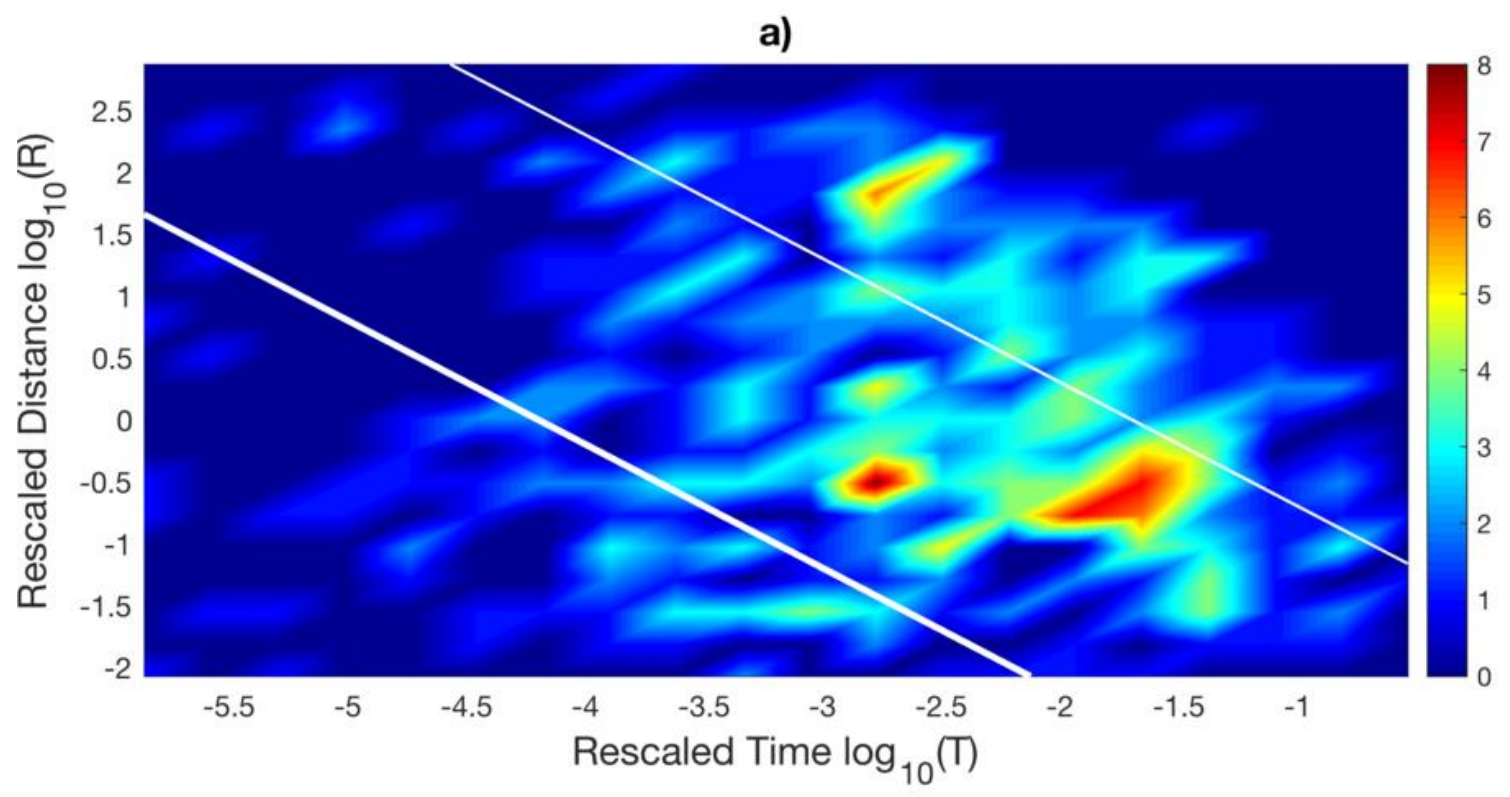

b)

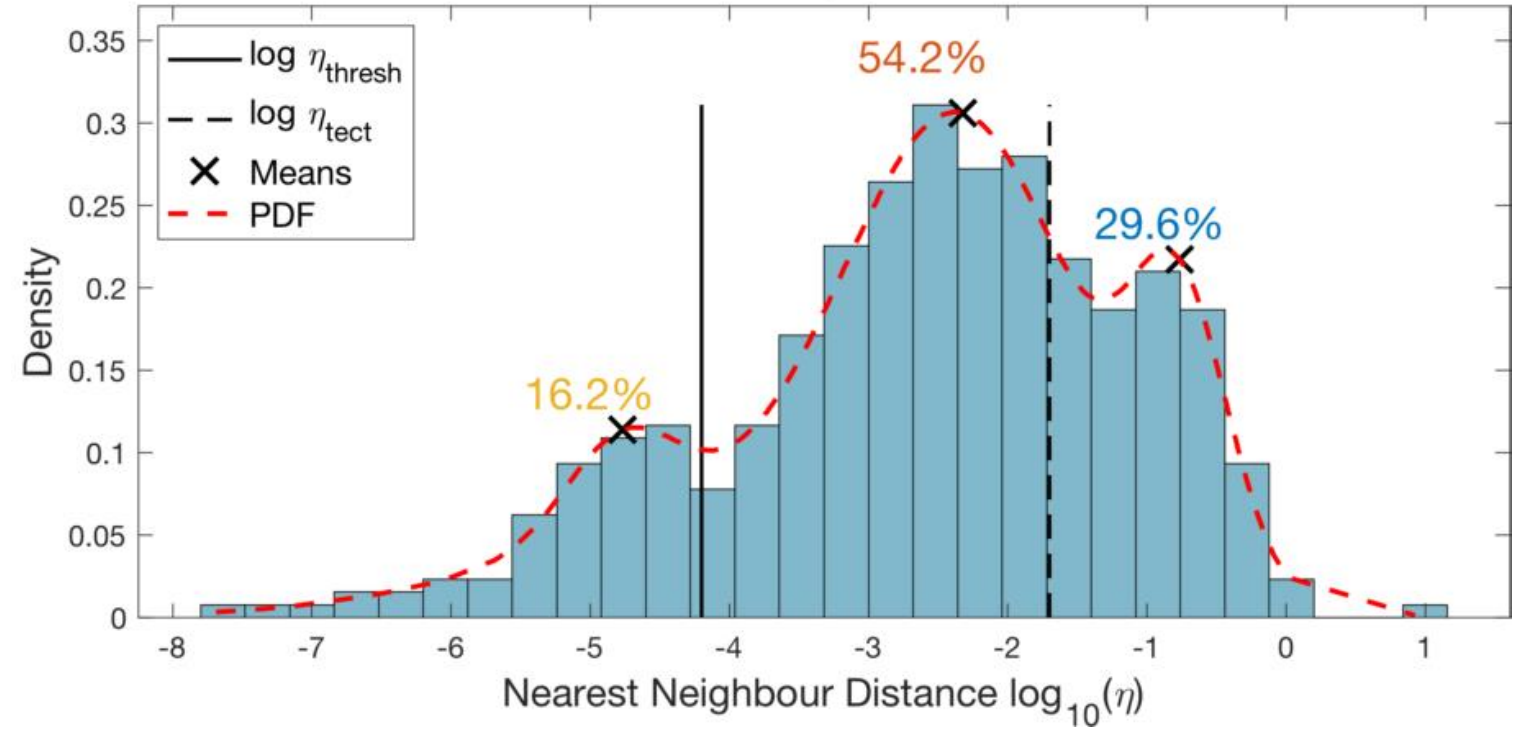

Figure A 8: $M_{0}=3.0$. a) Joint NND $(T, R)$ distribution. b) Normalized density of $\eta$ values. See Figure A 1 caption for a graphical description. 
Table A 1: Expectation Maximization results for the GMM applied to the WCSB regional catalogue (1975-2018) using different cut-off magnitudes $M_{0} . k$ is the number of modes, AIC and BIC are the Akaike and Bayes Information Criteria. Highlights show the minimized values.

\begin{tabular}{|c|c|c|c|c|c|c|c|c|}
\hline$M_{0}$ & 0.5 & 1 & 2 & 2.2 & 2.4 & 2.6 & 2.8 & 3.0 \\
\hline $\mathbf{k}$ & \multicolumn{8}{|c|}{ AIC } \\
\hline 1 & 18933.70 & 18430.44 & 12477.40 & 9400.24 & 6447.10 & 4158.82 & 2547.36 & 1459.52 \\
\hline 2 & 18645.24 & 18173.78 & 12319.62 & 9297.45 & 6374.96 & 4105.84 & 2505.74 & 1434.75 \\
\hline 3 & 18535.38 & 18070.23 & 12270.62 & 9246.28 & 6334.74 & 4084.36 & 2497.49 & 1427.14 \\
\hline 4 & 18548.39 & 18077.51 & 12272.82 & 9249.94 & 6339.42 & 4090.60 & 2499.64 & 1428.57 \\
\hline $\mathbf{k}$ & \multicolumn{8}{|c|}{ BIC } \\
\hline 1 & 18946.87 & 18443.55 & 12489.74 & 9412.03 & 6458.10 & 4168.94 & 2556.47 & 1467.51 \\
\hline 2 & 18678.15 & 18206.55 & 12350.47 & 9326.90 & 6402.46 & 4131.15 & 2528.51 & 1454.72 \\
\hline 3 & 18588.03 & 18122.67 & 12319.97 & 9293.41 & 6378.75 & 4124.85 & 2533.93 & 1459.10 \\
\hline 4 & 18607.79 & 18149.62 & 12340.68 & 9314.74 & 6399.93 & 4146.28 & 2549.75 & 1472.50 \\
\hline
\end{tabular}




\section{Appendix B: Nearest-Neighbour Distance (NND) distributions and corresponding Gaussian mixture model (GMM) results of the regional WCSB catalogue for separated time periods (1975-2009 and 2010-2018) using different cut-off magnitudes.}

The figures and tables within this appendix demonstrate that the fundamental changes observed within the regional NND distributions over time in Chapter 3.2 (for $M_{0}=2.0$ ), specifically the sharp transition from background-dominant seismicity to loosely and tightly clustered seismicity, hold for cut-offs up to $M_{0}=3.0$, which is the estimated regional completeness level. Over the first time frame, the Expectation Maximization (EM) algorithm consistently identifies 3 modes (shown by the black crosses in panels c of the figures below). Over the second time frame it mainly identifies two components, the loosely and tightly clustered modes (black crosses in panels $\mathrm{d}$ of the figures below), indicating the dominant proportions of these earthquake subpopulations over recent years. A 10-15\% mixing proportion increase in both clustered subpopulations between time periods is maintained for cut-offs up to $M_{0}=3.0$, as is a $20-30 \%$ decrease in deep-background proportion. These results suggest that the changes observed over time cannot be fully explained by improvements in network detection capability alone. 


$$
M_{0}=0.5
$$

a)

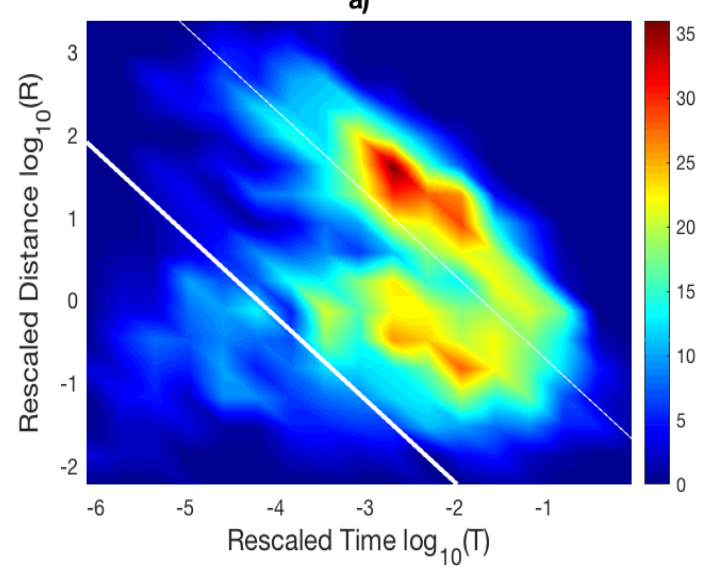

c)

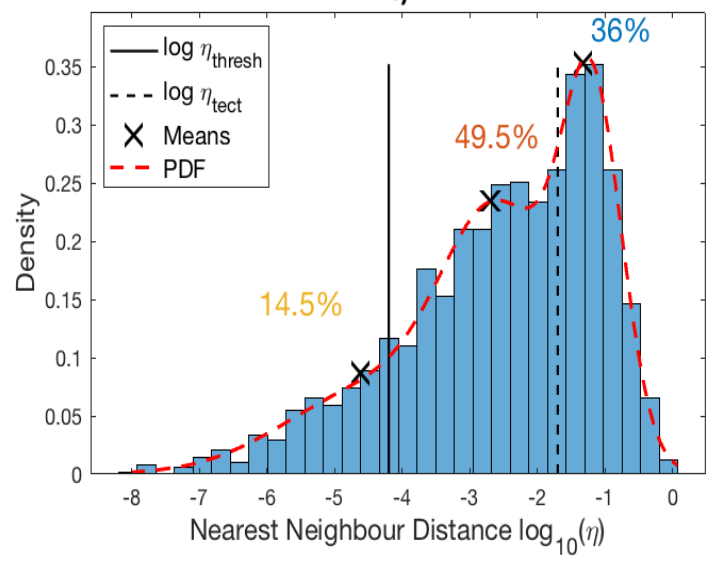

b)

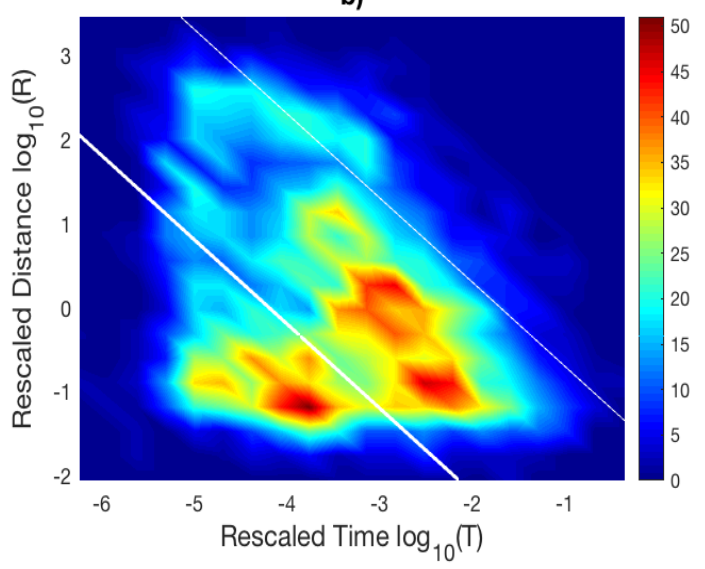

d)

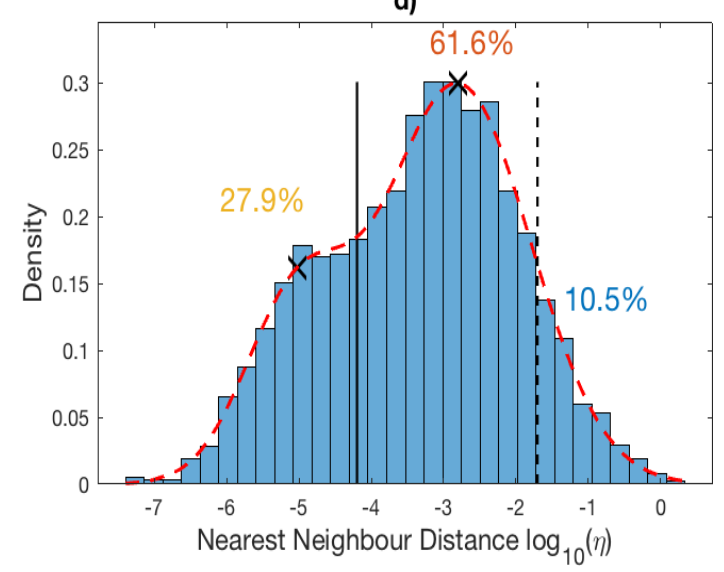

Figure B 1: $M_{0}=0.5$. (a, c) 1975-2009. (b, d) 2010-2018. (a, b) Joint distributions of the temporal and spatial components $(T, R)$. Bold white lines indicate the threshold $\log _{10} \eta_{\text {thresh }}=-4.2$ between loosely and tightly clustered components. Thin white lines indicate the threshold $\log _{10} \eta_{\text {tect }}=-1.7$ between loosely clustered and deep-background components. Colour bars indicate frequency of occurrence. (c, d) Normalized densities of $\eta$ values. Solid black lines are $\log _{10} \eta_{\text {thresh }}$ and dashed black lines are $\log _{10} \eta_{\text {tect }}$. Dashed red lines are the normalized probability density functions of the Gaussian mixtures and black crosses are the component means. Percentages reflect the modal mixing proportions. 


$$
M_{0}=1.0
$$
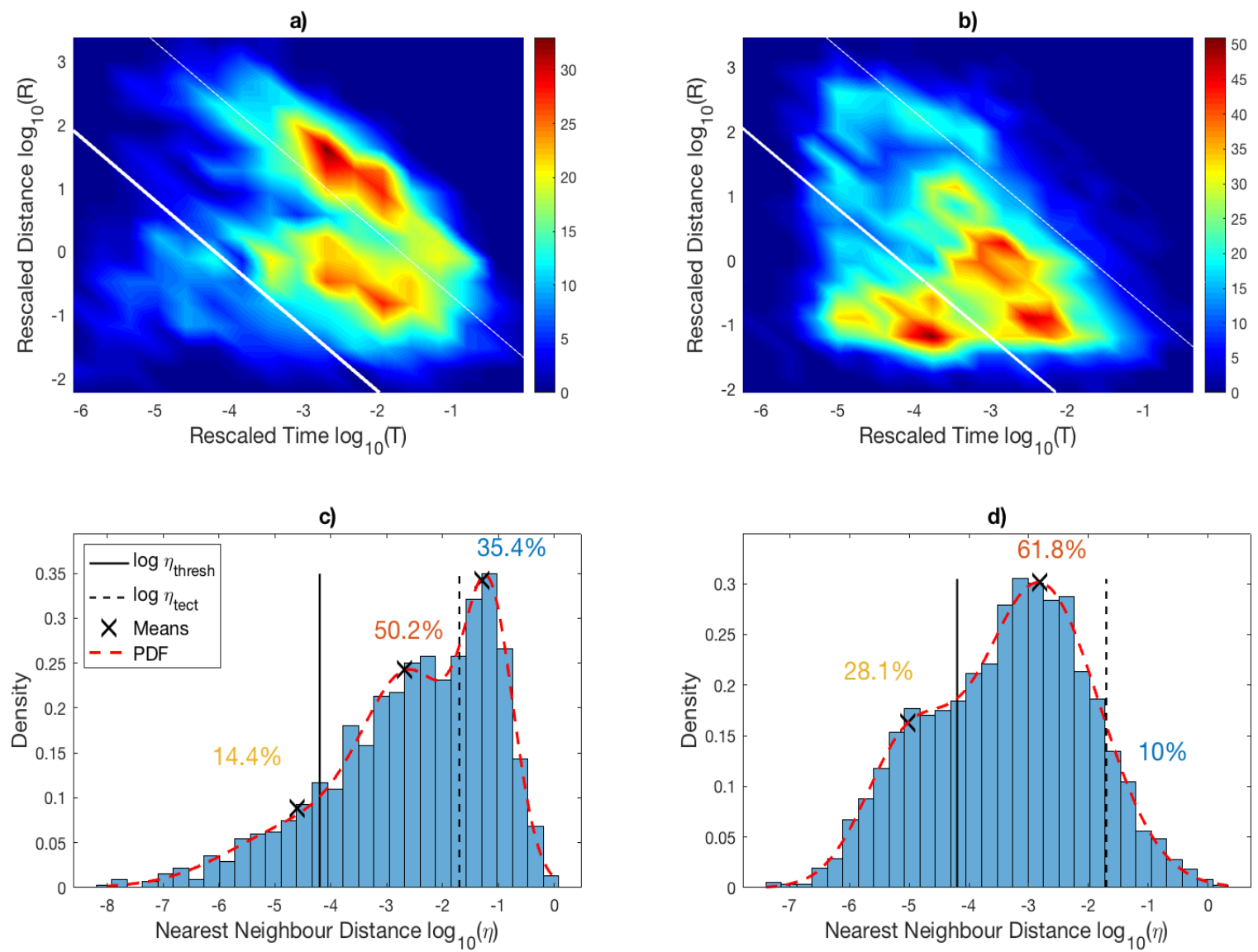

Figure B 2: $M_{0}=1.0$. (a, c) 1975-2009. (b, d) 2010-2018. (a, b) Joint distributions of the temporal and spatial components $(T, R)$. (c, d) Normalized densities of $\eta$ values. See Figure B 1 caption for a graphical description. 


$$
M_{0}=2.0
$$

a)

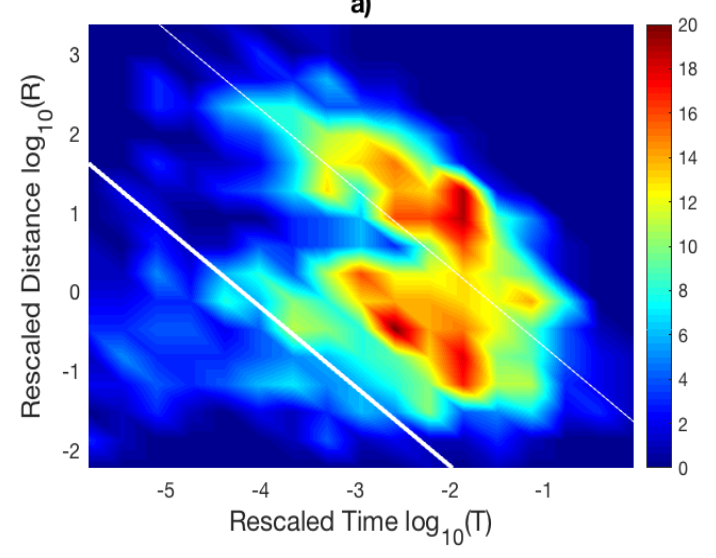

c)

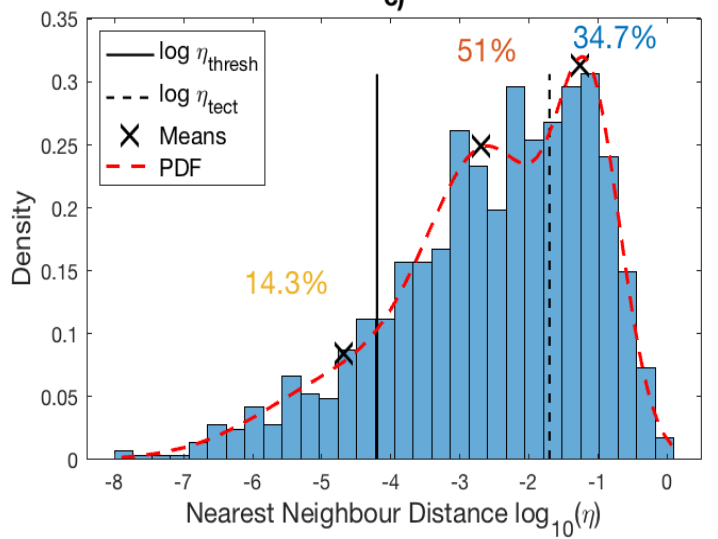

b)

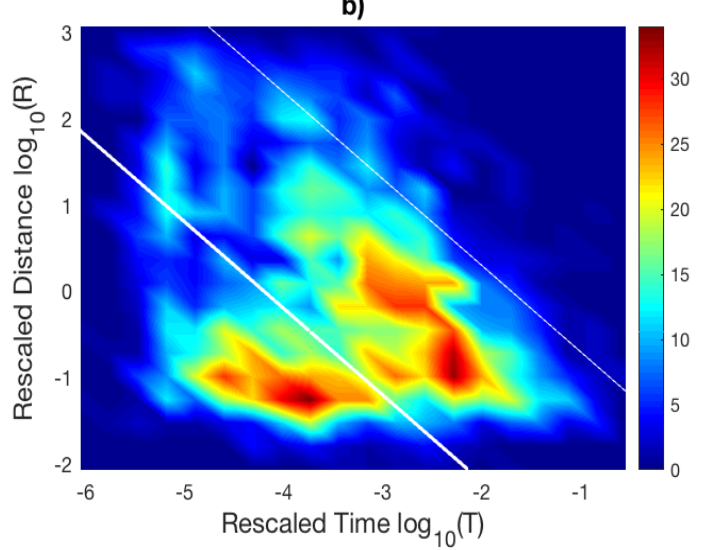

d)

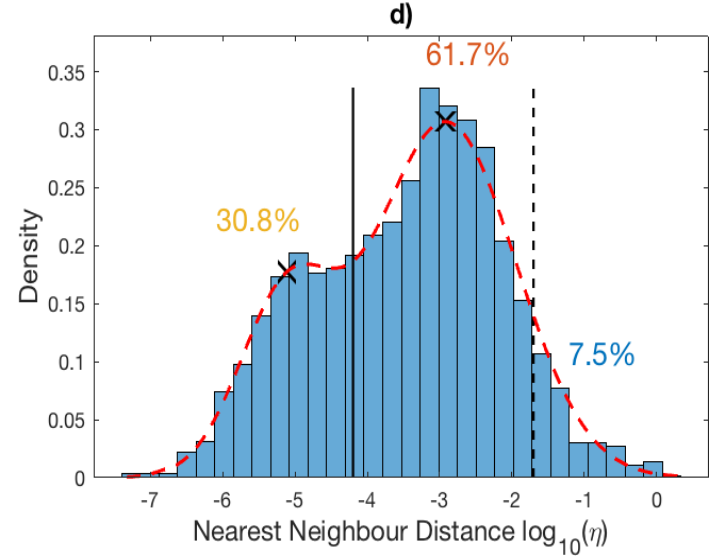

Figure B 3: $M_{0}=2$. 0. (a, c) 1975-2009. (b, d) 2010-2018. (a, b) Joint distributions of the temporal and spatial components $(T, R)$. (c, d) Normalized densities of $\eta$ values. See Figure B 1 caption for a graphical description. 
$M_{0}=2.2$

a)

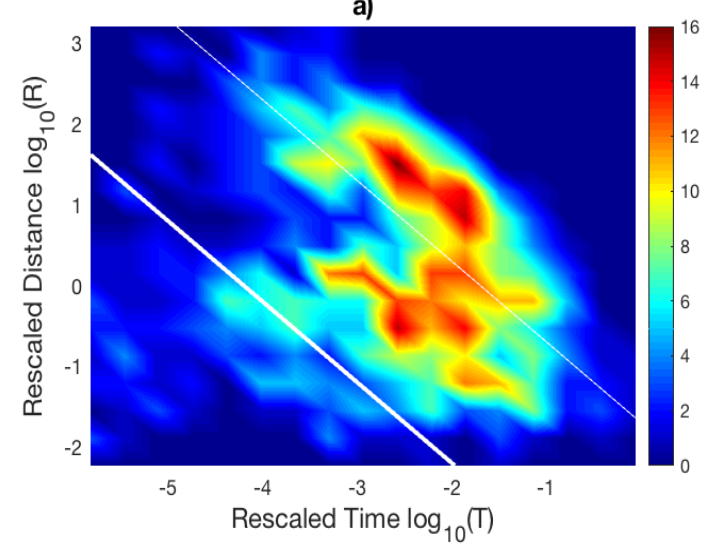

c)

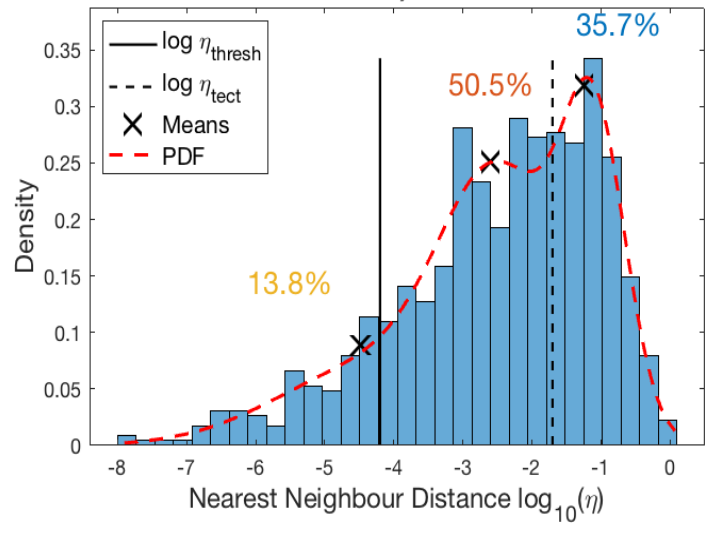

b)

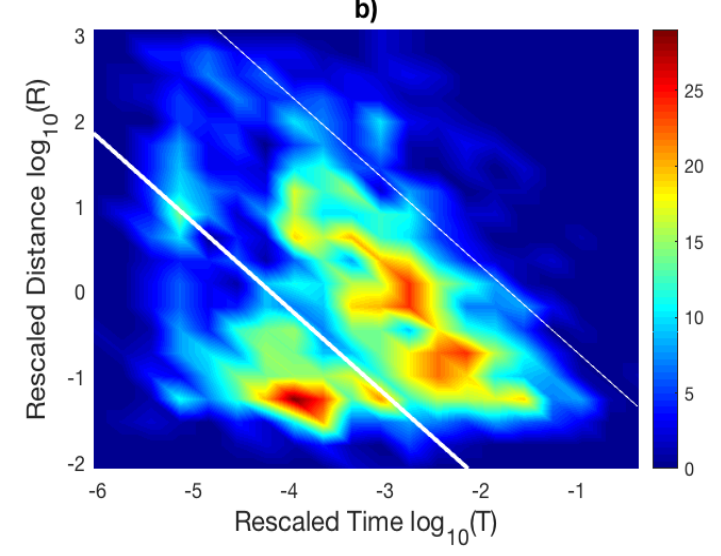

d)

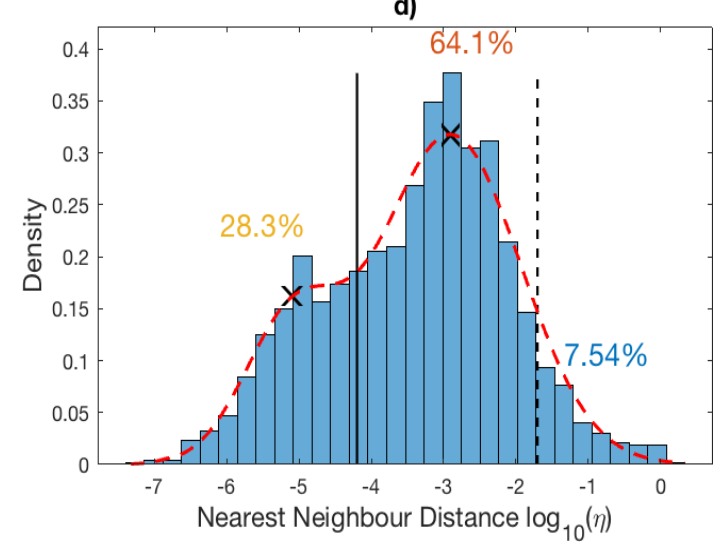

Figure B 4: $M_{0}=2.2$. (a, c) 1975-2009. (b, d) 2010-2018. (a, b) Joint distributions of the temporal and spatial components $(T, R)$. (c, d) Normalized densities of $\eta$ values. See Figure B 1 caption for a graphical description. 


$$
M_{0}=2.4
$$

a)

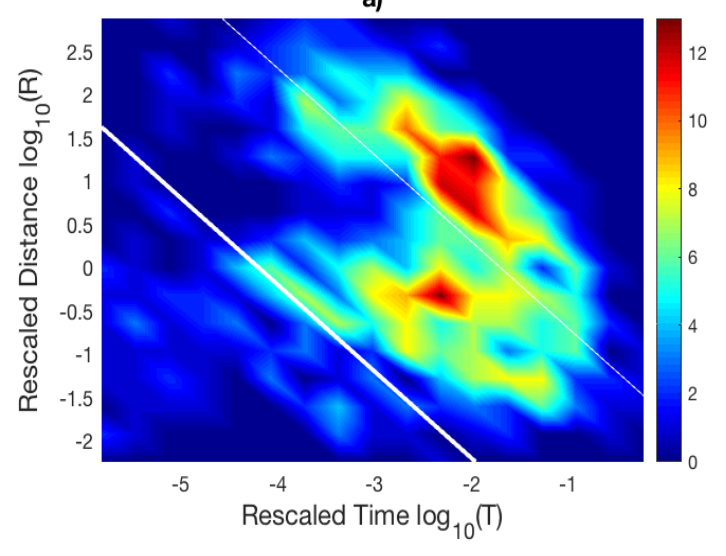

c)

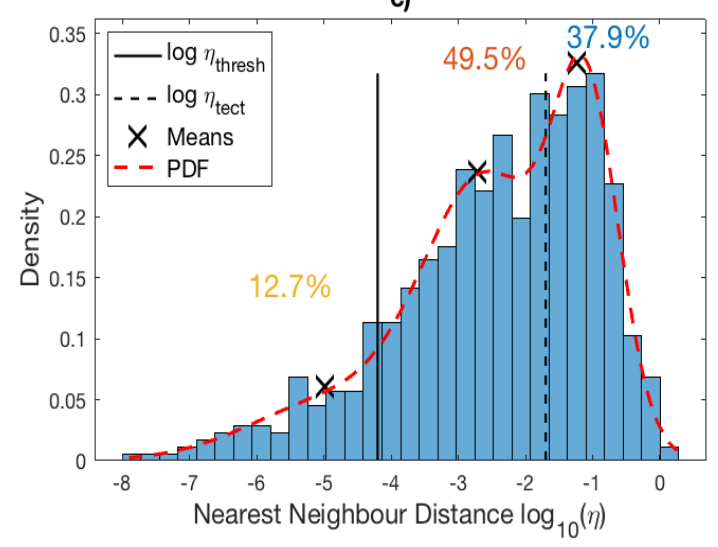

b)

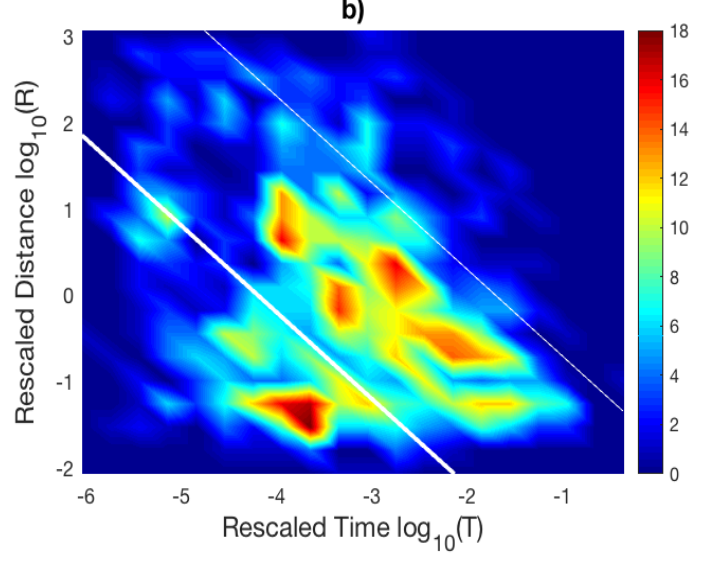

d)

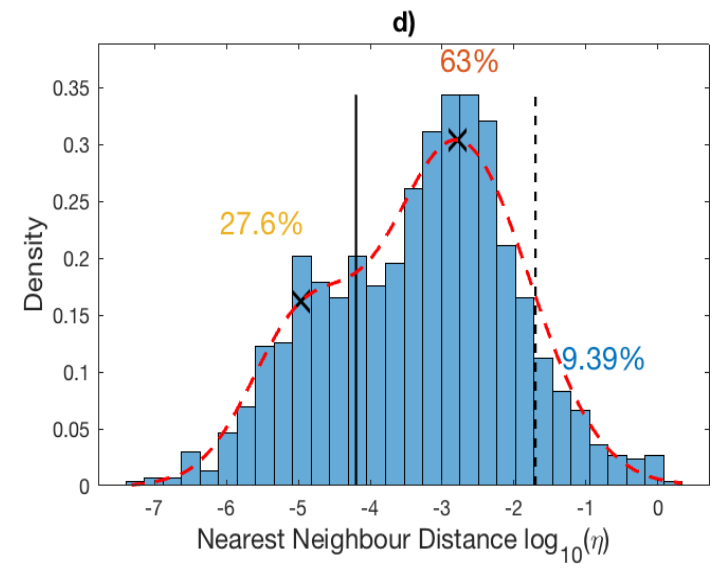

Figure B 5: $M_{0}=2.4$. (a, c) 1975-2009. (b, d) 2010-2018. (a, b) Joint distributions of the temporal and spatial components $(T, R)$. (c, d) Normalized densities of $\eta$ values. See Figure B 1 caption for a graphical description. 


$$
M_{0}=2.6
$$
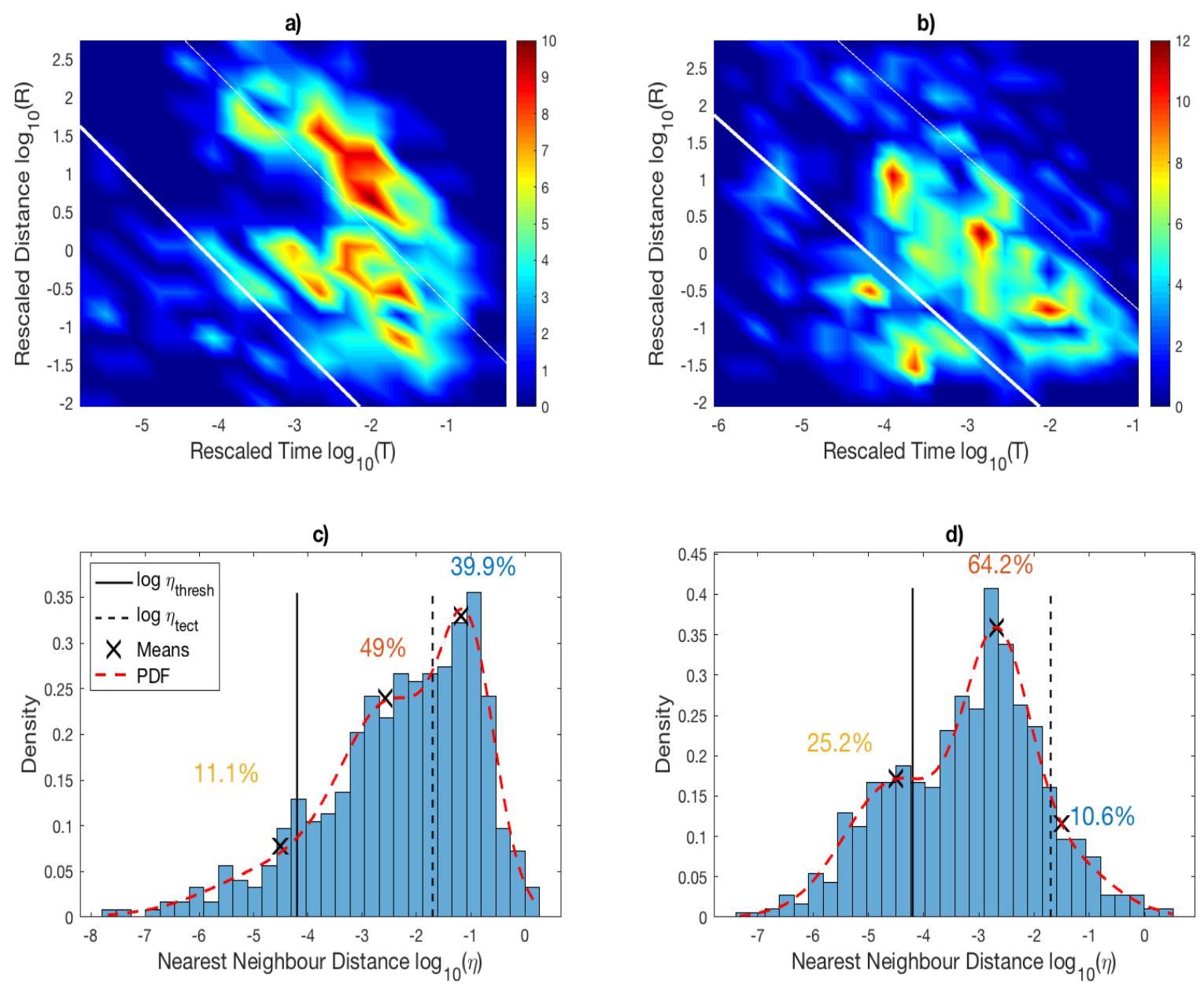

Figure B 6: $M_{0}=2$. 6. (a, c) 1975-2009. (b, d) 2010-2018. (a, b) Joint distributions of the temporal and spatial components $(T, R)$. (c, d) Normalized densities of $\eta$ values. See Figure B 1 caption for a graphical description. 


$$
M_{0}=2.8
$$
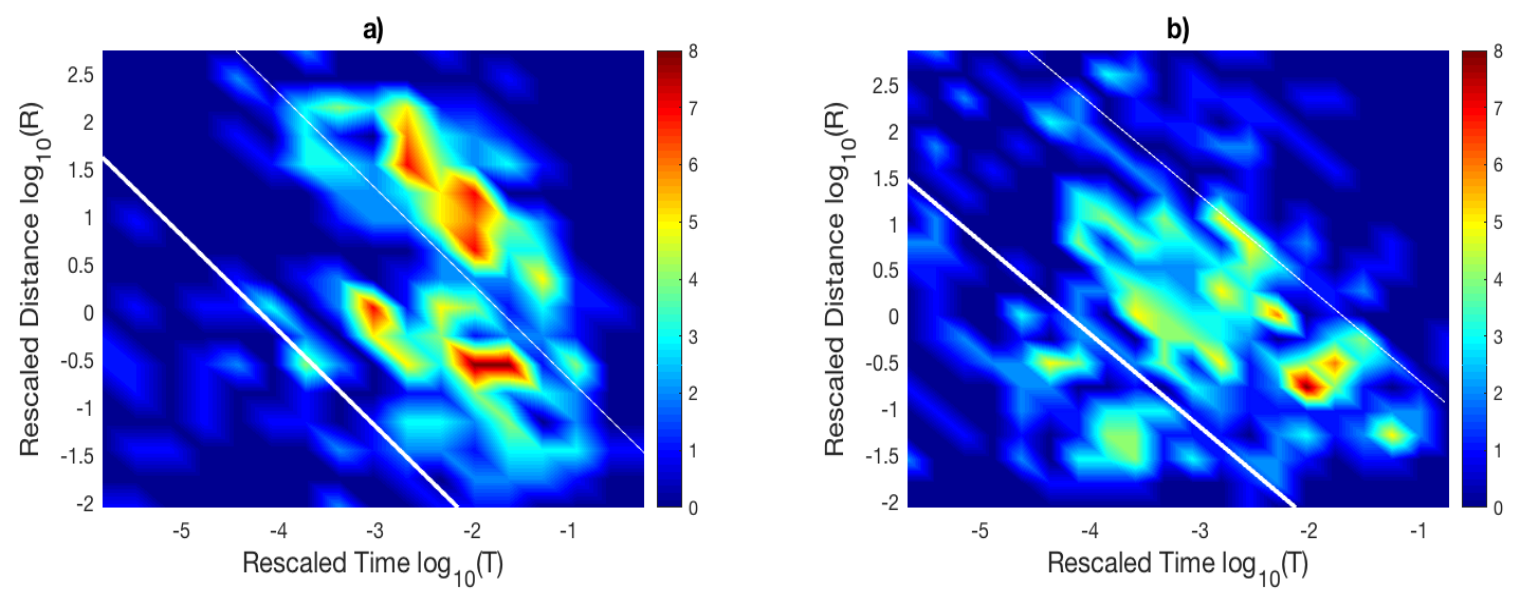

c)
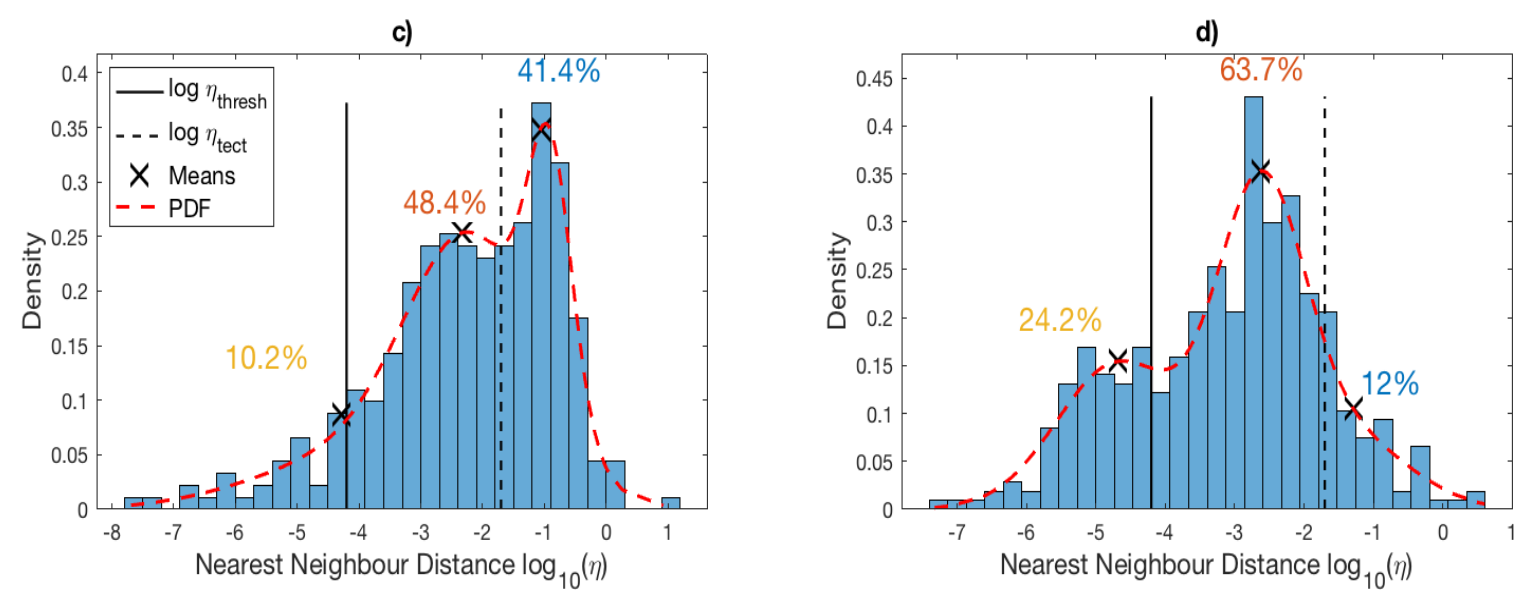

Figure B 7: $M_{0}=2.8$. (a, c) 1975-2009. (b, d) 2010-2018. (a, b) Joint distributions of the temporal and spatial components $(T, R)$. (c, d) Normalized densities of $\eta$ values. See Figure B 1 caption for a graphical description. 


$$
M_{0}=3.0
$$
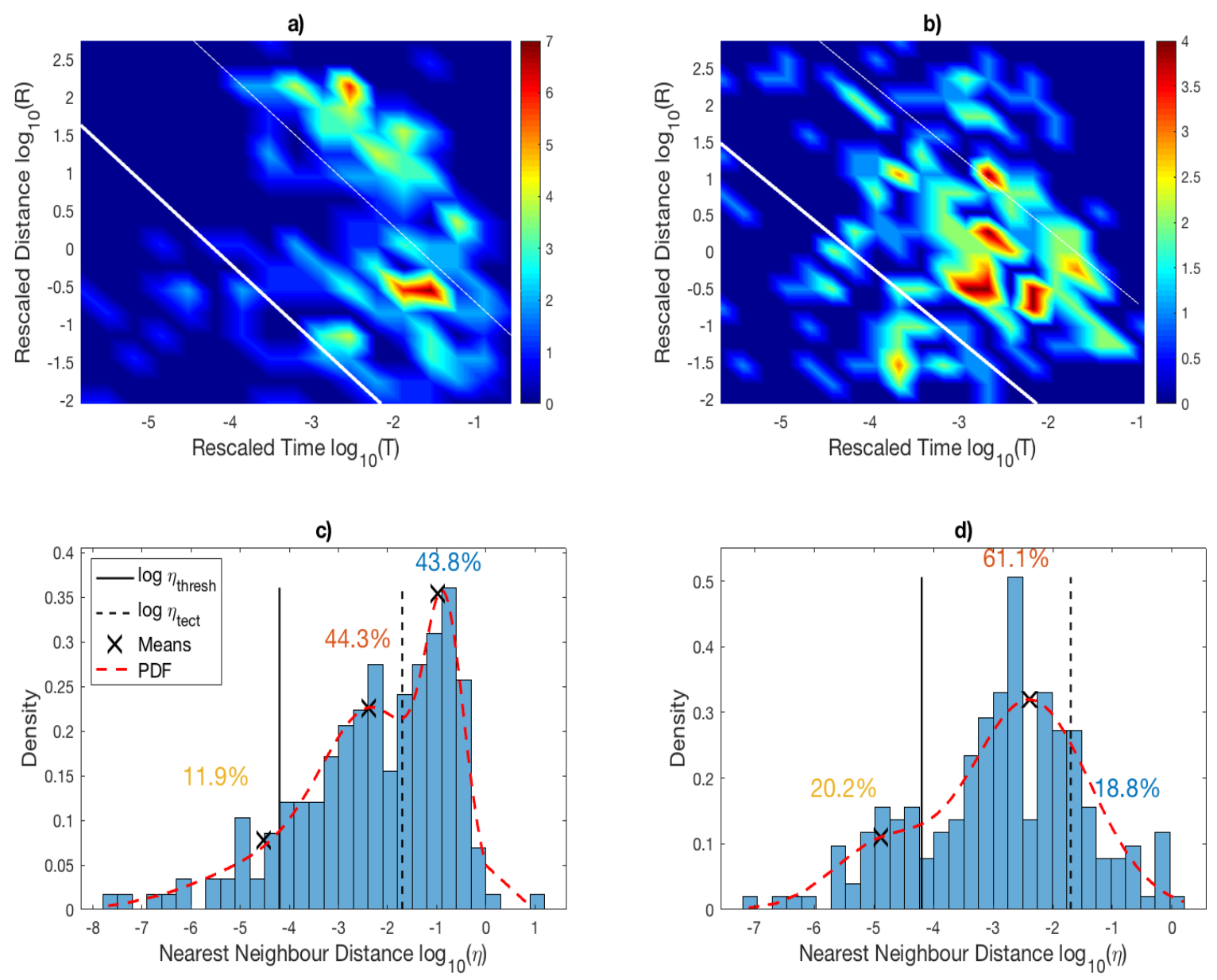

Figure B 8: $M_{0}=3.0$. (a, c) 1975-2009. (b, d) 2010-2018. (a, b) Joint distributions of the temporal and spatial components $(T, R)$. (c, d) Normalized densities of $\eta$ values. See Figure B 1 caption for a graphical description. 
Table B 1: Expectation Maximization results for the GMM applied to the WCSB regional catalogue for the first time period (1975-2009) using different cut-off magnitudes $M_{0} . k$ is the number of modes, AIC and BIC are the resulting Akaike and Bayes Information Criteria. Highlights show the minimized values.

\begin{tabular}{cccccccccc}
\hline $\boldsymbol{M}_{\mathbf{0}}$ & $\mathbf{0 . 5}$ & $\mathbf{1}$ & $\mathbf{2}$ & $\mathbf{2 . 2}$ & $\mathbf{2 . 4}$ & $\mathbf{2 . 6}$ & $\mathbf{2 . 8}$ & $\mathbf{3 . 0}$ \\
\hline $\mathbf{k}$ & & & \multicolumn{7}{c}{ AIC } \\
$\mathbf{1}$ & 6203.62 & 5986.04 & 3852.16 & 3061.67 & 2331.07 & 1654.25 & 1100.99 & 725.52 \\
$\mathbf{2}$ & 5893.12 & 5710.71 & 3709.98 & 2941.13 & 2237.45 & 1590.52 & 1067.61 & 697.33 \\
$\mathbf{3}$ & 5824.11 & 5638.43 & 3670.41 & 2907.56 & 2213.58 & 1579.34 & 1055.10 & 694.08 \\
\hline $\mathbf{4}$ & 5827.54 & 5640.95 & 3673.16 & 2911.06 & 2218.11 & 1584.39 & 1060.89 & 696.99 \\
\hline $\mathbf{k}$ & & & & & & & & & \\
\hline $\mathbf{1}$ & 6214.50 & 5996.86 & 3862.09 & 3071.14 & 2339.98 & 1662.51 & 1108.42 & 732.04 \\
\hline $\mathbf{2}$ & 5920.33 & 5737.74 & 3734.83 & 2964.81 & 2259.74 & 1611.18 & 1086.18 & 713.64 \\
\hline $\mathbf{3}$ & 5867.64 & 5681.68 & 3710.16 & 2945.45 & 2249.25 & 1612.39 & 1084.81 & 720.18 \\
\hline $\mathbf{4}$ & 5887.40 & 5700.42 & 3727.82 & 2963.15 & 2267.15 & 1629.83 & 1101.74 & 732.88 \\
\hline
\end{tabular}


Table B 2: Expectation Maximization results for the GMM applied to the WCSB regional catalogue for the second time period (2010-2018) using different cut-off magnitudes $M_{0} . k$ is the number of modes, AIC and BIC are the resulting Akaike and Bayes Information Criteria. Highlights show the minimized values.

\begin{tabular}{ccccccccccc}
\hline $\boldsymbol{M}_{\mathbf{0}}$ & $\mathbf{0 . 5}$ & $\mathbf{1}$ & $\mathbf{2}$ & $\mathbf{2 . 2}$ & $\mathbf{2 . 4}$ & $\mathbf{2 . 6}$ & $\mathbf{2 . 8}$ & $\mathbf{3 . 0}$ \\
\hline $\mathbf{k}$ & & & \multicolumn{7}{c}{ AIC } \\
$\mathbf{1}$ & 12438.04 & 12142.63 & 8355.68 & 6139.68 & 3988.65 & 2426.72 & 1414.20 & 727.27 \\
$\mathbf{2}$ & 12286.36 & 11996.86 & 8219.14 & 6040.12 & 3936.59 & 2407.57 & 1400.32 & 721.92 \\
$\mathbf{3}$ & 12292.60 & 12003.05 & 8223.26 & 6060.03 & 3951.39 & 2402.08 & 1397.78 & 723.25 \\
\hline $\mathbf{4}$ & 12296.09 & 12006.83 & 8224.55 & 6046.57 & 3941.60 & 2407.04 & 1403.60 & 723.47 \\
\hline $\mathbf{k}$ & & & & $\mathbf{B I C}$ & & & \\
\hline $\mathbf{1}$ & 12450.43 & 12154.98 & 8367.30 & 6150.71 & 3998.78 & 2435.84 & 1422.17 & 738.94 \\
\hline $\mathbf{2}$ & 12317.33 & 12027.73 & 8254.20 & 6087.68 & 3976.92 & 2430.36 & 1420.27 & 733.58 \\
\hline $\mathbf{3}$ & 12342.16 & 12052.44 & 8265.74 & 6084.13 & 3976.92 & 2438.55 & 1429.70 & 749.91 \\
\hline $\mathbf{4}$ & 12364.24 & 12074.75 & 8288.47 & 6107.20 & 3997.32 & 2457.18 & 1447.48 & 760.13 \\
\hline
\end{tabular}




\section{Curriculum Vitae}

Name:

Post-secondary

Education and Degrees:

Honours and Awards:

Related Work Experience
Sidhanth Kothari

University of Toronto

Toronto, Ontario, Canada

2010-2014 H. B.Sc

The University of Western Ontario

London, Ontario, Canada

2019 M.Sc

Geophysics Travel Scholarship, granted by the University of Western Ontario, 2015

Centre for Planetary Exploration Fellowship, granted by the University of Toronto, 2014

Teaching Assistant

The University of Western Ontario

2014-2016

Research Assistant

The University of Western Ontario

2014-2016

Research Assistant

University of Toronto

2013-2014 